Ann. Scient. Éc. Norm. Sup.,

$4^{\text {e }}$ série, t. 39, 2006, p. 75 à 175.

\title{
NAVIER-STOKES REGULARIZATION OF MULTIDIMENSIONAL EULER SHOCKS
}

\author{
By C.M.I. Olivier GUÈS ${ }^{1}$, Guy MÉTIVIER ${ }^{2}$, MARK WILliaMS ${ }^{3}$, \\ KEVIN ZUMBRUN ${ }^{4}$
}

\begin{abstract}
We establish existence and stability of multidimensional shock fronts in the vanishing viscosity limit for a general class of conservation laws with "real", or partially parabolic, viscosity including the Navier-Stokes equations of compressible gas dynamics with standard or van der Waals-type equation of state. More precisely, given a curved Lax shock solution $u^{0}$ of the corresponding inviscid equations for which (i) each of the associated planar shocks tangent to the shock front possesses a smooth viscous profile and (ii) each of these viscous profiles satisfies a uniform spectral stability condition expressed in terms of an Evans function, we construct nearby smooth viscous shock solutions $u^{\varepsilon}$ of the viscous equations converging to $u^{0}$ as viscosity $\varepsilon \rightarrow 0$, and establish for these sharp linearized stability estimates generalizing those of Majda in the inviscid case. Conditions (i)-(ii) hold always for shock waves of sufficiently small amplitude, but in general may fail for large amplitudes.

We treat the viscous shock problem considered here as a representative of a larger class of multidimensional boundary problems arising in the study of viscous fluids, characterized by sharp spectral conditions rather than symmetry hypotheses, which can be analyzed by Kreiss-type symmetrizers.

Compared to the strictly parabolic (artificial viscosity) case, the main new features of the analysis appear in the high frequency estimates for the linearized problem. In that regime we use frequency-dependent conjugators to decouple parabolic components that are smoothed from hyperbolic components (like density in Navier-Stokes) that are not. The construction of the conjugators and the subsequent estimates depend on a careful spectral analysis of the linearized operator.
\end{abstract}

(c) 2006 Elsevier SAS

RÉSUMÉ. - Nous démontrons l'existence et la stabilité d'ondes de chocs multidimensionnelles à viscosité évanescente pour une classe générale de systèmes de lois de conservation partiellement paraboliques possédant une viscosité «réaliste», et incluant le système des équations de Navier-Stokes de la dynamique des gaz compressibles pour une équation d'état standard ou de type Van der Vaals. Plus précisément, étant donnée une solution onde de choc $u^{0}$ du système hyperbolique sans viscosité pour laquelle (i) chaque choc plan tangent admet un profil de choc visqueux et (ii) chacun de ces profils de chocs satisfait une hypothèse spectrale de stabilité uniforme formulée en termes de fonction d'Evans, nous contruisons une famille de solutions $u^{\varepsilon}$ du problème visqueux qui convergent vers $u^{0}$ lorsque la viscosité $\varepsilon \rightarrow 0$. Nous établissons pour celles-ci des estimations linéarisées optimales qui généralisent les estimations obtenues par Majda dans le cas des ondes de choc sans viscosité. Les conditions (i) et (ii) sont toujours satisfaites pour des ondes de chocs d'amplitude suffisamment petite, mais peuvent ne pas l'être pour des chocs de grande amplitude.

\footnotetext{
${ }^{1}$ Partially supported by European network HYKE, HPRN-CT-2002-00282.

2 Partially supported by European network HYKE, HPRN-CT-2002-00282.

${ }^{3}$ Partially supported by NSF grants DMS-0070684 and DMS-0401252.

${ }^{4}$ Partially supported by NSF grants DMS-0070765 and DMS-0300487.
} 
Notre approche du problème de la stabilité des chocs visqueux est représentative d'une classe de problèmes aux limites multidimensionnels apparaissant en mécanique des fluides, qui sont caractérisés par des hypothèses de stabilité spectrales (plutôt que par des hypothèses de symétrie) et dont l'analyse s'effectue par des méthodes de «symétriseurs de Kreiss».

Par rapport au cas strictement parabolique (viscosité artificielle), les plus importantes nouveautés de l'analyse apparaissent dans les estimations à hautes fréquences pour le problème linéarisé. Dans ce régime, on introduit des matrices de conjugaison dépendant des variables de fréquence afin de découpler les composantes «paraboliques» qui sont régularisées, de celles «hyperboliques » qui ne le sont pas (comme la densité dans Navier-Stokes). La construction de ces matrices et l'obtention des estimations correspondantes nécessitent une analyse spectrale soignée de l'opérateur linéarisé.

(C) 2006 Elsevier SAS

\section{Introduction}

A number of equations in continuum mechanics may be written as viscous, or second-order perturbations

$$
\partial_{t} f_{0}\left(u^{\varepsilon}\right)+\sum_{j=1}^{d} \partial_{j} f_{j}\left(u^{\varepsilon}\right)-\varepsilon \sum_{j, k=1}^{d} \partial_{j}\left(B_{j, k}\left(u^{\varepsilon}\right) \partial_{k} u^{\varepsilon}\right)=0
$$

of first-order systems of hyperbolic conservation laws

$$
\partial_{t} f_{0}(u)+\sum_{j=1}^{d} \partial_{j} f_{j}(u)=0,
$$

where $u, f^{j} \in \mathbb{R}^{N}, B_{j, k} \in \mathbb{R}^{N \times N}$. Here the second-order $B_{j, k}$ terms model transport effects such as viscosity and heat, magnetic, or electric conduction, while the coefficient $\varepsilon$ is a dimensionless parameter depending on the length and time scales under consideration. The main examples that we have in mind are the Navier-Stokes and Euler equations of compressible gas dynamics, which have forms (1.1) and (1.2), respectively. In general, we will refer to $B_{j, k}$ terms as (generalized) viscosity terms, and $\frac{1}{\varepsilon}$ as a (generalized) Reynolds number. See Section 2 for a precise description of the Navier-Stokes and Euler equations.

Systems (1.2) are well known to support shock wave, or travelling discontinuity front solutions

$$
u_{0}(y, x, t)= \begin{cases}u_{0}^{-}, & x \leqslant \psi(t, y) \\ u_{0}^{+}, & x \geqslant \psi(t, y)\end{cases}
$$

Here $(t, y, x):=\left(x_{0}, x_{1}, \ldots, x_{d}\right)$ and $u_{0}^{ \pm}, \psi$ are smooth functions satisfying the RankineHugoniot jump conditions

$$
\sum_{j=0}^{d-1} \partial_{j} \psi\left[f_{j}(u)\right]=\left[f_{d}(u)\right]
$$

on the shock front $\{x=\psi(t, y)\}$, and (1.2) on the respective sides of the front. A wide class of such solutions has been constructed by A. Majda [26,27] under an appropriate spectral stability condition (the "uniform Lopatinski condition", definition (A.1)) on the family of all planar shock fronts tangent to $\psi$. A fundamental question in the theory of hyperbolic conservation laws, known as the "viscous profile" or "vanishing viscosity" problem, is whether there exist "viscous regularizations" of such inviscid shock solutions, that is, solutions $u_{\varepsilon}$ of the viscous

$4^{\text {e }}$ SÉRIE - TOME $39-2006-\mathrm{N}^{\circ} 1$ 
equations (1.1) such that $u_{\varepsilon}$ approaches $u_{0}$ in the vanishing viscosity limit $\varepsilon \rightarrow 0$. That is, does the behavior of solutions of the model (1.2) accurately predict the behavior of solutions of the more complicated model (1.1)?

In the case that (1.1) is strictly parabolic, a complete solution of the viscous profile problem was given in [12], extending partial results of [11], answering in the affirmative under the assumption of a viscous spectral stability condition (the "uniform Evans condition", definition (2.20)) analogous to that of Majda in the inviscid case. However, physical systems are typically not strictly parabolic, but rather of a composite, symmetric hyperbolic-partially parabolic form identified by Kawashima [21-23]; in particular, the Navier-Stokes equations of compressible gas- or magnetohydrodynamics (MHD) have this form. Thus, the problem remains open in the physical context from which it originally derived.

In this paper, we continue the program begun in [10-12], extending the results of [12] to a general class of hyperbolic-parabolic systems containing in particular the Navier-Stokes equations of compressible gas dynamics. Viscous regularizations are shown to exist assuming the uniform Evans condition; the condition is known to hold for weak shocks [34]. With suitable modifications, our methods can be applied also to the equations of MHD [14]. The class of systems we consider is somewhat more general than the one defined by Kawashima, and is defined by replacing his symmetrizability hypotheses with sharper spectral hypotheses. The relation between the two classes is analogous to that between the classes of hyperbolic and Friedrichs symmetric hyperbolic systems.

In the inviscid case the standard approach [26-28] toward the analysis of solutions (1.3) is to flatten the shock by the change of variables involving the unknown $\psi$

$$
\tilde{x}=x-\psi(t, y)
$$

transforming (1.2) into

$$
\sum_{j=0}^{d-1} A_{j}(u) \partial_{j} u+\widetilde{A}_{d}(u, d \psi) \partial_{\tilde{x}} u=0,
$$

where $A_{j}(u):=f_{j}^{\prime}(u)$ is the Jacobian matrix of $f_{j}$ and

$$
\widetilde{A}_{d}(u, d \psi):=A_{d}(u)-\sum_{j=0}^{d-1} \partial_{j} \psi A_{j}(u)
$$

is the boundary matrix. Eq. (1.6) is then solved separately on $\{\tilde{x}>0\}$ and $\{\tilde{x}<0\}$, together with the transmission conditions

$$
\sum_{j=0}^{d-1} \partial_{j} \psi\left[f_{j}(u)\right]=\left[f_{d}(u)\right] \quad \text { on }\{\tilde{x}=0\}
$$

induced by (1.4).

In the viscous case, under appropriate "physical" hypotheses (see Section 2, or discussion in [38, Section 1.3.1 and references therein]), the discontinuity is smoothed by the joint action of hyperbolic and parabolic terms, and so there is no well-defined front and no transmission condition (1.4). Nonetheless, following [12,17] we introduce an artificial unknown front $\psi^{\varepsilon}$ and perform the transformation

$$
\tilde{x}=x-\psi^{\varepsilon}(t, y)
$$


to convert the equations to a form

$$
\sum_{j=0}^{d-1} A_{j}\left(u^{\varepsilon}\right) \partial_{j} u^{\varepsilon}+\widetilde{A}_{d}\left(u^{\varepsilon}, d \psi^{\varepsilon}\right) \partial_{\tilde{x}} u^{\varepsilon}-\varepsilon \sum_{j, k=1}^{d} D_{j}\left(B_{j, k}\left(u^{\varepsilon}\right) D_{k} u^{\varepsilon}\right)=0
$$

analogous to the inviscid one, where $D_{j}=\partial_{j}-\left(\partial_{j} \psi^{\varepsilon}\right) \partial_{\tilde{x}}$ when $1 \leqslant j<d$ and $D_{d}=\partial_{\tilde{x}}$. Note that the function $\psi^{\varepsilon}$ which defines the "viscous front" is distinct from the function $\psi$ that defines the inviscid shock.

The introduction of the viscous front allows us to reformulate (1.10) as a transmission problem. Let $\widetilde{B}_{d, d}\left(u^{\varepsilon}, \nabla \psi^{\varepsilon}\right)$ be the coefficient of $\partial_{d}^{2}$ in (1.10). Since solutions are expected to be smooth in the viscous case, we set $u_{ \pm}^{\varepsilon}:=\left.u^{\varepsilon}\right|_{ \pm x \geqslant 0}$, replace the jump conditions (1.8) with tangency conditions

$$
\left[u^{\varepsilon}\right]=0, \quad\left[\widetilde{B}_{d, d}\left(u^{\varepsilon}, \nabla \psi^{\varepsilon}\right) \partial_{\tilde{x}} u^{\varepsilon}\right]=0 \quad \text { on } \tilde{x}=0,
$$

and observe that every smooth solution $u_{ \pm}^{\varepsilon}, \psi^{\varepsilon}$ of the transmission problem (1.10), (1.11) pieces together to give a smooth solution of (1.10), or equivalently (after changing back to the original variables), the original viscous problem (1.1) on the whole space.

This puts the viscous and inviscid problems into the common framework of fixed-boundary transmission problems and, in particular, allows us to apply Kreiss-type symmetrizers to the viscous problem. (Recall that in [19] Kreiss constructed symmetrizers for strictly hyperbolic boundary problems.) Still to be determined are the separation of viscous and inviscid effects in the enlarged system (1.10) and the relation of (1.11) to (1.8) in the singular limit $\varepsilon \rightarrow 0$. Note that the "size" of the viscous boundary-transmission system, and the number of transmission conditions both depend on the rank of $\widetilde{B}_{d, d}$; thus, (1.11) represents an appropriate generalization to real viscosity systems of the corresponding condition introduced in $[12,17]$ in the strictly parabolic case.

By suitable extension of the methods of [12,17], we find (see Section 5) that, provided (i) the inviscid solution $u_{0}$ (1.3) satisfies the spectral stability condition imposed by Majda on his constructed solutions and (ii) each tangent planar discontinuity has a transversal planar viscous profile (as defined in Section 2.3), then we may construct a hierarchy of approximate solutions

$$
u_{a}^{\varepsilon, M}=\sum_{0 \leqslant j \leqslant M} \varepsilon^{j} \mathcal{U}^{j}(t, y, \tilde{x}, \tilde{x} / \varepsilon), \quad \psi_{a}^{\varepsilon, M}=\sum_{0 \leqslant j \leqslant M} \varepsilon^{j} \psi^{j}(t, y)
$$

of (1.10)-(1.11) of order $M \geqslant 0$ satisfying the equations up to order $\varepsilon^{M+1}$. In this expansion the first term $\psi^{0}$ is the inviscid shock front (called $\psi$ in (1.3)) and

$$
\mathcal{U}^{0}(t, y, \tilde{x}, z)=u_{0}\left(t, y, \tilde{x}+\psi^{0}(t, y)\right)+V^{0}(t, y, z) \quad \text { in } \pm \tilde{x} \geqslant 0,
$$

where $V^{0}(t, y, z)$ decays exponentially to zero as $z \rightarrow \pm \infty$ and describes the viscous boundary layers on each side of the inviscid shock. In addition, for

$$
p(t, y)=\left(u_{0}^{+}\left(t, y, \psi^{0}(t, y)\right), u_{0}^{-}\left(t, y, \psi^{0}(t, y)\right), d \psi^{0}(t, y)\right),
$$

$W_{0}(z, p(t, y)):=\mathcal{U}^{0}(t, y, 0, z)$ satisfies the viscous profile equation (2.22), (2.23) associated to the inviscid shock.

The goal is then to convert the formal approximation error to a rigorous convergence error: that is, to show that $\left(u_{a}^{\varepsilon, M}, \psi_{a}^{\varepsilon, M}\right)$ is order $\varepsilon^{M+1}$ close to an exact solution $\left(u^{\varepsilon}, \psi^{\varepsilon}\right)$. The main issue therefore, as in $[11,12]$, is to establish sufficiently strong stability estimates about the

$4^{\text {e }}$ SÉRIE - TOME $39-2006-\mathrm{N}^{\circ} 1$ 
highly singular approximate solutions $u_{a}^{\varepsilon, M}, \psi_{a}^{\varepsilon, M}$ to carry out an appropriate nonlinear iteration for $u^{\varepsilon}, \psi^{\varepsilon}$.

To this end, we impose a spectral stability condition on the viscous shock profiles associated with the family of planar shocks tangent to the inviscid shock $\psi_{0}$, analogous to the condition imposed by Majda on the planar shocks themselves. As described in [36,39], this may be expressed in terms of an Evans function analogous to the Lopatinski determinant of the inviscid case; moreover, it is a striking fact that the viscous spectral condition in the low frequency regime is equivalent to the combination of the inviscid spectral condition and transversality of the viscous profile. The latter two conditions are the main ones needed for our construction of approximate solutions. A precise statement of the equivalence is given by Theorem A.2 in Appendix A. Thus, the viscous stability condition is a natural generalization of the uniform Lopatinski condition of Majda, which we call the uniform Evans condition. Like the Lopatinski condition, the uniform Evans condition is satisfied for sufficiently small-amplitude shocks [6,34], but may fail for large-amplitude shocks [5,37]. Under this condition we establish uniform stability estimates for $\varepsilon$ sufficiently small, estimates that reduce in the vanishing viscosity limit $\varepsilon \rightarrow 0$ to those established by Majda [26,27] in the inviscid case, and yield eventually the following main theorem. The hypotheses (H0)-(H10) are partitioned among Assumptions 2.1, 2.4, 2.6, 2.9, 2.21, and 4.1 .

THEOREM 1.1. - Given models (1.1)-(1.2) and a piecewise smooth inviscid shock solution $u_{0}, \psi_{0}$ of (1.6)-(1.8) defined on a time interval $0 \leqslant t \leqslant T$, satisfying hypotheses (H0)-(H9) and (H10)(a) (including the uniform Evans condition (H9)), there exist approximate solutions $u_{a}^{\varepsilon, M}$, $\psi_{a}^{\varepsilon, M}$ of (1.10)-(1.11) as described in (1.12) of all orders $M \geqslant 0$, and an exact solution $u^{\varepsilon}, \psi^{\varepsilon}$ of (1.10)-(1.11), such that, for all $2 \leqslant p \leqslant \infty$,

$$
\left\|u_{a}^{\varepsilon, M}-u^{\varepsilon}\right\|_{L^{p}([0, T], y, \tilde{x})} \leqslant C \varepsilon^{M+1}, \quad\left|\psi_{a}^{\varepsilon, M}-\psi^{\varepsilon}\right|_{L^{p}([0, T], y)} \leqslant C \varepsilon^{M+1} .
$$

Consequently,

$$
\left\|u^{\varepsilon}-u_{0}\right\|_{L^{p}([0, T], y, \tilde{x})} \leqslant C \varepsilon^{1 / p}, \quad\left|\psi^{\varepsilon}-\psi_{0}\right|_{L^{p}([0, T], y)} \leqslant C \varepsilon
$$

and therefore

$$
\left\|\tilde{u}_{\varepsilon}-\tilde{u}_{0}\right\|_{L^{p}([0, T], y, x)} \leqslant C \varepsilon^{1 / p}
$$

where $\tilde{u}_{\varepsilon}$ and $\tilde{u}_{0}$ denote the associated solutions of (1.1) and (1.2), and $C>0$ is a constant independent of $\varepsilon$.

This theorem is an immediate corollary of the more precise result Theorem 6.18. Together with our linearized stability estimates, Theorem 1.1 represents a natural extension of the results of Majda [26,27] for inviscid equations (1.2) to the partially parabolic viscous regularization (1.1), analogous to that carried out in [12] in the strictly parabolic case.

Our analysis in the low and medium frequency regimes follows that of [12], with appropriate modifications to accommodate partially parabolic viscosity. In particular, we use the central ideas introduced in [12] of working with the problem linearized with respect to both $u$ and $\psi$, and of introducing an extra boundary condition supplementing (1.11) in the form of a local front evolution rule. Having introduced the extra unknown $\psi^{\varepsilon}$ in (1.10), we should expect the problem to be underdetermined without some extra boundary condition. The key to the low frequency stability analysis, here as in [12], is to choose the extra boundary condition in a way that removes the translational degeneracy of the linearized problem in the low frequency regime. 
The high-frequency stability analysis of [12] in the strictly parabolic case was based on a relatively straightforward rescaling argument. Here it is trickier due to the partial parabolicity and is carried out in a different way. In this regime, after a careful spectral analysis of the full operator, we are able to use paradifferential conjugators to decouple parabolic components that are smoothed from hyperbolic components (like density in Navier-Stokes) that are not. The estimate of the hyperbolic components depends on a further spectral analysis of the corresponding block evaluated near the endstates of the profile, and on a choice of norms exponentially weighted in a suitable way along the profile. The estimate of the parabolic components proceeds just as in the case of artificial viscosity.

New arguments are also required in the construction of the approximate solutions, especially in the choice of boundary conditions for the "slow" parts of the higher order profiles. In addition, the weaker high frequency estimate associated with real viscosity requires, for its application to the small viscosity limit, a nonlinear iteration scheme quite different from the one used in [12].

The high frequency analysis and the associated identification of useful structural conditions on the equations sufficient to carry out the analysis represent the main contributions of the paper. In this paper and its successors we identify general classes of hyperbolic-parabolic systems characterized by sharp spectral hypotheses rather than symmetry hypotheses, which can be analyzed using Kreiss-type symmetrizers. Such problems include the viscous shock problem considered here, the Navier-Stokes noncharacteristic boundary layers studied in [13], and the viscous MHD shocks studied in [14].

Plan of the paper. In Section 2 we present the various assumptions made in our analysis and discuss the fully linearized problem. The assumptions are satisfied in particular for sufficiently small-amplitude, Lax-type shock waves of the Euler/Navier-Stokes equations of compressible flow, with standard or van der Waals-type equation of state. The main ideas and difficulties of the paper are already present in the frozen coefficient analysis of Section 3, which is completely free of paradifferential operators. Paradifferential operators are used only in Section 4 in order to extend the estimates of Section 3 to the variable coefficient case. This is essential in order to handle curved shocks. Section 4.2 contains all the needed paradifferential facts. In Section 5 we construct high order approximate solutions to the viscous transmission problem (1.10), (1.11), and in Section 6 we find exact solutions nearby. In Appendix A we give a proof the Zumbrun and Serre $[36,37,39]$ result, relating low frequency behavior of the Evans function to the Majda inviscid stability determinant.

Remark 1.2. - (1) We call attention to the convention on stating hypotheses described in Remark 2.25.

(2) We do not distinguish between $v^{\varepsilon}$ and $v_{\varepsilon}, u^{0}$ and $u_{0}, w^{ \pm}$and $w_{ \pm}$, etc. Especially when other indices are present, it is sometimes more convenient to have an index up rather than down or vice versa.

\section{Equations and assumptions}

\subsection{The physical equations}

Our assumptions are modeled on the fundamental example of the Navier-Stokes equations of compressible gas dynamics with general, possibly van der Waals type equation of state. The full Navier-Stokes equations are the $5 \times 5$ system

$4^{\text {e }}$ SÉRIE - TOME $39-2006-\mathrm{N}^{\circ} 1$ 


$$
\begin{aligned}
& \text { (a) } \rho_{t}+\sum_{j=1}^{3}(\rho v)_{x_{j}}^{j}=0 \\
& \text { (b) } \quad\left(\rho v^{i}\right)_{t}+\sum_{j=1}^{3}\left(\rho v^{i} v^{j}+p \delta_{i j}\right)_{x_{j}}=\sum_{j=1}^{3}\left\{\mu\left(v_{x_{j}}^{i}+v_{x_{i}}^{j}\right)+\lambda\left(\sum_{k=1}^{3} v_{x_{k}}^{k}\right) \delta_{i j}\right\}_{x_{j}} \\
& i=1,2,3 \\
& \text { (c) } \quad\left\{\rho\left(e+\frac{|v|^{2}}{2}\right)\right\}_{t}+\sum_{j=1}^{3}\left\{\rho v^{j}\left(e+\frac{|v|^{2}}{2}\right)+p v^{j}\right\}_{x_{j}} \\
& =\sum_{j=1}^{3}\left\{\mu \sum_{i=1}^{3} v^{i}\left(v_{x_{j}}^{i}+v_{x_{i}}^{j}\right)+\lambda u^{j} \sum_{k=1}^{3} v_{x_{k}}^{k}+\kappa \theta_{x_{j}}\right\}_{x_{j}}
\end{aligned}
$$

Here we choose to work with the unknowns $\rho, v=\left(v^{1}, v^{2}, v^{3}\right)$ and $\theta$ which denote the density, fluid velocity, and temperature, respectively. The pressure $p$ and internal energy $e$ are given smooth functions of $\rho>0$ and $\theta>0$ satisfying

$$
\partial_{\theta} e>0 .
$$

We take the viscosity coefficients $\mu, \lambda$ and the heat conduction coefficient $\kappa$ to satisfy

$$
\mu=\varepsilon \underline{\mu}(\rho, \theta), \quad \lambda=\varepsilon \underline{\lambda}(\rho, \theta), \quad \kappa=\varepsilon \underline{\kappa}(\rho, \theta)
$$

where $\mu, \underline{\lambda}$ and $\underline{\kappa}$ are positive functions. $\varepsilon>0$ is a small parameter that we will sometimes refer to as "the viscosity". Note that the condition (2.2), together with the condition $\kappa>0$, is necessary for the well posedness of the heat conduction equation in $\theta$. Note also that the inviscid system of Euler's equation (2.1) with $\varepsilon=0$ is hyperbolic only when

$$
\partial_{\rho} p>0
$$

which may hold on a strictly smaller domain of $(\rho, \theta)$ than does (2.2), as seen in the example of a van der Waals gas.

\subsection{Structural assumptions}

The Navier-Stokes system is a particular case of systems (1.1). We note that viscous terms appear only in the last four equations and that these terms involve second derivatives of $v$ and $\theta$ only. We split vectors $f \in \mathbb{R}^{5}$ into their first component $f^{1}$ and the remaining ones $f^{2} \in \mathbb{R}^{4}$. With $u^{1}=\rho$ and $u^{2}=(v, \theta)$ we see that the Navier-Stokes system has the structure of (1.1) with

$$
A_{0}(u):=f_{0}^{\prime}(u)=\left(\begin{array}{cc}
A_{0}^{11} & 0 \\
A_{0}^{21} & A_{0}^{22}
\end{array}\right), \quad B_{j k}(u)=\left(\begin{array}{cc}
0 & 0 \\
0 & B_{j k}^{22}
\end{array}\right),
$$

where $M^{\alpha \beta}$ denote the sub-blocks of the matrix $M$ corresponding to the splitting $u=\left(u^{1}, u^{2}\right)$. In particular,

$$
\bar{B}_{j, k}(u):=A_{0}(u)^{-1} B_{j k}(u)=\left(\begin{array}{cc}
0 & 0 \\
0 & \bar{B}_{j k}^{22}(u)
\end{array}\right) .
$$

Extending these properties we consider systems (1.1) which satisfy (2.5): 
ASSUMPTION 2.1.-

(H0) (Smooth fluxes and viscosity.) Let $\mathcal{U}^{*}$ be an open subset of $\mathbb{R}^{N}$. The fluxes $f_{j}$ are $C^{\infty}$ functions of $u \in \mathcal{U}^{*}$ with values in $\mathbb{R}^{N}$ and the $B_{j, k}$ are $C^{\infty} N \times N$ matrices on $\mathcal{U}^{*}$. Moreover, for all $u \in \mathcal{U}^{*}$, the matrix $f_{0}^{\prime}(u)$ is invertible.

(H1) (Block form.) Possibly after a change of variables $u$ and multiplication on the left by an invertible constant coefficient matrix, there is $s \in\{1, \ldots, N\}$ and there are coordinates $u=\left(u^{1}, u^{2}\right) \in \mathbb{R}^{N-s} \times \mathbb{R}^{s}$ and $f=\left(f^{1}, f^{2}\right) \in \mathbb{R}^{N-s} \times \mathbb{R}^{s}$ such that the block form condition (2.5) is satisfied.

Remark 2.2. - This assumption can be put in a more geometric form, at least locally. First observe that under a change of variables $u=\Phi(\tilde{u})$, the fluxes $f_{j}$ and matrices $B_{j, k}$ are changed to

$$
\tilde{f}_{j}=f_{j} \circ \Phi, \quad \widetilde{B}_{j, k}=\left(B_{j, k} \circ \Phi\right) \Phi^{\prime}
$$

so that $\bar{B}_{j, k}$ is changed to

$$
\widetilde{A}_{0}^{-1} \widetilde{B}_{j, k}=\left(\Phi^{\prime}\right)^{-1}\left(\bar{B}_{j, k} \circ \Phi\right) \Phi^{\prime} .
$$

When (2.6) holds, the kernel and image of $\bar{B}_{j, k}=A_{0}^{-1} B_{j, k}$ satisfy

$$
\operatorname{ker} \bar{B}_{j, k}(u) \supset \mathbb{K}:=\mathbb{R}^{N-s} \times\{0\}^{s}, \quad \operatorname{Im} \bar{B}_{j, k}(u) \subset \mathbb{I}:=\{0\}^{N-s} \times \mathbb{R}^{s} .
$$

Moreover,

$$
\operatorname{Im} B_{j, k}(u) \subset \mathbb{J}:=\{0\}^{N-s} \times \mathbb{R}^{s} .
$$

Conversely, locally, Assumption 2.1 is the conjunction of five properties, which can be checked in any coordinate system $u$ :

(1) there is a space $\mathbb{K}(u)$ of dimension $N-s$ such that for all $u \in \mathcal{U}^{*}, \operatorname{ker} \bar{B}_{j, k}(u) \supset \mathbb{K}(u)$;

(2) there is a space $\mathbb{J}$ of dimension $s$ such that for all $u \in \mathcal{U}^{*}, \operatorname{Im} B_{j, k}(u) \subset \mathbb{J}$;

(3) for all $u \in \mathcal{U}^{*}, \mathbb{R}^{N}=\mathbb{K}(u) \oplus \mathbb{I}(u)$, where $\mathbb{I}(u)=A_{0}(u)^{-1} \mathbb{J}$;

(4) the vector bundle $\mathbb{K}(u)$ is smooth and integrable;

(5) the vector bundle $\mathbb{I}(u)$ is smooth and integrable.

Indeed, (3)-(5) imply that, at least locally, there is a change of coordinates $u=\Phi(\tilde{u})$, with $\tilde{u}=\left(u^{1}, u^{2}\right) \in \mathbb{R}^{N-s} \times \mathbb{R}^{s}$ such that

$$
\mathbb{K}(u)=\Phi^{\prime}(\tilde{u})\left(\mathbb{R}^{N-s} \times\{0\}^{s}\right), \quad \mathbb{I}(u)=\Phi^{\prime}(\tilde{u})\left(\{0\}^{N-s} \times \mathbb{R}^{s}\right) .
$$

By (2), we can choose linear coordinates $f=\left(f^{1}, f^{2}\right)$ such that $\mathbb{J}=\left\{f^{1}=0\right\}=\{0\}^{N-s} \times \mathbb{R}^{s}$. This implies that $\widetilde{B}_{j, k}=\left(B_{j, k} \circ \Phi\right) \Phi^{\prime}$ has the block diagonal form in (2.5). Moreover, $\widetilde{A}_{0}^{-1}=$ $\left(\Phi^{\prime}\right)^{-1}\left(A_{0}^{-1} \circ \Phi\right)$ maps $\mathbb{J}$ to $\mathbb{J}$, thus $\widetilde{A}_{0}^{-1}$ and $\widetilde{A}_{0}$ have the triangular form in (2.5). This shows that the conditions in (H1) are satisfied in the coordinates $\tilde{u}$.

Example 2.3. - We have already shown that $(\mathrm{H} 1)$ is satisfied for Navier-Stokes equations. More generally, suppose that there are splittings of coordinates $u=\left(u^{1}, u^{2}\right) \in \mathbb{R}^{N-s} \times \mathbb{R}^{s}$, $f=\left(f^{1}, f^{2}\right) \in \mathbb{R}^{N-s} \times \mathbb{R}^{s}$ such that

$$
f_{0}^{1}(u)=\hat{f}_{0}^{1}\left(u_{1}\right), \quad B_{j, k}(u) \partial_{k} u=\left(\begin{array}{c}
0 \\
\widehat{B}_{j, k}(u) \partial_{k}(\Psi(u))
\end{array}\right),
$$

where $\Psi \in C^{\infty}\left(\mathcal{U}^{*} ; \mathbb{R}^{s}\right)$ and the $\widehat{B}_{j, k}$ are $s \times s$ smooth matrices. Then, if the mapping $u \mapsto\left(u^{1}, \psi(u)\right)$ is a diffeomorphism, taking $u^{1}$ and $\psi(u)$ as coordinates, we see that (H1) is satisfied.

$4^{e}$ SÉRIE - TOME $39-2006-\mathrm{N}^{\circ} 1$ 
The assumption (H1) has an important consequence for the structure of Eq. (1.1) written in nonconservative form: it reads

$$
\sum_{j=0}^{d} A_{j}(u) \partial_{j} u-\varepsilon \sum_{j, k=1}^{d} B_{j, k}(u) \partial_{j, k}^{2} u-\varepsilon \sum_{j, k=1}^{d} g_{j, k}\left(u, \partial_{j} u, \partial_{k} u\right)=0
$$

with

$$
g_{j, k}(u, v, w)=\left(v \cdot \nabla_{u} B_{j, k}(u)\right) w
$$

bilinear in $v$ and $w$. Moreover, (2.5) implies that the first $N-s$ components of $g_{j, k}$ vanish:

$$
g_{j, k}(u, v, w)=\left(\begin{array}{c}
0 \\
g_{j, k}^{2}(u, v, w)
\end{array}\right)
$$

This is useful in the nonlinear stability analysis, since it allows us to consider terms like $\varepsilon g$ as lower order perturbations.

The triangular form of the equations also reveals the importance of the $(1,1)$ block:

$$
\begin{aligned}
& L^{11}(u, \partial)=\sum_{j=0}^{d} A_{j}^{11}(u) \partial_{j}, \quad \text { or } \\
& \bar{L}^{11}(u, \partial)=\left(A_{0}^{11}(u)\right)^{-1} L^{11}(u, \partial),
\end{aligned}
$$

which plays a special role in the analysis.

From now on we work with variables $u=\left(u^{1}, u^{2}\right) \in \mathcal{U}^{*}$ such that (2.5) and (2.6) hold. We set

$$
A_{j}=f_{j}^{\prime}, \quad \bar{A}_{j}=A_{0}^{-1} A_{j}, \quad \bar{B}_{j, k}=A_{0}^{-1} B_{j, k},
$$

and systematically use the notation $M^{\alpha \beta}$ for the sub-blocks of a matrix $M$ corresponding to the splitting $u=\left(u^{1}, u^{2}\right)$.

ASSUMPTION 2.4.-

(H2) (Partial parabolicity.) There is $c>0$ such that for all $u \in \mathcal{U}^{*}$ and $\xi \in \mathbb{R}^{d}$, the eigenvalues of $\bar{B}^{22}(u, \xi)=\sum_{j, k=1}^{d} \xi_{j} \xi_{k} \bar{B}_{j, k}^{2,2}(u)$ satisfy $\operatorname{Re} \mu \geqslant c|\xi|^{2}$.

(H3) (Hyperbolicity of $(1,1)$ block.) The eigenvalues of $\bar{A}^{11}(u, \xi)=\sum_{j=1}^{d} \xi_{j} \bar{A}_{j}^{11}(u)$ are real and semisimple with constant multiplicities for $u \in \mathcal{U}^{*}$ and $\xi \in \mathbb{R}^{d} \backslash\{0\}$.

Remark 2.5. - (1) Assuming (H1), the condition (H2) is equivalent to the following coordinate-independent condition:

$\left(\mathrm{H} 2^{\prime}\right)$ for all $u \in \mathcal{U}^{*}$ and $\xi \in \mathbb{R}^{d} \backslash\{0\}, 0$ is an eigenvalue of $\bar{B}(u, \xi)=\sum \xi_{j} \xi_{k} \bar{B}_{j, k}(u)$ with constant multiplicity $N-s$ and the remaining eigenvalues satisfy $\operatorname{Re} \mu \geqslant c|\xi|^{2}$.

(2) When $s=N-1$, which is the case for the Navier-Stokes equations, then

$$
\bar{A}^{11}(u, \xi)=\sum_{j=1}^{d} \xi_{j} \bar{A}_{j}^{11}(u)
$$


with $\bar{A}_{j}^{11}(u) \in \mathbb{R}$. Therefore, the condition (H3) is automatically satisfied. (H3) is also clearly satisfied in cases where $\bar{A}^{11}(u, \xi)$ is a (real) scalar multiple of the identity $I_{N-s}$, as occurs for MHD in the case of infinite magnetic permeability.

Next we assume that the inviscid equations are hyperbolic for $u$ in some open subdomain $\mathcal{U} \subset \mathcal{U}^{*}$. Let

$$
\bar{A}(u, \xi)=\sum_{j=1}^{d} \xi_{j} \bar{A}_{j}(u) \quad \text { and } \quad \bar{B}(u, \xi)=\sum_{j, k=1}^{d} \xi_{j} \xi_{k} \bar{B}_{j, k}(u) .
$$

ASSUMPTION 2.6.-

(H4) (Hyperbolicity near endstates.) For $u \in \mathcal{U}$ and $\xi \in \mathbb{R}^{d} \backslash\{0\}$ the eigenvalues of $\bar{A}(u, \xi)$ are real and semisimple with constant multiplicity.

(H5) (Strict dissipativity near endstates.) There is $c>0$ such that for $u \in \mathcal{U}$ and $\xi \in \mathbb{R}^{d}$, the eigenvalues $\mu$ of $i \bar{A}(u, \xi)+\bar{B}(u, \xi)$ satisfy

$$
\Re \mu \geqslant \frac{c|\xi|^{2}}{1+|\xi|^{2}} .
$$

Remark 2.7. - (1) It is important for applications to allow that $\mathcal{U}$ can be strictly smaller than $\mathcal{U}^{*}$. For instance, for Euler's equation $\mathcal{U}$ is the sub-domain of states such that $\partial_{\rho} p>0$; recall Section 2.1. Note also that for such states $u$, Euler's equation satisfies the constant multiplicity assumption (H4).

The hypothesis (H4), which plays a role only in our low frequency analysis, is violated by the equations of viscous MHD, where characteristics of variable multiplicity appear in the hyperbolic part. Symmetrizers for viscous MHD in the low frequency regime are constructed in [14]. MHD can be treated by combining the low frequency analysis of [14] with the medium and high frequency analysis given here.

(2) Hypothesis (H2) is clearly satisfied by the Navier-Stokes equations when $\partial_{\theta} e>0$. We refer to [22,23] or [38, Remark 1.25], for verification that the Navier-Stokes equations satisfy (H5) whenever $\partial_{\theta} e>0, \partial_{\rho} p>0$. More generally, for systems that are symmetrizable in the sense that the matrices $A_{j}, B_{j, k}$ may be taken symmetric, (2.16) is equivalent to the genuine coupling condition of Kawashima: no eigenvector of $\bar{A}(u, \xi)$ lies in the kernel of $\bar{B}(u, \xi)$ for $\xi \in \mathbb{R}^{d} \backslash\{0\}$. This condition is checked for the Navier-Stokes equations in [23].

(3) There is a slight redundancy in Assumption 2.6, since (H5) implies that the eigenvalues of $\bar{A}(u, \xi)$ are real.

\subsection{Profiles and Evans functions}

Next we consider planar shocks. Denoting by $\left(y_{1}, \ldots, y_{d-1}, x\right)$ the space variables, we consider solutions of the inviscid equation (1.2) consisting of two constant states $u^{-}$and $u^{+}$ in $\mathcal{U}$ separated by a plane $\left\{x=h_{0} t+\sum_{j=1}^{d-1} h_{j} y_{j}\right\}$. The states $u^{ \pm}$and the front $h$ must satisfy the Rankine-Hugoniot condition:

$$
\sum_{j=0}^{d-1} h_{j}\left[f_{j}(u)\right]=\left[f_{d}(u)\right]
$$

where $[f]$ denotes $f\left(u^{+}\right)-f\left(u^{-}\right)$. With $\nu=\nu(h):=\left(-h_{0},-h_{1}, \ldots,-h_{d-1}, 1\right)$, let us introduce the normal flux and the normal boundary matrices

$4^{e}$ SÉRIE - TOME $39-2006-\mathrm{N}^{\circ} 1$ 


$$
\begin{aligned}
& \tilde{f}_{d}(u, \nu)=\sum_{j=0}^{d} \nu_{j} f_{j}(u), \\
& \widetilde{A}_{d}(u, \nu)=\sum_{j=0}^{d} \nu_{j} A_{j}(u), \quad \bar{A}_{d}(u, \nu)=A_{0}(u)^{-1} \widetilde{A}_{d}(u, \nu), \\
& \widetilde{B}_{d, d}(u, \nu)=\sum_{j, k=1}^{d} \nu_{j} \nu_{k} B_{j, k}(u) .
\end{aligned}
$$

Remark 2.8. - Let us also define for $j=1, \ldots, d-1$

$$
\widetilde{B}_{j, d}(u, \nu)=\sum_{k=1}^{d} \nu_{k} B_{j, k}(u), \quad \widetilde{B}_{d, j}(u, \nu)=\sum_{k=1}^{d} \nu_{k} B_{k, j}(u) .
$$

Observe that Eq. (1.10) can then be rewritten

$$
\sum_{j=0}^{d-1} A_{j}(u) \partial_{j} u+\widetilde{A}_{d}(u, \nu(d \psi)) \partial_{d} u-\varepsilon \sum_{j, k=1}^{d} \partial_{j}\left(\widetilde{B}_{j, k}(u, \nu(d \psi)) \partial_{k} u\right)=0 .
$$

We will sometimes abuse notation slightly and write, for example, $\widetilde{A}_{d}(u, d \psi)$ in place of $\widetilde{A}_{d}(u, \nu(d \psi))$.

In the viscous case, discontinuities are replaced by profiles, and shocks are replaced by travelling waves

$$
u^{\varepsilon}(t, y, x)=w\left(\frac{x-\psi(t, y)}{\varepsilon}\right), \quad \psi(t, y):=h_{0} t+\sum_{j=1}^{d-1} h_{j} y_{j} .
$$

Then $u^{\varepsilon}$ is a solution of (1.1) if and only if $w$ solves the profile equation

$$
\partial_{z}\left(\tilde{f}_{d}(w(z), \nu)\right)-\partial_{z}\left(\widetilde{B}_{d, d}(w(z), \nu) \partial_{z} w\right)=0 .
$$

The profile is associated to a shock $p=\left(u^{-}, u^{+}, h\right)$ when

$$
\lim _{z \rightarrow-\infty} w(z)=u^{-} \quad \text { and } \quad \lim _{z \rightarrow+\infty} w(z)=u^{+} .
$$

Recall that the Rankine-Hugoniot conditions (2.17) follow from (2.22), (2.23).

The next two assumptions mean that we are considering a family of profiles associated to Lax shocks, with an additional assumption of "hyperbolicity in the $\nu$ direction" for the $(1,1)$ block.

ASSUMPTION 2.9.-

(H6) (Lax shocks.) We are given a $C^{\infty}$ manifold $\mathcal{C} \subset \mathcal{U} \times \mathcal{U} \times \mathbb{R}^{d}$, with $\overline{\mathcal{C}}$ compact, such that each $p=\left(u^{-}, u^{+}, h\right)$ in $\mathcal{C}$ satisfies $(2.17)$. Moreover, the boundary matrices $\bar{A}_{d}\left(u^{ \pm}, \nu(h)\right)$ are invertible and the numbers $N_{-}$(respectively $N_{+}$) of positive (respectively negative) eigenvalues of $\bar{A}_{d}\left(u^{-}, \nu\right)$ (respectively $\bar{A}_{d}\left(u^{+}, \nu\right)$ ) satisfy $N_{+}+$ $N_{-}=N+1$.

(H7) (Shock profiles.) We are given a $C^{\infty}$ function $W_{0}(z, p)$ from $\mathbb{R} \times \mathcal{C}$ to $\mathcal{U}^{*}$ such that for all $p \in \mathcal{C}, W_{0}(\cdot, p)$ is a solution of (2.22), (2.23). We refer to such a function as a "shock profile". 
(H8) (Hyperbolicity of $(1,1)$ block with respect to $\nu$.) For each $p=\left(u^{+}, u^{-}, h\right) \in \mathcal{C}$, one of $\pm \bar{A}_{d}^{11}(w, \nu(h))$ has only strictly positive eigenvalues for all $w$ in the closed orbit $\left\{W_{0}(z, p): z \in \mathbb{R}\right\}^{c l}$.

In addition to the parameters $p=\left(u^{-}, u^{+}, h\right)$, we introduce new parameters $\left(u^{\prime}, h^{\prime}\right) \in \mathbb{R}^{N} \times$ $\mathbb{R}^{d}$ to represent perturbations of profiles and fronts. We set

$$
q=\left(p, u^{\prime}, h^{\prime}\right), \quad W(z, q)=W_{0}(z, p)+u^{\prime}, \quad W( \pm \infty, q)=u^{ \pm}+u^{\prime}
$$

and

$$
\begin{aligned}
& A_{j}(z, q):=A_{j}(W(z, q)), \quad \bar{A}_{j}(z, q)=\bar{A}_{j}(W(z, q)), \quad j \leqslant d-1, \\
& \widetilde{A}_{d}(z, q)=\widetilde{A}_{d}\left(W(z, q), \nu\left(h+h^{\prime}\right)\right), \quad \bar{A}_{d}(z, q)=\bar{A}_{d}\left(W(z, q), \nu\left(h+h^{\prime}\right)\right) .
\end{aligned}
$$

Proposition 2.10. - Let $W(z, q)$ be as in (2.24) and assume (H3) and (H8). There exists a neighborhood $\mathcal{O}$ of $(0,0) \in \mathbb{R}_{u^{\prime}}^{N} \times \mathbb{R}_{h^{\prime}}^{d}$ such that for all $z \in \mathbb{R} \cup\{ \pm \infty\}, p \in \mathcal{C}$, and $\left(u^{\prime}, h^{\prime}\right) \in \mathcal{O}$, we have $W(z, q) \in \mathcal{U}^{*}$ and

(a) one of $\pm \bar{A}_{d}^{11}(z, q)$ has only strictly positive eigenvalues;

(b) the eigenvalues of $\widetilde{A}_{d}^{11}(z, q)^{-1}\left(A_{0}^{11}(z, q) \tau+\sum_{j=1}^{d-1} A_{j}^{11}(z, q) \eta_{j}\right)$

are real and semisimple with constant multiplicity for $(\tau, \eta) \in \mathbb{R}^{d} \backslash 0$. In addition there exists $Z>0$ such that for all $q$ as above,

$$
|z| \geqslant Z \Longrightarrow W(z, q) \in \mathcal{U} .
$$

Proof. - (1) For $\xi \in \mathbb{C}^{d+1}$ the homogeneous polynomial $\pi(\xi)$ is said to be hyperbolic in the real direction $\beta$ if and only if $\pi(\beta) \neq 0$ and for all real $\xi^{\prime} \notin \mathbb{R} \beta$ the roots $r \in \mathbb{C}$ of $\pi\left(r \beta+\xi^{\prime}\right)$ are real. For $(z, q)$ as above, (H3) implies that

$$
\pi(z, q, \xi)=\operatorname{det}\left(\sum_{j=0}^{d} A_{j}^{11}(z, q) \xi_{j}\right)
$$

is hyperbolic in the direction $d t=(1,0, \ldots, 0)$. (2.26)(a) holds if and only if one of $\pm \nu\left(h+h^{\prime}\right)$ lies in the same component as $d t$ of the open set $\{\xi: \pi(z, q, \xi) \neq 0\}$ [18, Lemma 8.7.3]. Hypothesis (H8), compactness of $\overline{\mathcal{C}}$, and compactness of the closed orbit associated to each $p \in \mathcal{C}$ allow us to choose $\mathcal{O}$ so this is the case. $\pi(z, q, \xi)$ is hyperbolic with respect to all directions in that component, so (2.26)(a) implies that the roots $r$ of

$$
\pi\left(z, q, r \nu\left(h+h^{\prime}\right)+\xi^{\prime}\right)=0, \quad \xi^{\prime} \notin \mathbb{R} \nu,
$$

are real, which is equivalent to saying the eigenvalues in (2.26)(b) are real.

(2) Changing the notation for frequencies, we set

$$
\begin{aligned}
\mathcal{A}^{11}\left(z, q, \eta, \xi_{d}\right) & =\sum_{j=1}^{d-1} \bar{A}_{j}^{11}(z, q) \eta_{j}+\bar{A}_{d}^{11}(z, q) \xi_{d} \\
\mathcal{G}^{11}(z, q, \tau, \eta) & =\widetilde{A}_{d}^{11}(z, q)^{-1}\left(A_{0}^{11}(z, q) \tau+\sum_{j=1}^{d-1} A_{j}^{11}(z, q) \eta_{j}\right) .
\end{aligned}
$$

$4^{e}$ SÉRIE - TOME $39-2006-\mathrm{N}^{\circ} 1$ 
(H3) implies that for $(\tau, \eta)$ real, all eigenvalues $\xi_{d}$ of $\mathcal{G}^{11}(z, q, \tau-i \gamma, \eta)$ have nonzero imaginary part when $\gamma>0$. From (2.26)(a) it follows that these eigenvalues lie in the same fixed half plane, one of $\pm \Im \xi_{d}>0$.

(3) Suppose now that $\underline{\xi}_{d}$ is an eigenvalue of $\mathcal{G}^{11}(z, q, \underline{\tau}, \underline{\eta})$ for $(\underline{\tau}, \underline{\eta}) \in \mathbb{R}^{d} \backslash 0$. By (H3) there exists a unique eigenvalue $\beta\left(z, q, \eta, \xi_{d}\right)$ of $\mathcal{A}^{11}$, which is $C^{\bar{\infty}}$ in all arguments, analytic in $\xi_{d}$, of constant multiplicity, and such that

$$
\underline{\tau}+\beta\left(z, q, \underline{\eta}, \underline{\xi}_{d}\right)=0 .
$$

Note then that $\left(\underline{\tau}, \underline{\eta}, \underline{\xi}_{d}\right)$ is nonglancing, which means that

$$
\partial_{\xi_{d}} \beta \neq 0 \quad \text { at }\left(z, q, \underline{\eta}, \underline{\xi}_{d}\right)
$$

for otherwise the equation

$$
\underline{\tau}-i \gamma+\beta\left(z, q, \underline{\eta}, \xi_{d}\right)=0
$$

has roots in $\xi_{d}$ with $\Im \xi_{d}$ of both signs when $\gamma>0$, contradicting the conclusion of part (2). It follows that $-\underline{\xi}_{d}$ is a semisimple eigenvalue of $\mathcal{G}^{11}(z, q, \underline{\tau}, \underline{\eta})$ with multiplicity equal to that of $-\underline{\tau}$ as an eigenvalue of $\mathcal{A}^{11}\left(z, q, \underline{\eta}, \underline{\xi}_{d}\right)$ [32, Proposition 3.9]. Thus, the assertion of semisimplicity and constant multiplicity in (2.26)(b) follows from that in (H3).

(4) The ability to choose $Z$ as in (2.27) is immediate from compactness.

Remark 2.11. - (1) Hypothesis (H6) is the starting point for constructing shock solutions of the inviscid equation. The construction of profiles as in (H7) is the first step in the analysis of the viscous perturbation. We refer to $[8,25]$ for the construction of profiles for the Navier-Stokes equations. For example, Gilbarg shows that for a convex pressure law, profiles exist for shocks of any strength. See [25,33] for construction of small-amplitude profiles of general systems.

(2) Following $[24,38]$ we may take advantage of the divergence form of (2.22), integrating from $-\infty$ to $z$ to express the profile equation as an algebraic relation

$$
\tilde{f}_{d}^{1}(w, \nu)-\tilde{f}_{d}^{1}\left(u_{-}, \nu\right) \equiv 0,
$$

combined with the reduced ODE

$$
\partial_{z} w^{2}=\left(\widetilde{B}_{d, d}^{22}\right)^{-1}\left(\tilde{f}_{d}^{2}(w, \nu)-\tilde{f}_{d}^{2}\left(u_{-}, \nu\right)\right) .
$$

Since $\widetilde{A}_{d}^{11}=\tilde{f}_{d}^{1^{\prime}}$ is invertible by the first part of (H8), we may solve for $w_{1}$ as a function of $w_{2}$ in (2.34), reducing the algebro-differential system (2.34)-(2.35) to a standard ODE in the variable $w_{2}$.

(3) Hypothesis (H8), especially through its consequence, Proposition 2.10, plays an important part in the high frequency estimate. In the estimate (3.97)(a) for the hyperbolic block, it is essential that the eigenvalues of $\mathcal{A}^{11}$ be nonglancing (2.32). One can show that if the eigenvalues of $\mathcal{A}^{11}$ are nonglancing and (H8) fails to hold, then constant multiplicity for $\mathcal{A}^{11}$ (H3) fails.

(H8) is satisfied in physical examples, and it holds in particular for the Navier-Stokes equations. More generally, consider the case where $s=N-1$, so that $L^{11}$ is a vector field

$$
L^{11}=A_{0}^{11}(u) \partial_{t}+\sum_{j=1}^{d} A_{j}^{11}(u) \partial_{j}
$$


with $A_{j}^{11} \in \mathbb{R}$. In this case (H8) reduces to the condition that

$$
\widetilde{A}_{d}^{11}\left(W_{0}(z, p), \nu(h)\right)=\sum_{j=0}^{d} \nu_{j} A_{j}^{11}\left(W_{0}(z, p)\right) \neq 0
$$

for all $p \in \mathcal{C}$ and $z \in \mathbb{R} \cup \pm \infty$.

For the Navier-Stokes equations,

$$
L^{11}=\partial_{t}+\sum_{j=1}^{d} v_{j} \partial_{j}
$$

and the condition reads

$$
h_{0} \neq v_{d}-\sum_{j=1}^{d-1} h_{j} v_{j} \quad \text { along the profile, }
$$

where, with $H=\left|\left(-h_{1}, \ldots,-h_{d-1}, 1\right)\right|$,

$$
h_{0} / H \quad \text { and } \quad v_{n}=\left(v_{d}-\sum_{j=1}^{d-1} h_{j} v_{j}\right) / H
$$

are the speed of the shock and fluid speed normal to the shock respectively. One can show that (2.38) is a consequence of the profile equations; see [37, Appendix A.1]. For weak shocks this condition is clear, since Lax shocks are associated to acoustic modes and their speed is $\approx v_{n} \pm c$, where $c$ is the sound speed.

Definition 2.12. - A solution $w$ of the connection problem (2.34)-(2.35), (2.23) is called transversal if the unstable manifold of (2.35) at $u_{2}^{-}$and the stable manifold of (2.35) at $u_{2}^{+}$ intersect transversally along $w_{2}$.

PROPOSITION 2.13. - (See $[24,38]$.) Suppose that $w$ is a shock profile associated to a planar Lax shock $p=\left(u^{-}, u^{+}, h\right)$. Then $w$ converges exponentially in all derivatives to its end states $u^{ \pm}$ and $\partial_{z} w_{2}(z) \neq 0$ for all $z \in \mathbb{R}$. Moreover, if $w$ is transversal, then it is unique up to translation.

Proof. - Evaluating $\partial_{w_{2}} w_{1}$ in (2.34) using the Implicit Function Theorem, we find that the linearization of the right-hand side of (2.35) about the endstates $w_{2}^{ \pm}$is

$$
\left(\widetilde{B}_{d, d}^{22}\right)^{-1}\left(\widetilde{A}_{d}^{22}-\widetilde{A}_{d}^{21}\left(\widetilde{A}_{d}^{11}\right)^{-1} \widetilde{A}_{d}^{12}\right) .
$$

By Assumption (H2) and Assumption (H8) we have

$$
\operatorname{det}\left(\left(\widetilde{B}_{d, d}^{22}\right)^{-1}\left(\widetilde{A}_{d}^{22}-\widetilde{A}_{d}^{21}\left(\widetilde{A}_{d}^{11}\right)^{-1} \widetilde{A}_{d}^{12}\right)\right)=\operatorname{det} \widetilde{A}_{d} /\left(\operatorname{det} \widetilde{B}_{d, d}^{22} \operatorname{det} \widetilde{A}_{d}^{11}\right) \neq 0,
$$

so that $w_{2}^{ \pm}$are nondegenerate rest points of (2.35). With Assumption (H5) this implies that (2.39) has no purely imaginary eigenvalues. Therefore, $w_{2}^{ \pm}$are hyperbolic rest points, from which exponential decay follows by classical ODE theory [3]. Likewise, $\partial_{z} w_{2}(z) \neq 0$ follows from uniqueness of solutions of ODE. Uniqueness up to translation follows from the relation

$$
s_{+}+s_{-}-s=N_{+}+N_{-}-N \quad[37, \text { Appendix A.2] }
$$

$4^{\text {e }}$ SÉRIE - TOME $39-2006-\mathrm{N}^{\circ} 1$ 
where $s_{+}$denotes the number of eigenvalues with negative real part of (2.39) at $w_{2}^{+}$and $s_{-}$ denotes the number of eigenvalues with positive real part of (2.39) at $w_{2}^{-}$; this quantity equals one for Lax shocks.

Remark 2.14. - (1) Profiles for viscous shock solutions of the Navier-Stokes equations are transversal if they exist [24]. Such shocks are in general extreme shocks.

(2) We prove the relation (2.41) in Corollary 3.45.

From Proposition 2.13, we obtain as in the proof of Proposition 2.6, [12] the following local verification of $(\mathrm{H} 7)$.

PROPOSITION 2.15. - (See [12].)

(i) Suppose that $p$ is a planar Lax shock. Then there is a neighborhood $\omega$ of $p$ in $\mathcal{U} \times \mathcal{U} \times \mathbb{R}^{d}$ such that the set of shocks in $\omega$ form a smooth manifold $\mathcal{C}$ of dimension $\bar{N}+d$ and each $p \in \mathcal{C}$ is a Lax shock.

(ii) Suppose in addition that $\underline{w}$ is a shock profile associated to $\underline{p}$ and that $\underline{w}$ is transversal. Then, shrinking $\omega$ if necessary, there is a $C^{\infty}$ mapping $W_{0}$ from $\mathbb{R} \times \mathcal{C}$ to $\mathcal{U}^{*} \subset \mathbb{R}^{N}$ such that $W_{0}(z, p)=\underline{w}(z)$ and for all $p=\left(u^{-}, u^{+}, h\right) \in \mathcal{C}, W_{0}(\cdot, p)$ is a shock profile associated to $p$. This connection is unique, up to a translation in $z$ by a smooth shift $k(p)$.

In (2.24) and Proposition 2.10 we have already introduced a more general type of profile. We formalize that notion in the next definition.

Definition 2.16. - Let $p=\left(u^{-}, u^{+}, h\right) \in \mathcal{C}$ be as in (H6) and let $W_{0}(z, p)$ be a shock profile as in (H7). For parameters $\left(u^{\prime}, h^{\prime}\right) \in \mathbb{R}^{N} \times \mathbb{R}^{d}$ and $q=\left(p, u^{\prime}, h\right)$ we define the function $W(z, q)=W_{0}(z, p)+u^{\prime}$ to be a profile associated to the front $h+h^{\prime}$ if

(a) $W(z, q) \in \mathcal{U}^{*}$ for all $z$;

(b) there exists $Z>0$ such that $|z| \geqslant Z \Rightarrow W(z, q) \in \mathcal{U}$;

(c) for $|z| \geqslant Z, \bar{A}_{d}(z, q)$ is invertible;

(d) for all $z$ one of $\pm \bar{A}_{d}^{11}(z, q)$ has only strictly positive eigenvalues.

Remark 2.17. - From Proposition 2.10 we see that for parameters $\left(u^{\prime}, h^{\prime}\right)$ contained in a small enough neighborhood of $0, W(z, q)$ as defined above satisfies conditions (a)-(d) with a $Z$ that can be taken independent of $q$. Moreover, it follows as in [12, Proposition 2.6] that for some $\theta>0$ we have estimates

$$
\left|\partial_{z}^{k} \partial_{q}^{\alpha} W(z, q)\right| \leqslant C_{k, \alpha} e^{-\theta|z|} \quad \text { on } \pm z \geqslant 0 .
$$

Note that we distinguish between "shock profiles" as in (H7) and more general profiles as in Definition 2.16.

\subsubsection{The uniform Evans condition}

For a fixed $q=\left(p, u^{\prime}, h^{\prime}\right)$ we consider a profile $W(z, q)$ as in Definition 2.16. We consider the linearization of Eq. (2.20) around

$$
w^{\varepsilon}(t, y, x)=W(x / \varepsilon, q), \quad \psi(t, y)=\left(h+h^{\prime}\right) \cdot(t, y) .
$$

For simplicity, we have changed the notation $\tilde{x}$ to $x$.

We first compute the "partially linearized" operator with respect to $u$ alone (we compute the fully linearized operator in Section 2.4, following). This has the form

$$
L \dot{u}:=-\varepsilon \partial_{x}\left(\widetilde{B}_{d, d} \partial_{x} \dot{u}\right)+\partial_{x}\left(A^{\sharp} \dot{u}\right)+\frac{1}{\varepsilon} M^{\sharp} \dot{u}
$$


where

$$
\left\{\begin{array}{l}
A^{\sharp} v=\widetilde{A}_{d} v-\sum_{j=1}^{d-1}\left(\widetilde{B}_{j, d}+\widetilde{B}_{d, j}\right) \varepsilon \partial_{j} v-\left(\nabla_{u} \widetilde{B}_{d, d} \cdot v\right) \partial_{z} W, \\
M^{\sharp} v=A_{0} \varepsilon \partial_{t} v+\sum_{j=1}^{d-1} A_{j}^{\sharp} \varepsilon \partial_{j} v-\sum_{j=1, k}^{d-1} B_{j, k} \varepsilon^{2} \partial_{j} \partial_{k} v,
\end{array}\right.
$$

with

$$
\begin{aligned}
A_{j}^{\sharp} v & =A_{j} v-\left(\nabla_{u} \widetilde{B}_{j, d} \cdot v\right) \partial_{z} W+\left(\nabla_{u} \widetilde{B}_{j, d} \cdot \partial_{z} W\right) v, \quad j=1, \ldots, d-1, \\
\widetilde{B}_{j, d}(u, \nu) & =\sum_{k=1}^{d} \nu_{k} B_{j, k}(u), \quad \widetilde{B}_{d, j}(u, \nu)=\sum_{k=1}^{d} \nu_{k} B_{k, j}(u), \quad j=1, \ldots, d-1,
\end{aligned}
$$

where $\nu(h)=\left(-h_{0},-h_{1}, \ldots,-h_{d-1}, 1\right)$ and matrix coefficients are evaluated at $u=W(x / \varepsilon, q)$. The coefficients are smooth functions of $q$ and $z=x / \varepsilon$, and $A^{\sharp}$ and $M^{\sharp}$ are differential operators in $\varepsilon \partial_{t}$ and $\varepsilon \partial_{y}$.

Since the coefficients of $L$ are independent of the tangential space-time variables $(t, y)$, one can perform a Fourier-Laplace transform with respect to $(t, y)$. This leads to symbols $\mathcal{A}(z, q, \zeta)$ and $\mathcal{M}(z, q, \zeta)$, depending on $(z, q)$ and $\zeta=(\tau, \eta, \gamma) \in \mathbb{R} \times \mathbb{R}^{d-1} \times \mathbb{R}$, obtained by evaluating the coefficients at $(z, q)$ and replacing $\partial_{j}$ and $\partial_{t}$ in the definitions above by $i \eta_{j}, j=1, \ldots, d-1$, and $\gamma+i \tau$ respectively. Denoting by $\hat{u}$ (respectively, $\hat{f}$ ) the Fourier-Laplace transform of $\dot{u}$ (respectively, $L \dot{u}$ ), one has:

$$
\hat{f}(x, \hat{\zeta})=-\varepsilon \partial_{x}\left(\mathcal{B}\left(\frac{x}{\varepsilon}, q\right) \partial_{x} \hat{u}\right)+\partial_{x}\left(\mathcal{A}\left(\frac{x}{\varepsilon}, q, \varepsilon \hat{\zeta}\right) \hat{u}\right)+\frac{1}{\varepsilon} \mathcal{M}\left(\frac{x}{\varepsilon}, q, \varepsilon \hat{\zeta}\right) \hat{u}
$$

Denote by $\hat{L}$ the operator in the right-hand side acting on $\hat{u}$. It is then natural to rescale the variables. After setting

$$
\zeta=\varepsilon \hat{\zeta}, \quad z=x / \varepsilon, \quad u^{*}(z, \zeta)=\hat{u}(x, \hat{\zeta}), \quad f^{*}(z, \zeta)=\varepsilon \hat{f}(x, \hat{\zeta})
$$

and

$$
\mathcal{L}\left(z, q, \zeta, \partial_{z}\right) u^{*}:=-\partial_{z}\left(\mathcal{B}(z, q) \partial_{z} u^{*}\right)+\partial_{z}\left(\mathcal{A}(z, q, \zeta) u^{*}\right)+\mathcal{M}(z, q, \zeta) u^{*},
$$

Eq. (2.46) reads

$$
f^{*}=\mathcal{L}\left(z, q, \zeta, \partial_{z}\right) u^{*}
$$

Dropping the stars, we now consider the well posedness of the equation

$$
\mathcal{L}\left(z, q, \zeta, \partial_{z}\right) u=f
$$

This is a degenerate second order differential equation, and the equation is equivalent to the transmission problem where one looks for solutions $u^{+}$and $u^{-}$on $\{z \geqslant 0\}$ and $\{z \leqslant 0\}$ separately, which satisfy the transmission conditions

$$
u^{-}(0)=u^{+}(0), \quad \partial_{z} u_{2}^{-}(0)=\partial_{z} u_{2}^{+}(0) .
$$

Note that these are equivalent to (1.11), by the block structure assumption (2.5).

Definition 2.18. - Given a profile $W(z, q)$ as in Definition 2.16, we denote by $\mathbb{E}^{+}(q, \zeta)$ (respectively, $\left.\mathbb{E}^{-}(q, \zeta)\right)$ the set of initial data $\left(u(0), \partial_{z} u_{2}(0)\right)$ such that the corresponding solution of $\mathcal{L}\left(z, q, \zeta, \partial_{z}\right) u=0$ on $\{z \geqslant 0\}$ (respectively, $\{z \leqslant 0\}$ ) is bounded as $z$ tends to $+\infty$ (respectively, $-\infty)$. 
In the sequel, we denote by $\overline{\mathbb{R}}_{+}^{d+1}$ the set of parameters $\zeta=(\tau, \eta, \gamma) \in \mathbb{R} \times \mathbb{R}^{d-1} \times \mathbb{R}$ such that $\gamma \geqslant 0$ and by $\overline{\mathbb{R}}_{+}^{d+1} \backslash\{0\}$ the set of $\zeta \neq 0$ with $\gamma \geqslant 0$. The proof of the next lemma is given in Section 3.3.

LEMMA 2.19. - Assume (H0)-(H2) and (H5)-(H8). Let $W(z, q)$ be a profile as in Definition 2.16. Then for

$$
q=\left(p, u^{\prime}, h^{\prime}\right) \in \mathcal{Q}:=\mathcal{C} \times \mathcal{O},
$$

with $\left(u^{\prime}, h^{\prime}\right)$ in a small enough neighborhood $\mathcal{O}$ of $0, \mathbb{E}^{+}(q, \zeta)$ and $\mathbb{E}^{-}(q, \zeta)$ are smooth vector bundles of fixed dimensions $K, N+s-K$ in $\mathbb{C}^{N+s}$ over $\mathcal{Q} \times \mathbb{R}_{+}^{d+1} \backslash\{0\}$. In fact, $K=N$ when the eigenvalues of $\bar{A}_{d}^{11}(z, q)$ are all positive, and $K=s$ when those eigenvalues are all negative.

There are nontrivial bounded solutions of $\mathcal{L} u=0$ if and only if $\mathbb{E}^{+} \cap \mathbb{E}^{-} \neq\{0\}$. The distance between these two spaces can be measured via the Evans' function

$$
D(q, \zeta)=\operatorname{det}\left(\mathbb{E}^{+}(q, \zeta), \mathbb{E}^{-}(q, \zeta)\right)
$$

where the determinant is obtained by taking any orthonormal basis in the given spaces. Note that, by Lemma 2.19 , the function $D$ is smooth on $\mathcal{Q} \times \mathbb{R}_{+}^{d+1} \backslash\{0\}$.

There is an alternate way of computing the Evans function $D$. Considering the transmission problem as a boundary problem, the natural space of initial data of bounded solutions is $\mathbb{E}^{-} \times \mathbb{E}^{+} \subset \mathbb{C}^{N+s} \times \mathbb{C}^{N+s}$. Its dimension is $N+s$. The boundary condition can be written $\Gamma\left(U^{-}, U^{+}\right)=0$ where $\Gamma$ is the mapping $\left(U^{-}, U^{+}\right) \mapsto U^{+}-U^{-}$from $\mathbb{C}^{N+s} \times \mathbb{C}^{N+s}$ to $\mathbb{C}^{N+s}$. Thus $\operatorname{dim} \operatorname{ker} \Gamma=N+s$ and

$$
D(q, \zeta)=\operatorname{det}\left(\mathbb{E}^{-}(q, \zeta) \times \mathbb{E}^{+}(q, \zeta), \operatorname{ker} \Gamma\right)
$$

The weak Evans condition requires that $D$ does not vanish when $\zeta \neq 0$ and $\gamma \geqslant 0$. The uniform condition requires in addition an optimal control when $\zeta$ is small or large. It turns out that for large $\zeta$ appropriate control follows already from the assumptions (H0)-(H8), and so no explicit assumption is necessary in this regime; see Remark 3.29. For small $\zeta$, we know from [37,39] that the determinant $D$ is $O(|\zeta|)$. Following [36] the uniform stability condition reads:

DEFINITION 2.20 (Stability conditions). -

(i) The shock profile $W_{0}(z, p)$ associated to a Lax shock $q=(p, 0)$ is spectrally stable (satisfies the weak Evans condition) if the Evans function $D(p, 0, \zeta)$ does not vanish for $\zeta \in \overline{\mathbb{R}}_{+}^{d+1} \backslash\{0\}$.

(ii) It is uniformly stable (satisfies the uniform Evans condition) if in addition there is a positive constant $c$ such that for all $\zeta \in \overline{\mathbb{R}}_{+}^{d+1} \backslash\{0\}$ with $|\zeta| \leqslant 1$,

$$
|D(p, 0, \zeta)| \geqslant c|\zeta|
$$

Assumption $2.21(\mathrm{H} 9)$. - For all $p \in \mathcal{C}$, the planar profile $W_{0}(z, p)$ is uniformly stable.

Remark 2.22. - In [34] it is shown that profiles associated to a large class of weak Lax shocks, including weak shocks for the Euler equations, are uniformly stable. See also [6] for a similar result in the one-dimensional case.

The following proposition extends a result of [39] in the case of artificial viscosity to the real viscosity setting. The proposition refers to Majda's uniform stability condition for inviscid shocks, which is recalled in Appendix A along with the proof of the proposition. 
PROPOSITION 2.23. - Suppose that assumptions $(\mathrm{H} 0)-(\mathrm{H} 2)$ and $(\mathrm{H} 4)-(\mathrm{H} 8)$ are satisfied and that $W_{0}$ is a shock profile associated to a planar Lax shock $p$.

(i) If $W_{0}$ is uniformly stable, then $W_{0}$ is transversal and the planar shock $p$ is uniformly stable in the sense of Majda [26].

(ii) Conversely, if $W_{0}$ is transversal and the shock $p$ is uniformly stable, then (2.55) holds for $\zeta \in \overline{\mathbb{R}}_{+}^{d+1} \backslash\{0\}$ small enough.

COROLLARY 2.24. - Under the assumptions of Proposition 2.23, a profile $W_{0}$ associated to a Lax shock $p$ is uniformly stable if and only if:

(i) it is spectrally stable,

(ii) $W_{0}$ is transversal,

(iii) $p$ is uniformly stable in the sense of Majda.

Remark 2.25. - We shall adopt the convention from now on that hypotheses (H0)-(H1) and (H6)-(H7) are automatically assumed in every theorem, proposition, etc. of the paper. Thus, in Proposition 3.8 for example, where the only explicitly stated assumption is (H2), our intention is to highlight the fact that $(\mathrm{H} 2)$ is the only extra assumption needed beyond the automatically assumed ones.

\subsection{The fully linearized equations}

The vanishing of the Evans function associated to a Lax shock profile $W_{0}$ at $\zeta=0$ is the main source of difficulty in the low frequency analysis. It reflects the translational degeneracy in the partially linearized operator $\mathcal{L}$ expressed by the fact

$$
\mathcal{L}\left(z, q, 0, \partial_{z}\right) \partial_{z} W_{0}=0 \quad \text { when } q=(p, 0) .
$$

This degeneracy leads to an $L^{2}$ estimate for the transmission problem (2.50), (2.51) that is too weak for our purposes here. In addition, having introduced the extra unknown $\psi^{\varepsilon}$ in (1.10), we should expect the transmission problem (1.10), (1.11) to be underdetermined without some extra boundary (or transmission) condition. The key to the low frequency stability analysis, here as in [12], is to work with the fully linearized problem and to choose the extra boundary condition in a way that removes the translational degeneracy in the low frequency regime. This strategy then commits us to working with the fully linearized equations in the medium and high frequency regimes as well, even though the partially linearized problem is well behaved there.

Consider again a profile $W(z, q)$ with $q=\left(p, u^{\prime}, h^{\prime}\right)$. The fully linearized equation from (1.10) around $w^{\varepsilon}=W(x / \varepsilon, q), \psi(t, y)=\left(h+h^{\prime}\right) \cdot(t, y)$ reads

$$
L \dot{u}-K \dot{\psi}=\dot{f}
$$

where $L$ is given by (2.44) and

$$
\begin{aligned}
K \dot{\psi}= & \partial_{t} \dot{\psi} \partial_{x} f_{0}\left(w^{\varepsilon}\right)+\sum_{j=1}^{d-1} \partial_{j} \dot{\psi} \partial_{x} f_{j}\left(w^{\varepsilon}\right) \\
& -\sum_{j=1}^{d-1} \varepsilon \partial_{j} \dot{\psi} \partial_{x}\left(\left(\widetilde{B}_{j, d}+\widetilde{B}_{d, j}\right) \partial_{x} w^{\varepsilon}\right)-\varepsilon \sum_{j, k=1}^{d-1} \partial_{j} \partial_{k} \dot{\psi} B_{j, k} \partial_{x} w^{\varepsilon}
\end{aligned}
$$

The key idea introduced in [12] is to consider the problem (2.57) with transmission conditions

$$
[\dot{u}]=0, \quad\left[\partial_{x} \dot{u}_{2}\right]=0, \quad \partial_{t} \dot{\psi}-\varepsilon \Delta_{y} \dot{\psi}+\ell \cdot \dot{u}_{2}=0 \quad \text { on } x=0,
$$

$4^{\text {e }}$ SÉRIE - TOME $39-2006-\mathrm{N}^{\circ} 1$ 
for $\ell=\ell(q) \in \mathbb{R}^{s}$. The special choice of the heat operator in the extra boundary condition is not essential. It can be replaced by any parabolic operator of the same type, possibly depending on $p$. There is also a large freedom in the choice of $\ell$. We assume $\ell(q)$ satisfies

$$
\ell(q) \cdot \partial_{z} W_{2}(0, q)>0 .
$$

Such a choice is always possible for profiles close enough to shock profiles since $\partial_{z} W_{2}(0, q) \neq 0$ when $q=(p, 0)$ by Proposition 2.13.

The operators $L$ and $K$ are closely related. Denote by $\mathcal{E}(u, \psi)$ the left-hand side of (1.10) and consider for a moment the full and partial linearizations of (1.10) about an arbitrary choice of $(u, \psi)$, together with the original transmission conditions:

$$
\begin{array}{lll}
\text { (a) } \mathcal{E}_{u}^{\prime}(u, \psi) \dot{u}+\mathcal{E}_{\psi}^{\prime}(u, \psi) \dot{\psi}=\dot{f}, & {[\dot{u}]=0,} & {\left[\partial_{x} \dot{u}_{2}\right]=0,} \\
\text { (b) } \mathcal{E}_{u}^{\prime}(u, \psi) \dot{u}=\dot{f}, \quad[\dot{u}]=0, & {\left[\partial_{x} \dot{u}_{2}\right]=0,}
\end{array}
$$

where $\mathcal{E}_{u}^{\prime}$ and $\mathcal{E}_{\psi}^{\prime}$ are the linearizations of $\mathcal{E}$ with respect to $u$ and $\psi$ respectively.

LEMMA 2.26. - We have

$$
\mathcal{E}_{u}^{\prime}(u, \psi) \dot{u}+\mathcal{E}_{\psi}^{\prime}(u, \psi) \dot{\psi}=\mathcal{E}_{u}^{\prime}(u, \psi)\left(\dot{u}-\dot{\psi} \partial_{x} u\right)+\dot{\psi} \partial_{x} \mathcal{E}(u, \psi) .
$$

Proof. - Denoting by $\mathcal{F}(u)$ the left-hand side of Eq. (1.1) in the original coordinates, and by * the substitution $u^{*}(t, y, x)=u(t, y, x-\psi(t, y))$, one has

$$
\mathcal{F}\left(u^{*}\right)=\{\mathcal{E}(u, \psi)\}^{*} .
$$

Through linearization, one has $\delta\left(u^{*}\right)=\left(\delta u-\delta \psi \partial_{x} u\right)^{*}$. Moreover, differentiating in $u$ alone, one checks that $\left(\mathcal{E}_{u}^{\prime}(u, \psi) v\right)^{*}=\mathcal{F}_{u}^{\prime}\left(u^{*}\right)(v)^{*}$. Linearizing (2.63) implies (2.62).

Remark 2.27. - The identity (2.62) was pointed out by S. Alinhac [1] along with the role of what he called "the good unknown" $\dot{u}-\dot{\psi} \partial_{x} u$. Consider the example where $u=W_{0}\left(\frac{x}{\varepsilon}, p\right)$ and $\psi=h \cdot(t, y)$ are an exact solution of the problem (1.10), (1.11). In this case, the error term $\partial_{x} \mathcal{E}(u, \psi)$ is exactly equal to zero in the right-hand side of (2.62) and the original transmission conditions for $\dot{u}$ and $\dot{v}=\dot{u}-\dot{\psi} \partial_{x} u$ are equivalent. Hence, the transmission problems (2.61)(a) for $\dot{u}$ and (2.61)(b) for $\dot{v}$ are equivalent. This observation is useful in the medium and high frequency regions, where the partially linearized problem $(2.61)(\mathrm{b})$ is well-behaved. There we may prove estimates for the good unknown $\dot{v}$ satisfying (2.61)(b), and then use the extra boundary condition

$$
\left(\partial_{t}-\varepsilon \Delta_{y}\right) \dot{\psi}+\dot{\psi}\left(\ell \cdot \partial_{x} u_{2}\right)=-\ell \cdot \dot{v}_{2} \quad \text { on } x=0,
$$

to estimate $\dot{\psi}$ after estimating the trace of $v_{2}$.

This approach has to be modified in the small frequency region because of the translational degeneracy at $\zeta=0$. By making a more subtle choice of good unknown as explained in Section 3.3 , one can again reduce to proving estimates for the partially linearized operator $\mathcal{E}_{u}^{\prime}$. The original transmission conditions are replaced by new (pseudodifferential) boundary conditions arising from (2.59) on the good unknown, and these conditions have the effect of removing the translational degeneracy.

The coefficients of $K(2.58)$ are independent of $(t, y)$, so we again perform a Fourier-Laplace transform with respect to $(t, y)$. Denote by $g$ the additional term $-K \dot{\psi}$ in (2.57) and by $\hat{\psi}$ and $\hat{g}$ the Fourier-Laplace transforms of $\dot{\psi}$ and $g$ respectively. Parallel to (2.46) there holds 


$$
\hat{g}(x, \hat{\zeta})=-\frac{1}{\varepsilon^{2}} \hat{\psi}(\hat{\zeta}) \mathcal{K}\left(\frac{x}{\varepsilon}, q, \varepsilon \hat{\zeta}\right)
$$

where

$$
\begin{aligned}
\mathcal{K}(z, q, \zeta)= & \partial_{z} f_{0}(W)(\gamma+i \tau)+\sum_{j=1}^{d-1} \partial_{z} f_{j}(W) i \eta_{j} \\
& -\sum_{j=1}^{d-1} \partial_{z}\left(\left(\widetilde{B}_{j, d}+\widetilde{B}_{d, j}\right) \partial_{z} W\right) i \eta_{j}+\sum_{j, k=1}^{d-1} B_{j, k} \partial_{z} W \eta_{j} \eta_{k}
\end{aligned}
$$

and the coefficients are now evaluated at $u=W(z, q)$. The natural rescaling for $\hat{g}$ and $\hat{\psi}$, which supplements (2.47), is:

$$
g^{*}(z, \zeta)=\varepsilon \hat{g}(x, \hat{\zeta}), \quad \psi^{*}(\zeta)=\frac{1}{\varepsilon} \hat{\psi}(\hat{\zeta})
$$

so that

$$
g^{*}(z, \zeta)=-\psi^{*}(\zeta) \mathcal{K}(z, q, \zeta)
$$

Similarly, the Fourier-Laplace transform of the extra boundary condition reads

$$
\left(\hat{\gamma}+i \hat{\tau}+\varepsilon|\hat{\eta}|^{2}\right) \hat{\psi}(\hat{\zeta})+\ell \cdot \hat{u}_{2 \mid x=0}(\hat{\zeta})=0 .
$$

Adding up, after Fourier-Laplace transform and rescaling as in (2.47) and (2.66), we see that the linearized equations read:

$$
\begin{aligned}
& \mathcal{L}\left(z, q, \zeta, \partial_{z}\right) u^{*}-\psi^{*} \mathcal{K}(z, q, \zeta)=f^{*}, \\
& a(\zeta) \psi^{*}+\ell \cdot u_{2}^{*}(0)=0 \quad \text { on } z=0,
\end{aligned}
$$

with $a(\zeta)=\gamma+i \tau+|\eta|^{2}$.

LEMMA 2.28. - Given a profile $W(z, q)$ with $q=\left(p, u^{\prime}, h^{\prime}\right)$ as above, the following identity is satisfied:

$$
\mathcal{K}(z, q, \zeta)=\mathcal{L}\left(z, q, \zeta, \partial_{z}\right) \partial_{z} W+\partial_{z} \mathcal{P}\left(W, \nu\left(h+h^{\prime}\right)\right),
$$

where $\mathcal{P}(W, \nu):=\partial_{z}\left(\widetilde{B}_{d, d}(W, \nu) \partial_{z} W\right)-\partial_{z}\left(\tilde{f}_{d}(W, \nu)\right)$.

Proof. - This is easily checked by direct computation; it can also be deduced from the identity (2.62).

Remark 2.29. - In the case when $W_{0}$ is a shock profile associated to the front $h$, we have $\mathcal{P}\left(W_{0}, \nu(h)\right)=0$.

\section{Frozen coefficient $L^{2}$ estimates}

\subsection{Transmission problems depending on frequency}

Consider an approximate solution $\left(u_{a}^{\varepsilon, M}, \psi_{a}^{\varepsilon, M}\right)$ as in (1.12). It can be written

$$
\begin{aligned}
u_{a}^{\varepsilon, M}(x) & =W_{0}\left(\frac{x}{\varepsilon}, p(t, y)\right)+u^{\prime}(t, y, x, \varepsilon), \\
d \psi_{a}^{\varepsilon, M} & =d \psi^{0}(t, y)+h^{\prime}(t, y, x, \varepsilon),
\end{aligned}
$$

$4^{e}$ SÉRIE - TOME $39-2006-\mathrm{N}^{\circ} 1$ 
where

$$
p(t, y)=\left(u_{+}^{0}(t, y, 0), u_{-}^{0}(t, y, 0), d \psi^{0}(t, y)\right) \in \mathcal{C}
$$

is the given inviscid shock and $u^{\prime}, h^{\prime}$ are perturbations that can be read off from (1.12). We also allow $u^{\prime}$ and $h^{\prime}$ to include additional corrections of the form $\varepsilon^{L}\left(v_{n}, d \phi_{n}\right)$ like those that will appear later in the iteration scheme for the exact solution. Let us now freeze $q=\left(p, u^{\prime}, h^{\prime}\right)$ and consider the rescaled, Fourier-Laplace transformed, transmission problem (2.67):

$$
\begin{gathered}
\mathcal{L}\left(z, q, \zeta, \partial_{z}\right) u-\psi \mathcal{K}(z, q, \zeta)=f, \\
{[u]=0, \quad\left[\partial_{z} u_{2}\right]=0, \quad a(\zeta) \psi+\ell(q) \cdot u_{2}(0)=0 \quad \text { on } z=0 .}
\end{gathered}
$$

The problem (3.3) is a transmission problem in $z$ depending on $(q, \zeta)$ as parameters. In this part we show how to obtain estimates for this problem, uniform with respect to the parameters, in each of the three frequency regimes where $|\zeta|$ is small, medium, or large. This ODE question already contains most of the main difficulties. The frequency-dependent conjugators and symmetrizers constructed here will serve as symbols of the paradifferential operators we will use in the next part to obtain estimates for the variable coefficient linearized problem.

The following propositions give the frozen coefficient estimates in the three frequency regimes.

PRoposition 3.1 (High frequency).- Consider solutions $(u, \psi)$ of (3.3) where $q=$ $\left(p, u^{\prime}, h^{\prime}\right)$ with $p \in \mathcal{C}$ and $\left(u^{\prime}, h^{\prime}\right)$ small. There exist constants $R, \delta$ such that for $|\zeta| \geqslant R$, $0 \leqslant \gamma \leqslant \delta|\zeta|$, we have

$$
\begin{gathered}
(1+\gamma)\left\|u_{1}\right\|+\Lambda\left\|u_{2}\right\|+\left\|\partial_{z} u_{2}\right\|+\sqrt{1+\gamma}\left|u_{1}(0)\right|+\Lambda^{1 / 2}\left|u_{2}(0)\right| \\
+\Lambda^{-1 / 2}\left|\partial_{z} u_{2}(0)\right|+\Lambda^{5 / 2}\|\psi\| \leqslant C\left(\left\|f_{1}\right\|+\Lambda^{-1}\left\|f_{2}\right\|\right)
\end{gathered}
$$

where $\|f\|=|f|_{L^{2}(z)}$ and $\Lambda(\zeta)=\left(1+\gamma^{2}+\tau^{2}+|\eta|^{4}\right)^{1 / 4}$.

For $\gamma \geqslant \delta|\zeta|,|\zeta| \geqslant R$, we have the stronger estimate

$$
\begin{gathered}
|\zeta|\left\|u_{1}\right\|+\Lambda\left\|u_{2}\right\|+\left\|\partial_{z} u_{2}\right\|+\sqrt{|\zeta|}\left|u_{1}(0)\right|+\Lambda^{1 / 2}\left|u_{2}(0)\right| \\
+\Lambda^{-1 / 2}\left|\partial_{z} u_{2}(0)\right|+\Lambda^{5 / 2}\|\psi\| \leqslant C\left(\left\|f_{1}\right\|+\Lambda^{-1}\left\|f_{2}\right\|\right) .
\end{gathered}
$$

PROPOSITION 3.2 (Low frequency). - Consider solutions $(u, \psi)$ of $(3.3)$ where $q=\left(p, u^{\prime}, h^{\prime}\right)$ with $p \in \mathcal{C}$ and $\left(u^{\prime}, h^{\prime}\right)$ small. There exists a constant $\rho_{0}$ such that for $|\zeta| \leqslant \rho_{0}$ we have:

$$
\lambda^{2}\|u\|+\lambda\left\|\partial_{z} u_{2}\right\|+\lambda|u(0)|+\lambda\left|\partial_{z} u_{2}(0)\right|+\lambda|\zeta||\psi| \leqslant C\left\|f_{1}, f_{2}\right\|,
$$

where $\lambda(\zeta)=\left(\gamma+|\zeta|^{2}\right)^{1 / 2}$.

Proposition 3.3 (Medium frequency). - Consider solutions $(u, \psi)$ of (3.3) where $q=$ $\left(p, u^{\prime}, h^{\prime}\right)$ with $p \in \mathcal{C}$ and $\left(u^{\prime}, h^{\prime}\right)$ small and let $\rho_{0}$ and $R$ be as above. For $\rho_{0} \leqslant|\zeta| \leqslant R$ we have:

$$
\|u\|+\left\|\partial_{z} u_{2}\right\|+|u(0)|+\left|\partial_{z} u_{2}(0)\right|+|\psi| \leqslant C\left\|f_{1}, f_{2}\right\| .
$$

Remark 3.4. - As we show explicitly later, there is another route to the problem (3.3). Linearizing (1.10) about the approximate solution (3.3), one obtains, in addition to terms like those appearing in $L$ (2.44) and $K(2.58)$, a number of other terms that are small in the sense that they turn out to be negligible in the proof of the $L^{2}$ estimate (4.11). Throwing the small terms away leaves us with the principal part of the fully linearized operator. Freezing $q=\left(p, u^{\prime}, h^{\prime}\right)$ in the principal part, Fourier transforming, and rescaling yields (3.3). 


\subsection{High frequency estimate}

Our goal in this section is to prove the estimates (3.4) and (3.5). We begin with the more difficult case $0 \leqslant \gamma \leqslant \delta|\zeta|$.

\subsubsection{Reduction to the partially linearized case}

The estimate (3.4) will be deduced from an estimate for the partially linearized transmission problem:

$$
\begin{gathered}
\mathcal{L}\left(z, q, \zeta, \partial_{z}\right) u=f, \\
{[u]=0, \quad\left[\partial_{z} u_{2}\right]=0 \quad \text { on } z=0 .}
\end{gathered}
$$

PROPOSITION 3.5. - There exist constants $R$, $\delta$ such that for $0 \leqslant \gamma \leqslant \delta|\zeta|,|\zeta| \geqslant R$, solutions $u$ of (3.8) satisfy

$$
\begin{aligned}
& (1+\gamma)\left\|u_{1}\right\|+\Lambda\left\|u_{2}\right\|+\left\|\partial_{z} u_{2}\right\|+\sqrt{1+\gamma}\left|u_{1}(0)\right|+\Lambda^{1 / 2}\left|u_{2}(0)\right|+\Lambda^{-1 / 2}\left|\partial_{z} u_{2}(0)\right| \\
& \quad \leqslant C\left(\left\|f_{1}\right\|+\Lambda^{-1}\left\|f_{2}\right\|\right) .
\end{aligned}
$$

To derive estimate (3.4) from Proposition 3.5, define the good unknown

$$
u^{\#}=u-\psi \partial_{z} W
$$

for $(u, \psi)$ satisfying (3.3), and observe using Lemma 2.28

$$
\begin{aligned}
& \text { (a) } \mathcal{L} u^{\#}=f+\psi \partial_{z} \mathcal{P} \\
& \text { (b) }\left(a(\zeta)+\ell \cdot \partial_{z} W_{2}\right) \psi=-\ell \cdot u_{2}^{\#} \quad \text { on } z=0 .
\end{aligned}
$$

The coefficient of $\psi$ in (3.11)(b) is $\sim \Lambda^{2}$, and $\partial_{z} W$ is exponentially decaying as $z \rightarrow \pm \infty$, so the estimate (3.9) for $u^{\#}$ implies the estimate (3.4) for $(u, \psi)$. So now we concentrate on the proof of Proposition 3.5.

Remark 3.6. - The same good unknown is used to reduce the proof of the estimate (3.5) to showing the following estimate for (3.8):

$$
\begin{aligned}
& |\zeta|\left\|u_{1}\right\|+\Lambda\left\|u_{2}\right\|+\left\|\partial_{z} u_{2}\right\|+\sqrt{|\zeta|}\left|u_{1}(0)\right|+\Lambda^{1 / 2}\left|u_{2}(0)\right|+\Lambda^{-1 / 2}\left|\partial_{z} u_{2}(0)\right| \\
& \quad \leqslant C\left(\left\|f_{1}\right\|+\Lambda^{-1}\left\|f_{2}\right\|\right) .
\end{aligned}
$$

\subsubsection{Spectral properties of the symbol of $\mathcal{L}$}

We begin by writing out the explicit form of $\mathcal{L}\left(z, q, \zeta, \partial_{z}\right)$. We will use notation like that in (2.24), where $q=\left(p, u^{\prime}, h^{\prime}\right)$ and all matrix coefficients are evaluated at $(z, q)$, with the $(z, q)$ dependence entering entirely through $W(z, q)$ and $\nu\left(h+h^{\prime}\right)$. The matrices $\widetilde{A}_{d}, \widetilde{B}_{d, d}(2.18)$ and $\widetilde{B}_{j, d}, \widetilde{B}_{d, j}(2.45)$ are as before, and when convenient we write

$$
\begin{aligned}
\widetilde{A}_{j}=A_{j} & \text { for } j=1, \ldots, d-1, \\
\widetilde{B}_{j, k}=B_{j, k} & \text { for } j \leqslant d-1, k \leqslant d-1 .
\end{aligned}
$$

We have

$$
\begin{aligned}
\mathcal{L}\left(z, q, \zeta, \partial_{z}\right) u= & -\widetilde{B}_{d, d} \partial_{z}^{2} u+\left(\mathbb{A}_{d}-\sum_{j=1}^{d-1}\left(\widetilde{B}_{j, d}+\widetilde{B}_{d, j}\right) i \eta_{j}\right) \partial_{z} u \\
& +\left(E+A_{0}(i \tau+\gamma)+\sum_{j=1}^{d-1} \mathbb{A}_{j} i \eta_{j}+\sum_{j, k=1}^{d-1} \widetilde{B}_{j, k} \eta_{j} \eta_{k}\right) u
\end{aligned}
$$

$4^{\mathrm{e}}$ SÉRIE - TOME $39-2006-\mathrm{N}^{\circ} 1$ 
where

$$
\begin{aligned}
\mathbb{A}_{j} v & =\widetilde{A}_{j} v-\left(\partial_{z} W \cdot d_{u} \widetilde{B}_{d, j}\right) v-\left(v \cdot d_{u} \widetilde{B}_{j, d}\right) \partial_{z} W, \quad j=1, \ldots, d, \\
E v & =\left(v \cdot d_{u} \widetilde{A}_{d}\right) \partial_{z} W-\left(v \cdot d_{u} \widetilde{B}_{d, d}\right) \partial_{z}^{2} W-d_{u}^{2} \widetilde{B}_{d, d}\left(v, \partial_{z} W\right) \partial_{z} W .
\end{aligned}
$$

We also set $\overline{\mathcal{L}}=A_{0}^{-1} \mathcal{L}$ and, for $\xi \in \mathbb{R}^{d}$, write its symbol

$$
\begin{aligned}
\overline{\mathcal{L}}(z, q, \tau-i \gamma, \xi) & =(i \tau+\gamma) I+\sum_{j=1}^{d} \overline{\mathbb{A}}_{j} i \xi_{j}+\sum_{j, k=1}^{d} \bar{B}_{j, k} \xi_{j} \xi_{k}+\bar{E}, \\
& =(i \tau+\gamma) I+i \overline{\mathbb{A}}(z, q, \xi)+\bar{B}(z, q, \xi)+\bar{E}(z, q),
\end{aligned}
$$

where now

$$
\bar{A}_{j}=A_{0}^{-1} \widetilde{A}_{j}, \quad \overline{\mathbb{A}}_{j}=A_{0}^{-1} \mathbb{A}_{j}, \quad \bar{B}_{j, k}=A_{0}^{-1} \widetilde{B}_{j, k}, \quad \bar{E}=A_{0}^{-1} E .
$$

As before set

$$
\bar{A}(z, q, \xi)=\sum_{j=1}^{d} \bar{A}_{j} \xi_{j} .
$$

Remark 3.7. - (1) In the statement of hypotheses (H2)-(H5), the matrices $\bar{A}_{j}, \bar{B}_{j, k}$ are defined differently from (3.17) (for example, $\widetilde{A}_{d}$ in (3.17) is replaced by $A_{d}$ in the original definition (2.13) of $\bar{A}_{d}$ ). However, it is easy to check that whenever (H2)-(H5) hold for those matrices with their original definitions, the same hypotheses hold when the matrices are defined as in (3.17).

(2) We shall prove the estimate (3.9) by separate arguments for the regions $0 \leqslant \gamma \leqslant \delta|\zeta|$ and $\gamma \geqslant \delta|\zeta|$. In the first region we may not treat $E v$ as a negligible lower order term.

(3) Observe that $\overline{\mathbb{A}}$ and $\bar{A}$ are functions of $(z, q, \xi)$, while $\overline{\mathbb{A}}_{j}$ and $\bar{A}_{j}$ are functions of $(z, q)$.

We will label each proposition, corollary, etc., in this section with either a $(\mathrm{P})$ or an $(\mathrm{E})$ to indicate, respectively, either that it holds all along the profile (i.e., for all $z$ ) or just near the endstates (i.e., just for $|z|$ sufficiently large).

The first proposition will allow us to reformulate the strict dissipativity condition (H5) and verify it for $\overline{\mathcal{L}}$ when $|\xi|$ is large enough. Throughout this section we let $\mathcal{O}$ be a bounded open neighborhood of 0 as in Proposition 2.10, and for $q=\left(p, u^{\prime}, h^{\prime}\right)$ with

$$
p \in \mathcal{C}, \quad\left(u^{\prime}, h^{\prime}\right) \in \mathcal{O},
$$

we take $W(z, q)$ to be a profile in the sense of Definition 2.16.

Proposition $3.8(\mathrm{P})$. - (a) Assume (H2) (ellipticity of the block $\bar{B}^{22}(z, q, \xi)$ ) and (3.19). For $|\xi|$ large enough there are $C^{\infty}$ matrices $V(z, q, \xi)$, symbols of degree 0 in $\xi$, such that

$$
V^{-1}(i \overline{\mathbb{A}}+\bar{B}+\bar{E}) V=\left(\begin{array}{cc}
\widehat{A}^{11} & 0 \\
0 & \widehat{B}^{22}
\end{array}\right)
$$

with

$$
\begin{aligned}
\widehat{A}^{11}(z, q, \xi) & =i \overline{\mathbb{A}}^{11}(z, q, \xi)+\left(\overline{\mathbb{A}}^{12}\left(\bar{B}^{22}\right)^{-1} \overline{\mathbb{A}}^{21}+\bar{E}^{11}\right)+\mathrm{O}\left(|\xi|^{-1}\right), \\
\widehat{B}^{22}(z, q, \xi) & =\bar{B}^{22}(z, q, \xi)+\mathrm{O}(|\xi|), \\
V(z, q, \xi) & =I+\left(\begin{array}{cc}
0 & i \overline{\mathbb{A}}^{12}\left(\bar{B}^{22}\right)^{-1} \\
-i\left(\bar{B}^{22}\right)^{-1} \overline{\mathbb{A}}^{21} & 0
\end{array}\right)+\mathrm{O}\left(|\xi|^{-2}\right) .
\end{aligned}
$$

(b) The same result holds when $\bar{E}$ is set equal to zero and $\overline{\mathbb{A}}$ is replaced by $\bar{A}$. 
Proof. - A direct computation and simple perturbation argument shows that (3.20) holds for a $V$ of the given form.

COROllary $3.9(\mathrm{P})$. - Assume (H2) and (3.19). There exist $R>0$ and $c>0$ such that for $|\xi| \geqslant R$, the eigenvalues $\lambda$ of $i \bar{A}(z, q, \xi)+\bar{B}(z, q, \xi)$ satisfy $\Re \lambda>c$ if and only if

$$
\operatorname{spec}\left\{i \bar{A}^{11}(z, q, \xi)+\bar{A}^{12}\left(\bar{B}^{22}\right)^{-1} \bar{A}^{21}\right\} \subset\{\Re \lambda>c\} .
$$

Proof. - Given (H2), this follows from part (b) of the above proposition.

COROLlary 3.10 (E). - Assume (H2), (H5), and (3.19). There exist $R>0$ and $Z_{1} \geqslant Z$ such that for $|\xi| \geqslant R$ and $|z| \geqslant Z_{1}$, the following equivalent properties hold:

$$
\begin{aligned}
& \text { (a) } \operatorname{spec}\left\{i \bar{A}^{11}(z, q, \xi)+\bar{A}^{12}\left(\bar{B}^{22}\right)^{-1} \bar{A}^{21}\right\} \subset\{\Re \lambda>c\}, \\
& \text { (b) } \operatorname{spec}\left\{i \overline{\mathbb{A}}^{11}(z, q, \xi)+\overline{\mathbb{A}}^{12}\left(\bar{B}^{22}\right)^{-1} \overline{\mathbb{A}}^{21}+\bar{E}^{11}(z, q)\right\} \subset\{\Re \lambda>c\}, \\
& \text { (c) } \operatorname{spec}\{i \overline{\mathbb{A}}(z, q, \xi)+\bar{B}(z, q, \xi)+\bar{E}(z, q)\} \subset\{\Re \lambda>c\}
\end{aligned}
$$

for some constant $c>0$ which may vary from line to line.

Proof. - Hypothesis (H5) and Corollary 3.9 imply that (3.23)(a) holds for $|\xi|$ large and $|z| \geqslant Z$. Since $\overline{\mathbb{A}}^{11}(z, q, \xi)=\bar{A}^{11}(z, q, \xi)$ and

$$
\left|\overline{\mathbb{A}}^{12}\left(\bar{B}^{22}\right)^{-1} \overline{\mathbb{A}}^{21}-\bar{A}^{12}\left(\bar{B}^{22}\right)^{-1} \bar{A}^{21}\right|+\left|\bar{E}^{11}\right| \leqslant C e^{-\theta|z|}
$$

for some $\delta>0,(3.23)$ (a) is equivalent to (3.23)(b) for $|z| \geqslant Z_{1}$ large enough. The equivalence of (3.23)(b) and (3.23)(c) then follows from part (a) of Proposition 3.8.

Example 3.11. - Assume $s=N-1$ or, more generally, that

$$
\overline{\mathcal{L}}^{11}=\left(i \tau+\gamma+\sum_{j=1}^{d} \bar{a}_{j}(z, q) i \xi_{j}+\bar{e}\right) I
$$

with $a_{j}$, e scalar. Then (3.23) holds for $|z|$ and $|\xi|$ large if and only if

$$
\operatorname{spec}\left(\bar{A}^{12}\left(\bar{B}^{22}\right)^{-1} \bar{A}^{21}\right) \subset\{\Re \lambda>c\} .
$$

By (H3) the eigenvalues of $\bar{A}^{11}(z, q, \xi)$ are real and semisimple with constant multiplicities, and we can push further the diagonalization process. Denote by $\lambda_{k}(z, q, \xi)$ the distinct eigenvalues of $\bar{A}^{11}(z, q, \xi)=\overline{\mathbb{A}}^{11}(z, q, \xi)$ with multiplicities $r_{k}$. Then, locally in $\xi$, there exists a smooth, homogeneous symbol $V_{1}(z, q, \xi)$ of degree zero in $\xi$ such that

$$
\left(V_{1}\right)^{-1} \bar{A}^{11} V_{1}=\operatorname{diag}\left(\lambda_{k} I_{r_{k}}\right),
$$

where $I_{r}$ denotes the $r \times r$ identity matrix (see Remark 3.13). By perturbation there is, for $|\xi|$ large, a matrix $W_{1}=V_{1}+\mathrm{O}\left(|\xi|^{-1}\right)$ such that for $\widehat{A}^{11}$ as in (3.21) we have

$$
\left(W_{1}\right)^{-1} \widehat{A}^{11} W_{1}=\operatorname{diag}\left(\widehat{A}_{k}^{11}\right), \quad \widehat{A}_{k}^{11}=i \lambda_{k} I_{r_{k}}+C_{k}^{11},
$$

where the $r_{k} \times r_{k}$ blocks $C_{k}^{11}(z, q, \xi)$ are symbols of order zero in $\xi$. This yields the following additional corollary of Proposition 3.8:

COROLlaRY 3.12 (E). - Assume (H2), (H5), and (3.19). There exist positive constants $R, c$, and $Z_{1}$ such that for $|\xi| \geqslant R$ and $|z| \geqslant Z_{1}$, the following property, equivalent to those in (3.23), holds:

4 SÉRIE - TOME $39-2006-\mathrm{N}^{\circ} 1$ 


$$
\operatorname{spec} C_{k}^{11} \subset\{\Re \lambda>c\} \quad \text { for all } k,
$$

with $C_{k}^{11}$ as in (3.28).

Remark 3.13. - The results of Section 3.2.2 still hold for $W(z, q)$ satisfying only conditions (a) and (b) of Definition 2.16. This observation is used later in estimating solutions supported away from the front, where we must allow $u^{\prime}$ to be large.

\subsubsection{Reduction to a first-order system}

Separating the equation

$$
\mathcal{L}\left(z, q, \zeta, \partial_{z}\right) u=f
$$

into its first and second components and using

$$
\mathbb{A}_{j}^{1 k}=\widetilde{A}_{j}^{1 k}, \quad k=1,2,
$$

we obtain

(a) $\widetilde{A}_{d}^{11} \partial_{z} u^{1}+\widetilde{A}_{d}^{12} \partial_{z} u^{2}+E^{11} u^{1}+E^{12} u^{2}+A_{0}^{11}(i \tau+\gamma) u^{1}$

$$
+\sum_{j=1}^{d-1}\left(\widetilde{A}_{j}^{11} i \eta_{j} u^{1}+\widetilde{A}_{j}^{12} i \eta_{j} u^{2}\right)=f^{1}
$$

(b) $-\widetilde{B}_{d, d}^{22} \partial_{z}^{2} u^{2}+\mathbb{A}_{d}^{21} \partial_{z} u^{1}+\left(\mathbb{A}_{d}^{22}-\sum_{j=1}^{d-1}\left(\widetilde{B}_{j, d}^{22}+\widetilde{B}_{d, j}^{22}\right) i \eta_{j}\right) \partial_{z} u^{2}$

$$
+E^{21} u^{1}+E^{22} u^{2}+A_{0}^{21}(i \tau+\gamma) u^{1}+A_{0}^{22}(i \tau+\gamma) u^{2}
$$

$$
+\sum_{j=1}^{d-1} \mathbb{A}_{j}^{21} i \eta_{j} u^{1}+\left(\sum_{j=1}^{d-1} \mathbb{A}_{j}^{22} i \eta_{j}+\sum_{j, k=1}^{d-1} \widetilde{B}_{j, k}^{22} \eta_{j} \eta_{k}\right) u^{2}=f^{2} .
$$

Let

$$
U=\left(\begin{array}{c}
u^{1} \\
u^{2} \\
\partial_{z} u^{2}
\end{array}\right) \quad \text { and } \quad \mathcal{J} f=\left(\begin{array}{c}
\left(\widetilde{A}_{d}^{11}\right)^{-1} f^{1} \\
0 \\
-\left(\widetilde{B}_{d, d}^{22}\right)^{-1}\left(f^{2}-\mathbb{A}_{d}^{21}\left(\widetilde{A}_{d}^{11}\right)^{-1} f^{1}\right)
\end{array}\right)
$$

We can now rewrite the second-order $N \times N$ transmission problem (3.8) as an equivalent firstorder $(N+s) \times(N+s)$ transmission problem:

$$
\begin{aligned}
& \partial_{z} U-G(z, q, \zeta) U=\mathcal{J} f, \\
& {[U]=0 \quad \text { on } z=0,}
\end{aligned}
$$

where

$$
G=\left(\begin{array}{ccc}
G^{11} & G^{12} & G^{13} \\
0 & 0 & I \\
G^{31} & G^{32} & G^{33}
\end{array}\right)
$$

with

$$
\begin{aligned}
G^{11}(z, q, \zeta) & =-\left(\widetilde{A}_{d}^{11}\right)^{-1}(z, q)\left(E^{11}(z, q)+A_{0}^{11}(i \tau+\gamma)+\sum_{j=1}^{d-1} \widetilde{A}_{j}^{11} i \eta_{j}\right) \\
G^{12} & =-\left(\widetilde{A}_{d}^{11}\right)^{-1}\left(E^{12}+\sum_{j=1}^{d-1} \widetilde{A}_{j}^{12} i \eta_{j}\right)
\end{aligned}
$$




$$
\begin{aligned}
& G^{13}=-\left(\widetilde{A}_{d}^{11}\right)^{-1} \widetilde{A}_{d}^{12}, \\
& G^{31}=\left(\widetilde{B}_{d, d}^{22}\right)^{-1}\left(E^{21}+A_{0}^{21}(i \tau+\gamma)+\sum_{j=1}^{d-1} \mathbb{A}_{j}^{21} i \eta_{j}+\mathbb{A}_{d}^{21} G^{11}\right) \\
& G^{32}=\left(\widetilde{B}_{d, d}^{22}\right)^{-1}\left(E^{22}+A_{0}^{22}(i \tau+\gamma)+\sum_{j=1}^{d-1} \mathbb{A}_{j}^{22} i \eta_{j}+\sum_{j, k=1}^{d-1} \widetilde{B}_{j, k}^{22} \eta_{j} \eta_{k}\right. \\
&\left.\quad+\mathbb{A}_{d}^{21} G^{12}\right), \\
& G^{33}=\left(\widetilde{B}_{d, d}^{22}\right)^{-1}\left(\mathbb{A}_{d}^{22}-\sum_{j=1}^{d-1}\left(\widetilde{B}_{j, d}^{22}+\widetilde{B}_{d, j}^{22}\right) i \eta_{j}+\mathbb{A}_{d}^{21} G^{13}\right) .
\end{aligned}
$$

We note that:

$$
\begin{aligned}
& G^{11} \text { is first order in } \zeta, \\
& G^{12} \text { is first order in } \eta, \\
& G^{13} \text { is independent of } \zeta, \\
& G^{31} \text { is first order in } \zeta, \\
& G^{32} \text { is first order in } i \tau+\gamma \text { and second order in } \eta, \\
& G^{33} \text { is first order in } \eta .
\end{aligned}
$$

\subsubsection{Decoupling}

In the proof of the estimate (3.9), a key step is to obtain a decoupling of $G$ into hyperbolic and parabolic blocks. We will see in Section 3.2.7 that this can be done without any further change of dependent variable for frequencies in any region of the form $\gamma \geqslant C|\zeta|$. In this section we concentrate on the region $0 \leqslant \gamma \leqslant \delta|\zeta|$, where $\delta$ is some small enough constant to be chosen.

For later error control in the variable coefficient estimates, it is desirable to accomplish the decoupling with a conjugator whose entries are homogeneous symbols (like $|\zeta|$ ). After decoupling, the estimate for the hyperbolic block will be carried out using homogeneous symbols, but for the parabolic block we introduce a rescaling based on the parabolic symbol $\Lambda(\zeta)$ to obtain an optimal estimate.

The decoupling is easier when $G$ is rescaled as follows. If $U=\left(u^{1}, u^{2}, u^{3}\right)$ in (3.34) is replaced by $\widetilde{U}=\left(u^{1},|\zeta| u^{2}, u^{3}\right)$, then $G$ must be replaced by

$$
\widetilde{G}=\left(\begin{array}{ccc}
G^{11} & G^{12}|\zeta|^{-1} & G^{13} \\
0 & 0 & |\zeta| I_{s} \\
G^{31} & G^{32}|\zeta|^{-1} & G^{33}
\end{array}\right):=\left(\begin{array}{cc}
G^{11} & M^{12} \\
M^{21} & M^{22}
\end{array}\right),
$$

a zero-order perturbation of the first-order matrix $\left(\begin{array}{cc}G^{11} & 0 \\ M^{21} & M^{22}\end{array}\right)$. Note that $M^{22}$ and

$$
M_{i n}^{22}=\left(\begin{array}{cc}
0 & I \\
G^{32} & G^{33}
\end{array}\right)
$$

have the same eigenvalues.

Lemma $3.14(\mathrm{P}) .-$ Assume $(\mathrm{H} 2),(\mathrm{H} 3),(\mathrm{H} 8)$, and (3.19). There exist positive constants $c$, $\delta$, and $R$ such that for $|\zeta| \geqslant R, 0 \leqslant \gamma \leqslant \delta|\zeta|$ and all $z$, the distance between the spectrum of $G^{11}(z, q, \zeta)$ and the spectrum of $M^{22}(z, q, \zeta)$ is larger than $c|\zeta|$.

$4^{e}$ SÉRIE - TOME $39-2006-\mathrm{N}^{\circ} 1$ 
Proof. - The dependence on $(z, q)$ enters only through $W(z, q)$ and $\nu\left(h+h^{\prime}\right)$, so we may reduce by compactness to considering a single choice of $(z, q)$. Since we are concerned only with large $|\zeta|$, it suffices to consider just the principal terms (homogeneous of degree one in $\zeta$ ) in these matrices, and to show that the principal parts $G_{p}^{11}$ and $M_{p}^{22}$ have no common eigenvalue when $|\zeta|=1$ and $\gamma$ is small enough.

Suppose $\gamma=0$. Then by Proposition 2.10 the eigenvalues of $G_{p}^{11}$ are purely imaginary. If $\eta \neq 0$, (H2) implies that eigenvalues $\mu_{2}$ of $M_{p}^{22}$ satisfy $\Re \mu_{2} \neq 0$. Moreover, when $\eta=0$, we have $M_{p}^{22}=0$ and

$$
G_{p}^{11}=-\left(\widetilde{A}_{d}^{11}\right)^{-1} A_{0}^{11} i \tau
$$

has nonzero eigenvalues. Thus, for $\gamma=0$ and $|\zeta|=1$, the matrices $G_{p}^{11}$ and $M_{p}^{22}$ have no common eigenvalue. This remains true for $\gamma$ small.

Definition 3.15. - Let $\zeta=(\tau, \gamma, \eta)$ and for a multi-index $\alpha=\left(\alpha_{\tau}, \alpha_{\eta}\right)$, set $|\alpha|=\alpha_{\tau}+\left|\alpha_{\eta}\right|$ and $\|\alpha\|=2 \alpha_{\tau}+\left|\alpha_{\eta}\right|$.

Denote by $\Gamma^{m}$ the space of homogeneous symbols of order $m$

$$
\Gamma^{m}=\left\{h(z, q, \zeta) \in C^{\infty}:\left|\partial_{z, q}^{\beta} \partial_{\tau, \eta}^{\alpha} h\right| \leqslant C_{\beta \alpha}|\zeta|^{m-|\alpha|},|\zeta| \geqslant 1, \text { any } \alpha, \beta\right\} .
$$

Denote by $P \Gamma^{m}$ the space of parabolic symbols of order $m$

$$
P \Gamma^{m}=\left\{h(z, q, \zeta) \in C^{\infty}:\left|\partial_{z, q}^{\beta} \partial_{\tau, \eta}^{\alpha} h\right| \leqslant C_{\beta \alpha}|\Lambda(\zeta)|^{m-\|\alpha\|},|\zeta| \geqslant 1 \text {, any } \alpha, \beta\right\} .
$$

We use the same notation for spaces of homogeneous or parabolic matrix symbols of any fixed dimension.

The following corollary of Lemma 3.14 gives a partial decoupling of $\widetilde{G}$.

COROLLARY 3.16 (P). - Under the assumptions of Lemma 3.14, there is a homogeneous symbol

$$
W(z, q, \zeta)=\left(\begin{array}{cc}
I_{N-s} & 0 \\
W^{21} & I_{2 s}
\end{array}\right) \in \Gamma^{0}
$$

such that

$$
W^{-1} \widetilde{G} W=\left(\begin{array}{cc}
G^{11}+M^{12} W^{21} & M^{12} \\
-W^{21} M^{12} W^{21} & M^{22}-W^{21} M^{12}
\end{array}\right):=\widetilde{G}_{0} \in \Gamma^{1},
$$

where the off-diagonal blocks belong to $\Gamma^{0}$.

Proof. - A short computation using the explicit forms of $W, \widetilde{G}$, and $\widetilde{G}_{0}$ shows that we just need to choose $W^{21}$ satisfying

$$
W^{21} G^{11}-M^{22} W^{21}=M^{21} .
$$

Now $W^{21}$ is a $2 s \times(N-s)$ matrix, so we can identify it with an element of $\mathbb{C}^{p}$, where $p=2 s(N-s)$. Let

$$
h: \mathbb{C}^{p} \rightarrow \mathbb{C}^{p}
$$

be the linear map defined by the left side of (3.42) using this identification. The eigenvalues of $h$ are differences $\mu_{1}-\mu_{2}$, where $\mu_{1}$ (respectively, $\mu_{2}$ ) is an eigenvalue of $G^{11}$ (respectively, $M^{22}$ ). The map $h$ is given by a $p \times p$ matrix whose entries belong to $\Gamma^{1}$ and whose determinant satisfies

$$
|\operatorname{det} h| \geqslant C|\zeta|^{p}
$$


by Lemma 3.14. Thus, $h^{-1} \in \Gamma^{-1}$ and

$$
W^{21}=h^{-1}\left(M^{21}\right) \in \Gamma^{0} .
$$

From Corollary 3.16 we deduce readily the following partial decoupling of $G$ itself.

COROLLARY 3.17 (P). - Under the assumptions of Lemma 3.14 and for $W^{21}$ as in (3.40), let

$$
W^{21}=\left(\begin{array}{c}
W_{a}^{21} \\
W_{b}^{21}
\end{array}\right) \quad \text { and } \quad \mathcal{W}^{21}=\left(\begin{array}{c}
W_{a}^{21}|\zeta|^{-1} \\
W_{b}^{21}
\end{array}\right),
$$

where the $a$ and $b$ entries are each of size $s \times(N-s)$. Then

$$
\mathcal{W}=\left(\begin{array}{cc}
I & 0 \\
\mathcal{W}^{21} & I
\end{array}\right) \in \Gamma^{0}
$$

satisfies

$$
\mathcal{W}^{-1} G \mathcal{W}=\left(\begin{array}{ccc}
G^{11}+M^{12} W^{21} & G^{12} & G^{13} \\
G_{0}^{21} & -W_{a}^{21}|\zeta|^{-1} G^{12} & I_{s}-W_{a}^{21}|\zeta|^{-1} G^{13} \\
G_{0}^{31} & G^{32}-W_{b}^{21} G^{12} & G^{33}-W_{b}^{21} G^{13}
\end{array}\right):=G_{0}
$$

where

$$
\begin{aligned}
& G_{0}^{21}=-W_{a}^{21}\left(|\zeta|^{-2} G^{12} W_{a}^{21}+|\zeta|^{-1} G^{13} W_{b}^{21}\right) \in \Gamma^{-1}, \\
& G_{0}^{31}=-W_{b}^{21}\left(|\zeta|^{-1} G^{12} W_{a}^{21}+G^{13} W_{b}^{21}\right) \in \Gamma^{0} .
\end{aligned}
$$

Proof. - For each $(i, j)$ the equation expressing equality of the $(i, j)$ entries in $G \mathcal{W}=\mathcal{W} G_{0}$ is a multiple of the corresponding equation for $\widetilde{G} W=W \widetilde{G}_{0}$.

Remark 3.18. - We will use the partial decoupling given by Corollary 3.17 in the high frequency estimate. The complete decoupling of $\widetilde{G}$ given in the next proposition is useful for the spectral analysis of the block

$$
G^{11}+M^{12} W^{21}=G_{0}^{11} .
$$

Proposition 3.19 (P).- Under the assumptions of Lemma 3.14, there is a matrix $\mathbb{W}(z, q, \zeta) \in \Gamma^{0}$ such that

$$
\mathbb{W}^{-1} \widetilde{G} \mathbb{W}=\left(\begin{array}{cc}
\widehat{G}^{11} & 0 \\
0 & \widehat{M}^{22}
\end{array}\right):=\widehat{G}
$$

where

$$
\begin{aligned}
\widehat{G}^{11} & =G^{11}+M^{12} W^{21}=G_{0}^{11} \in \Gamma^{1}, \\
\widehat{M}^{22} & =M^{22}+g \in \Gamma^{1}, \quad g \in \Gamma^{0} .
\end{aligned}
$$

Proof. - Set

$$
\mathbb{W}=\left(\begin{array}{cc}
I_{N-s} & b \\
W^{21} & I_{2 s}
\end{array}\right)
$$

where $b \in \Gamma^{-1}$, and solve for $b$ and $g$ by equating corresponding entries in

$$
\widetilde{G} \mathbb{W}=\mathbb{W} \widehat{G} \text {. }
$$

Define $g$ in terms of $b$ using equality of the $(2,2)$ entries, substitute into the equality for the $(1,2)$ entries, and solve the resulting nonlinear equation for $b$ using invertibility of

$$
\tilde{h}(b)=G^{11} b-b M^{22},
$$

as follows from Lemma 3.14.

$4^{\mathrm{e}}$ SÉRIE - TOME $39-2006-\mathrm{N}^{\circ} 1$ 


\subsubsection{Spectral properties of $\widehat{G}^{11}$}

Let $\mu_{k}(z, q, \zeta)$ of multiplicity $s_{k}, k=1, \ldots, k_{0}$, denote the distinct eigenvalues of $G_{p}^{11}(z, q, \zeta)$, the principal part of $G^{11}$. By Proposition 2.10 the $\mu_{k}$ are purely imaginary and semisimple with constant multiplicity for $\gamma=0$. In addition we have:

Proposition 3.20 (P). - Assume (H3), (H8), and (3.19). There exists a $\delta>0$ such that for $0 \leqslant \gamma \leqslant \delta|\zeta|$, the $\mu_{k}$ are $C^{\infty}$ in $(z, q, \eta)$, analytic in $\tau-i \gamma$ and semisimple with constant multiplicity. Moreover, for $\gamma>0, \Re \mu_{k} \neq 0$ and has the same sign for all $k$, namely $-\operatorname{sgn}(\beta)$, where $\beta$ is any eigenvalue of $\bar{A}_{d}^{11}(z, q)$.

Proof. - The $\mu_{k}(z, q, \cdot)$ are real analytic in $(\tau, \eta) \in \mathbb{R}^{d} \backslash 0$ and homogeneous of degree one, so can be extended analytically in $\tau-i \gamma$ near $\gamma=0$ as smooth functions of $(z, q, \eta)$. The eigenvectors associated to $\mu_{k}$ extend analytically as well. Semisimplicity and constant multiplicity of the extended $\mu_{k}$ follow from analyticity.

Hyperbolicity of $\bar{A}^{11}(z, q, \xi)$ (H3) implies $\Re \mu_{k} \neq 0$ for $\gamma>0$. Setting $(\tau, \eta)=0$ in $G_{p}^{11}$ and using (H8) shows that the signs are as described.

Using Proposition 3.20 and arguing as in (3.28), we obtain, for $|\zeta|$ large and $0 \leqslant \gamma \leqslant \delta|\zeta|$, matrices $\mathcal{V}(z, q, \zeta) \in \Gamma^{0}$ such that

$$
\mathcal{V}^{-1} \widehat{G}^{11} \mathcal{V}=\operatorname{diag}\left(\mu_{k} I_{s_{k}}+R_{k}\right)
$$

where $R_{k} \in \Gamma^{0}$ is an $s_{k} \times s_{k}$ block, $k=1, \ldots, k_{0}$.

Remark 3.21. - At first sight the matrices $\mathcal{V}(z, q, \zeta)$ and $R_{k}$ can be chosen smoothly only locally in $(z, q, \zeta)$. A classical result of Kato [20, pp. 99-102] implies that for $(q, \zeta)$ with $|\zeta|$ large and lying in a conic neighborhood of an arbitrary basepoint $(q, \hat{\zeta})$ such that $0 \leqslant \hat{\gamma} \leqslant \delta$, such conjugators can be chosen smoothly in $(z, q, \zeta)$ for all $z$. Here $\hat{\zeta}=\zeta /|\zeta|$. We use this observation later in the variable coefficient analysis.

The main result of this subsection, needed for the estimate of the hyperbolic block, is that for all $k$, the real part of spec $R_{k}$ is bounded away from zero and has the same fixed sign, namely $-\operatorname{sgn}(\beta)=\operatorname{sgn}\left(\Re \mu_{k}\right)$, where $\beta$ is any eigenvalue of $\bar{A}_{d}^{11}(z, q)$ (recall (H8)). Recalling that $(z, q)$ dependence enters only through $W(z, q)$, we let

$$
R_{k}^{ \pm \infty}(q, \zeta)=\lim _{z \rightarrow \pm \infty} R_{k}(z, q, \zeta)
$$

Proposition 3.22. - Assume (H2), (H3), (H5), (H8), and (3.19). Let $\mu_{k}(z, q, \zeta)$ denote the distinct eigenvalues of $G_{p}^{11}$, the principal part of $G^{11}$, and let $\beta$ be any eigenvalue of $\bar{A}_{d}^{11}$. Let $\nu_{k l}$ denote the eigenvalues of $R_{k}$. There exist positive constants $R, Z_{1}, c$, and $\delta$ such that:

(i) (P) $(-\operatorname{sgn} \beta) \Re \mu_{k} \geqslant c \gamma$ for $0 \leqslant \gamma \leqslant \delta|\zeta|$, all $(z, q)$, and all $k$.

(ii) (E) For $|\zeta| \geqslant R,|z| \geqslant Z_{1}$, and $0 \leqslant \gamma \leqslant \delta|\zeta|$, we have

$$
\begin{aligned}
& \operatorname{sgn}\left(\Re \nu_{k l}\right)=\operatorname{sgn}\left(\Re \mu_{k}\right)=-\operatorname{sgn}(\beta) \quad \text { for all } k, l, \\
& \left|\Re \nu_{k l}\right| \geqslant c \quad \text { for all } k, l .
\end{aligned}
$$

The proof is given below.

In view of (3.58), after modifying $\mathcal{V}$ if necessary, we may assume for $\zeta$ as in (3.58)

$$
-\operatorname{sgn}(\beta) \Re R_{k} \geqslant c>0 \text { for }|z| \geqslant Z_{1} \text { and all } k .
$$

Together with part (i) of the proposition, this gives 
Corollary $3.23(\mathrm{P})$. - With assumptions as in Proposition 3.22, we have for all $k,|\zeta| \geqslant R$, $0 \leqslant \gamma \leqslant \delta|\zeta|$, and all $(z, q)$,

$$
\text { (P) } \quad-\operatorname{sgn}(\beta) \Re\left(\mu_{k}(z, q, \zeta) I_{s_{k}}+R_{k}^{ \pm \infty}(q, \zeta)\right) \geqslant c(\gamma+1) .
$$

Remark 3.24. - The proof of Proposition 3.22 depends on the next lemma. In preparation note first that for $|\zeta|$ large and $\delta$ small, the functions $\widehat{G}^{11}$ and $R_{k}$ in (3.56) are analytic in $\tau-i \gamma$ for $0 \leqslant \gamma \leqslant \delta|\zeta|$. Writing $\lambda_{k}(z, q, \xi)=\lambda_{k}\left(z, q, \eta, \xi_{d}\right)$ for $\lambda_{k}$ as in (3.28) and letting $\xi_{d}$ denote a complex variable now, we see by arguing as in Proposition 3.20 that the $\lambda_{k}$ can be extended analytically in $\xi_{d}$ to $0 \leqslant\left|\Im \xi_{d}\right| \leqslant \delta_{1}\left|\eta, \xi_{d}\right|$ for $\delta_{1}$ small enough, as semisimple eigenvalues of $\bar{A}^{11}\left(z, q, \eta, \xi_{d}\right)$ with constant multiplicities. Moreover, for large $\left|\eta, \xi_{d}\right|$ the functions $\widehat{A}^{11}$ and $C_{k}^{11}$ in (3.28) extend analytically in $\xi_{d}$ to $0 \leqslant\left|\Im \xi_{d}\right| \leqslant \delta_{1}\left|\eta, \xi_{d}\right|$.

Lemma $3.25(\mathrm{P})$. - Assume (H2), (H3), (H8), and (3.19). Fix $C_{1}>0$ and assume $|i \tau+i \gamma| \leqslant$ $C_{1}\left|\eta, \xi_{d}\right|^{2}$. There exist positive constants $R$ and $\delta$ such that for $\left|\eta, \xi_{d}\right| \geqslant R, 0 \leqslant \gamma \leqslant \delta|\zeta|$, and $\left|\Im \xi_{d}\right| \leqslant \delta\left|\eta, \xi_{d}\right|$ we have

$$
\operatorname{det}\left(i \xi_{d}-\widehat{G}^{11}(z, q, \zeta)\right)=c\left(z, q, \zeta, \xi_{d}\right) \operatorname{det}\left(i \tau+\gamma+\widehat{A}^{11}\left(z, q, \eta, \xi_{d}\right)\right)
$$

where

$$
c\left(z, q, \zeta, \xi_{d}\right)=\left(\operatorname{det} \bar{A}_{d}^{11}\right)^{-1}+\mathrm{O}\left(\left|\zeta, \xi_{d}\right|^{-1}\right) .
$$

Proof. - 1. With $\overline{\mathcal{L}}$ as in (3.16) we have

$$
\begin{aligned}
& \operatorname{det}\left(i \xi_{d}-\widetilde{G}(z, q, \zeta)\right)=\operatorname{det}\left(i \xi_{d}-G\right)=c_{1}(z, q) \operatorname{det} \overline{\mathcal{L}}, \quad \text { with } \\
& c_{1}(z, q)=(-1)^{s} \operatorname{det}\left(\widetilde{A}_{d}^{11}\right)^{-1} \operatorname{det}\left(\widetilde{B}_{d, d}^{22}\right)^{-1} \operatorname{det} A_{0},
\end{aligned}
$$

as follows by performing obvious row and column operations on $\operatorname{det}\left(i \xi_{d}-G\right)$. Propositions 3.8 and 3.19 thus give

$$
\operatorname{det}\left(i \xi_{d}-\widehat{G}^{11}\right) \operatorname{det}\left(i \xi_{d}-\widehat{M}^{22}\right)=c_{1}(z, q) \operatorname{det}\left(i \tau+\gamma+\widehat{A}^{11}\right) \operatorname{det}\left(i \tau+\gamma+\widehat{B}^{22}\right) .
$$

2. We would like to cancel the final factors on each side of (3.64). First, with $M_{i n}^{22}$ as in (3.38) we have

$$
\begin{aligned}
\operatorname{det}\left(i \xi_{d}-\widehat{M}^{22}\right) & =\operatorname{det}\left(i \xi_{d}-M^{22}\right)+\mathrm{O}\left(\left|\zeta, \xi_{d}\right|^{2 s-1}\right) \\
& =\operatorname{det}\left(i \xi_{d}-M_{i n}^{22}\right)+\mathrm{O}\left(\left|\zeta, \xi_{d}\right|^{2 s-1}\right) \\
& =c_{2}(z, q) \operatorname{det}\left(i \tau+\gamma+\bar{B}^{22}(z, q, \xi)\right)+\mathrm{O}\left(\left|\zeta, \xi_{d}\right|^{2 s-1}\right) \\
& =c_{2}(z, q) \operatorname{det}\left(i \tau+\gamma+\widehat{B}^{22}(z, q, \xi)\right)+\mathrm{O}\left(\left|\zeta, \xi_{d}\right|^{2 s-1}\right)
\end{aligned}
$$

where $c_{2}(z, q)=(-1)^{s} \operatorname{det}\left(\widetilde{B}_{d, d}^{22}\right)^{-1} \operatorname{det} A_{0}^{22}$, as follows by performing row operations on $\operatorname{det}\left(i \xi_{d}-M_{i n}^{22}\right)$.

3. By (H2), for $\xi=\left(\eta, \xi_{d}\right)$ real and $\mu$ an eigenvalue of $\bar{B}^{22}(z, q, \xi)$ we have

$$
\Re \mu \geqslant|\xi|^{2} .
$$

For $\delta$ small (3.66) continues to hold for $0 \leqslant\left|\Im \xi_{d}\right| \leqslant \delta\left|\eta, \xi_{d}\right|$. Thus, for $0 \leqslant\left|\Im \xi_{d}\right| \leqslant \delta\left|\eta, \xi_{d}\right|$, and $|i \tau+\gamma| \leqslant C_{1}\left|\eta, \xi_{d}\right|^{2}$, there holds

$$
\left|\operatorname{det}\left(i \tau+\gamma+\bar{B}^{22}(z, q, \xi)\right)\right| \approx\left|\eta, \xi_{d}\right|^{2 s} .
$$

$4^{e}$ SÉRIE - TOME $39-2006-\mathrm{N}^{\circ} 1$ 
We may now cancel in (3.64) and obtain (3.61) with

$$
c\left(z, q, \zeta, \xi_{d}\right)=\frac{c_{1}(z, q)}{c_{2}(z, q)}+\mathrm{O}\left(\left|\zeta, \xi_{d}\right|^{-1}\right)=\left(\operatorname{det} \bar{A}_{d}^{11}\right)^{-1}+\mathrm{O}\left(\left|\zeta, \xi_{d}\right|^{-1}\right)
$$

Before proving Proposition 3.22, we first illustrate it in an important special case that includes the Navier-Stokes equations.

Example 3.26. - Assume $s=N-1$, so that

$$
\overline{\mathcal{L}}^{11}=i \tau+\gamma+\sum_{j=1}^{d} \bar{A}_{j}^{11}(z, q) i \xi_{j}+\bar{E}^{11}
$$

with $\bar{A}^{11}, \bar{E}^{11}$ scalar. Writing

$$
\begin{aligned}
& \widehat{A}^{11}(z, q, \xi)=i \bar{A}^{11}(z, q, \xi)+\bar{E}^{11}(z, q)+\mathcal{R}(z, q, \xi), \\
& \widehat{G}^{11}(z, q, \zeta)=G^{11}(z, q, \zeta)+R_{1}(z, q, \zeta)
\end{aligned}
$$

we have $\Re \mu_{1}=-\left(\bar{A}_{d}^{11}\right)^{-1} \gamma$ now and, by Corollary 3.12,

$$
\mathcal{R}(z, q, \xi) \geqslant c>0 \text { for }|\xi| \text { and }|z| \text { large. }
$$

Eq. (3.61) is

$$
i \xi_{d}-\left(G^{11}+R_{1}\right)=c\left(z, q, \zeta, \xi_{d}\right)\left(i \tau+\gamma+\sum_{j=1}^{d-1} \bar{A}_{j}^{11} i \eta_{j}+\bar{A}_{d}^{11} i \xi_{d}+\bar{E}^{11}+\mathcal{R}\right) .
$$

Using (3.62) and setting

$$
\tilde{\xi}_{d}=-\left(\bar{A}_{d}^{11}\right)^{-1}\left(\tau-i \gamma+\sum_{j=1}^{d-1} \bar{A}_{j}^{11} \eta_{j}-i \bar{E}^{11}\right)
$$

we find

$$
R_{1}(z, q, \zeta)=-\left.\left(\bar{A}_{d}^{11}\right)^{-1} \mathcal{R}\left(z, q, \eta, \xi_{d}\right)\right|_{\xi_{d}=\tilde{\xi}_{d}}+\mathrm{O}\left(|\zeta|^{-1}\right) .
$$

With (3.71) this implies the conclusions of Proposition 3.22 for this example. In particular, $\operatorname{sgn} \Re R_{1}=-\operatorname{sgn} \beta=-\operatorname{sgn} \bar{A}_{d}^{11}$ and $\left|\Re R_{1}\right| \geqslant c>0$.

Proof of Proposition 3.22. - 1. If $i \xi_{d} \in \operatorname{spec} G_{p}^{11}(z, q, \zeta)$, then

$$
-(i \tau+\gamma) \in \operatorname{spec} i \bar{A}^{11}\left(z, q, \eta, \xi_{d}\right)=\operatorname{spec}\left(\sum_{j=1}^{d-1} \bar{A}_{j}^{11} i \eta_{j}+\bar{A}_{d}^{11} i \xi_{d}\right)
$$

so

$$
|i \tau+\gamma| \leqslant C\left|\eta, \xi_{d}\right| \quad \text { and } \quad\left|\eta, \xi_{d}\right| \approx|\zeta|
$$

for some $C>0$. Thus, (3.74) holds also for $i \xi_{d} \in \operatorname{spec} \widehat{G}^{11}(z, q, \zeta)$ for $|\zeta|$ large, so we may assume (3.74) in the proof of Proposition 3.22. In particular, Lemma 3.25 applies.

2. Introducing polar coordinates $\zeta=\rho \hat{\zeta}=\rho(\hat{\tau}, \hat{\gamma}, \hat{\eta})$ and setting

$$
\varepsilon=1 / \rho, \quad \hat{\xi}_{d}=\xi_{d} / \rho,
$$

we may use the block decompositions of $\widehat{A}^{11}$ (3.28) and $\widehat{G}^{11}$ (3.56) to rewrite Eq. (3.61) as 


$$
\begin{aligned}
& \prod_{k} \operatorname{det}\left(\left(i \hat{\xi}_{d}-\mu_{k}(z, q, \hat{\tau}-i \hat{\gamma}, \hat{\eta})\right) I_{s_{k}}-\varepsilon R_{k}(z, q, \hat{\tau}-i \hat{\gamma}, \hat{\eta}, \varepsilon)\right) \\
& \quad=c \prod_{j} \operatorname{det}\left(\left(i \hat{\tau}+\hat{\gamma}+i \lambda_{j}\left(z, q, \hat{\eta}, \hat{\xi}_{d}\right)\right) I_{r_{j}}+\varepsilon C_{j}^{11}\left(z, q, \hat{\eta}, \hat{\xi}_{d}, \varepsilon\right)\right) .
\end{aligned}
$$

Here

$$
\begin{aligned}
R_{k}(z, q, \hat{\tau}-i \hat{\gamma}, \hat{\eta}, \varepsilon) & :=R_{k}(z, q, \zeta)=R_{k 0}(z, q, \hat{\zeta})+\mathrm{O}(\varepsilon), \\
C_{j}^{11}\left(z, q, \hat{\eta}, \hat{\xi}_{d}, \varepsilon\right) & :=C_{j}^{11}\left(z, q, \eta, \xi_{d}\right)=C_{j 0}^{11}\left(z, q, \hat{\eta}, \hat{\xi}_{d}\right)+\mathrm{O}(\varepsilon),
\end{aligned}
$$

where $R_{k 0}$ (respectively $C_{j 0}^{11}$ ) is homogeneous of degree zero in $\zeta$ (respectively $\left(\eta, \xi_{d}\right)$ ).

3. We work near a basepoint $(\underline{\hat{\tau}}, 0, \underline{\hat{\eta}}) \neq 0, \underline{\varepsilon}=0$. Consider an eigenvalue (necessarily purely imaginary) $\underline{\hat{\mu}}_{k}=i \underline{\hat{\xi}}_{d}$ of $G_{p}^{11}(z, q, \hat{\underline{\tau}}, 0, \underline{\hat{\eta}})$. By (H3) there exists a unique eigenvalue $\lambda_{j}$ of $\bar{A}^{11}(z, q, \xi)$ such that

$$
\underline{\hat{\tau}}+\lambda\left(z, q, \underline{\hat{\eta}}, \underline{\hat{\xi}}_{d}\right)=0 .
$$

Moreover, by (2.32) $\left(\underline{\hat{\tau}}, \underline{\hat{\underline{\eta}}}, \underline{\hat{\xi}}_{d}\right)$ is nonglancing:

$$
\partial_{\xi_{d}} \lambda_{j}\left(z, q, \underline{\hat{\eta}}, \underline{\hat{\xi}}_{d}\right):=\alpha \in \mathbb{R} \backslash 0 .
$$

From (3.76) we obtain

$$
\begin{aligned}
\triangle_{j} & :=\operatorname{det}\left(\left(i \hat{\tau}+\hat{\gamma}+i \lambda_{j}\left(z, q, \hat{\eta}, \hat{\xi}_{d}\right)\right) I_{r_{j}}+\varepsilon C_{j}^{11}\left(z, q, \hat{\eta}, \hat{\xi}_{d}, \varepsilon\right)\right)=c^{\prime} \tilde{\triangle}_{k} \\
& :=c^{\prime} \operatorname{det}\left(\left(i \hat{\xi}_{d}-\mu_{k}(z, q, \hat{\tau}-i \hat{\gamma}, \hat{\eta})\right) I_{s_{k}}-\varepsilon R_{k}(z, q, \hat{\tau}-i \hat{\gamma}, \hat{\eta}, \varepsilon)\right),
\end{aligned}
$$

with $c^{\prime}\left(z, q, \hat{\zeta}, \hat{\xi}_{d}, \varepsilon\right) \neq 0$ near the basepoint.

4. Taylor expanding (3.80) about the basepoint with $\xi_{d}^{\prime}=\hat{\xi}_{d}-\hat{\xi}_{d}$ and $\tau^{\prime}=\hat{\tau}-i \hat{\gamma}-\underline{\hat{\tau}}$ both complex gives

$$
\begin{aligned}
& \triangle_{j}\left(z, q, \hat{\tau}-i \hat{\gamma}, \hat{\underline{\eta}}, \xi_{d}, \varepsilon\right) \\
& \quad=\operatorname{det}\left(i\left(\tau^{\prime}+\alpha \xi_{d}^{\prime}\right) I_{r_{j}}+\varepsilon \underline{C}_{j}^{11}\right)+\mathrm{O}\left(\left|\tau^{\prime}, \xi_{d}^{\prime}, \varepsilon\right|^{r_{j}+1}\right) \\
& \quad=\underline{c}^{\prime} \widetilde{\triangle}_{k}\left(z, q, \hat{\tau}-i \hat{\gamma}, \underline{\hat{\eta}}, \xi_{d}, \varepsilon\right) \\
& \quad=\underline{c}^{\prime} \operatorname{det}\left(i\left(\xi_{d}^{\prime}-\tau^{\prime} \partial_{\gamma} \Re \mu_{k}(z, q, \hat{\underline{\tau}}, \underline{\hat{\eta}})\right) I_{s_{k}}-\varepsilon \underline{R}_{k}\right)+\mathrm{O}\left(\left|\tau^{\prime}, \xi_{d}^{\prime}, \varepsilon\right|^{s_{k}+1}\right),
\end{aligned}
$$

where underlining indicates evaluation at the basepoint. Taking $\varepsilon=0$ and $\tau^{\prime}=-i \hat{\gamma}$ in (3.81) and equating leading terms gives

$$
i^{r_{j}}\left(-i \hat{\gamma}+\alpha \xi_{d}^{\prime}\right)^{r_{j}}=\underline{c}^{\prime} i^{s_{k}}\left(\xi_{d}^{\prime}+i \hat{\gamma} \partial_{\gamma} \Re \mu_{k}\right)^{s_{k}},
$$

from which we conclude

$$
r_{j}=s_{k} \quad \text { and } \quad \partial_{\gamma} \Re \mu_{k}(z, q, \underline{\hat{\tau}}, \underline{\hat{\eta}})=-\frac{1}{\alpha} \in \mathbb{R} \backslash 0,
$$

giving, in particular, part (i) of Proposition 3.22. (We knew $r_{j}=s_{k}$ already from part 3 of the proof of Proposition 2.10.)

5. Now take $\tau^{\prime}=0$ and equate leading terms in (3.81) to get

$$
\operatorname{det}\left(i \alpha \xi_{d}^{\prime} I_{r_{j}}+\varepsilon \underline{C}_{j}^{11}\right)=\underline{c}^{\prime} \operatorname{det}\left(i \xi_{d}^{\prime} I_{r_{j}}-\varepsilon \underline{R}_{k}\right) .
$$

Thus, the eigenvalues of $\underline{R}_{k}$ are the same as the eigenvalues of $-\frac{1}{\alpha} \underline{C}_{j}^{11}$. By Corollary 3.12 this implies that for $|z|$ large, if $\nu_{k l}$ is an eigenvalue of $\underline{R}_{k}(z, q, \hat{\underline{\tau}}, \underline{\hat{\eta}})$, then 


$$
\Re \nu_{k l} \neq 0 \quad \text { and } \quad \operatorname{sgn}\left(\Re \nu_{k l}\right)=\operatorname{sgn}\left(-\frac{1}{\alpha}\right)=\operatorname{sgn}\left(\partial_{\gamma} \Re \mu_{k}\right)=-\operatorname{sgn}(\beta) .
$$

The last equality is a consequence of Proposition 3.20. By continuity and compactness, (3.85) implies part (ii) of Proposition 3.22.

\subsubsection{Spectral properties of the parabolic block $M_{i n}^{22}$}

Introduce "parabolic polar coordinates at infinity"

$$
\begin{aligned}
& \Lambda_{0}(\zeta)=\left(\tau^{2}+\gamma^{2}+|\eta|^{4}\right)^{1 / 2}, \\
& \check{\zeta}=(\check{\tau}, \check{\gamma}, \check{\eta})=\left(\frac{\tau}{\Lambda_{0}^{2}}, \frac{\gamma}{\Lambda_{0}^{2}}, \frac{\eta}{\Lambda_{0}}\right),
\end{aligned}
$$

and write

$$
\begin{array}{ll}
G^{32}(z, q, \zeta)=\Lambda_{0}^{2} \check{G}^{32}, & G^{33}=\Lambda_{0} \check{G}^{33}, \\
M_{i n}^{22}=\left(\begin{array}{cc}
0 & I \\
G^{32} & G^{33}
\end{array}\right), \quad \check{M}_{i n}^{22}:=\left(\begin{array}{cc}
0 & I \\
\check{G}^{32} & \check{G}^{33}
\end{array}\right) .
\end{array}
$$

In the next proposition we allow $q$ to vary in a set $\mathcal{Q}^{*}$ which may be larger than $\mathcal{Q}=\mathcal{C} \times \mathcal{O}$ as in (3.19).

Proposition $3.27(\mathrm{P})$. - Assume $(\mathrm{H} 2)$ and that for all $(z, q) \in \mathbb{R} \times \mathcal{Q}^{*}, W(z, q) \in \mathcal{U}^{*}$. There exist positive constants $R$ and $C$ such that for $|\zeta| \geqslant R$ and all $(z, q)$, the $2 s \times 2 s$ matrix $M_{i n}^{22}$ (respectively $\check{M}_{i n}^{22}$ ) has s eigenvalues, counted with their multiplicities, in $\Re \mu>0$ and $s$ eigenvalues in $\Re \mu<0$. They satisfy $|\Re \mu| \geqslant C \Lambda_{0}(\zeta)$ (respectively $|\Re \mu| \geqslant C$ ).

Proof. - The proof is identical to that of Lemma 2.5(i) in [30]. Hypothesis (H2) and compactness imply the result for $\check{M}_{i n}^{22}$. The result for $M_{i n}^{22}$ follows since

$$
\operatorname{spec}\left(M_{i n}^{22}\right)=\operatorname{spec}\left(\Lambda_{0} \check{M}_{i n}^{22}\right) .
$$

The following immediate corollary of Proposition 3.27 will be used in the construction of symmetrizers for the parabolic block. Let $\lambda=1 / \Lambda_{0}$ and for any $z$ let $\Pi_{n}(z, q, \check{\zeta}, \lambda)$ (respectively $\Pi_{p}(z, q, \check{\zeta}, \lambda)$ denote the spectral projectors onto the $s$-dimensional invariant spaces $\mathbb{F}_{n}^{\infty}(z, q, \check{\zeta}, \lambda) \subset \mathbb{C}^{2 s}$ (respectively $\mathbb{F}_{p}^{\infty}(z, q, \breve{\zeta}, \lambda)$ ) of $\check{M}_{i n}^{22}$ associated to eigenvalues with negative (respectively positive) real part. Proposition 3.27 implies the projectors are defined and smooth for $\lambda \in\left[0, \lambda_{0}\right]$ for some small $\lambda_{0}$. Define

$$
\begin{gathered}
\Gamma: \mathbb{C}^{4 s} \rightarrow \mathbb{C}^{2 s}, \quad \Gamma\left(v_{+}, v_{-}\right):=v_{+}-v_{-}, \\
\mathcal{F}^{\infty}(z, q, \check{\zeta}, \lambda)=\mathbb{F}_{n}^{\infty}(z, q, \check{\zeta}, \lambda) \times \mathbb{F}_{p}^{\infty}(z, q, \check{\zeta}, \lambda) \subset \mathbb{C}^{4 s} .
\end{gathered}
$$

COROLlaRY 3.28 (P). - Under the assumptions of Proposition 3.27, there exists $\lambda_{0}>0$ such that the following two equivalent statements hold for $\lambda \in\left[0, \lambda_{0}\right]$ and all $(z, q)$ :

$$
\begin{gathered}
\mathbb{C}^{2 s}=\mathbb{F}_{n}^{\infty}(z, q, \check{\zeta}, \lambda) \oplus \mathbb{F}_{p}^{\infty}(z, q, \check{\zeta}, \lambda), \\
\mathcal{F}^{\infty}(z, q, \check{\zeta}, \lambda) \cap \operatorname{ker} \Gamma=\{0\} .
\end{gathered}
$$

Remark 3.29. - We use (3.90) only for $z=0$ in the construction of symmetrizers. In that case the statement implies that an appropriately rescaled Evans function (as in [12, Proposition 2.12]) is bounded away from zero for $|\zeta|$ large. The construction can be done without introducing such an Evans function, so we do not define it here. 


\subsubsection{Estimates}

In this subsection we complete the proof of Proposition 3.5, using the reduction to a first-order system (3.34). Let $f=\left(f_{1}, f_{2}\right)$ be as in the estimate (3.9).

Proof of Proposition 3.5. - 1. Reductions; the case $0 \leqslant \gamma \leqslant \delta|\zeta|$. For $U=\left(u_{1}, u_{2}, u_{3}\right)$ satisfying the transmission problem (3.34):

$$
\begin{gathered}
\partial_{z} U-G(z, q, \zeta) U=\mathcal{J} f:=\left(\begin{array}{c}
g_{1} \\
0 \\
g_{3}
\end{array}\right), \\
{[U]=0 \quad \text { on } z=0}
\end{gathered}
$$

to prove the estimate (3.9) it suffices to show

$$
\begin{aligned}
& (1+\gamma)\left\|u_{1}\right\|+\Lambda\left\|u_{2}\right\|+\left\|u_{3}\right\|+\sqrt{1+\gamma}\left|u_{1}(0)\right|+\Lambda^{1 / 2}\left|u_{2}(0)\right|+\Lambda^{-1 / 2}\left|u_{3}(0)\right| \\
& \quad \leqslant C\left(\left\|g_{1}\right\|+\Lambda^{-1}\left\|g_{3}\right\|\right)
\end{aligned}
$$

since $\left\|g_{1}\right\|+\Lambda^{-1}\left\|g_{3}\right\| \leqslant C\left(\left\|f_{1}\right\|+\Lambda^{-1}\left\|f_{2}\right\|\right)$.

We first treat the more difficult case where the frequency $\zeta$ satisfies $0 \leqslant \gamma \leqslant \delta|\zeta|$.

For the conjugator $\mathcal{W}$ as in (3.48), set $U=\mathcal{W} V$ to derive the equivalent problem for $V=\left(v_{1}, v_{2}, v_{3}\right)$ :

$$
\begin{gathered}
V_{z}-G_{0} V=\mathcal{W}^{-1}\left(\begin{array}{c}
g_{1} \\
0 \\
g_{3}
\end{array}\right)-\left(\mathcal{W}^{-1} \mathcal{W}_{z}\right) V=\left(\begin{array}{c}
g_{1} \\
\mathrm{O}\left(|\zeta|^{-1}\right) g_{1} \\
\mathrm{O}(1) g_{1}+g_{3}
\end{array}\right)+\left(\begin{array}{c}
0 \\
\mathrm{O}\left(|\zeta|^{-1}\right) v_{1} \\
\mathrm{O}(1) v_{1}
\end{array}\right), \\
{[V]=0 \quad \text { on } z=0}
\end{gathered}
$$

where

$$
G_{0}=\left(\begin{array}{ccc}
\widehat{G}^{11} & \mathrm{O}(|\eta|) & \mathrm{O}(1) \\
\mathrm{O}\left(|\zeta|^{-1}\right) & \mathrm{O}(1) & I+\mathrm{O}\left(|\zeta|^{-1}\right) \\
\mathrm{O}(1) & G^{32}+\mathrm{O}(|\eta|) & G^{33}+\mathrm{O}(1)
\end{array}\right) .
$$

Estimating the components of $U$ in terms of those of $V$, we reduce to proving the estimate

$$
\begin{aligned}
& (1+\gamma)\left\|v_{1}\right\|+\Lambda\left\|v_{2}\right\|+\left\|v_{3}\right\|+\sqrt{1+\gamma}\left|v_{1}(0)\right|+\Lambda^{1 / 2}\left|v_{2}(0)\right|+\Lambda^{-1 / 2}\left|v_{3}(0)\right| \\
& \quad \leqslant C\left(\left\|g_{1}\right\|+\Lambda^{-1}\left\|g_{3}\right\|\right)
\end{aligned}
$$

for (3.93).

Next use (3.94) to rearrange (3.93)

$$
\begin{aligned}
V_{z} & -\left(\begin{array}{ccc}
\widehat{G}^{11} & 0 & 0 \\
0 & 0 & I \\
0 & G^{32} & G^{33}
\end{array}\right) V \\
& =\left(\begin{array}{c}
\mathrm{O}\left(|\zeta|^{-1}\right) g_{1}+\mathrm{O}\left(|\zeta|^{-1}\right) v_{1}+\mathrm{O}(1) v_{2}+\mathrm{O}\left(|\zeta|^{-1}\right) v_{3} \\
\mathrm{O}(1) g_{1}+g_{3}+\mathrm{O}(1) v_{1}+\mathrm{O}(|\eta|) v_{2}+\mathrm{O}(1) v_{3}
\end{array}\right):=\left(\begin{array}{c}
h_{1} \\
h_{2} \\
h_{3}
\end{array}\right) .
\end{aligned}
$$

The estimate (3.95) follows directly from the estimates

(a) $(1+\gamma)\left\|v_{1}\right\|+\sqrt{1+\gamma}\left|v_{1}(0)\right| \leqslant C\left\|h_{1}\right\|$,

(b) $\Lambda\left\|v_{2}\right\|+\left\|v_{3}\right\|+\Lambda^{1 / 2}\left|v_{2}(0)\right|+\Lambda^{-1 / 2}\left|v_{3}(0)\right| \leqslant C\left(\left\|h_{2}\right\|+\Lambda^{-1}\left\|h_{3}\right\|\right)$ 
for (3.96), after absorbing a few terms from the right by taking $|\zeta|$ large. It remains just to prove (3.97).

2. The hyperbolic block. Consider the $(N-s) \times(N-s)$ transmission problem

$$
\partial_{z} v_{1}=\widehat{G}^{11} v_{1}+h_{1}, \quad\left[v_{1}\right]=0 \quad \text { on } z=0 .
$$

With notation as in (3.56) let

$$
\Lambda=\operatorname{diag}\left(\mu_{k} I_{s_{k}}+R_{k}\right), \quad v_{1}=\mathcal{V}(z, q, \zeta) w,
$$

and rewrite (3.98)

$$
\begin{aligned}
& \partial_{z} w=\Lambda w+\mathcal{V}^{-1} h_{1}-\mathcal{V}^{-1} \mathcal{V}_{z} w \\
& {[w]=0 \quad \text { on } z=0}
\end{aligned}
$$

On $\pm z \geqslant 0$ we have $R_{k}-R_{k}^{ \pm \infty}=\mathrm{O}\left(e^{-\theta|z|}\right)$ and $\mathcal{V}^{-1} \mathcal{V}_{z}=\mathrm{O}\left(e^{-\theta|z|}\right)$ for $\theta>0$ as in Definition 2.16. Let

$$
w=\left(\mathrm{w}_{1}, \ldots, \mathrm{w}_{k_{0}}\right) \quad \text { and } \quad \mathcal{V}^{-1} h_{1}=\left(\mathrm{h}_{1}, \ldots, \mathrm{h}_{k_{0}}\right) .
$$

Then the equation given by the $k$-th block in (3.100) is, say when $k=1$,

$$
\partial_{z} \mathrm{w}_{1}=\left(\mu_{1}+R_{1}^{ \pm \infty}+\mathrm{O}\left(e^{-\theta|z|}\right)\right) \mathrm{w}_{1}+\mathrm{h}_{1}+\sum_{k>1} \mathrm{O}\left(e^{-\theta|z|}\right) \mathrm{w}_{k} .
$$

Since we want an estimate valid for $0 \leqslant \gamma \leqslant \delta|\zeta|$, we cannot simply absorb the $\mathrm{O}\left(e^{-\theta|z|}\right)$ terms in (3.102) by taking $\gamma$ large. Instead, we take advantage of the exponentially decaying factors by introducing exponential weights $e^{\phi(z)}$ as follows.

Consider the case $-\operatorname{sgn}(\beta)=1$. By Corollary 3.23 we have then

$$
\Re\left(\mu_{k}+R_{k}^{ \pm \infty}\right) \geqslant c(\gamma+1) \quad \text { for all } k .
$$

For a uniformly bounded weight function $\phi(z)$ to be chosen, set

$$
\omega=\left(\omega_{1}, \ldots, \omega_{k_{0}}\right)=e^{\phi} w=e^{\phi}\left(\mathrm{w}_{1}, \ldots, \mathrm{w}_{k_{0}}\right) .
$$

Letting $\omega^{ \pm}=\left.\omega\right|_{ \pm z \geqslant 0}$ we first estimate $\omega^{+}$. From (3.102) we have for $z \geqslant 0$ :

$$
\partial_{z} \omega_{1}^{+}=\left(\mu_{1}+R_{1}^{+\infty}+\mathrm{O}\left(e^{-\theta z}\right)+\phi^{\prime}\right) \omega_{1}^{+}+e^{\phi} \mathrm{h}_{1}+\sum_{k>1} \mathrm{O}\left(e^{-\theta z}\right) \omega_{k}^{+} .
$$

Denote the inner product in $\mathbb{C}^{s_{k}}$ by $\left(\right.$, ). Pairing (3.105) with $\omega_{1}^{+}$and integrating $\int_{0}^{\infty}$ yields

$$
\begin{aligned}
& \left|\omega_{1}^{+}(0)\right|^{2}+2 \Re \int_{0}^{\infty}\left(\left(\mu_{1}+R_{1}^{+\infty}\right) \omega_{1}^{+}, \omega_{1}^{+}\right)+2 \Re \int_{0}^{\infty}\left(\left(\mathrm{O}\left(e^{-\theta z}\right)+\phi^{\prime}\right) \omega_{1}^{+}, \omega_{1}^{+}\right) \\
& =-2 \Re \int_{0}^{\infty}\left(e^{\phi} \mathrm{h}_{1}, \omega_{1}^{+}\right)-2 \Re \int_{0}^{\infty} \sum_{k>1}\left(\omega_{k}^{+}, \mathrm{O}\left(e^{-\theta z}\right) \omega_{1}^{+}\right) .
\end{aligned}
$$

Using (3.103) we find

$$
\begin{aligned}
& \left|\omega_{1}^{+}(0)\right|^{2}+2 c(\gamma+1)\left\|\omega_{1}^{+}\right\|^{2}+2 \Re \int_{0}^{\infty}\left(\left(\mathrm{O}\left(e^{-\theta z}\right)+\phi^{\prime}\right) \omega_{1}^{+}, \omega_{1}^{+}\right) \\
& \leqslant c(\gamma+1)\left\|\omega_{1}^{+}\right\|^{2}+C_{\phi} \frac{\left\|\mathrm{h}_{1}^{+}\right\|^{2}}{\gamma+1}+\alpha\left\|\omega^{+}\right\|^{2}+C_{\alpha}\left\|e^{-\theta z} \omega_{1}^{+}\right\|^{2},
\end{aligned}
$$

where $C_{\alpha}$ depends just on $\alpha$ and $\mathcal{V}$. 
The $\mathrm{O}\left(e^{-\theta z}\right)$ term on the left in (3.106) depends on $R_{1}$ as well as $\mathcal{V}$. Anticipating similar estimates for the other $\omega_{k}^{+}$and noting that $\alpha$ and $C_{\alpha}$ can be fixed independently of $k$ ahead of time, we now choose $\phi$ uniformly bounded on $[0, \infty)$ such that

$$
2 \Re\left(\mathrm{O}\left(e^{-\theta z}\right)+\phi^{\prime}\right) \geqslant C_{\alpha} e^{-2 \theta z} \quad \text { for all } k,
$$

and cancel terms in (3.107) to obtain

$$
\left|\omega_{1}^{+}(0)\right|^{2}+c(\gamma+1)\left\|\omega_{1}^{+}\right\|^{2} \leqslant C_{\phi} \frac{\left\|\mathbf{h}_{1}^{+}\right\|^{2}}{\gamma+1}+\alpha\left\|\omega^{+}\right\|^{2} .
$$

Adding (3.109) to similar estimates for the other components $\omega_{k}^{+}$, we find, provided $\alpha$ was taken small enough,

$$
\sqrt{1+\gamma}\left|\omega^{+}(0)\right|+(\gamma+1)\left\|\omega^{+}\right\| \leqslant C\left\|h_{1}^{+}\right\| .
$$

The boundedness of $\phi$ implies

$$
\sqrt{1+\gamma}\left|w^{+}(0)\right|+(\gamma+1)\left\|w^{+}\right\| \leqslant C\left\|h_{1}^{+}\right\| .
$$

A similar argument on $(-\infty, 0]$ yields

$$
(\gamma+1)\left\|w^{-}\right\| \leqslant C\left(|| h_{1}^{-} \|+\sqrt{1+\gamma}\left|w^{-}(0)\right|\right) .
$$

Combining the estimates (3.111) and (3.112) using $w^{+}(0)=w^{-}(0)$, and recalling $v_{1}=\mathcal{V} w$ gives (3.97)(a) in the case $-\operatorname{sgn}(\beta)=1$. The case $-\operatorname{sgn}(\beta)=-1$ is essentially the same.

Remark 3.30. - The exponentially weighted estimate used for the hyperbolic block is similar to estimates that appear in some papers dealing with the one-dimensional case; see for example $[15,16]$.

3. The parabolic block. To prove (3.97)(b) we set $\tilde{u}=\left(v_{2}, v_{3}\right) \in \mathbb{C}^{2 s}$ and consider the transmission problem

$$
\partial_{z} \tilde{u}=M_{i n}^{22} \tilde{u}+\left(\begin{array}{c}
h_{2} \\
h_{3}
\end{array}\right), \quad[\tilde{u}]=0 \quad \text { on } z=0 .
$$

With $\mathcal{G}=\check{M}_{i n}^{22}$ as in (3.87) and $u=\left(u_{2}, u_{3}\right):=\left(\Lambda_{0}(\zeta) v_{2}, v_{3}\right)$, we have the equivalent problem

$$
\partial_{z} u=\Lambda_{0} \mathcal{G} u+\left(\begin{array}{c}
\Lambda_{0} h_{2} \\
h_{3}
\end{array}\right), \quad[u]=0 \quad \text { on } z=0 .
$$

We can use the spectral projectors $\Pi_{n}, \Pi_{p}$ defined just below (3.88) to construct a smooth conjugator $V(z, q, \check{\zeta}, \lambda)$ for $\lambda \in\left[0, \lambda_{0}\right]$ such that

$$
V^{-1} \mathcal{G} V=\left(\begin{array}{cc}
P_{p} & 0 \\
0 & P_{n}
\end{array}\right)
$$

where the spectrum of $P_{p}$ (respectively $P_{n}$ ) lies in a compact subset of $\Re \mu>0$ (respectively $\Re \mu<0$ ). Modifying $V$ if necessary, we arrange to have

$$
\Re P_{p}>C I_{s}, \quad \Re P_{n}<-C I_{s},
$$

for some $C>0$.

Remark 3.31. - We claim that the conjugator $V(z, q, \check{\zeta}, \lambda)$ can be chosen smoothly for $q$ in a small ball about an arbitrary basepoint $q, \hat{\zeta} \in \check{S}_{+}^{d}=\{\check{\zeta}: \check{\gamma} \geqslant 0\}$, and $\lambda \in\left[0, \lambda_{0}\right]$. Choosing a smooth conjugator is equivalent to choosing a smoothly varying basis for the fibre space of a bundle, so this follows from the fact that contractible base spaces admit only trivial bundles [35, Corollary 11.6].

$4^{e}$ SÉRIE - TOME $39-2006-\mathrm{N}^{\circ} 1$ 
Using \pm to denote functions defined on or restricted to $\pm z \geqslant 0$, we next construct self-adjoint symmetrizers $S^{ \pm}(z, q, \check{\zeta}, \lambda)$ with the properties

$$
\begin{aligned}
& \text { (a) } \Re\left(S^{ \pm} \mathcal{G}^{ \pm}\right) \geqslant I_{2 s} \quad \text { on } \pm z \geqslant 0, \\
& \text { (b) }\left(S^{+} a, a\right)-\left(S^{-} b, b\right) \geqslant|a, b|^{2}-C|\Gamma(a, b)|^{2} \quad \text { on } z=0
\end{aligned}
$$

for some $C>0$, where $\Gamma: \mathbb{C}^{4 s} \rightarrow \mathbb{C}^{2 s}$ is defined by

$$
\Gamma(a, b)=a-b
$$

and $(\cdot, \cdot)$ is the inner product on $\mathbb{C}^{2 s}$. For large enough positive constants $k_{p}^{ \pm}, k_{n}^{ \pm}$, property (3.117)(a) is clearly satisfied by

$$
S^{ \pm}=\left(V_{ \pm}^{-1}\right)^{*}\left(\begin{array}{cc}
k_{p}^{ \pm} I_{s} & 0 \\
0 & -k_{n}^{ \pm} I_{s}
\end{array}\right) V_{ \pm}^{-1} .
$$

To arrange (3.117)(b) observe first that for some positive constants $c, C$, we have on $z=0$ :

$$
\left(S^{+} a, a\right)-\left(S^{-} b, b\right) \geqslant c\left(k_{p}^{+}\left|\Pi_{p} a\right|^{2}+k_{n}^{-}\left|\Pi_{n} b\right|^{2}\right)-C\left(k_{n}^{+}\left|\Pi_{n} a\right|^{2}+k_{p}^{-}\left|\Pi_{p} b\right|^{2}\right) .
$$

For $\mathcal{F}^{\infty}(z, q, \check{\zeta}, \lambda)$ as in (3.89) we have at $z=0$

$$
\left(\Pi_{n} a, \Pi_{p} b\right) \in \mathcal{F}^{\infty}(0, q, \check{\zeta}, \lambda),
$$

so the transversality condition (3.90) implies

$$
\left|\Pi_{n} a, \Pi_{p} b\right| \leqslant C\left|\Gamma\left(\Pi_{n} a, \Pi_{p} b\right)\right| \leqslant C\left(|\Gamma(a, b)|+\left|\Pi_{p} a, \Pi_{n} b\right|\right)
$$

for some $C>0$. Using (3.120) and (3.121) we obtain the property (3.117)(b) by taking the ratios $k_{p}^{+} / k_{n}^{+}$and $k_{n}^{-} / k_{p}^{-}$large enough.

Adding the identities

$$
\begin{aligned}
& \int_{0}^{\infty}\left(\left(2 \Re\left(S^{+} \Lambda_{0} \mathcal{G}^{+}\right)+\partial_{z} S^{+}\right) u^{+}, u^{+}\right) d z+\left(S^{+}(0) u^{+}(0), u^{+}(0)\right) \\
& =-2 \Re \int_{0}^{\infty}\left(S^{+}\left(\begin{array}{c}
\Lambda_{0} h_{2}^{+} \\
h_{3}^{+}
\end{array}\right), u^{+}\right) d z \\
& \int_{-\infty}^{0}\left(\left(2 \Re\left(S^{-} \Lambda_{0} \mathcal{G}^{-}\right)+\partial_{z} S^{-}\right) u^{-}, u^{-}\right) d z-\left(S^{-}(0) u^{-}(0), u^{-}(0)\right) \\
& =-2 \Re \int_{-\infty}^{0}\left(S^{-}\left(\begin{array}{c}
\Lambda_{0} h_{2}^{-} \\
h_{3}^{-}
\end{array}\right), u^{-}\right) d z
\end{aligned}
$$

and using the properties (3.117), we obtain for $|\zeta|$ large the following estimate equivalent to (3.97)(b):

$$
\|u\|+\frac{1}{\Lambda_{0}^{1 / 2}}|u(0)| \leqslant C \frac{1}{\Lambda_{0}}\left\|\Lambda_{0} h_{2}, h_{3}\right\| .
$$

4. The case $\gamma \geqslant C|\zeta|$. Let $C>0$ and consider again the transmission problem (3.91) for $U=\left(u_{1}, u_{2}, u_{3}\right)$ : 


$$
\begin{gathered}
\partial_{z} U-G(z, q, \zeta) U=\left(\begin{array}{c}
g_{1} \\
0 \\
g_{3}
\end{array}\right), \\
{[U]=0 \quad \text { on } z=0 .}
\end{gathered}
$$

For frequencies $\zeta$ such that

$$
\zeta \in \mathcal{Z}:=\{\zeta: \gamma \geqslant C|\zeta|,|\zeta| \geqslant R\}
$$

we will prove a stronger estimate than (3.92), namely

$$
\begin{aligned}
& |\zeta|\left\|u_{1}\right\|+\Lambda\left\|u_{2}\right\|+\left\|u_{3}\right\|+\sqrt{|\zeta|}\left|u_{1}(0)\right|+\Lambda^{1 / 2}\left|u_{2}(0)\right|+\Lambda^{-1 / 2}\left|u_{3}(0)\right| \\
& \quad \leqslant C\left(\left\|g_{1}\right\|+\Lambda^{-1}\left\|g_{3}\right\|\right) .
\end{aligned}
$$

The argument now does not require any subtle conjugations or exponential weights like $\phi(z)$ in (3.104). With the larger weight on $\left\|u_{1}\right\|$ we can easily absorb larger errors.

Let $G_{p}^{11}$ be the principal part, homogeneous of degree one in $\zeta$, of $G^{11}$. First rewrite (3.124) as

$$
\begin{aligned}
& \partial_{z} U-\left(\begin{array}{ccc}
G_{p}^{11} & 0 & 0 \\
0 & 0 & I \\
0 & G^{32} & G^{33}
\end{array}\right) U \\
& =\left(\begin{array}{c}
g_{1}+\mathrm{O}(1) u_{1}+\mathrm{O}(|\eta|) u_{2}+\mathrm{O}(1) u_{3} \\
0 \\
g_{3}+\mathrm{O}(|\zeta|) u_{1}
\end{array}\right):=\left(\begin{array}{c}
h_{1} \\
0 \\
h_{3}
\end{array}\right) .
\end{aligned}
$$

The estimate (3.125) will follow directly by adding the estimates

$$
\begin{aligned}
|\zeta|\left\|u_{1}\right\|+\sqrt{|\zeta|}\left|u_{1}(0)\right| & \leqslant C\left\|h_{1}\right\|, \\
\Lambda\left\|u_{2}\right\|+\left\|u_{3}\right\|+\Lambda^{1 / 2}\left|u_{2}(0)\right|+\Lambda^{-1 / 2}\left|u_{3}(0)\right| & \leqslant C \Lambda^{-1}\left\|h_{3}\right\|
\end{aligned}
$$

after absorbing a few error terms from the right by taking $|\zeta| \geqslant R$ large enough. In particular to absorb the $\Lambda^{-1} \mathrm{O}(|\zeta|)\left\|u_{1}\right\|$ term we use $\Lambda(\zeta) \geqslant \sqrt{|\zeta|}$.

It remains to prove (3.127). The $\left(u_{2}, u_{3}\right)$ estimate is done exactly as before.

Before estimating $u_{1}$, we note that for $\gamma \geqslant C|\zeta|$ we do not know that the eigenvalues $\mu_{k}$ of $G_{p}^{11}$ are semisimple with constant multiplicities. Hyperbolicity of $\bar{A}^{11}$ implies $\Re \mu_{k} \neq 0$ whenever $\gamma>0$, so by homogeneity we have

$$
\left|\Re \mu_{k}(z, q, \zeta)\right| \geqslant c|\zeta| \text { for } \gamma \geqslant C|\zeta| .
$$

This is the simplest "elliptic" case for the construction of Kreiss symmetrizers.

From Proposition 3.20 we deduce that for $\zeta \in \mathcal{Z}, \Re \mu_{k}$ has the same fixed sign for all $k$, namely $-\operatorname{sgn}(\beta)$, where $\beta$ is any eigenvalue of $\bar{A}_{d}^{11}(z, q)$. We take $-\operatorname{sgn}(\beta)=1$, the other case being similar.

The argument is closely parallel to the earlier treatment of the parabolic block, but simpler. Let $\zeta=|\zeta| \hat{\zeta}$ and write $G_{p}^{11}=|\zeta| \mathcal{G}^{11}$. There is a smooth conjugator $V(z, q, \hat{\zeta})$ such that

$$
V^{-1} \mathcal{G}^{11} V=\mathbb{G}^{11}, \quad \text { where } \Re \mathbb{G}^{11} \geqslant C I_{N-s}
$$

for some $C>0$. The positive and negative invariant spaces of $\mathcal{G}^{11}$ are respectively,

$$
\mathbb{F}_{p}(z, q, \hat{\zeta})=\mathbb{C}^{N-s} \quad \text { and } \quad \mathbb{F}_{n}(z, q, \hat{\zeta})=0
$$

and, defining

$4^{\text {e }}$ SÉRIE - TOME $39-2006-\mathrm{N}^{\circ} 1$ 


$$
\Gamma: \mathbb{C}^{2(N-s)} \rightarrow \mathbb{C}^{N-s}, \quad \Gamma(a, b)=a-b,
$$

we have the exact analogue of Corollary 3.28.

We construct symmetrizers

$$
S^{ \pm}(z, q, \hat{\zeta})=\left(V_{ \pm}^{-1}\right)^{*}\left(k_{p}^{ \pm} I_{N-s}\right) V_{ \pm}^{-1},
$$

where $k_{p}^{+}>0$ is large enough and $k_{p}^{-}=1$, satisfying

$$
\begin{aligned}
& \Re\left(S^{ \pm} \mathcal{G}_{ \pm}^{11}\right) \geqslant I_{N-s} \quad \text { on } \pm z \geqslant 0, \\
& \left(S^{+} a, a\right)-\left(S^{-} b, b\right) \geqslant|a, b|^{2}-C|\Gamma(a, b)|^{2} \quad \text { on } z=0 .
\end{aligned}
$$

Repetition of the proof for the parabolic block gives the estimate

$$
|\zeta||| u_{1}\left\|+\sqrt{|\zeta|}\left|u_{1}(0)\right| \leqslant C\right\| h_{1} \|
$$

in place of (3.123).

Remark 3.32. - As we explain later in the proof of Proposition 6.6, the fully linearized operator that we actually use in the nonlinear iteration scheme has a principal part in the high frequency regime that differs slightly (after freezing coefficients, Fourier transforming, and rescaling) from the operator appearing in (3.3):

$$
\mathcal{L}\left(z, q, \zeta, \partial_{z}\right) u-\psi \mathcal{K}(z, q, \zeta)=f .
$$

The correct high frequency operator is defined by replacing $\mathcal{L}$ in (3.135) by $\widetilde{\mathcal{L}}$, where $\widetilde{\mathcal{L}}$ is defined just like $\mathcal{L}$ except that the $(2,1)$ entry of the matrix coefficient $\mathbb{A}_{d}(z, q)(3.15)$ is replaced by

$$
\widetilde{\mathbb{A}}_{d}^{21}=\mathbb{A}_{d}^{21}(z, q)+r
$$

where $r$ is a (frozen) $s \times(N-s)$ matrix of small norm.

The identity in Lemma 2.28 now becomes:

$$
\widetilde{\mathcal{L}} \partial_{z} W=\mathcal{K}-\partial_{z} \mathcal{P}+\left(\begin{array}{c}
0 \\
r \partial_{z}^{2} W_{1}
\end{array}\right)
$$

With this change the reduction carried out in Section 3.2.1 goes through just as before, except that the partially linearized problem is now

$$
\begin{gathered}
\widetilde{\mathcal{L}} u=f \\
{[u]=0, \quad\left[\partial_{z} u_{2}\right]=0 \quad \text { on } z=0}
\end{gathered}
$$

instead of (3.8), and (3.11)(a) should be replaced by

$$
\widetilde{\mathcal{L}} u^{\#}=f+\psi \partial_{z} \mathcal{P}-\psi\left(\begin{array}{c}
0 \\
r \partial_{z}^{2} W_{1}
\end{array}\right)
$$

where the profile $W=\left(W_{1}, W_{2}\right)$.

Moreover, for arbitrary sufficiently small $r$ all the constructions and estimates of Sections 3.2.2 to 3.2.7 go through exactly as before for the new problem (3.138). This is because the change in $\mathbb{A}_{d}^{21}$ has no effect on the validity of hypotheses (H2), (H3), and (H8). Also, Corollary 4.1 implies that with such a change, hypothesis (H5) still holds for $|\xi|$ large. The hyperbolicity hypothesis (H4) may no longer hold, but (H4) was not used in Section 3.2.

It is convenient then to redefine the parameter $q=\left(p, u^{\prime}, h^{\prime}\right)(2.24)$ to include $r$. So henceforth

$$
q=\left(p, u^{\prime}, h^{\prime}, r\right)
$$


and we drop the tildes on $\mathcal{L}$ and $\mathbb{A}_{d}^{21}$. When working in the low and medium frequency regimes, we always take $r=0$. This reflects the fact, demonstrated in Section 4.4, that the perturbation produced by $r_{\varepsilon}$ in the low and medium frequency variable coefficient estimates is a negligible one.

\subsection{Low and medium frequency estimates}

In this section we discuss the estimates of Propositions 3.2 and 3.3 for the fully linearized transmission problem (3.3):

$$
\begin{gathered}
\mathcal{L}\left(z, q, \zeta, \partial_{z}\right) u-\psi \mathcal{K}(z, q, \zeta)=f, \\
{[u]=0, \quad\left[\partial_{z} u_{2}\right]=0, \quad a(\zeta) \psi+\ell(q) \cdot u_{2}(0)=0 \quad \text { on } z=0 .}
\end{gathered}
$$

The low and medium frequency analysis for the systems of Navier-Stokes type that we study in this paper has much in common with the analysis of [12] for the fully parabolic case; so we will give proofs when different arguments are needed, but otherwise shall refer to previous work. We first describe the reduction to the partially linearized problem for this range of frequencies. All the results depend on the conjugations to limiting and block forms explained later.

\subsubsection{Reduction to the partially linearized case}

In the medium frequency regime the same choice of good unknown that worked for high frequencies,

$$
u^{\#}=u-\psi \partial_{z} W,
$$

allows us to prove estimate (3.7) for solutions $(u, \psi)$ of the fully linearized problem (3.3) by showing that solutions of the partially linearized problem,

$$
\begin{gathered}
\mathcal{L}\left(z, q, \zeta, \partial_{z}\right) u=f, \\
{[u]=0, \quad\left[\partial_{z} u_{2}\right]=0 \quad \text { on } z=0,}
\end{gathered}
$$

satisfy

$$
\|u\|+\left\|\partial_{z} u_{2}\right\|+|u(0)|+\left|\partial_{z} u_{2}(0)\right| \leqslant C\left\|f_{1}, f_{2}\right\| .
$$

The proof of (3.144) does not differ essentially from that of [30, Lemma 2.12]. This works because the Evans assumption (H10) implies that the Lopatinski determinant for the transmission problem (3.143) is nonvanishing for medium frequencies. In fact, for $\zeta \neq 0$ the latter determinant, which is given by $(2.54)$

$$
\operatorname{det}\left(\mathbb{E}^{-}(q, \zeta) \times \mathbb{E}^{+}(q, \zeta), \operatorname{ker} \Gamma\right),
$$

is equal to the Evans determinant $D(q, \zeta)$. Here $\Gamma: \mathbb{C}^{N+s} \times \mathbb{C}^{N+s}$ is given by

$$
\Gamma\left(U^{-}, U^{+}\right)=U^{+}-U^{-} .
$$

The argument that accomplishes the reduction to (3.144) is just as in the high frequency case; recall (3.11).

Note that the problem (3.143) is singular at $\zeta=0$. Indeed, when $q=(p, 0,0)$ for $p \in \mathcal{C}$, the derivative of the shock profile, $\partial_{z} W_{0}(z, p)$, is a nontrivial solution for $f=0$ that decays exponentially fast as $z \rightarrow \pm \infty$. Thus, a more subtle choice of good unknown is needed in order to reduce to a nonsingular partially linearized problem for low frequencies. We define the new unknown using the next lemma.

Recall that in the low and medium frequency regions we define $q=\left(p, u^{\prime}, h^{\prime}\right)$, where $p \in \mathcal{C}$ and $\left(u^{\prime}, h^{\prime}\right) \in \mathbb{R}^{N} \times \mathbb{R}^{d}$ as in (2.24).

$4^{e}$ SÉRIE - TOME $39-2006-\mathrm{N}^{\circ} 1$ 
LEMmA 3.33. - (See [12, Lemma 3.14].) Let $\underline{p} \in \mathcal{C}$ and $\operatorname{set} \underline{q}=(\underline{p}, 0,0)$ and take $\underline{\zeta}=0$. There are a neighborhood $\Omega$ of $(\underline{q}, 0)$ and $C^{\infty}$ functions $R^{ \pm}(z, q, \zeta)=\left(\bar{R}_{1}^{ \pm}, R_{2}^{ \pm}\right)$on $\{ \pm \bar{z} \geqslant 0\} \times \Omega$, respectively, such that

$$
\begin{gathered}
\mathcal{L}\left(z, q, \zeta, \partial_{z}\right) R^{ \pm}=c K^{ \pm}(z, q, \zeta) \quad \text { on } \pm z \geqslant 0, \\
\ell(q) \cdot R_{2}^{ \pm}(0, q, \zeta)=-a(\zeta), \quad R^{ \pm}(z, q, 0)=0 .
\end{gathered}
$$

Moreover, $R^{ \pm}$and all their derivatives are exponentially decaying as $z \rightarrow \pm \infty$.

For $(u, \psi)$ as in (3.141) define the good unknown

$$
\mu^{ \pm}=u^{ \pm}-\psi R^{ \pm}
$$

Then $(u, \psi)$ satisfies (3.141) if and only if $\mu$ satisfies the transmission problem:

$$
\begin{gathered}
\mathcal{L} \mu=f, \\
{[\mu]=-\psi[R], \quad\left[\partial_{z} \mu_{2}\right]=-\psi\left[\partial_{z} R_{2}\right], \quad \ell(q) \cdot \mu_{2}^{+}=0 \quad \text { on } z=0 .}
\end{gathered}
$$

Observe that by (3.147), $\ell(q) \cdot \mu_{2}^{+}(0)=0 \Longleftrightarrow \ell(q) \cdot \mu_{2}^{-}(0)=0$.

The next step is to eliminate the front and obtain nonsingular boundary conditions for $\mu$ alone. Since $R^{ \pm}(z, q, 0)=0$ we can use polar coordinates to write

$$
R^{ \pm}(z, q, \zeta)=\rho \widehat{R}^{ \pm}(z, q, \hat{\zeta}, \rho) .
$$

Setting $\phi:=\rho \psi$ we can rewrite the transmission conditions in (3.149) as

$$
[\mu]=-\phi[\widehat{R}], \quad\left[\partial_{z} \mu_{2}\right]=-\phi\left[\partial_{z} \widehat{R}_{2}\right], \quad \ell(q) \cdot \mu_{2}^{+}=0 \quad \text { on } z=0 .
$$

Define the $\mathbb{C}^{N+s}$-valued function

$$
\mathcal{R}(z, q, \hat{\zeta}, \rho)=\left(\widehat{R}, \partial_{z} \widehat{R}_{2}\right) .
$$

The following proposition is a consequence of uniform stability of the shock profile $W_{0}(z, \underline{p})$.

PROPOSITION 3.34. - (See [12, Proposition 3.15].) There is a neighborhood $\omega$ of $\underline{q}=(\underline{p}, 0,0)$ and $\rho_{0}>0$ such that $[\mathcal{R}]$ does not vanish on $\omega \times \bar{S}_{+}^{d} \times\left[0, \rho_{0}\right]$. Here $\bar{S}_{+}^{d}=\{\zeta:|\zeta|=1, \gamma \geqslant 0\}$.

Thus, we may define a smooth orthogonal projector $\pi(q, \hat{\zeta}, \rho): \mathbb{C}^{N+s} \rightarrow \mathbb{C}^{N+s}$ onto $[\mathcal{R}(0, q, \hat{\zeta}, \rho)]^{\perp}$. Applying $\pi$ to the jump condition in (3.151) we obtain a transmission problem for $\mu$ with new transmission conditions:

$$
\begin{gathered}
\mathcal{L}\left(z, q, \zeta, \partial_{z}\right) \mu=f \\
\pi(q, \hat{\zeta}, \rho)\left(\begin{array}{c}
{[\mu]} \\
{\left[\partial_{z} \mu_{2}\right]}
\end{array}\right)=0, \quad \ell(q) \cdot \mu_{2}^{+}=0 \quad \text { on } z=0 .
\end{gathered}
$$

We claim that the Lopatinski determinant for this problem, $D_{\pi}(q, \hat{\zeta}, \rho)$, is bounded away from 0 uniformly for $\rho$ small. To define $D_{\pi}$ let $U=\left(U_{1}, U_{2}, U_{3}\right) \in \mathbb{C}^{N+s}$ with $U_{1} \in \mathbb{C}^{N-s}, U_{3} \in \mathbb{C}^{s}$ and define $\Gamma_{\pi}(q, \hat{\zeta}, \rho): \mathbb{C}^{N+s} \times \mathbb{C}^{N+s} \rightarrow \mathbb{C}^{N+s} \times \mathbb{C}$ by

$$
\Gamma_{\pi}\left(U^{-}, U^{+}\right)=\left(\pi(q, \hat{\zeta}, \rho)\left(U^{+}-U^{-}\right), \ell(q) \cdot U_{2}^{+}\right) .
$$

Then for $0<\rho \leqslant \rho_{0}$ the Lopatinski determinant for the transmission problem (3.153) is

$$
D_{\pi}(q, \hat{\zeta}, \rho):=\operatorname{det}\left(E^{-}(q, \hat{\zeta}, \rho) \times E^{+}(q, \hat{\zeta}, \rho), \operatorname{ker} \Gamma_{\pi}(q, \hat{\zeta}, \rho)\right)
$$

Lemma 2.19 implies that the dimensions of $E^{ \pm}$sum to $N+s$ and $\operatorname{dim} \operatorname{ker} \Gamma_{\pi}=N+s$, so the determinant makes sense. 
In fact the argument of [31, Theorem 3.3] shows that $E^{ \pm}(q, \hat{\zeta}, \rho)$, which are $C^{\infty}$ vector bundles on $\omega \times \bar{S}_{+}^{d} \times(0, \infty)$ by Lemma 2.19 , extend continuously to $\omega \times \bar{S}_{+}^{d} \times[0, \infty)$. Thus, $D_{\pi}$ extends continuously to $\omega \times \bar{S}_{+}^{d} \times\left[0, \rho_{0}\right]$. The uniform stability of the shock profile $W_{0}(z, \underline{p})$ implies, by the argument of [12, Propositions 3.15 and 3.16]:

PROPOSITION 3.35. - There is a neighborhood $\omega$ of $\underline{q}=(\underline{p}, 0,0)$ and positive constants $\rho_{0}$, c such that

$$
\left|D_{\pi}(q, \hat{\zeta}, \rho)\right| \geqslant c \quad \text { on } \omega \times \bar{S}_{+}^{d} \times\left[0, \rho_{0}\right]
$$

This should be contrasted with the first-order vanishing of the Evans function $D(q, \zeta)$ at $\zeta=0$. Thus, the choice of the unknown $\mu$ and the extra boundary condition in (3.141) has allowed us to remove the translational degeneracy. Once the conjugations described in the next subsection are performed, one can proceed to construct symmetrizers for the transmission problem (3.153) and derive the estimate

$$
\lambda^{2}\|\mu\|+\lambda\left\|\partial_{z} \mu_{2}\right\|+\lambda|\mu(0)|+\lambda 2\left|\partial_{z} \mu_{2}(0)\right| \leqslant C\left\|f_{1}, f_{2}\right\|,
$$

where $\lambda(\zeta)=\left(\gamma+|\zeta|^{2}\right)^{1 / 2}$.

Remark 3.36. - To prove (3.156) it suffices, by continuity, to prove

$$
D_{\pi}(\underline{q}, \hat{\zeta}, 0) \neq 0 \quad \text { for } \hat{\zeta} \in S_{+}^{d} .
$$

The first-order vanishing of the Evans function $D(\underline{q}, \hat{\zeta}, \rho)$ at $\rho=0$ as assumed in (H9) implies

$$
\begin{aligned}
& \left(E^{-}(\underline{q}, \hat{\zeta}, 0) \times E^{+}(\underline{q}, \hat{\zeta}, 0)\right) \cap \operatorname{ker} \Gamma \\
& \quad=\operatorname{span}\left\{\left(\partial_{z} W(0, \underline{q}), \partial_{z}^{2} W_{2}(0, \underline{q})\right),\left(\partial_{z} W(0, \underline{q}), \partial_{z}^{2} W_{2}(0, \underline{q})\right)\right\} .
\end{aligned}
$$

We have $\ell(\underline{q}) \cdot \partial_{z} W_{2}(0, \underline{q}) \neq 0$, so no nontrivial element $\left(U^{-}, U^{+}\right)$of $E^{-}(\underline{q}, \hat{\zeta}, 0) \times E^{+}(\underline{q}, \hat{\zeta}, 0)$ satisfying $\ell(\underline{q}) \cdot U_{2}^{+}=0$ can belong to $\operatorname{ker} \Gamma$. To prove (3.158) one must show that $\left(U^{-}, U^{+}\right)$ does not belong to the larger space $\operatorname{ker}(\pi(\underline{q}, \hat{\zeta}, 0) \Gamma)$; this follows from uniform stability of the inviscid shock.

The transmission condition (3.151) and nonvanishing of $[\mathcal{R}]$ imply

$$
\rho|\psi| \leqslant C\left(|\mu(0)|+\left|\partial_{z} \mu_{2}(0)\right|\right) .
$$

Using (3.160) and the exponential decay of $R^{ \pm}$as $z \rightarrow \pm \infty$ respectively, we immediately derive the low frequency estimate of Proposition 3.2 for the fully linearized transmission problem (3.141) from the estimate (3.157).

\subsubsection{Conjugation to a limiting constant coefficient problem}

We have seen that the partially linearized transmission problem (3.143) can be written in first order form as

$$
\partial_{z} U-G(z, q, \zeta) U=\mathcal{J} f, \quad \Gamma U=[U]=0
$$

for $G$ as in (3.35) and $\mathcal{J}$ as in (3.33). Recalling that for $q=\left(p, u^{\prime}, h^{\prime}\right)$ and $W(z, q)=$ $W_{0}(z, p)+u^{\prime}$ we have

$$
\lim _{z \rightarrow \pm \infty} W(z, q)=u^{ \pm}+u^{\prime}
$$

so we can define the limiting matrices

$4^{\mathrm{e}}$ SÉRIE - TOME $39-2006-\mathrm{N}^{\circ} 1$ 


$$
G_{ \pm}(q, \zeta):=\lim _{z \rightarrow \pm \infty} G(z, q, \zeta) .
$$

A key step in the construction of symmetrizers for bounded frequencies is the local conjugation of (3.163) to a problem defined by $G_{ \pm}$provided by the following lemma.

Lemma 3.37. - (See [30, Lemma 2.6].) Let $\mathcal{Q}=\mathcal{C} \times \mathcal{O}$ as in (2.52). For each $\underline{q} \in \mathcal{Q}$ and $\underline{\zeta} \in \overline{\mathbb{R}}_{+}^{d+1}$ there is a neighborhood $\Omega$ of $(\underline{q}, \underline{\zeta})$ in $\mathcal{Q} \times \overline{\mathbb{R}}_{+}^{d+1}$ and there are matrices $\mathcal{W}_{ \pm}$defined and $C^{\infty}$ on $\{ \pm z \geqslant 0\} \times \Omega$ satisfying:

(a) $\mathcal{W}_{ \pm}$and $\left(\mathcal{W}_{ \pm}\right)^{-1}$ are uniformly bounded and there is a $\delta_{0}>0$ such that for $(q, \zeta) \in \Omega$ and all $\alpha$,

$$
\left|\partial_{z, q, \zeta}^{\alpha}\left(\mathcal{W}_{ \pm}(z, q, \zeta)-\mathrm{Id}\right)\right| \lesssim e^{-\delta_{0}|z|} \quad \text { on } \pm z \geqslant 0 .
$$

(b) $\mathrm{On} \pm z \geqslant 0$ we have, respectively

$$
\partial_{z} \mathcal{W}_{ \pm}(z, q, \zeta)=G(z, q, \zeta) \mathcal{W}_{ \pm}(z, q, \zeta)-\mathcal{W}_{ \pm}(z, q, \zeta) G_{ \pm}(q, \zeta)
$$

An immediate corollary is that $U$ satisfies (3.161) if and only if $V_{ \pm}:=\left(\mathcal{W}_{ \pm}\right)^{-1} U$ satisfies

$$
\begin{array}{cl}
\partial_{z} V_{ \pm}=G_{ \pm} V_{ \pm}+\left(\mathcal{W}_{ \pm}\right)^{-1} \mathcal{J} f & \text { on } \pm z \geqslant 0, \\
\Gamma_{w} V:=\mathcal{W}_{+} V_{+}-\mathcal{W}_{-} V_{-}=0 & \text { on } z=0 .
\end{array}
$$

\subsubsection{Spectral properties of $G_{ \pm}(q, \zeta)$}

The entries of $G_{ \pm}(q, \zeta)$ are given by the same formulas as the $G^{i j}(z, q, \zeta)$ (3.36), except that now $E=0, \mathbb{A}_{j}=\widetilde{A}_{j}$, and matrix coefficients are evaluated at $(z, q)=( \pm \infty, q)$, respectively.

Notation 3.38. - We will sometimes write $\left(\bar{A}_{j}\right)_{ \pm}(q)=\bar{A}_{j}( \pm \infty, q)$ and use similar notation for the $\widetilde{A}_{j}, \bar{B}_{j, k}$, etc.

In place of $\overline{\mathcal{L}}(z, q, \tau-i \gamma, \xi)=A_{0}^{-1} \mathcal{L}$ as in (3.16) we have now in the limit as $z \rightarrow \pm \infty$,

$$
\overline{\mathcal{L}}_{ \pm}(q, \tau-i \gamma, \xi)=(i \tau+\gamma) I+i \bar{A}_{ \pm}(q, \xi)+\bar{B}_{ \pm}(q, \xi),
$$

where $\xi=\left(\eta, \xi_{d}\right)$.

Lemma 3.39 (The case $\zeta \neq 0$ ). - Assume (H2), (H5), (H8). Let $H$ be the number of positive eigenvalues of $\bar{A}_{d}^{11}(z, q)$. For $q \in \mathcal{Q}$ and $\zeta \in \overline{\mathbb{R}}_{+}^{d+1} \backslash 0$ the matrices $G_{ \pm}(q, \zeta)$ have no eigenvalues on the imaginary axis. $G_{+}(q, \zeta)$ has $s+H$ eigenvalues counted with multiplicity in $\Re \mu<0$, and $G_{-}(q, \zeta)$ has $N-H$ eigenvalues counted with multiplicity in $\Re \mu>0$.

Proof. - 1. To see that $G_{+}(q, \zeta)$ has no eigenvalue on the imaginary axis, note that $\mu$ is an eigenvalue of $G_{+}(q, \zeta)$ if and only if $-(i \tau+\gamma)$ is an eigenvalue of $i \bar{A}_{+}(q, \xi)+\bar{B}_{+}(q, \xi)$ with $\xi=(\eta,-i \mu)$. Now $\gamma \geqslant 0$, so if $\mu$ is purely imaginary, strict dissipativity (H5) implies $\gamma=0$ and $\xi=0$, and thus $\zeta$ must be 0 .

2. From above we conclude that the number of eigenvalues of $G_{+}$in $\Re \mu<0$ is independent of $\zeta \in \overline{\mathbb{R}}_{+}^{d+1} \backslash 0$. To count them it is easiest to consider $\zeta=(0, \gamma, 0)$ with $\gamma$ large [36]. Again writing $U=\left(U_{1}, U_{2}, U_{3}\right)$, the eigenvalue problem $\left(\mu I-G_{+}(q, \zeta)\right) U=0$ can for such $\zeta$ be rewritten using $\overline{\mathcal{L}}_{+}$as

$$
\left(\begin{array}{cc}
\mu \bar{A}_{d}^{11}+\gamma I & \mu \bar{A}_{d}^{12} \\
\mu \bar{A}_{d}^{21}+\gamma I & \mu \bar{A}_{d}^{22}-\mu^{2} \bar{B}_{d, d}^{22}+\gamma I
\end{array}\right)_{+}\left(\begin{array}{l}
U_{1} \\
U_{2}
\end{array}\right)=\left(\begin{array}{l}
0 \\
0
\end{array}\right) .
$$

We look first for $\mu=\tilde{\mu}(\gamma) \gamma$ where $\tilde{\mu}(\gamma) \sim 1$. A perturbation argument yields $N-s$ such solutions close to solutions $\mu_{*}$ of the problem 


$$
\left(\begin{array}{cc}
\mu_{*} \bar{A}_{d}^{11}+I & \mu_{*} \bar{A}_{d}^{12} \\
0 & -\mu_{*}^{2} \bar{B}_{d, d}^{22}
\end{array}\right)_{+}\left(\begin{array}{l}
U_{1} \\
U_{2}
\end{array}\right)=\left(\begin{array}{l}
0 \\
0
\end{array}\right),
$$

that is, close to $\mu_{*}$ such that $-\mu_{*}^{-1} \in \operatorname{spec}\left(\bar{A}_{d}^{11}\right)$. This gives $H$ eigenvalues of $G_{+}$in $\Re \mu<0$.

Next, looking for solutions $\mu=\tilde{\mu}(\gamma) \gamma^{1 / 2}$ with $\tilde{\mu}(\gamma) \sim 1$, we similarly obtain $2 s$ solutions close to solutions $\mu_{*}$ of the problem

$$
\left(\begin{array}{cc}
I & 0 \\
I & -\mu_{*}^{2} \bar{B}_{d, d}^{22}+I
\end{array}\right)\left(\begin{array}{l}
U_{1} \\
U_{2}
\end{array}\right)=\left(\begin{array}{l}
0 \\
0
\end{array}\right)
$$

that is, close to $\mu_{*}$ such that $\mu_{*}^{-2} \in \operatorname{spec} \bar{B}_{d, d}^{22}$. This gives $s$ additional eigenvalues of $G_{+}$in $\Re \mu<0$, for a total of $s+H$.

The same argument for $G_{-}$yields $(N-s-H)+s=N-H$ eigenvalues in $\Re \mu>0$.

An application of the previous two lemmas is:

Proof of Lemma 2.19. - For $\zeta \in \overline{\mathbb{R}}^{d+1} \backslash 0$ let $\mathbb{F}^{ \pm}(q, \zeta)$ denote, respectively, the generalized eigenspace of $G^{ \pm}(q, \zeta)$ associated to eigenvalues in $\{ \pm \Re \mu<0\}$. By Lemma 3.39 we have

$$
\operatorname{dim} \mathbb{F}^{+}(q, \zeta)=s+H, \quad \operatorname{dim} \mathbb{F}^{-}(q, \zeta)=N-H .
$$

Using the properties of $\mathcal{W}_{ \pm}(0, q, \zeta)$ in Lemma 3.37 and in particular (3.165), we see

$$
\mathbb{E}^{ \pm}(q, \zeta)=\mathcal{W}_{ \pm}(0, q, \zeta) \mathbb{F}^{ \pm}(q, \zeta),
$$

so Lemma 2.19 follows with $K=s+H$.

Lemma 3.40 (The case $\zeta=0)$. - Assume (H2), (H5), and (H8). For $q \in \mathcal{Q}$ each of $G_{ \pm}(q, 0)$ has 0 as a semisimple eigenvalue of multiplicity $N$ and s eigenvalues, counted with multiplicities, in $\Re \mu \neq 0$.

Proof. - Inspection of the formulas for the $G^{i j}$ (3.36) shows that

$$
G_{+}(q, 0)=\left(\begin{array}{ccc}
0 & 0 & * \\
0 & 0 & I \\
0 & 0 & P_{0}
\end{array}\right)_{+},
$$

where

$$
P_{0}^{+}(q)=\left(\widetilde{B}_{d, d}^{22}\right)^{-1}\left(\widetilde{A}_{d}^{22}-\widetilde{A}_{d}^{21}\left(\widetilde{A}_{d}^{11}\right)^{-1} \widetilde{A}_{d}^{12}\right)_{+} .
$$

$P_{0}^{+}$is nonsingular because $\widetilde{B}_{d, d}^{22}, \widetilde{A}_{d}$, and $\widetilde{A}_{d}^{11}$ are, so we observe from (3.172) that 0 is a semisimple eigenvalue of $G_{+}(q, 0)$ of multiplicity $N$.

On the other hand, if $\mu$ is a purely imaginary eigenvalue of $G_{+}(q, 0)$, then 0 is an eigenvalue of $i \bar{A}_{+}(q, \xi)+\bar{B}_{+}(q, \xi)$ with $\xi=(0,-i \mu)$. By strict dissipativity (H5) this requires that $\xi=0$, and thus $\mu=0$. So the nonvanishing eigenvalues of $G_{+}(q, 0)$, which are the eigenvalues of $P_{0}^{+}(q)$, do not lie on the imaginary axis.

The argument for $G_{-}(q, 0)$ is the same.

\subsubsection{Conjugation to block form}

Another essential step in the construction of symmetrizers is the conjugation of $G_{ \pm}$to appropriate block forms. We will derive such conjugations in the medium and low frequency regions as consequences of Lemmas 3.39 and 3.40. This is quite easy for medium frequencies.

$4^{e}$ SÉRIE - TOME $39-2006-\mathrm{N}^{\circ} 1$ 
Proposition 3.41 (Medium frequency). - For $\underline{q} \in \mathcal{Q}$ and $\underline{\zeta} \in \overline{\mathbb{R}}_{+}^{d+1} \backslash 0$ there is a neighborhood $\Omega$ of $(\underline{q}, \underline{\zeta})$ and a $C^{\infty}$ invertible matrix $\overline{\mathcal{V}}_{+}(q, \zeta)$ on $\Omega$ such that

$$
\mathcal{V}_{+}^{-1} G_{+} \mathcal{V}_{+}=\left(\begin{array}{cc}
G_{p}^{+} & 0 \\
0 & G_{n}^{+}
\end{array}\right) \text {on } \Omega
$$

where

$$
\Re G_{p}^{+}>0, \quad \Re G_{n}^{+}<0 .
$$

The same result holds with '+' replaced by '-'.

Proof. - The spectral separation demonstrated in Lemma 3.39 implies the existence of a smooth $\mathcal{V}$ such that (3.174) holds with matrices $G_{p}^{+}, G_{n}^{+}$whose eigenvalues $\mu$ all satisfy, respectively, $\Re \mu>0, \Re \mu<0$. Modifying $\mathcal{V}$ if necessary (e.g., as in [4, Chapter 6, Lemma 5.5]) we obtain (3.175).

Remark 3.42 (Medium frequency estimate). - Given (3.174), the construction of symmetrizers for the constant coefficient problem (3.165) in the medium frequency region proceeds just as in the treatment of the parabolic block in Section 3.2. Note that the properties of the conjugators $\mathcal{W}_{ \pm}$imply that the Lopatinski determinant for (3.165) is nonvanishing since the determinant (3.145) is. The properties of $\mathcal{W}_{ \pm}$allow the estimate (3.144) for the problem (3.143) to be deduced immediately from the same estimate for the conjugated problem (3.165).

In preparation for the next proposition we rewrite $\overline{\mathcal{L}}(q, \tau-i \gamma, \xi)$ in (3.166) as

$$
\overline{\mathcal{L}}(q, \tau-i \gamma, \xi)=\left(\bar{B}_{d, d}(q) \xi_{d}^{2}+\mathcal{A}(q, \zeta) i \xi_{d}+\mathcal{M}(q, \zeta)\right)_{ \pm},
$$

where $\xi=\left(\eta, \xi_{d}\right)$ and

$$
\begin{aligned}
& \mathcal{A}_{ \pm}(q, \zeta)=\left(\bar{A}_{d}-\sum_{j=1}^{d-1} i \eta_{j}\left(\bar{B}_{j, d}+\bar{B}_{d, j}\right)\right)_{ \pm} \\
& \mathcal{M}_{ \pm}(q, \zeta)=\left((i \tau+\gamma) I+\sum_{j=1}^{d-1} i \eta_{j} \bar{A}_{j}+\sum_{j, k=1}^{d-1} \eta_{j} \eta_{k} \bar{B}_{j, k}\right)_{ \pm} .
\end{aligned}
$$

The system $\overline{\mathcal{L}}\left(q, \zeta, D_{z}\right) u=f$ reads, with $U=\left(u, \partial_{z} u_{2}\right)$ :

$$
\begin{aligned}
& G_{d} \partial_{z} U-M U=\left(\begin{array}{l}
f \\
0
\end{array}\right):=F, \\
& G_{d}(q, \zeta)=\left(\begin{array}{cc}
-\mathcal{A} & \bar{B}_{d}(q) \\
J & 0
\end{array}\right), \quad M=\left(\begin{array}{cc}
\mathcal{M} & 0_{N \times s} \\
0_{s \times N} & I_{s \times s}
\end{array}\right),
\end{aligned}
$$

where

$$
\bar{B}_{d}(q)=\left(\begin{array}{c}
0_{(N-s) \times s} \\
\bar{B}_{d, d}^{22}(q)
\end{array}\right), \quad J=\left(\begin{array}{ll}
0_{s \times(N-s)} & I_{s \times s}
\end{array}\right) .
$$

Observe that invertibility of $G_{d}$ follows from that of $\bar{A}_{d}^{11}$ and $\bar{B}_{d, d}^{22}$. Thus, the system (3.178) can be rewritten

$$
\partial_{z} U-G_{d}^{-1} M U=G_{d}^{-1} F .
$$

It is perhaps not immediately obvious that the matrices $\left(G_{d}^{-1} M\right)_{ \pm}$coincide with $G_{ \pm}(q, \zeta)$ as in (3.163), but a short computation confirms this:

$$
\left(G_{d}^{-1} M\right)_{ \pm}(q, \zeta)=G_{ \pm}(q, \zeta)
$$


Proposition 3.43 (Low frequency). - Let $q \in \mathcal{Q}$. There is a $C^{\infty}$ invertible matrix $\mathcal{V}_{+}(q, \zeta)$ defined on a neighborhood $\Omega$ of $(\underline{q}, 0)$ such that

$$
\mathcal{V}_{+}^{-1} G_{+} \mathcal{V}_{+}=\left(\begin{array}{cc}
H_{+} & 0 \\
0 & P_{+}
\end{array}\right):=G_{B,+} \text { on } \Omega,
$$

with $H_{+}(q, \zeta)$ of dimension $N \times N$ and $P_{+}(q, \zeta)$ of dimension $s \times s$. In fact we have:

(a) $P_{+}(q, \zeta)=P_{0}^{+}(q)+\mathrm{O}(|\zeta|)$ for $P_{0}^{+}$as in (3.173); in particular, the eigenvalues of $P_{+}$ satisfy $|\Re \mu| \geqslant c>0$ on $\Omega$.

(b) $H_{+}(q, \zeta)=H_{0}^{+}(q, \zeta)+\mathrm{O}\left(|\zeta|^{2}\right)$, where

$$
H_{0}^{+}(q, \zeta)=-\left(\bar{A}_{d}\right)_{+}^{-1}\left((i \tau+\gamma) I+\sum_{j=1}^{d-1} i \eta_{j} \bar{A}_{j}(q)\right)_{+} .
$$

(c) At $\zeta=0 \mathcal{V}_{+}$has the triangular form

$$
\mathcal{V}_{+}(q, 0)=\left(\begin{array}{cc}
I_{N \times N} & \bar{V}_{+} \\
0 & I_{s \times s}
\end{array}\right) .
$$

The same result holds with '+' replaced by '-'.

Proof. - 1. The spectral separation described in Lemma 3.40 and the fact that 0 is a semisimple eigenvalue of multiplicity $N$ imply that there is a smooth matrix $\mathcal{V}_{+}$defined on a neighborhood of $(q, 0)$ such that $(3.182)$ holds with $H_{+}(q, 0)=0$ and $P_{+}(q, 0)$ invertible with no eigenvalue on the imaginary axis.

The span of the first $N$ columns of $\mathcal{V}_{+}, \operatorname{span}\left(\mathcal{V}_{+, I}\right)$, is an invariant subspace for $G_{+}$, and at $\zeta=0$ this space is

$$
\operatorname{ker} G_{+}(q, 0)=\mathbb{C}^{N} \times\{0\}^{s} .
$$

Thus, performing a smooth change of basis in $\operatorname{span}\left(\mathcal{V}_{+, I}\right)$, we can arrange so that the first $N$ columns of $\mathcal{V}_{+}$have the form

$$
\mathcal{V}_{+, I}(q, \zeta)=\left(\begin{array}{c}
I_{N \times N} \\
W_{+}(q, \zeta)
\end{array}\right)
$$

with $W_{+}$of size $s \times N$ and vanishing at $\zeta=0$.

The span of the last $s$ columns of $\mathcal{V}_{+}, \operatorname{span}\left(\mathcal{V}_{+, I I}\right)$, is also an invariant subspace for $G_{+}$. At $\zeta=0$ we must have

$$
\mathcal{V}_{+, I I}(q, 0)=\left(\begin{array}{l}
\mathcal{V}_{a} \\
\mathcal{V}_{b}
\end{array}\right)
$$

where the columns of the $s \times s$ matrix $\mathcal{V}_{b}$ span $\mathbb{C}^{s}$. Thus, performing a smooth change of basis in $\operatorname{span}\left(\mathcal{V}_{+, I I}\right)$, we can arrange to have

$$
\mathcal{V}_{+, I I}(q, 0)=\left(\begin{array}{c}
\bar{V}_{+} \\
I_{s \times s}
\end{array}\right) .
$$

This proves (3.184).

2. Writing $G_{+} \mathcal{V}_{+}=\mathcal{V}_{+} G_{B,+}$ at $\zeta=0$ using (3.172) and (3.184), we find that the lower right block of $G_{B,+}$ at $\zeta=0$ is $P_{0}^{+}(q)$ as in (3.173).

3. Here we use the notation (3.177)-(3.179) and suppress ' + ' subscripts. By (3.182) $G \mathcal{V}_{I}=$ $\mathcal{V}_{I} H$, hence $M \mathcal{V}_{I}=G_{d} \mathcal{V}_{I} H$ and equating components gives

$4^{\mathrm{e}}$ SÉRIE - TOME $39-2006-\mathrm{N}^{\circ} 1$ 


$$
\mathcal{M}=-\mathcal{A} H+\bar{B}_{d} W H .
$$

Taking the first order term in (3.189) at $\zeta=0$ gives (3.183).

4. The argument for $G_{-}$is the same.

Remark 3.44. - One can just as easily read off an explicit second order term for $H$ from (3.189), but we shall not need that here.

COROLLARY 3.45. - Let $s_{ \pm}$be the number of eigenvalues $\mu$ of $P_{0}^{ \pm}(q)$ (3.173) with $\mp \Re \mu>0$, respectively. For $N_{ \pm}$as in (H6) we have

$$
s_{+}+s_{-}-s=N_{+}+N_{-}-N
$$

Proof. - Using (3.182), (3.183) and taking $\zeta=(0, \gamma, 0)$ with $\gamma>0$ small, we see that the number of eigenvalues of $G_{+}$in $\Re \mu<0$ is $s_{+}+\left(N-N_{+}\right)$. By Lemma 3.39

$$
s_{+}+\left(N-N_{+}\right)=s+H .
$$

Similarly, $s_{-}+\left(N-N_{-}\right)=N-H$. These equations imply (3.190).

Remark 3.46. - The above proof and properties of $\mathcal{W}_{ \pm}, \mathcal{V}_{ \pm}$imply that for $\mathbb{F}^{ \pm}(q, \zeta)$ (3.170) and $\mathbb{E}^{ \pm}(q, \zeta)$ (3.171), we have

$$
\operatorname{dim} \mathbb{E}^{ \pm}(q, \zeta)=\operatorname{dim} \mathbb{F}^{ \pm}(q, \zeta)=s_{ \pm}+\left(N-N_{ \pm}\right)
$$

\subsubsection{Generalized block structure, low frequency symmetrizers, estimates}

From this point on arguments from earlier papers go through with no essential changes to prove the frozen coefficient, low frequency estimate (3.157).

One first performs another conjugation of $G_{B, \pm}$ as in (3.182) to generalized block structure using the argument of [30, Appendix A.1]. The hypothesis (H4) of hyperbolicity with constant multiplicity is used here. Next, the construction of [30, Appendix A.3], yields symmetrizers for the modified low frequency problem (3.153). Finally, the argument of [12, Appendix A.1] shows how those symmetrizers imply the estimate (3.157).

\section{Variable coefficient $L^{2}$ estimates}

\subsection{Regularity assumptions and results}

In this section we prove estimates for the variable coefficient transmission problem corresponding to (3.3):

$$
\begin{gathered}
\mathcal{L}\left(z, q, \zeta, \partial_{z}\right) u-\psi \mathcal{K}(z, q, \zeta)=f \\
{[u]=0, \quad\left[\partial_{z} u_{2}\right]=0, \quad a(\zeta) \psi+\ell(q) \cdot u_{2}^{+}(0)=0 \quad \text { on } z=0,}
\end{gathered}
$$

where $\mathcal{L}$ has been modified in accordance with Remark 3.32. Our effort in the first few subsections will concern the case where $u$ and $f$ are supported near the front.

Setting $D=\frac{1}{i} \partial$ we consider

$$
\begin{aligned}
& \frac{1}{\varepsilon} \mathcal{L}\left(\frac{x}{\varepsilon}, q^{\varepsilon}, \varepsilon D_{t}, \varepsilon \gamma, \varepsilon D_{y}, \varepsilon \partial_{x}\right) u-\frac{1}{\varepsilon^{2}} \mathcal{K}\left(\frac{x}{\varepsilon}, q^{\varepsilon}, \varepsilon D_{t}, \varepsilon \gamma, \varepsilon D_{y}\right) \psi=f, \\
& {[u]=0, \quad\left[\partial_{x} u_{2}\right]=0, \quad\left(\partial_{t}+\gamma\right) \psi-\varepsilon \triangle_{y} \psi+\ell\left(q^{\varepsilon}\right) \cdot u_{2}^{+}=0 \quad \text { on } x=0,}
\end{aligned}
$$

where 


$$
\begin{aligned}
q^{\varepsilon}(t, y, x) & =\left(p(t, y), u_{\varepsilon}^{\prime}(t, y, x, \varepsilon), h_{\varepsilon}^{\prime}(t, y), r_{\varepsilon}(t, y, x)\right) \quad \text { with } \\
p(t, y) & =\left(u_{+}^{0}(t, y, 0), u_{-}^{0}(t, y, 0), d \psi^{0}(t, y)\right) \in \mathcal{C},
\end{aligned}
$$

$u_{\varepsilon}^{\prime}, h_{\varepsilon}^{\prime}$ represent perturbations of $u_{ \pm}^{0}$ and $d \psi^{0}$ respectively, and $r_{\varepsilon}$ is a perturbation of $\mathbb{A}_{d}^{21}$ which we turn out to non-negligible only in the high frequency estimate. When $\left(p, u_{\varepsilon}^{\prime}, h_{\varepsilon}^{\prime}\right)$ is constant and $r_{\varepsilon} \equiv 0$, the transmission problem (4.2) is the same as (2.57), (2.59). The principal part of the linearized operator that we use in the nonlinear iteration scheme turns out to have precisely the form of the operator in (4.2).

For the purposes of the $L^{2}$ estimate we make the following regularity assumptions, all of which are satisfied in the later application to the nonlinear iteration scheme. Set

$$
W^{k, \infty}\left(\mathbb{R}^{d}\right)=\left\{v(t, y): \partial_{t, y}^{\alpha} v \in L^{\infty} \text { for }|\alpha| \leqslant k\right\}
$$

and define $W^{k, \infty}\left(\mathbb{R}_{ \pm}^{d+1}\right)$ similarly.

ASSUMPTION 4.1 (H10). -

(a) $p(t, y) \in \mathcal{C}$; the functions $u_{ \pm}^{0}(t, y, x)$ are $C^{2}$ up to the boundary, take values in a compact subset of $\mathcal{U}$, and belong to $W^{2, \infty}\left(\mathbb{R}_{ \pm}^{d+1}\right) ; \psi^{0} \in W^{3, \infty}\left(\mathbb{R}^{d}\right)$.

There exists an $\varepsilon_{0}>0$ such that for $\varepsilon \in\left(0, \varepsilon_{0}\right]$ :

(b) The families $\left\{u_{\varepsilon}^{\prime \pm}\right\},\left\{\nabla_{t, y, x} u_{\varepsilon}^{\prime \pm}\right\},\left\{\varepsilon \partial_{x}^{2} u_{\varepsilon}^{\prime \pm}, \nabla_{t, y}\left(\nabla_{t, y, x} u_{\varepsilon}^{\prime \pm}\right)\right\}$ are bounded in $L^{\infty}\left(\mathbb{R}_{ \pm}^{d+1}\right)$.

(c) The traces $\left.u_{\varepsilon}^{\prime \pm}\right|_{x=0}$ are $\mathrm{O}(\varepsilon)$ in $W^{1, \infty}\left(\mathbb{R}^{d}\right)$ and satisfy $\left.u_{\varepsilon}^{\prime+}\right|_{x=0}=\left.u_{\varepsilon}^{\prime-}\right|_{x=0}$.

(d) The family $\left\{\frac{h_{\varepsilon}^{\prime}}{\varepsilon}\right\}$ is bounded in $W^{2, \infty}\left(\mathbb{R}^{d}\right)$.

(e) The families $\left\{\frac{r_{\varepsilon}}{\varepsilon}, \nabla_{t, y}\left(\frac{r_{\varepsilon}}{\varepsilon}\right), \nabla_{t, y}^{2}\left(\frac{r_{\varepsilon}}{\varepsilon}\right)\right\}$ are bounded in $L^{\infty}\left(\mathbb{R}_{ \pm}^{d+1}\right)$.

(f) For all $(t, y, x) \in \mathbb{R}^{d+1}$ and $q^{\varepsilon}$ as in (4.3), we have $W\left(\frac{x}{\varepsilon}, q^{\varepsilon}\right) \in \mathcal{U}^{*}$. There exists $Z>0$ such that for $\left|\frac{x}{\varepsilon}\right|>Z, W\left(\frac{x}{\varepsilon}, q^{\varepsilon}\right) \in \mathcal{U}$.

The main step in the proof of Theorem 1.1 is a weighted $L^{2}$ estimate. Define weights

$$
\begin{gathered}
\Lambda_{\varepsilon}(\zeta)=\Lambda(\varepsilon \zeta)=\left(1+(\varepsilon \tau)^{2}+(\varepsilon \gamma)^{2}+|\varepsilon \eta|^{4}\right)^{1 / 4}, \\
\lambda_{\varepsilon}(\zeta)=\left\{\begin{array}{lc}
\left(\gamma+\varepsilon|\zeta|^{2}\right)^{1 / 2}, & \text { when }|\varepsilon \zeta| \leqslant 1, \\
\frac{1}{\sqrt{\varepsilon}}, & \text { when } \varepsilon|\zeta| \geqslant 1,
\end{array}\right.
\end{gathered}
$$

and

$$
\mu_{\varepsilon}(\zeta)= \begin{cases}|\zeta| \lambda_{\varepsilon}, & \text { when }|\varepsilon \zeta| \leqslant 1, \\ (\Lambda / \varepsilon)^{3 / 2}, & \text { when }|\varepsilon \zeta| \geqslant 1 .\end{cases}
$$

Observe that the expressions defining $\lambda_{\varepsilon}$ in the two frequency regimes are of the same order when $|\varepsilon \zeta| \approx 1$, and similarly for $\mu_{\varepsilon}$. Moreover, on any set of frequencies such that $0 \leqslant|\varepsilon \zeta| \leqslant R$, we have $1 \leqslant \Lambda_{\varepsilon} \leqslant C_{R}$.

Given a weight function $\phi(\zeta)$ we use the notation

$$
|u|_{\phi}=\left(\int_{\mathbb{R}^{d}} \phi(\tau, \gamma, \eta)^{2}|\hat{u}(\tau, \eta)|^{2} d \tau d \eta\right)^{1 / 2} .
$$

When $u^{ \pm}$also depends on $x$, we set

$$
\begin{aligned}
\left\|u^{ \pm}\right\|_{\phi} & =\left(\int_{ \pm x \geqslant 0}\left|u^{ \pm}(\cdot, x)\right|_{\phi}^{2} d x\right)^{1 / 2}, \\
\|u\|_{\phi} & =\left\|u^{+}\right\|_{\phi}+\left\|u^{-}\right\|_{\phi} .
\end{aligned}
$$

When $\phi=1$ we write $|u|$ or $\|u\|$.

$4^{\mathrm{e}}$ SÉRIE - TOME $39-2006-\mathrm{N}^{\circ} 1$ 
Theorem 4.2 ( $L^{2}$ estimate). - Assume (H0)-(H10) and fix $\kappa_{0}>0$. Suppose $\left(u^{ \pm}, \psi\right)$ is a solution of the transmission problem (4.2), where $u^{ \pm}, f^{ \pm}$are $C^{\infty}$ with compact support on $\overline{\mathbb{R}}_{ \pm}^{d+1} \cap\left\{|x| \leqslant 2 \kappa_{0}\right\}$ and $\psi \in C_{0}^{\infty}\left(\mathbb{R}^{d}\right)$. For $\kappa_{0}$ small enough, there exist positive constants $C$, $\gamma_{0}$, and $\varepsilon_{0}$ independent of $(u, \psi)$, such that for $\gamma$ and $\varepsilon$ satisfying

$$
\gamma \geqslant \gamma_{0}, \quad \varepsilon \in\left(0, \varepsilon_{0}\right]
$$

there holds

$$
\begin{aligned}
& \left\|u_{1}\right\|_{\lambda_{\varepsilon}^{2}}+\left\|u_{2}\right\|_{\lambda_{\varepsilon}^{2} \Lambda_{\varepsilon}}+\sqrt{\varepsilon}\left\|\partial_{x} u_{2}\right\|_{\lambda_{\varepsilon}}+\left|u_{1}(0)\right|_{\lambda_{\varepsilon}}+\left|u_{2}(0)\right|_{\lambda_{\varepsilon} \Lambda_{\varepsilon}^{1 / 2}} \\
& \quad+\varepsilon\left|\partial_{x} u_{2}(0)\right|_{\lambda_{\varepsilon} \Lambda_{\varepsilon}^{-1 / 2}}+|\psi|_{\mu_{\varepsilon} \Lambda_{\varepsilon}} \\
& \leqslant C\left(\left\|f_{1}\right\|+\left\|f_{2}\right\|_{\Lambda_{\varepsilon}^{-1}}\right) .
\end{aligned}
$$

The proof of Theorem 4.2 will occupy most of Section 4 . First we make an easy reduction by introducing a cutoff supported near the front. For any $\kappa_{0}>0$ choose $\kappa \in C_{0}^{\infty}\left(\left(-\kappa_{0}, \kappa_{0}\right)\right)$ and equal to one for $|x| \leqslant \frac{\kappa_{0}}{2}$. Since all the coefficients of $\mathcal{K}$ involve at least one derivative in $z$ of $W$, they are exponentially decaying in $\frac{x}{\varepsilon}$. Thus, we have

$$
\begin{gathered}
\frac{1}{\varepsilon^{2}}(1-\kappa(x)) \mathcal{K}\left(\frac{x}{\varepsilon}, q^{\varepsilon}, \varepsilon D_{t}, \varepsilon \gamma, \varepsilon D_{y}\right) \psi:=\left(\begin{array}{c}
k_{1}^{\varepsilon} \\
k_{2}^{\varepsilon}
\end{array}\right) \text { with } \\
\left\|k_{1}^{\varepsilon}\right\|+\left\|k_{2}^{\varepsilon}\right\|_{\Lambda_{\varepsilon}^{-1}} \leqslant C_{L} \varepsilon^{L}\left|\nabla_{\gamma} \psi\right|
\end{gathered}
$$

for any $L>0$. Here

$$
\nabla_{\gamma} \psi=\left(\left(\partial_{t}+\gamma\right) \psi, \partial_{1} \psi, \ldots, \partial_{d-1} \psi\right)
$$

and we have used the fact that only $k_{2}^{\varepsilon}$ involves two derivatives of $\psi$. (4.12) implies that the estimate of Theorem 4.2 follows immediately from the same estimate for the modified transmission problem:

$$
\begin{aligned}
& \frac{1}{\varepsilon} \mathcal{L}\left(\frac{x}{\varepsilon}, q^{\varepsilon}, \varepsilon D_{t}, \varepsilon \gamma, \varepsilon D_{y}, \varepsilon \partial_{x}\right) u-\frac{1}{\varepsilon^{2}} \kappa(x) \mathcal{K}\left(\frac{x}{\varepsilon}, q^{\varepsilon}, \varepsilon D_{t}, \varepsilon \gamma, \varepsilon D_{y}\right) \psi=f, \\
& {[u]=0, \quad\left[\partial_{x} u_{2}\right]=0, \quad\left(\partial_{t}+\gamma\right) \psi-\varepsilon \triangle_{y} \psi+\ell\left(q^{\varepsilon}\right) \cdot u_{2}^{+}=0 \quad \text { on } x=0 .}
\end{aligned}
$$

THEOREM 4.3 (Estimate near the front). - Assume (H0)-(H10) and fix $\kappa_{0}>0$. Assume $u^{ \pm}$, $f^{ \pm}$are $C^{\infty}$ with compact support on $\overline{\mathbb{R}}_{ \pm}^{d+1} \cap\left\{|x| \leqslant 2 \kappa_{0}\right\}, \psi \in C_{0}^{\infty}\left(\mathbb{R}^{d}\right)$, and $(u, \psi)$ satisfies (4.14). Then for $\kappa_{0}$ small enough, there exist $\gamma_{0}$, $\varepsilon_{0}$ such that the estimate (4.11) holds for $\gamma \geqslant \gamma_{0}$, $0<\varepsilon \leqslant \varepsilon_{0}$.

Theorem 4.3 is a direct corollary of the next theorem. For positive constants $\rho_{0}<R$ to be chosen, let $\chi_{L}(\zeta), \chi_{M}(\zeta), \chi_{H}(\zeta)$ be low, medium, and high frequency cutoffs in $C^{\infty}\left(\mathbb{R}^{d+1}\right)$ such that

$$
\begin{aligned}
& \chi_{L}+\chi_{M}+\chi_{H}=1 \quad \text { on } \mathbb{R}^{d+1}, \\
& \operatorname{supp} \chi_{L} \subset\left\{|\zeta| \leqslant \rho_{0}\right\}, \quad \operatorname{supp} \chi_{H} \subset\{|\zeta| \geqslant R\},
\end{aligned}
$$

and define the semiclassical multiplier

$$
T_{\chi L}^{\varepsilon, \gamma} u=\int e^{i t \tau+i y \eta} \chi_{L}(\varepsilon \zeta) \hat{u}(\tau, \eta, x) d \tau d \eta
$$

(usually we will suppress the superscripts $\varepsilon, \gamma$ on $T_{\chi_{L}}$ ). For $\delta>0$ as in Lemma 3.14, let $\beta_{i}(\zeta) \in \Gamma^{0}, i=1,2$, be chosen so that 


$$
\begin{gathered}
\operatorname{supp} \beta_{1} \subset\left\{0 \leqslant \gamma \leqslant \frac{3 \delta}{4}|\zeta|,|\zeta| \geqslant 1\right\}, \quad \operatorname{supp} \beta_{2} \subset\left\{\frac{\delta}{2}|\zeta| \leqslant \gamma,|\zeta| \geqslant 1\right\}, \\
\beta_{1}=1 \quad \text { on }\left\{0 \leqslant \gamma \leqslant \frac{\delta}{2}|\zeta|,|\zeta| \geqslant 2\right\}, \quad \beta_{2}=1 \quad \text { on }\left\{\frac{3 \delta}{4}|\zeta| \leqslant \gamma,|\zeta| \geqslant 2\right\}, \\
\beta_{1}+\beta_{2}=1 \quad \text { on }|\zeta| \geqslant 2 .
\end{gathered}
$$

Thus, in particular we have

$$
\beta_{1} \chi_{H}+\beta_{2} \chi_{H}=\chi_{H} \text { for all } \zeta .
$$

THEOREM 4.4 (Estimates near the front by frequency size). - Assume (H0)-(H10). Suppose $\left(u^{ \pm}, \psi\right)$ is a solution of the transmission problem (4.14), where $u^{ \pm}, f^{ \pm}$are $C^{\infty}$ with compact support on $\overline{\mathbb{R}}_{ \pm}^{d+1} \cap\left\{|x| \leqslant 2 \kappa_{0}\right\}$, and $\psi \in C_{0}^{\infty}\left(\mathbb{R}^{d}\right)$. If $\kappa_{0}$ is small enough, then provided $\rho_{0}$ (respectively $R$ ) in (4.15) is small (respectively large) enough, there exist positive constants $C$ and $\varepsilon_{0}$ independent of $(u, \psi)$ such that for $\gamma \geqslant 1$ and $\varepsilon \in\left(0, \varepsilon_{0}\right]$ there holds:

$$
\begin{aligned}
& \left\|T_{\chi_{L}} u\right\|_{\lambda_{\varepsilon}^{2}}+\sqrt{\varepsilon}\left\|\partial_{x} T_{\chi_{L}} u_{2}\right\|_{\lambda_{\varepsilon}}+\left|T_{\chi_{L}} u(0)\right|_{\lambda_{\varepsilon}}+\varepsilon\left|\partial_{x} T_{\chi_{L}} u_{2}(0)\right|_{\lambda_{\varepsilon}}+\left|T_{\chi_{L}} \psi\right|_{|\zeta| \lambda_{\varepsilon}} \\
& \leqslant C\left(\left\|f_{1}\right\|+\left\|f_{2}\right\|+\text { a.t. }\right), \\
& \frac{1}{\varepsilon}\left\|T_{\chi_{M}} u\right\|+\left\|\partial_{x} T_{\chi_{M}} u_{2}\right\|+\frac{1}{\sqrt{\varepsilon}}\left|T_{\chi_{M}} u(0)\right|+\sqrt{\varepsilon}\left|\partial_{x} T_{\chi_{M}} u_{2}(0)\right|+\frac{1}{\varepsilon^{3 / 2}}\left|T_{\chi_{M}} \psi\right| \\
& \leqslant C\left(\left\|f_{1}\right\|+\left\|f_{2}\right\|+\text { a.t. }\right), \\
& \text { (4.21) }\left(\frac{1}{\varepsilon}+\gamma\right)\left\|T_{\beta_{1} \chi_{H}} u_{1}\right\|+\frac{1}{\varepsilon}\left\|T_{\beta_{1} \chi_{H}} u_{2}\right\|_{\Lambda_{\varepsilon}}+\left\|\partial_{x} T_{\beta_{1} \chi_{H}} u_{2}\right\|+\left(\frac{1}{\varepsilon}+\gamma\right)^{1 / 2}\left|T_{\beta_{1} \chi_{H}} u_{1}(0)\right| \\
& +\frac{1}{\sqrt{\varepsilon}}\left|T_{\beta_{1} \chi_{H}} u_{2}(0)\right|_{\Lambda_{\varepsilon}^{1 / 2}}+\sqrt{\varepsilon}\left|\partial_{x} T_{\beta_{1} \chi_{H}} u_{2}(0)\right|_{\Lambda_{\varepsilon}^{-1 / 2}}+\frac{1}{\varepsilon^{3 / 2}}\left|T_{\beta_{1} \chi_{H}} \psi\right|_{\Lambda_{\varepsilon}^{5 / 2}} \\
& \leqslant C\left(\left\|f_{1}\right\|+\left\|f_{2}\right\|_{\Lambda_{\varepsilon}^{-1}}+\left\|u_{1}\right\|+\left\|u_{2}\right\|+\varepsilon\left\|\partial_{x} u_{2}\right\|+|\psi|_{|\zeta|}+\text { a.t. }\right), \\
& \left\|T_{\beta_{2} \chi_{H}} u_{1}\right\|_{|\zeta|}+\frac{1}{\varepsilon}\left\|T_{\beta_{2} \chi_{H}} u_{2}\right\|_{\Lambda_{\varepsilon}}+\left\|\partial_{x} T_{\beta_{2} \chi_{H}} u_{2}\right\|+\left|T_{\beta_{2} \chi_{H}} u_{1}(0)\right|_{|\zeta|^{1 / 2}} \\
& +\frac{1}{\sqrt{\varepsilon}}\left|T_{\beta_{2} \chi_{H}} u_{2}(0)\right|_{\Lambda_{\varepsilon}^{1 / 2}}+\sqrt{\varepsilon}\left|\partial_{x} T_{\beta_{2} \chi_{H}} u_{2}(0)\right|_{\Lambda_{\varepsilon}^{-1 / 2}}+\frac{1}{\varepsilon^{3 / 2}}\left|T_{\beta_{2} \chi_{H}} \psi\right|_{\Lambda_{\varepsilon}^{5 / 2}} \\
& \leqslant C\left(\left\|f_{1}\right\|+\left\|f_{2}\right\|_{\Lambda_{\varepsilon}^{-1}}+\text { a.t. }\right) \text {. }
\end{aligned}
$$

Here a.t. stands for a finite sum of "absorbable terms", terms that can be absorbed by the sum of the left sides of (4.19)-(4.22) by taking $\gamma$ large and $\varepsilon$ small. Four such terms are written out in (4.21).

The low frequency estimate does not use $(\mathrm{H} 3)$; the medium frequency estimate does not use (H3), (H4); and the high frequency estimate does not use (H4), (H9).

Proof of Theorem 4.3 assuming Theorem 4.4. - 1. Add the low (LF), medium (MF), and high frequency (HF) estimates and absorb a.t. terms by taking $\gamma_{0}$ large enough and $\varepsilon_{0}$ small enough to show that the sum of the left sides of (4.19)-(4.22) is $\leqslant$

$$
C\left(\left\|f_{1}\right\|+\left\|f_{2}\right\|_{\Lambda_{\varepsilon}^{-1}}\right) .
$$

2. Use (4.15), (4.18), and the fact that

$$
\begin{aligned}
& \lambda_{\varepsilon} \approx \frac{1}{\sqrt{\varepsilon}} \text { on MF, HF, } \\
& \Lambda_{\varepsilon} \approx 1 \quad \text { on LF, MF }
\end{aligned}
$$

to see that the left side of (4.11) is $\leqslant C\left(\left\|f_{1}\right\|+\left\|f_{2}\right\|_{\Lambda_{\varepsilon}^{-1}}\right)$. 
Remark 4.5. - We have stated the estimates (4.19)-(4.22) for the transmission problem (4.14) with homogeneous transmission conditions, but the proofs automatically handle the nonhomogeneous case as well. Suppose that in (4.14) we take

$$
[u]=\left(\begin{array}{l}
k_{1} \\
k_{2}
\end{array}\right), \quad\left[\partial_{x} u_{2}\right]=k_{3}, \quad\left(\partial_{t}+\gamma\right) u-\varepsilon \triangle_{y} u+\ell\left(q^{\varepsilon}\right) \cdot u=k_{4} \quad \text { on } x=0 .
$$

Then in the estimates (4.19)-(4.22) we just need to include boundary norms of the $k_{i}$ on the right. In each estimate the weight on $k_{1}$ is the same as the one that appears on the left side on $\left|u_{1}(0)\right|$, the weight on $k_{2}$ is the one on $\left|u_{2}(0)\right|$, the weight on $k_{3}$ is the one on $\left|\partial_{x} u_{2}(0)\right|$, and the weight on $k_{4}$ is the one on $\left|u_{2}(0)\right|$. The same comment applies to the composite estimate (4.11). For example, the version of (4.11) with $k_{4} \neq 0$ has the term

$$
\left|k_{4}\right|_{\lambda_{\varepsilon} \Lambda_{\varepsilon}^{1 / 2}}
$$

on the right.

\subsubsection{First order system}

Set $U=\left(u_{1}, u_{2}, \varepsilon \partial_{x} u_{2}\right)=\left(u_{1}, u_{2}, u_{3}\right)$. With $\mathcal{J}$ as in (3.33) and $G(z, q, \zeta)$ as in (3.34), we may rewrite (4.14) as a first order system:

$$
\begin{aligned}
& \partial_{x} U-\frac{1}{\varepsilon} G\left(\frac{x}{\varepsilon}, q^{\varepsilon}, \varepsilon D_{t}, \varepsilon \gamma, \varepsilon D_{y}\right) U \\
& \quad=\mathcal{J}\left(\frac{x}{\varepsilon}, q^{\varepsilon}\right)\left(f+\frac{1}{\varepsilon^{2}} \kappa(x) \mathcal{K}\left(\frac{x}{\varepsilon}, q^{\varepsilon}, \varepsilon D_{t}, \varepsilon \gamma, \varepsilon D_{y}\right) \psi\right) \\
& {[U]=0, \quad\left(\partial_{t}+\gamma\right) \psi-\varepsilon \triangle_{y} \psi+\ell\left(q^{\varepsilon}\right) \cdot u_{2}=0 \quad \text { on } x=0 . }
\end{aligned}
$$

Here

$$
\begin{aligned}
& \frac{1}{\varepsilon^{2}} \kappa \mathcal{K} \psi=\frac{1}{\varepsilon} m^{\varepsilon}\left(t, y, x, \varepsilon D_{t}, \varepsilon \gamma, \varepsilon D_{y}\right) \nabla_{\gamma} \psi, \quad \text { where } \\
& m^{\varepsilon} \nabla_{\gamma} \psi=m_{0}^{\varepsilon}(t, y, x)\left(\partial_{t}+\gamma\right) \psi+\sum_{j=1}^{d-1} m_{j}^{\varepsilon}\left(t, y, x, \varepsilon D_{t}, \varepsilon \gamma, \varepsilon D_{y}\right) \partial_{j} \psi
\end{aligned}
$$

and

$$
\begin{aligned}
m_{0}^{\varepsilon}(t, y, x) & :=\kappa \tilde{m}_{0}\left(\frac{x}{\varepsilon}, q^{\varepsilon}\right), \quad \tilde{m}_{0}(z, q):=\partial_{z} f_{0}(W(z, q)), \\
m_{j}^{\varepsilon}(t, y, x, \zeta) & :=\kappa \tilde{m}_{j}\left(\frac{x}{\varepsilon}, q^{\varepsilon}\right), \\
\tilde{m}_{j}(z, q) & :=\left(\partial_{z} f_{j}-\partial_{z}\left(\left(\widetilde{B}_{j, d}+\widetilde{B}_{d, j}\right) \partial_{z} W\right)-\sum_{k=1}^{d-1} B_{j, k} \partial_{z} W i \eta_{k}\right)
\end{aligned}
$$

(recall $\left.\widetilde{B}_{j, d}=\widetilde{B}_{j, d}\left(W(z, q), \nu\left(h+h^{\prime}\right)\right)\right)$. Observe that

$$
\mathcal{K}(z, q, \zeta)=\tilde{m}_{0}(z, q)(i \tau+\gamma)+\sum_{j=1}^{d-1} \tilde{m}_{j}(z, q, \zeta) i \eta_{j} .
$$

Similarly, we set 


$$
\begin{aligned}
j^{\varepsilon}(t, y, x) & =\mathcal{J}\left(\frac{x}{\varepsilon}, q^{\varepsilon}\right), \\
g^{\varepsilon}(t, y, x, \zeta) & =G\left(\frac{x}{\varepsilon}, q^{\varepsilon}, \zeta\right), \\
g^{i j}(t, y, x, \zeta) & =G^{i j}\left(\frac{x}{\varepsilon}, q^{\varepsilon}, \zeta\right),
\end{aligned}
$$

where the $G^{i j}$ are the entries of $G$ (3.36).

Thus (4.27) becomes

$$
\begin{gathered}
\partial_{x} U-\frac{1}{\varepsilon} g^{\varepsilon} U=j^{\varepsilon}\left(f+\frac{1}{\varepsilon} m^{\varepsilon} \nabla_{\gamma} \psi\right), \\
{[U]=0, \quad\left(\partial_{t}+\gamma\right) \psi-\varepsilon \triangle_{y} \psi+\ell\left(q^{\varepsilon}\right) \cdot u_{2}=0 \quad \text { on } x=0 .}
\end{gathered}
$$

Remark 4.6. - Depending on the context, we use expressions like $g^{\varepsilon}$ or $m^{\varepsilon}$ sometimes to denote a symbol, and sometimes to denote the associated semiclassical operator.

Using the definition of $\mathcal{J}$ (3.33), we define $h_{i}$ and $n_{i}^{\varepsilon}, i=1,2$, by

$$
j^{\varepsilon} f=\left(\begin{array}{c}
h_{1} \\
0 \\
h_{2}
\end{array}\right), \quad j^{\varepsilon} m^{\varepsilon}=\left(\begin{array}{c}
n_{1}^{\varepsilon} \\
0 \\
n_{2}^{\varepsilon}
\end{array}\right)
$$

and rewrite the system (4.32) as

$$
\begin{gathered}
\partial_{x} U-\frac{1}{\varepsilon} g^{\varepsilon} U=\left(\begin{array}{c}
h_{1} \\
0 \\
h_{2}
\end{array}\right)+\frac{1}{\varepsilon}\left(\begin{array}{c}
n_{1}^{\varepsilon} \nabla_{\gamma} \psi \\
0 \\
n_{2}^{\varepsilon} \nabla_{\gamma} \psi
\end{array}\right), \\
{[U]=0, \quad\left(\partial_{t}+\gamma\right) \psi-\varepsilon \triangle_{y} \psi+\ell\left(q^{\varepsilon}\right) \cdot u_{2}=0 \quad \text { on } x=0 .}
\end{gathered}
$$

Remark 4.7. - (1) To prove Theorem 4.4 it clearly suffices to prove the estimates (4.19)(4.22) for the system (4.34), with $\left(h_{1}, h_{2}\right)$ in place of $\left(f_{1}, f_{2}\right)$. Note that the coefficients of $n_{i}^{\varepsilon}$ are exponentially decreasing in $\frac{x}{\varepsilon}$. Also, $n_{1}^{\varepsilon}$ is of order zero, while $n_{2}^{\varepsilon}$ is of order one with $\partial_{y_{k}}$ derivatives only.

(2) A partition of unity allows us to assume that the support of $(U, \psi)$ is as close as we like to any prescribed basepoint $(\underline{t}, \underline{y}, 0)$. It is easy to check that smooth cutoffs $\phi(t, y, x)$ introduce only absorbable errors (a.t. terms in the sense of Theorem 4.4).

\subsection{Semiclassical paradifferential calculi}

We collect here the facts about the paradifferential calculi that we will use in the variable coefficient estimates. We refer to Appendix B of [30] for detailed proofs. We shall use both homogeneous and parabolic calculi. With each calculus there is associated a scale of Sobolev spaces on which the operators naturally act.

First, we introduce homogeneous and parabolic weights. With $\zeta=(\tau, \gamma, \eta)$ as before and $\alpha=\left(\alpha_{\tau}, \alpha_{\eta}\right) \in \mathbb{N} \times \mathbb{N}^{d-1}$ a multi-index, set $\mathbb{R}_{+}^{d+1}=\{\zeta: \gamma \geqslant 0\}$ and

$$
\begin{gathered}
\langle\zeta\rangle=\left(1+|\zeta|^{2}\right)^{1 / 2} \\
\Lambda(\zeta)=\left(1+\tau^{2}+\gamma^{2}+|\eta|^{4}\right)^{1 / 4} \\
|\alpha|=\alpha_{\tau}+\left|\alpha_{\eta}\right|, \quad\|\alpha\|=2 \alpha_{\tau}+\left|\alpha_{\eta}\right| .
\end{gathered}
$$


DEFINITION 4.8 (Symbols).-

(1) Let $\mu \in \mathbb{R}$. The space of homogeneous symbols $\Gamma_{0}^{\mu}$ is the set of locally $L^{\infty}$ functions $a(t, y, x, \zeta)$ on $\mathbb{R}^{d+1} \times \mathbb{R}_{+}^{d+1}$ which are $C^{\infty}$ in $\zeta$ and satisfy:

$$
\left|\partial_{\tau, \eta}^{\alpha} a\right| \leqslant C_{\alpha}\langle\zeta\rangle^{\mu-|\alpha|}, \quad \text { for all }(t, y, x, \zeta) \text { and } \alpha .
$$

(2) For $k=0,1,2, \ldots, \Gamma_{k}^{\mu}$ denotes the space of symbols $a \in \Gamma_{0}^{\mu}$ such that $\partial_{t, y}^{\alpha} a \in \Gamma_{0}^{\mu}$ for $|\alpha| \leqslant k$.

(3) The spaces of parabolic symbols $P \Gamma_{0}^{\mu}$ and $P \Gamma_{k}^{\mu}$ are defined in the same way, using $\Lambda(\zeta)$ in place of $\langle\zeta\rangle$ and $\|\alpha\|$ in place of $|\alpha|$.

We keep the same notation for the spaces of symbols defined just for $\pm x \geqslant 0$. Observe that symbols in $\Gamma_{k}^{\mu}$ which are independent of $x$ constitute a subspace of $\Gamma_{k}^{\mu}$, and similarly for the spaces $P \Gamma_{k}^{\mu}$.

The spaces $\Gamma_{k}^{\mu}$ are equipped with the natural seminorms

$$
|a|_{\mu, k, N}:=\sup _{|\alpha| \leqslant N} \sup _{|\beta| \leqslant k(t, y, x, \zeta)} \sup _{|\zeta\rangle^{|\alpha|-\mu}\left|\partial_{t, y}^{\beta} \partial_{\zeta}^{\alpha} a(t, y, x, \zeta)\right| .}
$$

The seminorms on the spaces $P \Gamma_{k}^{\mu}$ are defined in the same way by the substitutions described earlier.

We consider a semiclassical quantization of symbols. When $a \in \Gamma_{0}^{\mu}$ is independent of $(t, y)$ the associated homogeneous paradifferential operator acts in $(t, y)$ and is defined by the Fourier multiplier $a(x, \varepsilon \zeta)$ :

$$
T_{a}^{\varepsilon, \gamma} u(t, y, x)=\frac{1}{(2 \pi)^{d}} \int e^{i t \tau+i y \eta} a(x, \varepsilon \zeta) \hat{u}(x, \tau, \eta) d \tau d \eta .
$$

For $a \in P \Gamma_{0}^{\mu}$ the associated parabolic operator $P_{a}^{\varepsilon, \gamma}$ is defined by the same formula. When the symbols depend on $(t, y)$, the corresponding operators are defined by formulas similar to (4.38), except that the symbols are first smoothed in $(t, y)$ using an idea of Bony [2]. The smoothing process in the homogeneous case differs from that in the parabolic case (see [30, Proposition B.7]). When $a(t, y, x, \zeta)$ and $u$ are continuous up to $x=0$, we have

$$
\left.\left(T_{a}^{\varepsilon, \gamma} u\right)\right|_{x=0}=\left.T_{\left.a\right|_{x=0}, \gamma}^{\varepsilon,} u\right|_{x=0}
$$

and similarly for parabolic operators. We use superscripts \pm on $a$ and $u$ to indicate limits at $x=0$ taken in $\pm x \geqslant 0$. We shall often drop the superscripts $\varepsilon, \gamma$ and write the operator defined by (4.38) simply as $T_{a}$.

\subsubsection{Sobolev spaces}

For $s \in \mathbb{R}$ let $H^{s}$ denote the space of functions $u(t, y)$ such that

$$
|u|_{s, \varepsilon, \gamma}:=\left(\int_{\mathbb{R}^{d}}\langle\varepsilon \zeta\rangle^{2 s}|\hat{u}(\tau, \eta)|^{2} d \tau d \eta\right)^{1 / 2}<\infty,
$$

and let $\mathcal{H}^{s}$ be the space of functions $u(t, y, x)$ such that

$$
\|u\|_{s, \varepsilon, \gamma}=\left(\int|u(\cdot, x)|_{s, \varepsilon, \gamma}^{2} d x\right)^{1 / 2}<\infty .
$$

Similarly define spaces $P H^{s}$ and $P \mathcal{H}^{s}$ by substituting the weight $\Lambda(\varepsilon \zeta)$ for $\langle\varepsilon \zeta\rangle$ in (4.40) and (4.41), respectively. We will use the same notation $\|u\|_{s, \varepsilon, \gamma}$ for norms in $\mathcal{H}^{s}$ and $P \mathcal{H}^{s}$. It should be clear from the context which weights are being used. 


\subsubsection{Action on Sobolev spaces, symbolic calculus}

Proposition 4.9 (Action). - For any $a \in \Gamma_{0}^{\mu}$ and $s \in \mathbb{R}$ there is a $C$ such that for $\varepsilon \in(0,1]$, $\gamma \geqslant 1$ and $u \in \mathcal{H}^{s}$ :

$$
\left\|T_{a}^{\varepsilon, \gamma} u\right\|_{s-\mu, \varepsilon, \gamma} \leqslant C\|u\|_{s, \varepsilon, \gamma} .
$$

The constant $C$ is bounded when a remains in a bounded subset of $\Gamma_{0}^{\mu}$.

For $a \in P \Gamma_{0}^{\mu}$ the operators $P_{a}^{\varepsilon, \gamma}$ have the same mapping property on the spaces $P \mathcal{H}^{s}$.

PRoposition 4.10 (Compositions). - Consider $a \in \Gamma_{1}^{\mu}$ and $b \in \Gamma_{1}^{\nu}$. Then $a b \in \Gamma_{1}^{\mu+\nu}$ and there is a $C$ such that for $\varepsilon \in(0,1], \gamma \geqslant 1$ and $u \in \mathcal{H}^{s}$ :

$$
\left\|\left(T_{a}^{\varepsilon, \gamma} \circ T_{b}^{\varepsilon, \gamma}-T_{a b}^{\varepsilon, \gamma}\right) u\right\|_{s-\mu-\nu+1, \varepsilon, \gamma} \leqslant C \varepsilon\|u\|_{s, \varepsilon, \gamma} .
$$

The constant $C$ is bounded when a and $b$ remain in bounded subsets of $\Gamma_{1}^{\mu}$ and $\Gamma_{1}^{\nu}$ respectively. Moreover, if $b$ is independent of $(t, y)$ then $T_{a}^{\varepsilon, \gamma} \circ T_{b}^{\varepsilon, \gamma}=T_{a b}^{\varepsilon, \gamma}$.

The same inequality holds for compositions of operators $P_{a}^{\varepsilon, \gamma}$ and $P_{b}^{\varepsilon, \gamma}$ acting on $u \in P \mathcal{H}^{s}$.

PROPOSITION 4.11 (Adjoints). - Let $a^{*}$ denote the adjoint of the matrix symbol $a \in \Gamma_{1}^{\mu}$ and let $\left(T_{a}^{\varepsilon, \gamma}\right)^{*}$ be the adjoint operator of $T_{a}^{\varepsilon, \gamma}$. There is a $C$ such that for $\varepsilon \in(0,1], \gamma \geqslant 1$ and $u \in \mathcal{H}^{s}$ :

$$
\left\|\left(T_{a}^{\varepsilon, \gamma}\right)^{*}-T_{a^{*}}^{\varepsilon, \gamma} u\right\|_{s-\mu+1, \varepsilon, \gamma} \leqslant C \varepsilon\|u\|_{s, \varepsilon, \gamma} .
$$

The same inequality is true for adjoints of operators $P_{a}^{\varepsilon, \gamma}$ acting on $u \in P \mathcal{H}^{s}$.

Proposition 4.12 (Commutators). - For $a \in \Gamma_{1}^{\mu}$ and $u \in \mathcal{H}^{s}$ we have

$$
\left[\partial, T_{a}^{\varepsilon, \gamma}\right] u=T_{\partial a}^{\varepsilon, \gamma} u,
$$

for $\partial=\partial_{t}$ or $\partial_{y_{j}}$. A similar result holds in the parabolic calculus.

Proposition 4.13 (Gårding inequalities). - Consider symbols $a \in \Gamma_{1}^{\mu}$ and $w \in \Gamma_{1}^{0}$. Suppose that there is $\chi \in \Gamma_{1}^{0}$ and $c>0$ such that $\chi \geqslant 0, \chi w=w$ and

$$
\chi^{2}(t, y, x, \zeta) \Re a(t, y, x, \zeta) \geqslant c \chi^{2}(t, y, x, \zeta)\langle\zeta\rangle^{\mu} \quad \text { for all }(t, y, x, \zeta) .
$$

Then there is $C$ such that for all $\varepsilon \in(0,1], \gamma \geqslant 1$ and $u \in \mathcal{H}^{\mu / 2}$ :

$$
\frac{c}{2}\left\|T_{w}^{\varepsilon, \gamma} u\right\|_{\mu / 2, \varepsilon, \gamma}^{2} \leqslant \Re\left(T_{a}^{\varepsilon, \gamma} T_{w}^{\varepsilon, \gamma} u, T_{w}^{\varepsilon, \gamma} u\right)_{L^{2}}+C \varepsilon^{2}\|u\|_{\mu / 2-1, \varepsilon, \gamma}^{2} .
$$

The same inequality holds for operators $P_{a}^{\varepsilon, \gamma}$ acting on $u \in P \mathcal{H}^{\mu / 2}$.

\subsubsection{Paraproducts}

Paraproducts are paradifferential operators associated to symbols independent of $\zeta$. The following two propositions are used to estimate the errors introduced in the passage from differential operators to their paradifferential counterparts. They can also be used to estimate errors caused by passage from one calculus to the other.

Definition 4.14. - For $k \in \mathbb{N}$, let $\mathcal{W}^{k}$ denote the space of functions $a(t, y, x)$ on $\mathbb{R}^{d+1}$ such that $\partial_{t, y}^{\beta} a \in L^{\infty}\left(\mathbb{R}^{d+1}\right)$ for $|\beta| \leqslant k$. We use the same notation for functions defined just on $\pm x \geqslant 0$.

Observe that

$$
\mathcal{W}^{k} \subset \Gamma_{k}^{0} \cap P \Gamma_{k}^{0}
$$

$4^{\mathrm{e}}$ SÉRIE - TOME $39-2006-\mathrm{N}^{\circ} 1$ 
PROPOSITION 4.15 (Homogeneous paraproducts). - For any $a \in \mathcal{W}^{1}$ there is a constant $C$ such that for all $\varepsilon \in(0,1], \gamma \geqslant 1$ and $u \in \mathcal{H}^{1}$ :

$$
\begin{gathered}
\left\|a u-T_{a}^{\varepsilon, \gamma} u\right\|_{1, \varepsilon, \gamma} \leqslant C \varepsilon\|u\|_{0, \varepsilon, \gamma}, \\
\gamma\left\|a u-T_{a}^{\varepsilon, \gamma} u\right\|_{0, \varepsilon, \gamma}+\left\|a \partial u-T_{a}^{\varepsilon, \gamma} \partial u\right\|_{0, \varepsilon, \gamma} \leqslant C\|u\|_{0, \varepsilon, \gamma}, \quad \text { for } \partial=\partial_{t} \text { or } \partial_{y_{j}} .
\end{gathered}
$$

Proposition 4.16 (Parabolic paraproducts). - For any $a \in \mathcal{W}^{1}$ there is a constant $C$ such that for all $\varepsilon \in(0,1], \gamma \geqslant 1$ and $u \in P \mathcal{H}^{1}$ :

$$
\begin{gathered}
\left\|a u-P_{a}^{\varepsilon, \gamma} u\right\|_{1, \varepsilon, \gamma} \leqslant C \varepsilon\|u\|_{0, \varepsilon, \gamma}, \\
\left\|a \partial_{y_{j}} u-P_{a}^{\varepsilon, \gamma} \partial_{y_{j}} u\right\|_{0, \varepsilon, \gamma} \leqslant C\|u\|_{0, \varepsilon, \gamma}, \\
\gamma\left\|a u-P_{a}^{\varepsilon, \gamma} u\right\|_{0, \varepsilon, \gamma}+\left\|a \partial_{t} u-P_{a}^{\varepsilon, \gamma} \partial_{t} u\right\|_{0, \varepsilon, \gamma}+\sum_{|\alpha|=2} \varepsilon\left\|a \partial_{y}^{\alpha} u-P_{a}^{\varepsilon, \gamma} \partial_{y}^{\alpha} u\right\|_{0, \varepsilon, \gamma} \\
\leqslant C\|u\|_{1, \varepsilon, \gamma} .
\end{gathered}
$$

Remark 4.17. - The difference between the above two propositions is due to the fact that the symbol $i \tau+\gamma$ is of order two in the parabolic calculus, but of order one in the homogeneous calculus.

\subsection{High frequency estimate}

\subsubsection{Paralinearization}

The first step is to replace the problem (4.34) by its counterpart in terms of homogeneous paradifferential operators. We will continue to write $g^{i j}$ for the entries of $G$, suppressing the $\varepsilon$-dependence. The regularity hypothesis, Assumption 4.1, and Remark 4.7 imply

LEMmA 4.18. - The families of symbols $g^{11}, g^{12}, g^{31}, g^{33}$, and $n_{2}^{\varepsilon}$ are bounded in $\Gamma_{2}^{1}$. The families $g^{13}$ and $n_{1}^{\varepsilon}$ are bounded in $\Gamma_{2}^{0}$, while $g^{32}$ is bounded in $\Gamma_{2}^{2}$.

Thus, Proposition 4.15 gives (suppressing superscripts $\varepsilon, \gamma$ on $T$ )

$$
\begin{aligned}
& \frac{1}{\varepsilon}\left\|\left(g^{i j}-T_{g^{i j}}\right) u_{j}\right\| \leqslant C\left\|u_{j}\right\|, \quad \text { except for the case } g^{32}, \\
& \frac{1}{\varepsilon}\left|\left(n_{j}^{\varepsilon}-T_{n_{j}^{\varepsilon}}\right) \nabla_{\gamma} \psi\right| \leqslant C|\psi|_{|\zeta|}, \quad \text { for } j=1,2 .
\end{aligned}
$$

To handle $g^{32}$ we write

$$
g^{32}(t, y, x, \zeta)=g_{a}^{32}(t, y, x, \zeta)+\sum_{j, k=1}^{d-1} a_{i j}(t, y, x) \eta_{i} \eta_{j},
$$

where $g_{a}^{32}$ is bounded in $\Gamma_{1}^{1}$ and the $a_{i j}$ are bounded in $\mathcal{W}^{2}$ (4.14). For the $g_{a}^{32}$ we have an estimate like (4.51). To estimate the second term on the right in (4.52), we use the next lemma.

Lemma 4.19. - Let $a(t, y, x) \in \mathcal{W}^{2}$. Then for $u \in \mathcal{H}^{2}$ we have

$$
\varepsilon\left\|\left(a-T_{a}\right) \partial_{y_{j}} \partial_{y_{k}} u\right\|_{\Lambda_{\varepsilon}^{-1}} \leqslant C\|u\| .
$$

Proof. - Let $\Lambda_{\varepsilon}(D)$ denote operator given by the Fourier multiplier $\Lambda_{\varepsilon}(\zeta)$. From Proposition 4.12 we have

$$
\begin{aligned}
& \varepsilon\left\|\left(a-T_{a}\right) \partial_{y_{j}} \partial_{y_{k}} u\right\|_{\Lambda_{\varepsilon}^{-1}} \\
& \quad \leqslant \varepsilon\left\|\Lambda_{\varepsilon}^{-1}(D) \partial_{j}\left(a-T_{a}\right) \partial_{k} u\right\|+\varepsilon\left\|\Lambda_{\varepsilon}^{-1}(D)\left(\partial_{j} a-T_{\partial_{j} a}\right) \partial_{k} u\right\| .
\end{aligned}
$$


The second term is $\leqslant C \varepsilon\|u\|$ by Proposition 4.15. Since $\left|\varepsilon \eta_{j}\right| \Lambda_{\varepsilon}^{-1}(\zeta) \leqslant 1$, Proposition 4.15 implies the first term is $\leqslant C\|u\|$.

Remark 4.20. - The lemma implies

$$
\frac{1}{\varepsilon}\left\|\left(g^{32}-T_{g^{32}}\right) u_{2}\right\|_{\Lambda_{\varepsilon}^{-1}} \leqslant C\left\|u_{2}\right\| .
$$

In addition $\ell^{\varepsilon}:=\ell\left(q^{\varepsilon}(t, y, 0)\right)$ is bounded in $\mathcal{W}^{1}$, so by Proposition 4.16

$$
\left|\ell^{\varepsilon} \cdot u_{2}-P_{\ell^{\varepsilon}} u_{2}\right|_{\Lambda_{\varepsilon}} \leqslant C \varepsilon\left|u_{2}\right| \text {. }
$$

From (4.55), (4.56), and (4.51) we conclude that if $(U, \psi)$ satisfies the problem (4.34), then it satisfies

$$
\begin{gathered}
\partial_{x} U-\frac{1}{\varepsilon} T_{g^{\varepsilon}} U=\left(\begin{array}{c}
h_{1}^{\prime} \\
0 \\
h_{2}^{\prime}
\end{array}\right)+\frac{1}{\varepsilon}\left(\begin{array}{c}
T_{n_{1}^{\varepsilon}} \nabla_{\gamma} \psi \\
0 \\
T_{n_{2}^{\varepsilon}} \nabla_{\gamma} \psi
\end{array}\right), \\
{[U]=0, \quad\left(\partial_{t}+\gamma\right) \psi-\varepsilon \triangle_{y} \psi+P_{\ell^{\varepsilon}} u_{2}=e \quad \text { on } x=0,}
\end{gathered}
$$

where

$$
\begin{aligned}
\left\|h_{1}^{\prime}\right\|+\left\|h_{2}^{\prime}\right\|_{\Lambda_{\varepsilon}^{-1}} & \leqslant C\left(\left\|h_{1}\right\|+\left\|h_{2}\right\|_{\Lambda_{\varepsilon}^{-1}}+\left\|\left(u_{1}, u_{2}, u_{3}\right)\right\|+|\psi|_{|\zeta|}\right), \\
|e|_{\Lambda_{\varepsilon}} & \leqslant C \varepsilon\left|u_{2}\right| .
\end{aligned}
$$

In particular it suffices to prove the high frequency estimates (4.21), (4.22) for $(U, \psi)$ satisfying (4.57) with $\left(h_{1}^{\prime}, h_{2}^{\prime}\right)$ in place of $\left(f_{1}, f_{2}\right)$.

For cutoffs $\beta=\beta_{1}$ or $\beta_{2}$ and $\chi_{H}$ as in (4.21), (4.22), to find the problem satisfied by $\left(T_{\beta \chi_{H}} U, T_{\beta \chi_{H}} \psi\right)$ we apply $T_{\beta \chi_{H}}$ to (4.57) and use Proposition 4.10 to estimate commutator errors. We find

$$
\begin{gathered}
\partial_{x} T_{\beta \chi_{H}} U-\frac{1}{\varepsilon} T_{g^{\varepsilon}} T_{\beta \chi_{H}} U=\left(\begin{array}{c}
h_{1}^{\prime} \\
0 \\
h_{2}^{\prime}
\end{array}\right)+\frac{1}{\varepsilon}\left(\begin{array}{c}
T_{n_{1}^{\varepsilon}} \nabla_{\gamma} T_{\beta \chi_{H}} \psi \\
0 \\
T_{n_{2}^{\varepsilon}} \nabla_{\gamma} T_{\beta \chi_{H}} \psi
\end{array}\right), \\
{\left[T_{\beta \chi_{H}} U\right]=0, \quad\left(\partial_{t}+\gamma-\varepsilon \triangle_{y}\right) P_{\beta \chi_{H}} \psi+P_{\ell^{\varepsilon}} P_{\beta \chi_{H}} u_{2}=e \quad \text { on } x=0,}
\end{gathered}
$$

for a new $\left(h_{1}^{\prime}, h_{2}^{\prime}\right)$ and $e$ satisfying the estimates (4.58); note $T_{\beta \chi_{H}}=P_{\beta \chi_{H}}$ since the symbol depends on $\zeta$ alone. The commutator involving $T_{g^{32}}$ again requires special treatment; it is handled by an argument similar to the proof of Lemma 4.19.

\subsubsection{Reduction to the partially linearized case}

We begin by rewriting the identity (3.137) in first-order form:

$$
\left(\partial_{z}-G\right)\left(\begin{array}{c}
W_{z} \\
\partial_{z}^{2} W_{2}
\end{array}\right)=\mathcal{J}\left(\mathcal{K}-\partial_{z} \mathcal{P}+\left(\begin{array}{c}
0 \\
r \partial_{z}^{2} W_{1}
\end{array}\right)\right),
$$

for $\mathcal{J}(z, q)$ as in (3.33). Setting

$$
\left(\begin{array}{c}
\mathcal{N}_{1}(z, q, \zeta) \\
0 \\
\mathcal{N}_{2}(z, q, \zeta)
\end{array}\right)=\mathcal{J} \mathcal{K}, \quad \mathcal{N}_{i}^{\varepsilon}:=\kappa(x) \mathcal{N}_{i}\left(\frac{x}{\varepsilon}, q^{\varepsilon}, \zeta\right), \quad \text { and } \quad \theta:=\frac{1}{\varepsilon} T_{\beta \chi_{H}} \psi,
$$

we may rewrite the front term in (4.59)

$$
\frac{1}{\varepsilon}\left(\begin{array}{c}
T_{n_{1}^{\varepsilon}} \nabla_{\gamma} T_{\beta \chi_{H}} \psi \\
0 \\
T_{n_{2}^{\varepsilon}} \nabla_{\gamma} T_{\beta \chi_{H}} \psi
\end{array}\right)=\frac{1}{\varepsilon}\left(\begin{array}{c}
T_{\mathcal{N}_{1}^{\varepsilon}} \theta \\
0 \\
T_{\mathcal{N}_{2}^{\varepsilon}} \theta
\end{array}\right)
$$

$4^{\text {e }}$ SÉRIE - TOME $39-2006-\mathrm{N}^{\circ} 1$ 
Introduce symbols

$$
\begin{aligned}
\left(\begin{array}{c}
e_{1}(z, q, \zeta) \\
0 \\
e_{2}(z, q, \zeta)
\end{array}\right) & =\mathcal{J}\left(-\partial_{z} \mathcal{P}+\left(\begin{array}{c}
0 \\
r \partial_{z}^{2} W_{1}
\end{array}\right)\right) \\
e_{i}^{\varepsilon} & =\varepsilon^{-1} \kappa(x) e_{i}\left(\frac{x}{\varepsilon}, q^{\varepsilon}\right) \\
s^{\varepsilon} & =\left(s_{1}^{\varepsilon}, s_{2}^{\varepsilon}\right):=\kappa(x) \partial_{z} W\left(\frac{x}{\varepsilon}, q^{\varepsilon}\right)
\end{aligned}
$$

LEMMA 4.21. -

(a) The families $e_{1}^{\varepsilon}, e_{2}^{\varepsilon}, s^{\varepsilon}$, and $\varepsilon \partial_{x} s_{2}^{\varepsilon}$ are bounded in $\Gamma_{1}^{0} ; \mathcal{N}_{1}^{\varepsilon}$ is bounded in $\Gamma_{1}^{1}$ and $\mathcal{N}_{2}^{\varepsilon}$ is bounded in $\Gamma_{1}^{2}$.

(b) The families

$$
\begin{aligned}
& \tilde{e}_{1}^{\varepsilon}:=\partial_{x} s_{1}^{\varepsilon}-\frac{1}{\varepsilon} g^{11} s_{1}^{\varepsilon}-\frac{1}{\varepsilon} g^{12} s_{2}^{\varepsilon}-g^{13} \partial_{x} s_{2}^{\varepsilon}-\frac{1}{\varepsilon} \mathcal{N}_{1}^{\varepsilon}, \\
& \tilde{e}_{2}^{\varepsilon}:=\varepsilon \partial_{x}^{2} s_{2}^{\varepsilon}-\frac{1}{\varepsilon} g^{31} s_{1}^{\varepsilon}-\frac{1}{\varepsilon} g^{32} s_{2}^{\varepsilon}-g^{33} \partial_{x} s_{2}^{\varepsilon}-\frac{1}{\varepsilon} \mathcal{N}_{2}^{\varepsilon}
\end{aligned}
$$

are bounded in $\Gamma_{1}^{0}$ and $\Gamma_{1}^{1}$, respectively. Moreover, $s^{\varepsilon}$ is $C^{\infty}$ in $x$ across $x=0$.

Proof. - The statement for $s^{\varepsilon}$ and $\varepsilon \partial_{x} s^{\varepsilon}$ is clear from the definition of $q^{\varepsilon}$ (4.3) and the regularity assumption (H10); for $\mathcal{N}_{i}^{\varepsilon}$ recall Remark 4.7. To see the boundedness of the $e_{i}^{\varepsilon}$, recall that $W(z, q)=W_{0}(z, p)+u^{\prime}$, so when $u^{\prime}=0$ and $h^{\prime}=0, W$ is an exact solution of the profile equation $\mathcal{P}(z, q)=0$. Thus

$$
\mathcal{J} \partial_{z} \mathcal{P}(z, q)=u^{\prime} W_{a}(z, q)+h^{\prime} W_{b}(z, q),
$$

where $W_{a}, W_{b}$ are smooth with exponential decay in $z$. The $h_{\varepsilon}^{\prime} W_{b}$ contribution to $e_{i}^{\varepsilon}$ is bounded by part (d) of (H10). We have

$$
u_{\varepsilon}^{\prime}(t, y, x)=\left.u_{\varepsilon}^{\prime}\right|_{x=0}+x v_{\varepsilon}(t, y, x)
$$

so boundedness of the $u_{\varepsilon}^{\prime} W_{a}$ contribution follows from parts (b) and (c) of (H10) and the boundedness of $\frac{x}{\varepsilon} W_{a}\left(\frac{x}{\varepsilon}, q^{\varepsilon}\right)$. The $r_{\varepsilon} \partial_{z}^{2} W_{1}$ term is bounded by part (e) of (H10).

The identity (4.60) implies

$$
\tilde{e}_{1}^{\varepsilon}=e_{1}^{\varepsilon}+\left(\partial_{x} \kappa \partial_{z} W_{1}+\kappa \partial_{z q}^{2} W_{1} \partial_{x} q^{\varepsilon}+g^{13} \partial_{x} \kappa \partial_{z} W_{2}+g^{13} \kappa \partial_{z q}^{2} W_{2} \partial_{x} q^{\varepsilon}\right),
$$

where the sum in parentheses is bounded in $\Gamma_{1}^{0}$ by the regularity assumption (H10) and the fact that $g^{13}$ is of order zero. The term $\tilde{e}_{2}^{\varepsilon}$ is treated similarly, but recall that $g^{33}$ is of order one.

The regularity of $s^{\varepsilon}$ in $x$ follows from $\partial_{z} W(z, q)=\partial_{z} W_{0}(z, p)$.

Next we make a change of unknown corresponding to $u^{\#}=u-\psi \partial_{z} W$ (3.10) in the frozen coefficient argument. Again with $\beta=\beta_{1}$ or $\beta_{2}$ and $\theta=\frac{1}{\varepsilon} T_{\beta \chi_{H}} \psi$ set

$$
V=\left(\begin{array}{c}
v_{1} \\
v_{2} \\
v_{3}
\end{array}\right):=T_{\beta \chi_{H}} U-T_{S^{\varepsilon}} \theta, \quad S^{\varepsilon}=\left(\begin{array}{c}
s^{\varepsilon} \\
\varepsilon \partial_{x} s_{2}^{\varepsilon}
\end{array}\right),
$$

and introduce the bounded family in $P \Gamma_{1}^{2}$

$$
c^{\varepsilon}(t, y, \zeta)=i \tau+\gamma+|\eta|^{2}+\ell^{\varepsilon} \cdot \partial_{z} W_{2}\left(0, q^{\varepsilon}(t, y, 0)\right) .
$$


PROPOSITION 4.22. - $V$ and $\theta$ satisfy

$$
\begin{aligned}
& \text { (a) } \partial_{x} V-\frac{1}{\varepsilon} T_{g^{\varepsilon}} V=\left(\begin{array}{c}
h_{1}^{\prime \prime} \\
0 \\
h_{2}^{\prime \prime}
\end{array}\right), \quad[V]=0, \\
& \text { (b) } P_{c^{\varepsilon}} \theta+P_{\ell^{\varepsilon}} v_{2}(0)=e^{\prime},
\end{aligned}
$$

where (in place of (4.58))

$$
\begin{gathered}
\left\|h_{1}^{\prime \prime}\right\|+\left\|h_{2}^{\prime \prime}\right\|_{\Lambda_{\varepsilon}^{-1}} \leqslant C\left(\left\|h_{1}\right\|+\left\|h_{2}\right\|_{\Lambda_{\varepsilon}^{-1}}+\left\|\left(u_{1}, u_{2}, u_{3}\right)\right\|+\left|T_{\beta \chi_{H}} \psi\right|_{|\zeta|}\right), \\
\left|e^{\prime}\right|_{\Lambda_{\varepsilon}} \leqslant C \varepsilon\left|u_{2}(0)\right|+\left|T_{\beta \chi_{H}} \psi\right| .
\end{gathered}
$$

Proof. - Using (4.59) we compute

$$
\begin{aligned}
\partial_{x} V-\frac{1}{\varepsilon} T_{g^{\varepsilon}} V & =\left(\begin{array}{c}
h_{1}^{\prime} \\
0 \\
h_{2}^{\prime}
\end{array}\right)+\frac{1}{\varepsilon}\left(\begin{array}{c}
T_{\mathcal{N}_{1}^{\varepsilon}} \theta \\
0 \\
T_{\mathcal{N}_{2}^{\varepsilon}} \theta
\end{array}\right)-\left(\partial_{x}-\frac{1}{\varepsilon} T_{g^{\varepsilon}}\right) T_{S^{\varepsilon}} \theta \\
& =\left(\begin{array}{c}
h_{1}^{\prime} \\
0 \\
h_{2}^{\prime}
\end{array}\right)+\left(\begin{array}{c}
T_{\tilde{e}_{1}^{\varepsilon}} \\
0 \\
T_{\tilde{e}_{2}^{\varepsilon}}
\end{array}\right) \theta+\left(\begin{array}{c}
E_{1} \\
0 \\
E_{2}
\end{array}\right) \theta:=\left(\begin{array}{c}
h_{1}^{\prime \prime} \\
0 \\
h_{2}^{\prime \prime}
\end{array}\right),
\end{aligned}
$$

where by Lemma 4.21

$$
\begin{aligned}
\left\|T_{\tilde{e}_{1}^{\varepsilon}} \theta\right\| & \leqslant C|\theta| \leqslant C\left|T_{\beta \chi_{H}} \psi\right| / \varepsilon \leqslant C\left|T_{\beta \chi_{H}} \psi\right|_{|\zeta|}, \\
\left\|T_{\tilde{e}_{2}^{\varepsilon}} \theta\right\|_{\Lambda_{\varepsilon}^{-1}} & \leqslant C\left\|T_{\tilde{e}_{2}^{\varepsilon}} \theta\right\| \leqslant C|\theta|_{\langle\varepsilon \zeta\rangle} \leqslant C\left|T_{\beta \chi_{H}} \psi\right|_{|\zeta|},
\end{aligned}
$$

and $\left\|E_{1} \theta\right\|+\left\|E_{2} \theta\right\|_{\Lambda_{\varepsilon}^{-1}} \leqslant C|\theta| . E_{1}$ and $E_{2}$ are sums of composition errors like

$$
\frac{1}{\varepsilon}\left(T_{g^{11}} T_{s_{1}^{\varepsilon}}-T_{g^{11} s_{1}^{\varepsilon}}\right) \quad \text { and } \quad \frac{1}{\varepsilon}\left(T_{g^{32}} T_{s_{2}^{\varepsilon}}-T_{g^{32} s_{2}^{\varepsilon}}\right)
$$

respectively. The second term in (4.74) is again handled by an argument like the proof of Lemma 4.19.

The smoothness of $s^{\varepsilon}$ in $x$ and $\left[T_{\beta \chi_{H}} U\right]=0$ implies $[V]=0$.

Finally, since $P_{\beta \chi_{H}}=T_{\beta \chi_{H}}$, we may write

$$
\begin{aligned}
P_{c^{\varepsilon}} \theta+P_{\ell^{\varepsilon}} v_{2} & =\left(P_{i \tau+\gamma+|\eta|^{2}} \theta+P_{\ell^{\varepsilon}} P_{\beta \chi_{H}} u_{2}\right)+\left(P_{\ell^{\varepsilon} s_{2}^{\varepsilon}}-P_{\ell^{\varepsilon}} P_{s_{2}^{\varepsilon}}\right) \theta+P_{\ell^{\varepsilon}}\left(P_{s_{2}^{\varepsilon}}-T_{s_{2}^{\varepsilon}}\right) \theta \\
& =e+E^{\prime}+E^{\prime \prime},
\end{aligned}
$$

for $e$ as in (4.59) and

$$
\begin{aligned}
\left|E^{\prime}\right|_{\Lambda_{\varepsilon}} \leqslant C \varepsilon|\theta| & \text { (by Proposition 4.10), } \\
\left|E^{\prime \prime}\right|_{\Lambda_{\varepsilon}} \leqslant C \varepsilon|\theta| & \text { (by Propositions 4.15 and 4.16). }
\end{aligned}
$$

The next proposition, an estimate for the partially linearized problem (4.70)(a), is the main step in the proof of the high frequency estimate of Theorem 4.4. The proof is given in the next section.

PROPOSITION 4.23. - Under the assumptions of Theorem 4.4, consider $V=\left(v_{1}, v_{2}, v_{3}\right)$ as defined in (4.68) and satisfying (4.70)(a). There exists $\varepsilon_{0}$ such that for $\gamma \geqslant 1$ and $0<\varepsilon \leqslant \varepsilon_{0}$ the following estimates hold. When $\beta=\beta_{1}$,

$4^{\text {e }}$ SÉRIE - TOME $39-2006-\mathrm{N}^{\circ} 1$ 


$$
\begin{aligned}
& \left(\frac{1}{\varepsilon}+\gamma\right)\left\|v_{1}\right\|+\frac{1}{\varepsilon}\left\|v_{2}\right\| \Lambda_{\varepsilon}+\frac{1}{\varepsilon}\left\|v_{3}\right\|+\left(\frac{1}{\varepsilon}+\gamma\right)^{1 / 2}\left|v_{1}(0)\right| \\
& \quad+\frac{1}{\sqrt{\varepsilon}}\left|v_{2}(0)\right|_{\Lambda_{\varepsilon}^{1 / 2}}+\frac{1}{\sqrt{\varepsilon}}\left|v_{3}(0)\right|_{\Lambda_{\varepsilon}^{-1 / 2}} \\
& \leqslant C\left(\left\|h_{1}^{\prime \prime}\right\|+\left\|h_{2}^{\prime \prime}\right\|_{\Lambda_{\varepsilon}^{-1}}+\text { a.t. }\right) .
\end{aligned}
$$

When $\beta=\beta_{2}$,

$$
\begin{aligned}
& \text { (4.78) } \frac{1}{\varepsilon}\left\|v_{1}\right\|_{\langle\varepsilon \zeta\rangle}+\frac{1}{\varepsilon}\left\|v_{2}\right\|_{\Lambda_{\varepsilon}}+\frac{1}{\varepsilon}\left\|v_{3}\right\|+\frac{1}{\sqrt{\varepsilon}}\left|v_{1}(0)\right|_{\langle\varepsilon \zeta\rangle^{1 / 2}}+\frac{1}{\sqrt{\varepsilon}}\left|v_{2}(0)\right|_{\Lambda_{\varepsilon}^{1 / 2}}+\frac{1}{\sqrt{\varepsilon}}\left|v_{3}(0)\right|_{\Lambda_{\varepsilon}^{-1 / 2}} \\
& \leqslant C\left(\left\|h_{1}^{\prime \prime}\right\|+\left\|h_{2}^{\prime \prime}\right\|_{\Lambda_{\varepsilon}^{-1}}+\text { a.t. }\right) .
\end{aligned}
$$

Here, as in Theorem 4.4, "a.t." stands for a finite sum of terms that can be absorbed by the sum of the left sides of (4.19)-(4.22) by taking $\gamma$ large and $\varepsilon$ small.

Next we show how to use the extra boundary condition (4.70)(b) to estimate $\theta$ in terms of the trace of $v_{2}$.

Proposition 4.24. - Under the assumptions of Proposition 4.23, for $\beta=\beta_{1}$ or $\beta_{2}$ and $\varepsilon$ small enough there holds

$$
\frac{1}{\varepsilon^{3 / 2}}\left|T_{\beta \chi_{H}} \psi\right|_{\Lambda_{\varepsilon}^{5 / 2}}=\frac{1}{\varepsilon^{1 / 2}}|\theta|_{\Lambda_{\varepsilon}^{5 / 2}} \leqslant C\left(\frac{1}{\varepsilon^{1 / 2}}\left|v_{2}(0)\right|_{\Lambda_{\varepsilon}^{1 / 2}}+\text { a.t. }\right) .
$$

Proof. - The choice of $\ell(q)$ (2.60) and (H10) imply that for $\varepsilon$ small

$$
\ell\left(q^{\varepsilon}\right) \cdot \partial_{z} W_{2}\left(0, q^{\varepsilon}\right) \gtrsim 1,
$$

and hence

$$
1+|\tau|+\gamma+|\eta|^{2} \lesssim\left|c^{\varepsilon}\right|
$$

Thus, $c^{\varepsilon}$ is elliptic and $1 / c^{\varepsilon} \in P \Gamma_{1}^{-2}$. Applying $P_{1 / c^{\varepsilon}}$ to (4.70)(b) and using the parabolic calculus we obtain

$$
\begin{aligned}
\left|P_{1 / c^{\varepsilon}} P_{c^{\varepsilon}} \theta-\theta\right|_{\Lambda_{\varepsilon}^{5 / 2}} & \lesssim \varepsilon|\theta|_{\Lambda_{\varepsilon}^{3 / 2}}, \\
|\theta|_{\Lambda_{\varepsilon}^{5 / 2}} & \lesssim\left|P_{1 / c^{\varepsilon}} e^{\prime}\right|_{\Lambda_{\varepsilon}^{5 / 2}}+\left|P_{1 / c^{\varepsilon}} P_{\ell^{\varepsilon}} v_{2}(0)\right|_{\Lambda_{\varepsilon}^{5 / 2}}+\varepsilon|\theta|_{\Lambda_{\varepsilon}^{3 / 2}} .
\end{aligned}
$$

Thus,

$$
\frac{1}{\varepsilon^{1 / 2}}|\theta|_{\Lambda_{\varepsilon}^{5 / 2}} \lesssim \frac{1}{\varepsilon^{1 / 2}}\left|v_{2}(0)\right|_{\Lambda_{\varepsilon}^{1 / 2}}+\frac{1}{\varepsilon^{1 / 2}}\left|T_{\beta \chi_{H}} \psi\right|_{\Lambda_{\varepsilon}^{3 / 2}}+\frac{1}{\varepsilon^{1 / 2}}\left|e^{\prime}\right|_{\Lambda_{\varepsilon}^{1 / 2}},
$$

from which the result follows by (4.71).

We conclude this section by showing how Propositions 4.23 and 4.24 imply the high frequency estimates of Theorem 4.4.

Proof of estimates (4.21) and (4.22) assuming Proposition 4.23. - The case $\beta=\beta_{2}$. Adding the estimates (4.78) and (4.79) and recalling (4.71), we deduce

$$
\begin{aligned}
& \frac{1}{\varepsilon}\left\|v_{1}\right\|_{\langle\varepsilon \zeta\rangle}+\frac{1}{\varepsilon}\left\|v_{2}\right\|_{\Lambda_{\varepsilon}}+\frac{1}{\varepsilon}\left\|v_{3}\right\|+\frac{1}{\sqrt{\varepsilon}}\left|v_{1}(0)\right|_{\langle\varepsilon \zeta\rangle^{1 / 2}} \\
& \quad+\frac{1}{\sqrt{\varepsilon}}\left|v_{2}(0)\right|_{\Lambda_{\varepsilon}^{1 / 2}}+\frac{1}{\sqrt{\varepsilon}}\left|v_{3}(0)\right|_{\Lambda_{\varepsilon}^{-1 / 2}}+\frac{1}{\varepsilon^{3 / 2}}\left|T_{\beta \chi_{H}} \psi\right|_{\Lambda_{\varepsilon}^{5 / 2}} \\
& \leqslant C\left(\left\|f_{1}\right\|+\left\|f_{2}\right\|_{\Lambda_{\varepsilon}^{-1}}+\text { a.t. }\right) .
\end{aligned}
$$

ANNALES SCIENTIFIQUES DE L'ÉCOLE NORMALE SUPÉRIEURE 
Note first that

$$
\frac{\Lambda_{\varepsilon}}{\varepsilon} \lesssim \frac{\langle\varepsilon \zeta\rangle}{\varepsilon} \lesssim \frac{\Lambda_{\varepsilon}^{2}}{\varepsilon}
$$

Write $T_{\beta_{2} \chi_{H}} U=V+T_{S^{\varepsilon}} \theta$ and use the exponential decay of $S^{\varepsilon}$ in $\frac{x}{\varepsilon}$ to see that for each $x$

$$
\frac{1}{\varepsilon}\left|T_{S^{\varepsilon}} \theta(x)\right|_{\langle\varepsilon \zeta\rangle} \lesssim \frac{1}{\varepsilon} e^{-\delta|x| / \varepsilon}|\theta|_{\langle\varepsilon \zeta\rangle} .
$$

Taking the $L^{2}$ norm in $x$ gives

$$
\frac{1}{\varepsilon}\left\|T_{S^{\varepsilon}} \theta\right\|_{\langle\varepsilon \zeta\rangle} \lesssim \frac{1}{\sqrt{\varepsilon}}|\theta|_{\langle\varepsilon \zeta\rangle} \lesssim \frac{1}{\varepsilon^{3 / 2}}\left|T_{\beta \chi_{H}} \psi\right|_{\langle\varepsilon \zeta\rangle} \lesssim \frac{1}{\varepsilon^{3 / 2}}\left|T_{\beta \chi_{H}} \psi\right|_{\Lambda_{\varepsilon}^{2}} .
$$

For the trace at $x=0$ we have

$$
\frac{1}{\sqrt{\varepsilon}}\left|T_{S^{\varepsilon}} \theta\right|_{\langle\varepsilon \zeta\rangle^{1 / 2}} \lesssim \frac{1}{\sqrt{\varepsilon}}|\theta|_{\langle\varepsilon \zeta\rangle^{1 / 2}}=\frac{1}{\varepsilon^{3 / 2}}\left|T_{\beta \chi_{H}} \psi\right|_{\langle\varepsilon \zeta\rangle^{1 / 2}} \lesssim \frac{1}{\varepsilon^{3 / 2}}\left|T_{\beta \chi_{H}} \psi\right|_{\Lambda_{\varepsilon}} .
$$

Together with (4.83) and (4.82), the last two estimates imply estimate (4.22) of Theorem 4.4.

The case $\beta=\beta_{1}$. We have $\frac{1}{\varepsilon}\langle\varepsilon \zeta\rangle \gtrsim \gamma+\frac{1}{\varepsilon}$, so the above estimates imply (4.21) as well.

\subsubsection{Estimate for the partially linearized problem}

In this section we complete the proof of the high frequency estimates by proving Proposition 4.23. We will do this by quantizing the arguments of Section 3.2.7; in particular, we must show that the errors introduced by use of the paradifferential calculus are always absorbable. We begin with the more difficult case.

1. The case $\beta=\beta_{1}$ : decoupling. We use the conjugator $\mathcal{W}(z, q, \zeta)$ (3.47), which is initially defined only for $|\zeta| \geqslant R, 0 \leqslant \gamma \leqslant \delta|\zeta|$ and $q=\left(p, u^{\prime}, h^{\prime}, r\right)$ with $\left|u^{\prime}, h^{\prime}, r\right|$ small. Extend $W_{a}^{21}$ and $W_{b}^{21}$ to all $(q, \zeta)$ as elements of $\Gamma^{0}$ which vanish for $|\zeta| \leqslant 1$ (here $R \gg 1$ ). Then

$$
\mathcal{W}^{\varepsilon}(t, y, x, \zeta):=\mathcal{W}\left(\frac{x}{\varepsilon}, q^{\varepsilon}, \zeta\right) \in \Gamma_{1}^{0} .
$$

We will often drop the superscript $\varepsilon$ in this section and, for example, write simply $W_{a}^{21}|\zeta|^{-1}$ and $W_{b}^{21}$ for the $(2,1)$ and $(3,1)$ components of $\mathcal{W}^{\varepsilon}$. We have then

$$
W_{a}^{21}|\zeta|^{-1} \in \Gamma_{1}^{-1}, \quad W_{b}^{21} \in \Gamma_{1}^{0} .
$$

The special structure of $\mathcal{W}$ immediately implies the following convenient identities:

$$
T_{\mathcal{W}} T_{\mathcal{W}^{-1}}=T_{\mathcal{W}^{-1}} T_{\mathcal{W}}=I
$$

there is no composition error in this case.

Remark 4.25. - The regularity assumption (H10) implies that $\left|h_{\varepsilon}^{\prime}, r_{\varepsilon}\right|$ is small for $\varepsilon$ small, while $\left|u_{\varepsilon}^{\prime}\right|$ is small for $\varepsilon$ and $|x|$ small. Recall that in Theorem 4.4 we assume $u^{ \pm}$and $f^{ \pm}$are supported near $x=0$.

It will be useful to have a notation for error operators.

NotATION 4.26. - An operator $r_{\mu}^{\varepsilon, \gamma}$ on the homogeneous scale of Sobolev spaces $\mathcal{H}^{s}$ is said to be of order $\mu$ if for any $s \in \mathbb{R}$ there is a $C$ such that for $\gamma \geqslant 1$ and $\varepsilon \in(0,1]$ the following estimate holds for $u \in \mathcal{H}^{s}$ :

$$
\left\|r_{\mu}^{\varepsilon, \gamma} u\right\|_{s-\mu, \varepsilon, \gamma} \leqslant C\|u\|_{s, \varepsilon, \gamma} .
$$

The same notation is used for operators of order $\mu$ on functions $u(t, y) \in H^{s}$.

$4^{e}$ SÉRIE - TOME $39-2006-\mathrm{N}^{\circ} 1$ 
We will usually drop the superscripts $\varepsilon, \gamma$, and the identity of operators denoted $r_{\mu}$ may change from term to term, line to line, etc. We will use the same notation (when the context is clear) for analogously defined operators of order $\mu$ on the parabolic spaces $P \mathcal{H}^{s}$.

For $V$ as in (4.70) we define $\mathbb{V}=\left(\mathrm{v}_{1}, \mathrm{v}_{2}, \mathrm{\vee}_{3}\right)$ by $V=T_{\mathcal{W}} \mathbb{V}$ and with (4.89) obtain, parallel to $(3.93)$,

$$
\partial_{x} \mathbb{V}-\frac{1}{\varepsilon} T_{\mathcal{W}^{-1}} T_{g} T_{\mathcal{W}} \mathbb{V}=T_{\mathcal{W}^{-1}}\left(\begin{array}{c}
h_{1}^{\prime \prime} \\
0 \\
h_{2}^{\prime \prime}
\end{array}\right)-T_{\mathcal{W}^{-1}} T_{\partial_{x} \mathcal{W}} \mathbb{V}
$$

Setting $g_{0}^{\varepsilon}=G_{0}\left(\frac{x}{\varepsilon}, q^{\varepsilon}, \zeta\right)$ for $G_{0}$ as in (3.48), we have

$$
\begin{aligned}
& \frac{1}{\varepsilon} T_{\mathcal{W}^{-1}} T_{g} T_{\mathcal{W}} \\
& \quad=\frac{1}{\varepsilon} T_{g_{0}}+\frac{1}{\varepsilon}\left(\begin{array}{ccc}
0 & 0 & 0 \\
T_{W_{b}^{21}-W_{a}^{21}|\zeta|^{-1} g^{11}} & 0 & 0 \\
T_{g^{31}+g^{32} W_{a}^{21}|\zeta|^{-1}+g^{33} W_{b}^{21}-W_{b}^{21} g^{11}} & 0 & 0
\end{array}\right)+\left(\begin{array}{ccc}
r_{-1} & 0 & 0 \\
r_{-1} & r_{-1} & r_{-2} \\
r_{0} & r_{0} & r_{-1}
\end{array}\right) \\
& \quad=\frac{1}{\varepsilon} T_{g_{0}}+E_{1}+E_{2} .
\end{aligned}
$$

Remark 4.27. - (1) Note that errors of size $\frac{|\psi|}{\varepsilon}$ are too large to be "a.t." terms because of the contribution from the low frequency regime. We avoid such errors below by setting $\chi_{m h}:=\chi_{M}+\chi_{H}$ and observing

$$
T_{\beta_{1} \chi_{H}} \frac{\psi}{\varepsilon}=T_{\beta_{1} \chi_{H}} T_{\chi_{m h}} \frac{\psi}{\varepsilon} .
$$

With $\psi_{m h}:=T_{\chi_{m h}} \psi$, we have $\frac{\left|\psi_{m h}\right|}{\varepsilon}$ is an a.t. term.

(2) Tracing back the definition of $v_{1}$, we can now write

$$
\mathrm{v}_{1}=v_{1}=T_{\beta_{1} \chi_{H}} u_{1}-T_{s_{1}^{\varepsilon} \beta_{1} \chi_{H}} \frac{\psi_{m h}}{\varepsilon} .
$$

By (3.42) and Remark 4.25, the symbols defining the entries of the first error matrix $E_{1}$ vanish for $\varepsilon+|x|$ small and $\zeta \in \operatorname{supp}\left(\beta_{1} \chi_{H}\right)$. The homogeneous calculus therefore gives for $\varepsilon$ small

$$
E_{1}^{21} \mathrm{v}_{1}=r_{-1} u_{1}+r_{-1} \frac{\psi_{m h}}{\varepsilon} ; \quad E_{1}^{31} \mathrm{v}_{1}=r_{0} u_{1}+r_{0} \frac{\psi_{m h}}{\varepsilon},
$$

where the error operators in the terms involving $\psi$ have compact support in the parameter $x$ by (4.63). The second error matrix $E_{2}$ contains all the composition errors. For example,

$$
\begin{aligned}
\varepsilon E_{2}^{21}= & -T_{W_{a}^{21}|\zeta|^{-1}} T_{g^{11}}-T_{W_{a}^{21}|\zeta|^{-1}} T_{g^{12}} T_{W_{a}^{21}|\zeta|^{-1}}-T_{W_{a}^{21}|\zeta|^{-1}} T_{g^{13}} T_{W_{b}^{21}} \\
& -T_{\left(-W_{a}^{21}|\zeta|^{-1} g^{11}-W_{a}^{21}|\zeta|^{-1} g^{12} W_{a}^{21}|\zeta|^{-1}-W_{a}^{21}|\zeta|^{-1} g^{13} W_{b}^{21}\right)}=\varepsilon r_{-1} .
\end{aligned}
$$

We have $T_{\mathcal{W}^{-1}} T_{\partial_{x} \mathcal{W}}=T_{\mathcal{W}^{-1}} \partial_{x} \mathcal{W}$ with again no composition error, so a short computation shows the right side of (4.91) is

$$
\left(\begin{array}{c}
h_{1}^{\prime \prime} \\
r_{-1} h_{1}^{\prime \prime} \\
r_{0} h_{1}^{\prime \prime}+h_{2}^{\prime \prime}
\end{array}\right)+\frac{1}{\varepsilon}\left(\begin{array}{c}
0 \\
r_{-1} \mathrm{v}_{1} \\
r_{0} \mathrm{v}_{1}
\end{array}\right) .
$$

Putting all this together we may rewrite (4.91) parallel to (3.93) as: 


$$
\begin{aligned}
\partial_{x} \mathbb{V}-\frac{1}{\varepsilon} T_{g_{0}} \mathbb{V}= & E_{1} \mathbb{V}+E_{2} \mathbb{V}+T_{\mathcal{W}-1}\left(\begin{array}{c}
h_{1}^{\prime \prime} \\
0 \\
h_{2}^{\prime \prime}
\end{array}\right)-T_{\mathcal{W}-1} T_{\partial_{x}} \mathcal{W} \mathbb{V} \\
= & \left(\begin{array}{c}
0 \\
r_{-1} u_{1}+r_{-1} \frac{\psi_{m h}}{\varepsilon} \\
r_{0} u_{1}+r_{0} \frac{\psi_{m h}}{\varepsilon}
\end{array}\right)+\left(\begin{array}{c}
r_{-1} \mathrm{v}_{1} \\
r_{-1} \mathrm{v}_{1}+r_{-1} \mathrm{v}_{2}+r_{-2} \mathrm{v}_{3} \\
r_{0} \mathrm{v}_{1}+r_{0} \mathrm{v}_{2}+r_{-1} \mathrm{v}_{3}
\end{array}\right) \\
& +\left(\begin{array}{c}
h_{1}^{\prime \prime} \\
r_{-1} h_{1}^{\prime \prime} \\
r_{0} h_{1}^{\prime \prime}+h_{2}^{\prime \prime}
\end{array}\right)+\frac{1}{\varepsilon}\left(\begin{array}{c}
0 \\
r_{-1} \mathrm{v}_{1} \\
r_{0} \mathrm{v}_{1}
\end{array}\right)
\end{aligned}
$$

Next, for $\widehat{G}^{11}$ as in (3.52) and $M_{i n}^{22}$ as in (3.38), we set

$$
\hat{g}^{11}=\widehat{G}^{11}\left(\frac{x}{\varepsilon}, q^{\varepsilon}, \zeta\right) \text { and } g_{p}=M_{i n}^{22}\left(\frac{x}{\varepsilon}, q^{\varepsilon}, \zeta\right)
$$

and consider the block diagonal symbol

$$
g_{b}=\left(\begin{array}{cc}
\hat{g}^{11} & 0 \\
0 & g_{p}
\end{array}\right) .
$$

Denoting the right side of (4.98) by $\mathcal{F}$ and recalling the form of $G_{0}$ (3.48), we can rewrite (4.98) as a problem in terms of $T_{g_{b}}$ parallel to (3.96):

$$
\begin{gathered}
\partial_{x} \mathbb{V}-\frac{1}{\varepsilon} T_{g_{b}} \mathbb{V}=\mathcal{F}+\frac{1}{\varepsilon}\left(\begin{array}{c}
T_{g^{12} \mathrm{v}_{2}}+r_{0} \mathrm{v}_{3} \\
r_{-1} \mathrm{v}_{1}+r_{0} \mathrm{v}_{2}+r_{-1} \mathrm{v}_{3} \\
r_{0} \mathrm{v}_{1}+T_{-W_{b}^{21}} g^{12} \mathrm{v}_{2}+r_{0} \mathrm{v}_{3}
\end{array}\right) \\
:=\mathcal{H}=\left(\begin{array}{c}
H_{1} \\
H_{2} \\
H_{3}
\end{array}\right), \quad[\mathbb{V}]=0 .
\end{gathered}
$$

As in the frozen coefficient case, it is now easy to check that the desired estimate (4.77) of Proposition 4.23 is implied by the following estimates for $\mathbb{V}=\left(\mathrm{v}_{1}, \mathrm{v}_{2}, \mathrm{v}_{3}\right)$ satisfying (4.101):

$$
\begin{gathered}
\left(\frac{1}{\varepsilon}+\gamma\right)\left\|\mathrm{v}_{1}\right\|+\left(\frac{1}{\varepsilon}+\gamma\right)^{1 / 2}\left|\mathrm{v}_{1}(0)\right| \lesssim\left\|H_{1}\right\|+\text { a.t., } \\
\frac{1}{\varepsilon}\left\|\mathrm{v}_{2}\right\|_{\Lambda_{\varepsilon}}+\frac{1}{\varepsilon}\left\|\mathrm{v}_{3}\right\|+\frac{1}{\sqrt{\varepsilon}}\left|\mathrm{v}_{2}(0)\right|_{\Lambda_{\varepsilon}^{1 / 2}}+\frac{1}{\sqrt{\varepsilon}}\left|\mathrm{v}_{3}(0)\right|_{\Lambda_{\varepsilon}^{-1 / 2}} \lesssim\left\|H_{2}\right\|+\left\|H_{3}\right\|_{\Lambda_{\varepsilon}^{-1}}+\text { a.t., }
\end{gathered}
$$

where "a.t." has the same meaning as in Proposition 4.23. In reducing to (4.102) we have used the special structure of $g^{12}$, which yields (since $|\eta| \Lambda^{-1}(\zeta) \leqslant 1$ )

$$
\begin{aligned}
\frac{1}{\varepsilon}\left\|T_{g^{12}} \mathrm{v}_{2}\right\| & \lesssim \frac{1}{\varepsilon}\left\|\mathrm{v}_{2}\right\|+\left\|\partial_{y} \mathrm{v}_{2}\right\| \lesssim \frac{1}{\varepsilon}\left\|\mathrm{v}_{2}\right\|_{\Lambda_{\varepsilon}}, \\
\frac{1}{\varepsilon}\left\|T_{-W_{b}^{21}} g^{12} \mathrm{v}_{2}\right\|_{\Lambda_{\varepsilon}^{-1}} & \lesssim \frac{1}{\varepsilon}\left\|\mathrm{v}_{2}\right\| .
\end{aligned}
$$

Thus, we have reduced the proof of Proposition 4.23 in the case $\beta=\beta_{1}$ to proving the following proposition.

PROPOSITION 4.28 (The case $\beta=\beta_{1}$ ). - Under the assumptions of Theorem 4.4, consider $\mathbb{V}=T_{\mathcal{W}^{-1}} V=\left(\mathrm{v}_{1}, \mathrm{v}_{2}, \mathrm{v}_{3}\right)$ satisfying (4.101). If $R$ is large enough, there exists $\varepsilon_{0}$ such that for $\gamma \geqslant 1$ and $0<\varepsilon \leqslant \varepsilon_{0}$ the estimates (4.102) hold.

$4^{e}$ SÉRIE - TOME $39-2006-\mathrm{N}^{\circ} 1$ 
2. The hyperbolic block. Consider the problem given by the $(1,1)$ block of $(4.101)$ :

$$
\partial_{x} \mathrm{v}_{1}-\frac{1}{\varepsilon} T_{\hat{g}^{11} \mathrm{v}_{1}}=H_{1}, \quad\left[\mathrm{v}_{1}\right]=0 .
$$

We will perform another conjugation using the matrix $\mathcal{V}(z, q, \zeta)$ as in (3.56):

$$
\mathcal{V}^{-1} \widehat{G}^{11} \mathcal{V}=\Lambda=\left.\operatorname{diag}\left(\mu_{k} I_{s_{k}}+R_{k}\right)\right|_{k=1, \ldots, k_{0}},
$$

where $\mathcal{V}$ is chosen so that (3.59) also holds.

The functions $\mathcal{V}$ and $R_{k}$ are, initially, smoothly defined and satisfy (4.105) for $z \in \mathbb{R}$ and for

$$
\begin{aligned}
& 0 \leqslant \gamma \leqslant \delta|\zeta|,|\zeta| \geqslant R \text {, and } q=\left(p, u^{\prime}, h^{\prime}, r\right) \text { with }\left|u^{\prime}, h^{\prime}, r\right| \text { small and }(p, \zeta) \\
& \quad \text { in a conic neighborhood of }(\underline{p}, \underline{\hat{\zeta}}),
\end{aligned}
$$

where $\hat{\zeta} \in \bar{S}_{+}^{d} \cap\{0 \leqslant \hat{\gamma} \leqslant \delta\}$ and $\underline{p}$ is a fixed but arbitrary point of $\mathcal{C}$ (recall Remark 3.21).

So first we extend these symbols to all $(z, q, \zeta)$ as elements of $\Gamma^{0}, \Gamma^{1}$, and $\Gamma^{0}$ respectively so that $\mathcal{V}^{-1} \in \Gamma^{0}$; the equality (4.105) still holds just for $(z, q, \zeta)$ satisfying (4.106). Set $\mathcal{V}^{\varepsilon}=\mathcal{V}\left(\frac{x}{\varepsilon}, q^{\varepsilon}, \zeta\right) \in \Gamma_{1}^{0}$ and define the symbols $\mu_{k}^{\varepsilon} \in \Gamma_{1}^{1}$ and $R_{k}^{\varepsilon} \in \Gamma_{1}^{0}$ similarly.

Define $w=T_{\mathcal{V}^{-1}} \mathbf{v}_{1}$ and observe that the homogeneous calculus gives

$$
\mathrm{v}_{1}=T_{\mathcal{V}} w+\varepsilon r_{-1} \mathrm{v}_{1} .
$$

A short computation using (4.107), Eq. (4.104), and the homogeneous calculus shows $w$ satisfies the following problem:

$$
\begin{aligned}
\partial_{x} w & =\frac{1}{\varepsilon} T_{\mathcal{V}^{-1}} T_{\hat{g}^{11}} T_{\mathcal{V}} w+T_{\mathcal{V}^{-1}} H_{1}+T_{\partial_{x} \mathcal{V}^{-1}} T_{\mathcal{V}} w+r_{0} \mathrm{v}_{1} \\
& =\frac{1}{\varepsilon} T_{\mathcal{V}^{-1} \hat{g}^{11} \mathcal{V}} w+T_{\left(\partial_{x} \mathcal{V}^{-1}\right) \mathcal{V}} w+T_{\mathcal{V}^{-1}} H_{1}+r_{0} w+r_{0} \mathrm{v}_{1}
\end{aligned}
$$

Let us use $e_{0}$ to denote a symbol in $\Gamma_{1}^{0}$, whose precise identity may change from term to term, such that for $\theta>0$ as in (2.42):

$$
e_{0}=e_{0}\left(\frac{x}{\varepsilon}, t, y, x, \zeta\right) \quad \text { with }\left|\partial_{t, y}^{\beta} \partial_{\zeta}^{\alpha} e_{0}\right| \lesssim e^{-\theta \frac{|x|}{\varepsilon}}\langle\zeta\rangle^{-|\alpha|} \text { for }|\beta| \leqslant 1
$$

Then we have

$$
T_{\left(\partial_{x} \mathcal{V}^{-1}\right) \mathcal{V}}=\frac{1}{\varepsilon} T_{e_{0}}+r_{0}
$$

By (4.105), (4.106), and Remark 4.25,

(4.111) $\mathcal{V}^{-1} \hat{g}^{11} \mathcal{V}=\Lambda^{\varepsilon} \quad$ for $\varepsilon+|x|$ small, $\zeta \in \operatorname{supp}\left(\beta_{1} \chi_{H}\right)$, and $p(t, y)$ near $\underline{p}$.

Remark 4.29 (Another reduction). - As noted in Remark 4.7, we can suppose $U$ and $\psi$ are supported in a small neighborhood of a basepoint $(\underline{t}, \underline{y}, 0)$ such that $p(\underline{t}, \underline{y})=\underline{p}$. Let $\phi(t, y, x)$ be a smooth cutoff supported near $(\underline{t}, \underline{y}, 0)$ such that

$$
\phi U=U \text { and } \phi(\kappa \psi)=\kappa \psi
$$

for $\kappa(x)$ as in (4.14). Use of the calculus and the paraproduct estimate of Proposition 4.15 shows the estimates (4.102) follow from the same estimates when $\mathbb{V}$ is replaced by $T_{\phi} \mathbb{V}$; only absorbable errors are introduced by commuting $T_{\phi}$ through (4.101). Indeed, we have

$$
\left[T_{\phi}, \partial_{x}\right] \mathbb{V}=r_{0} \mathbb{V}, \quad \frac{1}{\varepsilon}\left[T_{\phi}, T_{g_{b}}\right] \mathbb{V}=\left(\begin{array}{c}
r_{0} \mathrm{v}_{1} \\
0 \\
\frac{1}{\varepsilon}\left[T_{\phi}, T_{g^{32}}\right] \mathrm{v}_{2}+r_{0} \mathrm{v}_{3}
\end{array}\right),
$$


where, because $g^{32}$ has the structure (4.52),

$$
\frac{1}{\varepsilon}\left\|\left[T_{\phi}, T_{g^{32}}\right] \mathrm{v}_{2}\right\|_{\Lambda_{\varepsilon}^{-1}} \lesssim\left\|\mathrm{v}_{2}\right\| .
$$

Thus, the components of $\mathcal{H}$ in (4.101) are modified only by absorbable terms.

Recalling the definition of $\mathbb{V}$ and using $\phi U=U$, we also have

$$
\left\|T_{\phi} \mathbb{V}-\mathbb{V}\right\|_{\Lambda_{\varepsilon}} \lesssim\left\|T_{\phi} \mathbb{V}-\mathbb{V}\right\|_{\langle\varepsilon \zeta\rangle} \lesssim \varepsilon\|U\|+\left|\psi_{m h}\right|
$$

for $\langle\varepsilon \zeta\rangle$ as in (4.40), so estimates for $T_{\phi} \mathbb{V}$ imply estimates for $\mathbb{V}$. Similarly, a partition of unity in $\zeta$ allows us to replace $T_{\phi} \mathbb{V}$ by $T_{\phi \tilde{\chi}} \mathbb{V}$, where $\tilde{\chi}$ is supported in a conic neighborhood of a basepoint $\hat{\zeta}$ as in (4.106). In the remainder of the proof of Proposition 4.28 we therefore suppose that $\mathbb{V}$ is replaced by $T_{\phi \tilde{\chi}} \mathbb{V}$ in (4.101) and (4.102), and we write the components of the latter again as $\mathrm{v}_{i}$. That is,

$$
\left(\mathrm{v}_{1}, \mathrm{v}_{2}, \mathrm{v}_{3}\right)=T_{\phi \tilde{\chi}} T_{\mathcal{W}^{-1}} V=T_{\phi \tilde{\chi}} T_{\mathcal{W}^{-1}}\left(T_{\beta_{1} \chi_{H}} U-T_{S^{\varepsilon} \beta_{1} \chi_{H}} \frac{\psi_{m h}}{\varepsilon}\right) .
$$

In view of (4.111) and this remark, an application of the calculus gives in place of (4.108):

$$
\partial_{x} w=\frac{1}{\varepsilon}\left(T_{\Lambda}+T_{e_{0}}\right) w+T_{\mathcal{V}^{-1}} H_{1}+r_{0} u_{1}+r_{0} \frac{\psi_{m h}}{\varepsilon} .
$$

With $R_{k}^{ \pm \infty}=\left.\left(\lim _{z \rightarrow \pm \infty} R_{k}(z, q, \zeta)\right)\right|_{q=q^{\varepsilon}}$ we have $R_{k \pm}^{\varepsilon}-R_{k}^{ \pm \infty}=e_{0, k \pm}$. Thus, setting

$$
w=\left(\mathrm{w}_{1}, \ldots, \mathrm{w}_{k_{0}}\right) \quad \text { and } T_{\mathcal{V}^{-1}} H_{1}=\left(\mathrm{h}_{1}, \ldots, \mathrm{h}_{k_{0}}\right)
$$

we can write the problem satisfied by $w_{1}$ as

$$
\partial_{x} \mathrm{w}_{1}=\frac{1}{\varepsilon}\left(T_{\mu_{1}}+T_{R_{1}^{ \pm \infty}}+T_{e_{0,1 \pm}}\right) \mathrm{w}_{1}+\mathrm{h}_{1}+\frac{1}{\varepsilon} \sum_{k=2}^{k_{0}} T_{e_{0 \pm}} \mathrm{w}_{k}+r_{0} u_{1}+r_{0} \frac{\psi_{m h}}{\varepsilon}
$$

parallel to (3.102). Now $e_{0,1 \pm}$ depends on both $R_{1}$ and $\left(\mathcal{V}, \mathcal{V}_{z}\right)$, while the $e_{0 \pm}$ on the right depend just on $\left(\mathcal{V}, \mathcal{V}_{z}\right)$.

Consider the case $-\operatorname{sgn}(\beta)=1$, where $\beta$ is any eigenvalue of $\bar{A}_{d}^{11}$. Letting $w^{ \pm}=\left.w\right|_{ \pm x \geqslant 0}$ we first estimate $w^{+}$. As in the frozen coefficient analysis (3.104), we need to introduce exponential weights. For a uniformly bounded weight function $\phi(z)$ to be chosen, set

$$
\omega=\left(\omega_{1}, \ldots, \omega_{k_{0}}\right):=e^{\phi\left(\frac{x}{\varepsilon}\right)} w^{+}=e^{\phi}\left(\mathrm{w}_{1}^{+}, \ldots, \mathrm{w}_{k_{0}}^{+}\right) .
$$

From (4.119) we have

$$
\begin{aligned}
\partial_{x} \omega_{1}= & \frac{1}{\varepsilon}\left(T_{\mu_{1}}+T_{R_{1}^{+\infty}}+T_{e_{0,1}}+\phi^{\prime}\right) \omega_{1}+e^{\phi} \mathrm{h}_{1} \\
& +\frac{1}{\varepsilon} \sum_{k=2}^{k_{0}} T_{e_{0}} \omega_{k}+r_{0} u_{1}+r_{0} \frac{\psi_{m h}}{\varepsilon}
\end{aligned}
$$

where $\phi^{\prime}=\phi_{z}\left(\frac{x}{\varepsilon}\right)$.

Denote the inner product in $L^{2}\left(\mathbb{R}_{t, y}^{d}\right)$ by $\left(\right.$, ). Pairing (4.121) with $\omega_{1}$ and integrating $\int_{0}^{\infty}$ yields

$$
\begin{aligned}
& \left|\omega_{1}(0)\right|^{2}+\frac{2}{\varepsilon} \Re \int_{0}^{\infty}\left(\left(T_{\mu_{1}}+T_{R_{1}^{+\infty}}\right) \omega_{1}, \omega_{1}\right)+\frac{2}{\varepsilon} \Re \int_{0}^{\infty}\left(\left(T_{e_{0,1}}+\phi^{\prime}\right) \omega_{1}, \omega_{1}\right) \\
& =-2 \Re \int_{0}^{\infty}\left(e^{\phi} \mathrm{h}_{1}, \omega_{1}\right)-\frac{2}{\varepsilon} \Re \int_{0}^{\infty} \sum_{k=2}^{k_{0}}\left(T_{e_{0}} \omega_{k}, \omega_{1}\right)-2 \Re \int_{0}^{\infty}\left(r_{0} u_{1}+r_{0} \frac{\psi_{m h}}{\varepsilon}, \omega_{1}\right) .
\end{aligned}
$$

$4^{e}$ SÉRIE - TOME $39-2006-\mathrm{N}^{\circ} 1$ 
Now the rule for adjoints gives for each $x$

$$
\begin{aligned}
\frac{1}{\varepsilon}\left(T_{e_{0}} \omega_{k}, \omega_{1}\right) & =\frac{1}{\varepsilon}\left(\omega_{k}, T_{e_{0}^{*}} \omega_{1}\right)+\left(\omega_{k}, r_{-1} \omega_{1}\right) \\
& \leqslant \frac{\alpha}{\varepsilon}\left|\omega_{k}\right|^{2}+\frac{C_{\alpha}}{\varepsilon}\left|T_{e_{0}^{*}} \omega_{1}\right|^{2}+C|\omega|^{2} .
\end{aligned}
$$

The right side of (4.122) is thus easily estimated by RHS $\leqslant$

$$
\begin{aligned}
& \delta_{1}\left(\gamma+\frac{1}{\varepsilon}\right)\left\|\omega_{1}\right\|^{2}+\frac{C_{\phi}}{\gamma+\frac{1}{\varepsilon}}\left(\left\|\mathrm{h}_{1}\right\|^{2}+\left\|u_{1}\right\|^{2}+\left|\psi_{m h} / \varepsilon\right|^{2}\right) \\
& +\frac{2 \alpha}{\varepsilon}\|\omega\|^{2}+\frac{C_{\alpha}}{\varepsilon}\left\|T_{e_{0}^{*}} \omega_{1}\right\|^{2}+C\|\omega\|^{2} .
\end{aligned}
$$

From Proposition 3.22 and (3.59) we have for $\varepsilon$ small, $0 \leqslant \gamma \leqslant \delta|\zeta|,|\zeta| \geqslant R$, and $(t, y, x, \zeta)$ in a conic neighborhood of $(\underline{t}, \underline{y}, 0, \underline{\hat{\zeta}})(\underline{\hat{\zeta}}$ as in (4.106))

$$
\begin{gathered}
\Re \mu_{1}^{\varepsilon}(t, y, x, \zeta)=\gamma \tilde{\mu}_{1} \quad \text { for } \tilde{\mu}_{1} \in \Gamma_{1}^{0} \text { such that } \tilde{\mu}_{1} \geqslant c>0, \\
\Re R_{1}^{\infty} \geqslant c>0 .
\end{gathered}
$$

Let $b(t, y, x, \zeta) \in \Gamma_{1}^{0}$ be a cutoff supported in the region where (4.125) holds with

$$
b(t, y, x, \zeta)=1 \quad \text { on } \operatorname{supp}(\phi) \times \operatorname{supp}\left(\tilde{\chi} \beta_{1} \chi_{H}\right)
$$

for $\phi, \tilde{\chi}$ as in (4.116). Let $(()$,$) denote the inner product on L^{2}\left(\overline{\mathbb{R}}_{+}^{d+1}\right)$. Then, since $T_{\gamma \tilde{\mu}_{1}}=$ $\varepsilon \gamma T_{\tilde{\mu}_{1}}$ and $\omega_{1}=T_{b} \omega_{1}+T_{1-b} \omega_{1}$, the calculus gives

$$
\begin{aligned}
& \left\|\omega_{1}\right\| \leqslant\left\|T_{b} \omega_{1}\right\|+C\left(\varepsilon\left\|u_{1}\right\|_{-1, \varepsilon, \gamma}+\varepsilon \frac{\left|\psi_{m h}\right|_{-1, \varepsilon, \gamma}}{\varepsilon}\right), \\
& \gamma \Re\left(\left(T_{\tilde{\mu}_{1}} T_{b} \omega_{1}, T_{b} \omega_{1}\right)\right) \\
& \quad \leqslant \frac{1}{\varepsilon} \Re\left(\left(T_{\gamma \tilde{\mu}_{1}} \omega_{1}, \omega_{1}\right)\right)+\gamma\left(\delta_{1}\left\|\omega_{1}\right\|^{2}+C_{\delta_{1}}\left(\varepsilon^{2}\left\|u_{1}\right\|_{-1, \varepsilon, \gamma}^{2}+\varepsilon^{2} \frac{\left|\psi_{m h}\right|_{-1, \varepsilon, \gamma}^{2}}{\varepsilon^{2}}\right)\right), \\
& \frac{1}{\varepsilon} \Re\left(\left(T_{R_{1}^{+\infty}} T_{b} \omega_{1}, T_{b} \omega_{1}\right)\right) \\
& \quad \leqslant \frac{1}{\varepsilon} \Re\left(\left(T_{R_{1}^{\infty}} \omega_{1}, \omega_{1}\right)\right)+\frac{1}{\varepsilon}\left(\delta_{1}\left\|\omega_{1}\right\|^{2}+C_{\delta_{1}}\left(\varepsilon^{2}\left\|u_{1}\right\|_{-1, \varepsilon, \gamma}^{2}+\varepsilon^{2} \frac{\left|\psi_{m h}\right|_{-1, \varepsilon, \gamma}^{2}}{\varepsilon^{2}}\right)\right) .
\end{aligned}
$$

Gårding's inequality and the first inequality of (4.127) imply the lower bounds

$$
\begin{gathered}
\gamma \Re\left(\left(T_{\tilde{\mu}_{1}} T_{b} \omega_{1}, T_{b} \omega_{1}\right)\right) \geqslant \frac{c \gamma}{2}\left\|\omega_{1}\right\|^{2}-\gamma C\left(\varepsilon^{2}\left\|u_{1}\right\|_{-1, \varepsilon, \gamma}^{2}+\left|\psi_{m h}\right|_{-1, \varepsilon, \gamma}^{2}\right), \\
\frac{1}{\varepsilon} \Re\left(\left(T_{R_{1}^{+\infty}} T_{b} \omega_{1}, T_{b} \omega_{1}\right)\right) \geqslant \frac{c}{2 \varepsilon}\left\|\omega_{1}\right\|^{2}-C\left(\varepsilon\left\|u_{1}\right\|_{-1, \varepsilon, \gamma}^{2}+\frac{\left|\psi_{m h}\right|_{-1, \varepsilon, \gamma}^{2}}{\varepsilon}\right) .
\end{gathered}
$$

Next we must deal with the $\frac{C_{\alpha}}{\varepsilon}\left\|T_{e_{0}^{*}} \omega_{1}\right\|^{2}$ term on the right in (4.124). The calculus gives

$$
\frac{C_{\alpha}}{\varepsilon}\left\|T_{e_{0}^{*}} \omega_{1}\right\|^{2} \leqslant \frac{C_{\alpha}}{\varepsilon} \Re\left(\left(T_{e_{0} e_{0}^{*}} \omega_{1}, \omega_{1}\right)\right)+C_{\alpha}\left\|\omega_{1}\right\|^{2} .
$$

Note that $e_{0} e_{0}^{*}$ decays like $e^{-2 \theta x / \varepsilon}$. Anticipating the analogues of (4.122) and (4.129) for the other $\omega_{k}$ and noting that $\alpha$ (small) and $C_{\alpha}$ can be fixed independently of $k$ ahead of time, we now choose $\phi(z)$ uniformly bounded on $[0, \infty)$ such that

$$
\Re\left(2\left(e_{0, k}+\phi^{\prime}\right)-C_{\alpha} e_{0} e_{0}^{*}\right) \geqslant e^{-\theta x / \varepsilon} \quad \text { for all } k .
$$


Now $T_{\phi^{\prime}} \omega_{1}=\phi^{\prime} \omega_{1}$ so an application of Gårding's inequality gives

$$
\frac{1}{\varepsilon} \Re\left(\left(\left(2\left(T_{e_{0,1}}+\phi^{\prime}\right)-C_{\alpha} T_{e_{0} e_{0}^{*}}\right) \omega_{1}, \omega_{1}\right)\right) \geqslant \frac{1}{2 \varepsilon} \int_{0}^{\infty} e^{-\theta x / \varepsilon}\left|\omega_{1}\right|^{2} d x-C \varepsilon\left\|\omega_{1}\right\|^{2} .
$$

Combining the estimates (4.124), (4.127), (4.128), and (4.131) we obtain from (4.122) by taking $\delta_{1}$ small:

$$
\begin{aligned}
& \left(\gamma+\frac{1}{\varepsilon}\right)\left\|\omega_{1}\right\|^{2}+\left|\omega_{1}(0)\right|^{2} \\
& \leqslant \frac{C_{\delta_{1}}}{\gamma+\frac{1}{\varepsilon}}\left(\left\|\mathrm{h}_{1}\right\|^{2}+\left\|u_{1}\right\|^{2}+\left|\psi_{m h} / \varepsilon\right|^{2}\right)+\left(\frac{C^{*} \alpha}{\varepsilon}+C_{\alpha}\right)\|\omega\|^{2} \\
& \quad+C\left(\gamma+\frac{1}{\varepsilon}\right)\left(\varepsilon^{2}\left\|u_{1}\right\|_{-1, \varepsilon, \gamma}^{2}+\left|\psi_{m h}\right|_{-1, \varepsilon, \gamma}^{2}\right),
\end{aligned}
$$

where $C^{*}$ depends on $c$ in (4.128). Adding (4.132) to similar estimates for the other components $\omega_{k}$ of $\omega$ and using the boundedness of $\phi$, we obtain for $\varepsilon$ small and provided $\alpha$ was fixed small enough:

$$
\left(\frac{1}{\varepsilon}+\gamma\right)\left\|w^{+}\right\|+\left(\frac{1}{\varepsilon}+\gamma\right)^{1 / 2}\left|w^{+}(0)\right| \leqslant C\left(\left\|H_{1}^{+}\right\|+\left\|u_{1}\right\|+\frac{\left|\psi_{m h}\right|}{\varepsilon}\right) .
$$

A similar argument on $x \leqslant 0$ yields

$$
\left(\frac{1}{\varepsilon}+\gamma\right)\left\|w^{-}\right\| \leqslant C\left(\left\|H_{1}^{-}\right\|+\left(\frac{1}{\varepsilon}+\gamma\right)^{1 / 2}\left|w^{-}(0)\right|+\left\|u_{1}\right\|+\frac{\left|\psi_{m h}\right|}{\varepsilon}\right) .
$$

Combining the estimates (4.133) and (4.134) using $w^{+}(0)=w^{-}(0)$ and recalling $\mathrm{v}_{1}=$ $T_{\mathcal{V}} w+\varepsilon r_{-1} \mathrm{v}_{1}$, we finally obtain the first estimate of (4.102) in the case $-\operatorname{sgn}(\beta)=1$. The other case is done the same way.

3. The parabolic block. We now complete the proof of Proposition 4.28 by proving the second of the estimates (4.102) for $\mathrm{v}^{\prime}:=\left(\mathrm{v}_{2}, \mathrm{v}_{3}\right)$ as modified in Remark 4.29 and satisfying:

$$
\partial_{x} \mathrm{v}^{\prime}=\frac{1}{\varepsilon} T_{g_{p}} \mathrm{v}^{\prime}+\left(\begin{array}{c}
H_{2} \\
H_{3}
\end{array}\right), \quad\left[\mathrm{v}^{\prime}\right]=0 .
$$

To get optimal estimates we need to switch now to the parabolic calculus. Since

$$
\frac{1}{\varepsilon}\left\|\left(T_{g^{32}}-g^{32}\right) \mathrm{v}_{2}\right\|_{\Lambda_{\varepsilon}^{-1}}+\frac{1}{\varepsilon}\left\|\left(T_{g^{33}}-g^{33}\right) \mathrm{v}_{3}\right\|_{\Lambda_{\varepsilon}^{-1}} \lesssim\left\|\mathrm{v}_{2}\right\|+\left\|\mathrm{v}_{3}\right\|
$$

by Lemma 4.19 and Proposition 4.15, and

$$
\frac{1}{\varepsilon}\left\|\left(P_{g^{32}}-g^{32}\right) \mathrm{v}_{2}\right\|_{\Lambda_{\varepsilon}^{-1}}+\frac{1}{\varepsilon}\left\|\left(P_{g^{33}}-g^{33}\right) \mathrm{v}_{3}\right\|_{\Lambda_{\varepsilon}^{-1}} \lesssim\left\|\mathrm{v}_{2}\right\| \Lambda_{\varepsilon}+\left\|\mathrm{v}_{3}\right\|
$$

by Proposition 4.16, we see that $v^{\prime}$ satisfies (4.135) if and only if it satisfies

$$
\partial_{x} \mathrm{v}^{\prime}-\frac{1}{\varepsilon} P_{g_{p}} \mathrm{v}^{\prime}=\left(\begin{array}{c}
H_{2} \\
H_{3}
\end{array}\right)+\left(\begin{array}{c}
0 \\
h_{3}
\end{array}\right):=\left(\begin{array}{c}
H_{2}^{\prime} \\
H_{3}^{\prime}
\end{array}\right), \quad\left[\mathrm{v}^{\prime}\right]=0 .
$$

with

$$
\left\|h_{3}\right\|_{\Lambda_{\varepsilon}^{-1}} \lesssim\left\|\mathrm{v}_{2}\right\|_{\Lambda_{\varepsilon}}+\left\|\mathrm{v}_{3}\right\| .
$$

Next set $\mathrm{v}^{\prime \prime}=P_{\Lambda^{-1}} \mathrm{v}^{\prime}$ and commute $P_{\Lambda^{-1}}$ through (4.138) using the parabolic calculus to see that

$4^{\mathrm{e}}$ SÉRIE - TOME $39-2006-\mathrm{N}^{\circ} 1$ 


$$
\partial_{x} \mathrm{v}^{\prime \prime}-\frac{1}{\varepsilon} P_{g_{p}} \mathrm{v}^{\prime \prime}=P_{\Lambda^{-1}}\left(\begin{array}{c}
H_{2}^{\prime} \\
H_{3}^{\prime}
\end{array}\right)+\left(\begin{array}{c}
0 \\
r_{0} \mathrm{v}_{2}^{\prime}+r_{-1} \mathrm{v}_{3}^{\prime}
\end{array}\right):=\left(\begin{array}{c}
H_{2}^{\prime \prime} \\
H_{3}^{\prime \prime}
\end{array}\right)
$$

where $r_{0}$ and $r_{-1}$ have orders 0 and -1 on the $P \mathcal{H}^{s}$ spaces now.

Let $\underline{p}=p(\underline{t}, \underline{y})$ be a basepoint such that for $z \in \mathbb{R},|\zeta|$ large, and $q$ near $q=(\underline{p}, 0,0,0)$ we have a conjugator $V$ as in (3.115) and symmetrizers $S^{ \pm}$as in (3.117). As before we can suppose that $U$ and $\psi$ are supported near $(\underline{t}, \underline{y}, 0)$ and we let $\phi$ be a cutoff as in (4.112). Choose smooth cutoffs $\phi^{\prime}(t, y, x)$ and $\chi^{\prime}(\zeta)$ supported, respectively, near $(\underline{t}, \underline{y}, 0)$ and in $|\zeta| \geqslant R$, such that

$$
\phi^{\prime} \phi=\phi, \quad \chi^{\prime} \chi_{H}=\chi_{H} .
$$

If we set $\mathrm{v}^{\prime \prime \prime}=P_{\phi^{\prime} \chi^{\prime}} \mathrm{v}^{\prime \prime}$, then we can apply the high frequency estimate of [30], Proposition 4.6, to conclude for large enough $R$ that

$$
\begin{aligned}
& \frac{1}{\varepsilon}\left\|\mathrm{v}_{2}^{\prime \prime \prime}\right\|_{\Lambda_{\varepsilon}^{2}}+\frac{1}{\varepsilon}\left\|\mathrm{v}_{3}^{\prime \prime \prime}\right\|_{\Lambda_{\varepsilon}}+\frac{1}{\sqrt{\varepsilon}}\left|\mathrm{v}_{2}^{\prime \prime \prime}(0)\right|_{\Lambda_{\varepsilon}^{3 / 2}}+\frac{1}{\sqrt{\varepsilon}}\left|\mathrm{v}_{3}^{\prime \prime \prime}(0)\right|_{\Lambda_{\varepsilon}^{1 / 2}} \\
& \quad \lesssim\left\|H_{2}^{\prime \prime}\right\|_{\Lambda_{\varepsilon}}+\left\|H_{3}^{\prime \prime}\right\|+\left\|\mathrm{v}_{2}^{\prime \prime}\right\|\left\|_{\Lambda_{\varepsilon}}+\right\| \mathrm{v}_{3}^{\prime \prime} \|+\left|\mathrm{v}_{2}^{\prime \prime}(0)\right|_{\Lambda_{\varepsilon}}+\left|\mathrm{v}_{3}^{\prime \prime}(0)\right|
\end{aligned}
$$

We remark that the proposition cited here is a quantized version of the frozen coefficient estimate given for the parabolic block in Section 3.2.

Using (4.140) and the definition of $\mathrm{v}^{\prime}$ (4.116), we see that the right side of (4.142) is $\lesssim$

$$
\left\|H_{2}^{\prime}\right\|+\left\|H_{3}^{\prime}\right\|_{\Lambda_{\varepsilon}^{-1}}+\left\|u_{1}, u_{2}, u_{3}\right\|+\left|u_{1}(0), u_{2}(0), u_{3}(0)\right|+\left|\psi_{m h} / \varepsilon\right| .
$$

Finally, we need to estimate the error $v^{\prime \prime \prime}-v^{\prime \prime}$. Some care is needed because operators from both the homogeneous and parabolic calculi enter into the definition of $v^{\prime \prime \prime}$. We have

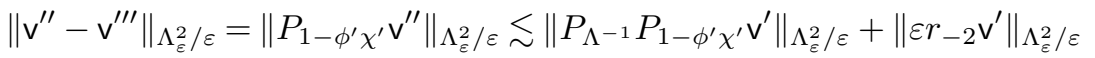

$$
\begin{aligned}
& \leqslant\left\|P_{\Lambda^{-1}}\left(P_{1-\phi^{\prime} \chi^{\prime}}-T_{1-\phi^{\prime} \chi^{\prime}}\right) \mathrm{v}^{\prime}\right\|_{\Lambda_{\varepsilon}^{2} / \varepsilon}+\| P_{\Lambda^{-1}} T_{1-\phi^{\prime} \chi^{\prime} \mathrm{v}^{\prime}\left\|_{\Lambda_{\varepsilon}^{2} / \varepsilon}+\right\| \mathrm{v}^{\prime} \|} \\
& =A+B+\left\|\mathrm{v}^{\prime}\right\| \text {. }
\end{aligned}
$$

The paraproduct estimates of Propositions 4.15 and 4.16 give $A \lesssim\left\|\mathrm{v}^{\prime}\right\|$. Recalling (4.116), (4.141) and using the homogeneous calculus, we get

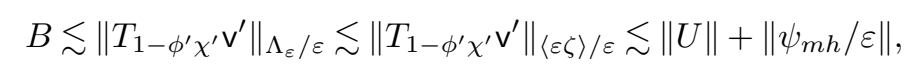

where $\langle\varepsilon \zeta\rangle$ is the homogeneous weight as in (4.40).

Combining this and similar estimates of $v^{\prime \prime \prime}-v^{\prime \prime}$ in terms of the other norms appearing on the left in (4.142) with (4.143), we find

$$
\begin{aligned}
& \frac{1}{\varepsilon}\left\|\mathrm{v}_{2}^{\prime \prime}\right\|_{\Lambda_{\varepsilon}^{2}}+\frac{1}{\varepsilon}\left\|\mathrm{v}_{3}^{\prime \prime}\right\|_{\Lambda_{\varepsilon}}+\frac{1}{\sqrt{\varepsilon}}\left|\mathrm{v}_{2}^{\prime \prime}(0)\right|_{\Lambda_{\varepsilon}^{3 / 2}}+\frac{1}{\sqrt{\varepsilon}}\left|\mathrm{v}_{3}^{\prime \prime}(0)\right|_{\Lambda_{\varepsilon}^{1 / 2}} \\
& \quad \lesssim\left\|H_{2}^{\prime}\right\|+\left\|H_{3}^{\prime}\right\|_{\Lambda_{\varepsilon}^{-1}}+\text { a.t. }
\end{aligned}
$$

This is equivalent to the desired estimate for $\mathrm{v}^{\prime}=P_{\Lambda} \mathrm{v}^{\prime \prime}$.

This concludes the proof of Proposition 4.28 and thus also the proof of Proposition 4.23 in the case $\beta=\beta_{1}$.

4. The case $\beta=\beta_{2}$. Finally, we prove Proposition 4.23 in the case $\beta=\beta_{2}$, where $\beta_{2}$ is supported in $\gamma \geqslant \frac{\delta|\zeta|}{2}$ (recall (4.17)). Let $g_{h}^{11}$ be the principal part, homogeneous of degree one, of $g^{11}$. Then we can rewrite the problem (4.70)(a) equivalently as

$$
\begin{array}{r}
\partial_{x} v_{1}-\frac{1}{\varepsilon} T_{g_{h}^{11}} v_{1}=h_{1}^{\prime \prime}+\frac{1}{\varepsilon}\left(r_{0} v_{1}+T_{g^{12}} v_{2}+T_{g^{13}} v_{3}\right):=H_{1}, \quad\left[v_{1}\right]=0, \\
\partial_{x}\left(\begin{array}{c}
v_{2} \\
v_{3}
\end{array}\right)-\frac{1}{\varepsilon} T_{g_{p}}\left(\begin{array}{c}
v_{2} \\
v_{3}
\end{array}\right)=\left(\begin{array}{c}
0 \\
h_{2}^{\prime \prime}+\frac{1}{\varepsilon} T_{g^{31}} v_{1}
\end{array}\right):=H_{3}, \quad\left[v_{2}\right]=0,\left[v_{3}\right]=0 .
\end{array}
$$


We have the estimates

$$
\begin{aligned}
& \frac{1}{\varepsilon}\left(\left\|r_{0} v_{1}\right\|+\left\|T_{g^{12}} v_{2}\right\|+\left\|T_{g^{13}} v_{3}\right\|\right) \lesssim \frac{1}{\varepsilon}\left(\left\|v_{1}\right\|+\left\|v_{2}\right\|_{\Lambda_{\varepsilon}}+\left\|v_{3}\right\|\right), \\
& \frac{1}{\varepsilon}\left\|T_{g^{31}} v_{1}\right\|_{\Lambda_{\varepsilon}^{-1}}=\frac{1}{\varepsilon}\left\|T_{\langle\varepsilon \zeta\rangle} r_{0} v_{1}\right\|_{\Lambda_{\varepsilon}^{-1}} \lesssim \frac{1}{\varepsilon}\left\|v_{1}\right\|_{\langle\varepsilon \zeta\rangle^{1 / 2}},
\end{aligned}
$$

since $\Lambda_{\varepsilon} \geqslant \sqrt{\langle\varepsilon \zeta\rangle}$. Thus, estimate (4.78) of Proposition 4.23 is implied directly by the following estimates for (4.147):

$$
\begin{aligned}
& \frac{1}{\varepsilon}\left\|v_{1}\right\|_{\langle\varepsilon \zeta\rangle}+\frac{1}{\sqrt{\varepsilon}}\left|v_{1}(0)\right|_{\langle\varepsilon \zeta\rangle^{1 / 2}} \lesssim\left\|H_{1}\right\|+\text { a.t. } \\
& \frac{1}{\varepsilon}\left\|v_{2}\right\|_{\Lambda_{\varepsilon}}+\frac{1}{\varepsilon}\left\|v_{3}\right\|+\frac{1}{\sqrt{\varepsilon}}\left|v_{2}(0)\right|_{\Lambda_{\varepsilon}^{1 / 2}}+\frac{1}{\sqrt{\varepsilon}}\left|v_{3}(0)\right|_{\Lambda_{\varepsilon}^{-1 / 2}} \lesssim\left\|H_{3}\right\|_{\Lambda_{\varepsilon}^{-1}}+\text { a.t. }
\end{aligned}
$$

Let $\phi(t, y, x)$ be a smooth cutoff supported near $(\underline{t}, \underline{y}, 0)$ with $p(\underline{t}, \underline{y})=\underline{p}$, a fixed but arbitrary point of $\mathcal{C}$. Choose also a smooth cutoff $\chi(\zeta)$ supported in $|\zeta| \geqslant R$ with $\chi \bar{\chi}_{H}=\chi_{H}$. As long as

$$
\phi U=U \quad \text { and } \quad \phi \kappa(x) \psi=\kappa(x) \psi,
$$

we can again make the reduction of Remark 4.29 and replace $V$ by $T_{\phi \chi} V$.

The second estimate in (4.149) is proved exactly like the earlier estimate for the parabolic block (4.135).

For the estimate of $v_{1}$ we use symmetrizers associated to symbols constructed from $S^{ \pm}(z, q, \hat{\zeta})$ as in (3.132). The symbols $S^{ \pm}$are defined for $q$ near $q=(p, 0,0,0)$, so we choose cutoffs $\phi_{1}(t, y, x)$ and $\chi_{1}(\zeta)$ supported, respectively, near $(\underline{t}, \underline{y}, 0)$ and in $|\zeta| \geqslant R / 2$ such that

$$
\phi_{1} \phi=\phi, \quad \chi_{1} \chi=\chi,
$$

and define self-adjoint symbols $\sigma_{ \pm}^{\varepsilon}(t, y, x, \zeta) \in \Gamma_{1}^{1}$ by

$$
\sigma^{\varepsilon}=\phi_{1}(t, y, x) \chi_{1}(\zeta)\langle\zeta\rangle S^{ \pm}\left(\frac{x}{\varepsilon}, q^{\varepsilon}, \hat{\zeta}\right)
$$

We have for $U^{ \pm} \in \mathbb{C}^{N-s}$ and $(t, y, x, \zeta) \in \operatorname{supp}(\phi) \times \operatorname{supp}(\chi)$ :

$$
\begin{gathered}
\Re\left(\sigma_{ \pm}^{\varepsilon} g_{h}^{11} U^{ \pm}, U^{ \pm}\right) \geqslant\langle\zeta\rangle^{2}|U|^{2} \\
\left(\sigma_{+}^{\varepsilon} U^{+}, U^{+}\right)-\left(\sigma_{-}^{\varepsilon} U^{-}, U^{-}\right)+C\langle\zeta\rangle\left|\Gamma\left(U^{+}, U^{-}\right)\right|^{2} \geqslant C\langle\zeta\rangle|U|^{2} \quad \text { on } x=0 .
\end{gathered}
$$

Taking as Kreiss symmetrizers the self-adjoint operators

$$
\mathcal{S}^{ \pm}=\Re T_{\sigma_{ \pm}^{\varepsilon}}=\frac{1}{2}\left(T_{\sigma_{ \pm}^{\varepsilon}}+\left(T_{\sigma_{ \pm}^{\varepsilon}}\right)^{*}\right),
$$

we can now repeat the proof of Proposition 4.6 of [30], which uses properties (4.153) and Gårding's inequality, to obtain the first estimate of (4.149). This argument is a quantized version of the frozen coefficient argument given in Section 3.2.

This concludes the proof of Proposition 4.23 and therefore also the proof of the variable coefficient, high frequency estimates of Theorem 4.4.

\subsection{Low and medium frequency estimates}

In Section 3.3 we have discussed the low and medium frequency estimates (3.6), (3.7) for the frozen coefficient linearized problem, assuming that the $r$ component of $q=\left(p, u^{\prime}, h^{\prime}, r\right)$ is zero (recall Remark 3.32). The corresponding variable coefficient estimates near the front, (4.19) 
and (4.20), can be proved by quantizing the arguments in the frozen case with semiclassical paradifferential operators, provided we show that the perturbation $r_{\varepsilon} \partial_{x} v^{1}$ is negligible in the proof of those estimates. For this we need to work with the paradifferential form of the problem (4.57):

$$
\begin{aligned}
& \partial_{x} U-\frac{1}{\varepsilon} T_{g^{\varepsilon}} U=\left(\begin{array}{c}
h_{1}^{\prime} \\
0 \\
h_{2}^{\prime}
\end{array}\right)+\frac{1}{\varepsilon}\left(\begin{array}{c}
T_{n_{1}^{\varepsilon}} \nabla_{\gamma} \phi \\
0 \\
T_{n_{2}^{\varepsilon}} \nabla_{\gamma} \phi
\end{array}\right), \\
& {[U]=0, \quad\left(\partial_{t}+\gamma\right) \psi-\varepsilon \triangle_{y} \phi+\ell\left(q^{\varepsilon}\right) \cdot v_{2}=e \quad \text { on } x=0,}
\end{aligned}
$$

where $U=\left(u_{1}, u_{2}, \varepsilon \partial_{x} u_{2}\right)$. We define $\tilde{r}_{\varepsilon} \in \Gamma_{2}^{1}$ and $g_{0}^{\varepsilon}$ and by

$$
T_{g^{\varepsilon}}=T_{g_{0}^{\varepsilon}}+\left(\begin{array}{ccc}
0 & 0 & 0 \\
0 & 0 & 0 \\
T_{b_{d} r_{\varepsilon} g^{11}} & T_{b_{d} r_{\varepsilon} g^{12}} & T_{b_{d} r_{\varepsilon} g^{13}}
\end{array}\right)=T_{g_{0}^{\varepsilon}}+T_{\tilde{r}_{\varepsilon}},
$$

where $b_{d}:=\left(\widetilde{B}_{d, d}^{22}\right)^{-1}\left(\frac{x}{\varepsilon}, q^{\varepsilon}\right)$. For low and medium frequency cutoffs $\chi_{L}(\zeta), \chi_{M}(\zeta)$ as in (4.15), set $\chi_{l m}=\chi_{L}+\chi_{M}$, apply $T_{\chi_{l m}}$ to (4.155) and then shift

$$
\frac{1}{\varepsilon} T_{\chi_{l m}} T_{\tilde{r}_{\varepsilon}} U
$$

to the right as a new forcing term. Since $\frac{1}{\varepsilon} \chi_{l m} \tilde{r}_{\varepsilon}$ is bounded in $\Gamma_{2}^{0}$, the term (4.157) can be absorbed by the left side of the composite estimate (4.11); thus, it is an "a.t." term.

It remains to prove the estimates (4.19), (4.20) for the problem (4.155) with $g_{0}^{\varepsilon}$ in place of $g^{\varepsilon}$. This part of the analysis is essentially identical to arguments in [12]. We refer to [12, Appendix A.2] for the low frequency estimate and to [12, Section 5.4] or [30, Section 4.4] for the medium frequency estimate.

Remark 4.30. - (1) If the perturbation $r_{\varepsilon} \partial_{x} v^{1}$ were not negligible in the low and medium frequency regimes, we would have to incorporate it into the main operator; but then it would, for example, destroy hyperbolicity (H4), which is needed for the low frequency analysis.

(2) Note that $r_{\varepsilon} \partial_{x} v^{1}$ is not negligible in the proof of the high frequency estimate. The weight $\Lambda_{\varepsilon}^{-1}$ on $\left\|f_{2}\right\|_{\Lambda_{\varepsilon}^{-1}}$ in (4.21) can be used to absorb the contributions from $T_{b_{d} r_{\varepsilon} g^{12}}$ and $T_{b_{d} r_{\varepsilon} g^{13}}$ in (4.156), but it only "absorbs half" of the $t$ derivative in the contribution from $T_{b_{d} r_{\varepsilon}} g^{11}$. Fortunately, (H4) is not used in the high frequency estimate.

\subsection{Estimates away from the front}

THEOREM 4.31 (Estimate away from the front). - Assume (H0)-(H7) and (H10), and let $\kappa_{0}>0$ be as in Theorem 4.3. Assume $u^{ \pm}, f^{ \pm}$are $C^{\infty}$ with compact support on $\overline{\mathbb{R}}_{ \pm}^{d+1} \cap$ $\left\{|x| \geqslant \kappa_{0}\right\}, \psi \in C_{0}^{\infty}\left(\mathbb{R}^{d}\right)$, and $(u, \psi)$ satisfies (4.2). Then $\psi=0$ and there exist $\gamma_{0}$, $\varepsilon_{0}$ such that for $\gamma \geqslant \gamma_{0}, 0<\varepsilon \leqslant \varepsilon_{0}$ we have:

$$
\gamma\|u\|+\sqrt{\gamma \varepsilon}\left\|\partial_{x, y} u_{2}\right\| \leqslant C\|f\| .
$$

Remark 4.32. - (1) The extra boundary condition in the transmission problem (4.2) and the fact that $u$ vanishes near $x=0$ imply $\psi=0$. Thus, we just need to consider $u$ with support as above and satisfying

$$
\frac{1}{\varepsilon} \mathcal{L}\left(\frac{x}{\varepsilon}, q^{\varepsilon}, \varepsilon D_{t}, \varepsilon \gamma, \varepsilon D_{y}, \varepsilon \partial_{x}\right) u=f
$$


(2) Observe that away from the front, the $u_{\varepsilon}^{\prime}$ component of $q^{\varepsilon}$ as in (4.3) is no longer necessarily small. On the other hand we do assume $W\left(\frac{x}{\varepsilon}, q^{\varepsilon}\right) \in \mathcal{U}^{*}$ and $W\left(\frac{x}{\varepsilon}, q^{\varepsilon}\right) \in \mathcal{U}$ for $\left|\frac{x}{\varepsilon}\right|$ large ((H10), part (f)).

The proof of Theorem 4.31 has much in common with the proof of Theorem 4.3, but is much simpler since there are no boundary conditions, no glancing modes, and formerly singular terms like $\frac{1}{\varepsilon} E\left(\frac{x}{\varepsilon}, q^{\varepsilon}\right) u$ are now negligible. The Evans hypothesis (H9) is now irrelevant, there is no need to write the problem as a first order system, and the only part of Section 3.2 that is needed is Section 3.2.2.

The proof of the estimate away from the boundary in [30, Section 5.3] for the fully parabolic case can be used here almost verbatim, but there are a couple of new points in the symmetrizer construction for the partially parabolic case that we now discuss.

The symmetrizers are given by matrix symbols $s^{\varepsilon}\left(t, y, x, \eta, \xi_{d}\right)$. Set $\xi=\left(\eta, \xi_{d}\right)$. The low, medium, and large frequency regimes are defined by

$$
|\varepsilon \xi| \leqslant \rho_{0}, \quad \rho_{0} \leqslant|\varepsilon \xi| \leqslant R, \quad R \leqslant|\varepsilon \xi|
$$

respectively.

In the low and medium frequency regimes, our strict dissipativity condition (H5) is equivalent to the condition in the strictly parabolic case where the right side of (2.16) is replaced by $c|\xi|^{2}$. Thus, the symmetrizer construction of [30, Proposition 5.6] applies without change for bounded frequencies, provided we check that the perturbation $r_{\varepsilon} \partial_{x} v^{1}$, which is present now but not in [30], is negligible for bounded frequencies. Indeed, $\varepsilon \xi_{d}$ is bounded so $\left\|r_{\varepsilon} \partial_{x} u^{1}\right\|$ can be absorbed by the left side of (4.158).

The main new point is in the high frequency regime where one should first conjugate to block form as in (3.20). The symmetrizer for the $\widehat{B}^{22}$ block may now be constructed just as in the high frequency argument of [30, Proposition 5.6]. After another conjugation of the $\widehat{A}^{11}$ block as in (3.28), with blocks $C_{k}^{11}$ satisfying (3.29), a symmetrizer $s^{11}=\operatorname{diag}\left(s_{k}^{11}\right)$ for the (11) block is easily constructed satisfying

$$
\begin{gathered}
s_{k}^{11}=\left(s_{k}^{11}\right)^{*}, \quad s_{k}^{11} \geqslant I_{r_{k}}, \\
\Re\left(s_{k}^{11} C_{k}^{11}\right) \geqslant I_{r_{k}} .
\end{gathered}
$$

With these symmetrizers the estimate (4.158) follows in the usual way.

Remark 4.33. - The argument we have given in the high frequency regime uses the observation made in Remark 3.13 that the conjugations of Section 3.2.2 are valid for profiles $W(z, q)$ satisfying only conditions (a), (b) of Definition 2.16 . The parameter $q$ is also allowed to have a nonzero $r$ component in Section 3.2.2.

\section{Approximate solutions}

In this section we adapt the construction of high order approximate solutions in [12,17] for the case of positive viscosity to the partially parabolic case considered in this paper. A precise statement of the properties of the approximate solutions is given in Proposition 5.7.

We seek an approximate solution $\left(u_{\varepsilon}^{a}, \psi_{\varepsilon}^{a}\right)$ to the $N \times N$ system (1.1)

$$
\sum_{j=0}^{d} \partial_{j} f_{j}(u)-\varepsilon \sum_{j, k=1}^{d} \partial_{j}\left(B_{j, k}(u) \partial_{k} u\right)=0,
$$

given a shock solution $\left(u^{0}, \psi^{0}\right)$ to the associated hyperbolic system.

$4^{e}$ SÉRIE - TOME $39-2006-\mathrm{N}^{\circ} 1$ 
As before we introduce the unknown front $x=\psi_{\varepsilon}(t, y)$, change variables $\tilde{x}=x-\psi_{\varepsilon}(t, y)$, drop tildes and epsilons, and rewrite (5.1) in our usual notation (2.20)

$$
\sum_{j=0}^{d-1} A_{j}(u) \partial_{j} u+\widetilde{A}_{d}(u, d \psi) \partial_{d} u-\varepsilon \sum_{j, k=1}^{d} \partial_{j}\left(\widetilde{B}_{j, k}(u, d \psi) \partial_{k} u\right)=0 .
$$

We are also given a leading profile $\mathcal{U}^{0}(t, y, x, z)$, which in the new coordinates is given in $\pm x \geqslant 0$ by

$$
\mathcal{U}^{0}\left(t, y, x, \frac{x}{\varepsilon}\right)=W_{0}\left(\frac{x}{\varepsilon}, p(t, y)\right)+u^{0}\left(t, y, x+\psi^{0}(t, y)\right)-u^{0}\left(t, y, \psi^{0}(t, y)\right) .
$$

In particular we have

$$
\mathcal{U}^{0}(t, y, 0, z)=W_{0}(z, p(t, y)), \quad \mathcal{U}^{0}=\left(\mathcal{U}^{0,1}, \mathcal{U}^{0,2}\right) .
$$

Recall that we view (5.2) as representing two problems for $(u, \psi)$, one on $x \geqslant 0$ and one on $x \leqslant 0$ with transmission boundary conditions

$$
[u]=0, \quad\left[\partial_{x} u_{2}\right]=0 \quad \text { on } x=0 .
$$

We add the extra boundary condition on $\{x=0\}$ :

$$
\partial_{t} \psi-\varepsilon \triangle_{y} \psi+\left.\ell(t, y) \cdot u_{2}\right|_{x=0}=\partial_{t} \psi^{0}-\varepsilon \triangle_{y} \psi^{0}+\ell(t, y) \cdot \mathcal{U}^{0,2}(t, y, 0,0)
$$

where $\ell(t, y)$ has been chosen so that

$$
\ell(t, y) \cdot \partial_{z} \mathcal{U}^{0,2}(t, y, 0,0)>0 .
$$

We seek an approximate solution $\left(u_{\varepsilon}^{a}, \psi_{\varepsilon}^{a}\right)$ of the form (dropping epsilons)

$$
\begin{gathered}
\psi^{a}=\psi^{0}(t, y)+\varepsilon \psi^{1}(t, y)+\cdots+\varepsilon^{M} \psi^{M}(t, y), \\
u^{a}=\left.\left(\mathcal{U}^{0}(t, y, x, z)+\varepsilon \mathcal{U}^{1}(t, y, x, z)+\cdots+\varepsilon^{M} \mathcal{U}^{M}(t, y, x, z)\right)\right|_{z=\frac{x}{\varepsilon}},
\end{gathered}
$$

where

$$
\mathcal{U}^{j}(t, y, x, z)=U^{j}(t, y, x)+V^{j}(t, y, z)
$$

$U_{ \pm}^{0}(t, y, x)=\left.u^{0}\left(t, y, x+\psi^{0}(t, y)\right)\right|_{ \pm x \geqslant 0}$, and the $V_{ \pm}^{j}(t, y, z)$ are boundary layer profiles exponentially decreasing to 0 as $z \rightarrow \pm \infty$.

\subsubsection{Interior profile equations}

We substitute (5.9) into (5.2) and write the result as

$$
\left.\sum_{-1}^{M} \varepsilon^{j} \mathcal{F}^{j}(t, y, x, z)\right|_{z=\frac{x}{\varepsilon}}+\varepsilon^{M} R^{\varepsilon, M}(t, y, x),
$$

where

$$
\mathcal{F}^{j}(x, z)=F^{j}(x)+G^{j}\left(x^{\prime}, z\right),
$$

and the $G^{j}$ decrease exponentially to 0 as $z \rightarrow \pm \infty$.

In writing out the $F^{j}, G^{j}$ we use the following notation. 
NOTATION 5.1.-

(1) $\tilde{f}_{d}(u, d \phi) \equiv f_{d}(u)-\sum_{0}^{d-1} f_{j}(u) \partial_{j} \phi$.

(2) $H\left(U^{0}, d \psi^{0}\right) \partial \equiv \sum_{0}^{d-1} A_{j}\left(U^{0}\right) \partial_{j}+\widetilde{A}_{d}\left(U^{0}, d \psi^{0}\right) \partial_{d}$.

(3) $d_{u} \widetilde{A}_{d}\left(\mathcal{U}^{0}, d \psi^{0}\right)(v, w) \equiv \sum_{1}^{N} v_{i} \partial_{u_{i}} \widetilde{A}_{d}\left(\mathcal{U}^{0}, d \psi^{0}\right) w=\partial_{u} \widetilde{A}_{d}\left(\mathcal{U}^{0}, d \psi^{0}\right)(w, v)$, by symmetry of Hessians.

(4) $B(u) d \phi \equiv-\sum_{0}^{d-1} A_{j}(u) \partial_{j} \phi$.

(5) $\mathbb{B}(u) d \phi \equiv-\sum_{0}^{d-1} f_{j}(u) \partial_{j} \phi$.

(6) $[h(u)] \equiv h\left(u_{+}\right)-h\left(u_{-}\right)$on $x=0$, where $u_{ \pm}$denote the limits from the right/left at $x_{d}=0$.

Next we recall our notation for viscosity matrices:

NOTATION 5.2.-

(1) Let $\nu=\left(\nu_{0}, \nu_{1}, \ldots, \nu_{d}\right)=\left(-\partial_{0} \psi, \ldots,-\partial_{d-1} \psi, 1\right), \nu^{0}=\left(-\partial_{0} \psi^{0}, \ldots,-\partial_{d-1} \psi^{0}, 1\right)$, and $\nu^{1}=\left(-\partial_{0} \psi^{1}, \ldots,-\partial_{d-1} \psi^{1}, 1\right)$.

(2) $\widetilde{B}_{j, k}(u)=B_{j, k}(u)$ if $j<d, k<d$.

(3) $\widetilde{B}_{j, d}(u, \nu)=\sum_{k=1}^{d} B_{j, k}(u) \nu_{k}$ if $j<d ; \widetilde{B}_{d, k}(u)=\sum_{j=1}^{d} B_{j, k}(u) \nu_{j}$ if $k<d$.

(4) $\widetilde{B}_{d, d}(u, \nu)=\sum_{j, k=1}^{d} B_{j, k}(u) \nu_{j} \nu_{k}$.

(5) $\widetilde{B}_{j, k}^{0}$ is defined just like $\widetilde{B}_{j, k}$, except that $\left(\mathcal{U}^{0}, \nu^{0}\right)$ is substituted for $(u, \nu)$.

(6) $d \widetilde{B}_{d, d}^{0}(v, w)=\sum_{1}^{N} v_{i} \partial_{u_{i}} \widetilde{B}_{d, d}^{0} w$.

The interior profile equations are obtained by setting the $F^{j}, G^{j}$ equal to zero. In the following expressions for $G^{j}(t, y, z)$, the functions $U^{j}(t, y, x)$ and their derivatives are evaluated at $(t, y, 0)$. We have

$$
\begin{aligned}
& F^{-1}(t, y, x)=0 \\
& G^{-1}(t, y, z)=-\partial_{z}\left(\widetilde{B}_{d, d}^{0} \partial_{z} \mathcal{U}^{0}\right)+\partial_{z} \tilde{f}_{d}\left(\mathcal{U}^{0}, d \psi^{0}\right) \\
F^{0}(t, y, x)= & H\left(U^{0}, d \psi^{0}\right) \partial U^{0} \\
G^{0}(t, y, z)= & -\partial_{z}\left(\widetilde{B}_{d, d}^{0} \partial_{z} V^{1}\right)+\partial_{z}\left(\widetilde{A}_{d}\left(\mathcal{U}^{0}, d \psi^{0}\right)\left(U^{1}+V^{1}\right)\right. \\
& \left.-d \widetilde{B}_{d, d}^{0}\left(U^{1}+V^{1}, \partial_{z} V^{0}\right)+\mathbb{B}\left(\mathcal{U}^{0}\right) d \psi^{1}\right)+Q^{0}\left(U^{0}, V^{0}, d \psi^{0}, d \psi^{1}\right)
\end{aligned}
$$

where $Q^{0}=Q^{0}(t, y, z)$ (for short) is exponentially decaying in $z$. In fact

$$
\begin{aligned}
Q^{0}= & \sum_{0}^{d-1} A_{j}\left(\mathcal{U}^{0}\right) \partial_{j} V^{0} \\
& +\sum_{1}^{d-1}\left(A_{j}\left(\mathcal{U}^{0}\right)-A_{j}\left(U^{0}\right)\right) \partial_{j} U^{0}+\left(\widetilde{A}_{d}\left(\mathcal{U}^{0}, d \psi^{0}\right)-\widetilde{A}_{d}\left(U^{0}, d \psi^{0}\right)\right) \partial_{d} U^{0} \\
& -\left\{\sum_{j=1}^{d-1} \partial_{j}\left(\widetilde{B}_{j, d}^{0} \partial_{z} V^{0}\right)+\sum_{k=1}^{d-1} \partial_{z}\left(\widetilde{B}_{d, k}^{0} \partial_{k} \mathcal{U}^{0}\right)\right. \\
& +\sum_{j, k=1}^{d} \nu_{j}^{0} \nu_{k}^{0} \partial_{u} B_{j, k}^{0}\left(\partial_{z} V^{0}, \partial_{d} U^{0}\right)+\sum_{j, k=1}^{d} \nu_{j}^{0} \nu_{k}^{0} \partial_{u} B_{j, k}^{0}\left(\partial_{d} U^{0}, \partial_{z} V^{0}\right) \\
& \left.+\sum_{j, k=1}^{d} B_{j, k}^{0}\left(\nu_{j}^{0} \nu_{k}^{1}+\nu_{k}^{0} \nu_{j}^{1}\right) \partial_{z}^{2} V^{0}+\sum_{j, k=1}^{d}\left(\nu_{j}^{0} \nu_{k}^{1}+\nu_{k}^{0} \nu_{j}^{1}\right) \partial_{u} B_{j, k}^{0}\left(\partial_{z} V^{0}, \partial_{z} V^{0}\right)\right\} .
\end{aligned}
$$

$4^{e}$ SÉRIE - TOME $39-2006-\mathrm{N}^{\circ} 1$ 
For $j \geqslant 1$

$$
\begin{aligned}
F^{j}(t, y, x)= & H\left(U^{0}, d \psi^{0}\right) \partial U^{j}-P^{j-1}(x) \\
G^{j}(t, y, z)= & -\partial_{z}\left(\widetilde{B}_{d, d}^{0} \partial_{z} V^{j+1}\right)+\partial_{z}\left(\widetilde{A}_{d}\left(\mathcal{U}^{0}, d \psi^{0}\right)\left(U^{j+1}+V^{j+1}\right)\right. \\
& \left.-d \widetilde{B}_{d, d}^{0}\left(U^{j+1}+V^{j+1}, \partial_{z} V^{0}\right)+\mathbb{B}\left(\mathcal{U}^{0}\right) d \psi^{j+1}\right)+Q^{j}(t, y, z),
\end{aligned}
$$

where $P^{j}, Q^{j}$ depend only on $\left(\mathcal{U}^{k}, d \psi^{k}\right),\left(\mathcal{U}^{k}, d \psi^{k}, d \psi^{k+1}\right)$ respectively, and their derivatives, for $k \leqslant j$.

Remark 5.3. - (1) Recall that a term like $\left(A_{j}\left(\mathcal{U}^{0}\right)-A_{j}\left(U^{0}\right)\right) \partial_{j} U^{0}$ in (5.14) is evaluated at $(t, y, x, z)=(t, y, 0, z)$. This introduces a fast decaying error which can be incorporated into $G^{1}(t, y, z)$ in view of the fact that $x=\varepsilon \frac{x}{\varepsilon}$. This kind of observation is applied to all such errors.

(2) Define $\mathbb{Q}^{0}(t, y, z)$ for $z \geqslant 0$ by $\int_{+\infty}^{z^{\varepsilon}} Q^{0}(t, y, s) d s$ and for $z \leqslant 0$ by $\int_{-\infty}^{z} Q^{0}(t, y, s) d s$. As we will see shortly, it is essential that the terms involving $\psi^{1}$ do not contribute to the jump of $\mathbb{Q}^{0}$ at $z=0$. These terms come from the last line in (5.14), which can be expressed as

$$
\partial_{z}\left(\sum_{j, k=1}^{d}\left(\nu_{j}^{0} \nu_{k}^{1}+\nu_{k}^{0} \nu_{j}^{1}\right) B_{j, k}^{0} \partial_{z} V^{0}\right) \equiv h(t, y, z) .
$$

Since this derivative is smooth at $z=0$ and $\int_{-\infty}^{+\infty} h(t, y, z) d z=0$, the desired conclusion follows. The same remark applies to the terms involving $\psi^{j+1}$ in the jump of $\mathbb{Q}^{j}$ at $z=0$.

\subsubsection{Boundary profile equations}

In the boundary profile equations $(t, y, x, z)$ is evaluated at $(t, y, 0,0)$. These equations are obtained by substituting the expansions into (5.5) and (5.6) and setting coefficients of the different powers of epsilon equal to 0 . Here $U_{ \pm}^{0}$ or $V_{ \pm}^{0}$ denote limits as $x$ (respectively $z$ ) approaches $0^{ \pm}$.

From (5.5) and (5.6) we obtain the conditions:
(a) $\varepsilon^{0}: U_{+}^{0,1}+V_{+}^{0,1}=U_{-}^{0,1}+V_{-}^{0,1}$,
(b) $\varepsilon^{0}: U_{+}^{0,2}+V_{+}^{0,2}=U_{-}^{0,2}+V_{-}^{0,2}$,
(c) $\varepsilon^{-1}: \partial_{z} V_{+}^{0,2}=\partial_{z} V_{-}^{0,2}$,
(d) $\varepsilon^{0}: \partial_{t} \psi^{0}-\ell(t, y) \cdot \mathcal{U}^{0,2}=\partial_{t} \psi^{0}-\ell(t, y) \cdot \mathcal{U}^{0,2}$,
(a) $\varepsilon^{1}: U_{+}^{1,1}+V_{+}^{1,1}=U_{-}^{1,1}+V_{-}^{1,1}$,
(b) $\varepsilon^{1}: U_{+}^{1,2}+V_{+}^{1,2}=U_{-}^{1,2}+V_{-}^{1,2}$,
(c) $\varepsilon^{0}: \partial_{x} U_{+}^{0,2}+\partial_{z} V_{+}^{1,2}=\partial_{x} U_{-}^{0,2}+\partial_{z} V_{-}^{1,2}$,
(d) $\varepsilon^{1}: \partial_{t} \psi^{1}-\triangle_{y} \psi^{0}+\ell \cdot \mathcal{U}^{1,2}=-\triangle_{y} \psi^{0}$.

and for $j \geqslant 2$,
(a) $\varepsilon^{j}: U_{+}^{j, 1}+V_{+}^{j, 1}=U_{-}^{j, 1}+V_{-}^{j, 1}$,
(b) $\varepsilon^{j}: U_{+}^{j, 2}+V_{+}^{j, 2}=U_{-}^{j, 2}+V_{-}^{j, 2}$,
(c) $\varepsilon^{j-1}: \partial_{x} U_{+}^{j-1,2}+\partial_{z} V_{+}^{j, 2}=\partial_{x} U_{-}^{j-1,2}+\partial_{z} V_{-}^{j, 2}$,
(d) $\varepsilon^{j}: \partial_{t} \psi^{j}-\triangle_{y} \psi^{j-1}+\ell \cdot \mathcal{U}^{j, 2}=0$. 


\subsubsection{Solution of the profile equations}

Notation 5.4. - (1) Set $B_{\nu}:=\widetilde{B}_{d, d}^{0}=\widetilde{B}_{d, d}\left(\mathcal{U}^{0}, \nu^{0}\right)$, an $N \times N$ matrix, and $B_{r}:=B_{\nu}^{22}$, an $s \times s$ matrix (the reduced normal viscosity matrix).

(2) Set $A_{\nu}:=\widetilde{A}_{d}\left(\mathcal{U}^{0}, \nu^{0}\right)$, an $N \times N$ matrix, and $\mathcal{A}=-\left(A_{\nu}^{11}\right)^{-1} A_{\nu}^{12}$, an $(N-s) \times s$ matrix.

We will postpone a careful discussion of regularity of solutions until Proposition 5.7. Here we note simply that we need to assume

$$
U^{0} \in H^{s_{0}}\left(\left[-T_{0}, T_{0}\right] \times \overline{\mathbb{R}}_{ \pm}^{d}\right), \quad \psi^{0} \in H^{s_{0}+1}\left(\left[-T_{0}, T_{0}\right] \times \mathbb{R}^{d}\right)
$$

for some large enough $s_{0}$ depending on $M$.

1. Note that $F^{0}=0$ already by our assumption that $\left(U^{0}, d \psi^{0}\right)$ is a shock.

2. $V_{0}$ and the reduced profile equation. Recall that $G^{-1}=0$ represents equations on $\pm z \geqslant 0$. Define

$$
\mathbb{G}^{-j}(t, y, z)= \begin{cases}\int_{+\infty}^{z} G^{-j}(t, y, s) d s & \text { for } z \geqslant 0, \\ \int_{-\infty}^{z} G^{-j}(t, y, s) d s & \text { for } z \leqslant 0 .\end{cases}
$$

The equations $\mathbb{G}^{-1}=0$ are now

$$
\begin{aligned}
& 0=\tilde{f}_{d}^{1}\left(\mathcal{U}^{0}, d \psi^{0}\right)-\tilde{f}_{d}^{1}\left(U^{0}, d \psi^{0}\right), \\
& B_{r} \partial_{z} \mathcal{U}^{0,2}=\tilde{f}_{d}^{2}\left(\mathcal{U}^{0}, d \psi^{0}\right)-\tilde{f}_{d}^{2}\left(U^{0}, d \psi^{0}\right) .
\end{aligned}
$$

We are given the lead profile and inviscid shock, so we may simply take $V^{0}(t, y, z)=$ $\mathcal{U}^{0}(t, y, 0, z)-U^{0}(t, y, 0)$.

To establish notation used later, we solve for $\mathcal{U}^{0,1}$ in terms of $\mathcal{U}^{0,2}, \mathcal{U}^{0,1}=w\left(\mathcal{U}^{0,2}\right)$, and obtain the reduced profile equation for $\mathcal{U}^{0,2}$

$$
B_{r} \partial_{z} \mathcal{U}^{0,2}=\tilde{f}_{d}^{2}\left(w\left(\mathcal{U}^{0,2}\right), \mathcal{U}^{0,2}, d \psi^{0}\right)-\tilde{f}_{d}^{2}\left(w\left(U^{0,2}\right), U^{0,2}, d \psi^{0}\right),
$$

where $B_{r}$ is the reduced normal viscosity matrix defined above. Observe

$$
\partial_{z} \mathcal{U}^{0,1}=\mathcal{A} \partial_{z} \mathcal{U}^{0,2} ; \quad \partial_{\mathcal{U}^{0,2}} w=\mathcal{A} .
$$

3. Compatibility condition for $V^{1}$. With $\mathbb{Q}^{0}$ as in Remark 5.3 , the equations $\mathbb{G}^{0}=0$ can be written

$$
\begin{aligned}
& \text { (a) } \quad 0=A_{\nu}^{11}\left(U^{1,1}+V^{1,1}\right)+A_{\nu}^{12}\left(U^{1,2}+V^{1,2}\right)+\left(\mathbb{B}\left(\mathcal{U}^{0}\right) d \psi^{1}\right)^{1} \\
& -\left(\widetilde{A}_{d}\left(U^{0}, d \psi^{0}\right) U^{1}+\mathbb{B}\left(U^{0}\right) d \psi^{1}\right)^{1}+\mathbb{Q}^{0,1} . \\
& \text { (b) } B_{\nu}^{22} \partial_{z} V^{1,2}=A_{\nu}^{21}\left(U^{1,1}+V^{1,1}\right)+A_{\nu}^{22}\left(U^{1,2}+V^{1,2}\right)-d B_{\nu}^{22}\left(U^{1}+V^{1}, \partial_{z} V^{0,2}\right) \\
& +\left(\mathbb{B}\left(\mathcal{U}^{0}\right) d \psi^{1}\right)^{2}-\left(\widetilde{A}_{d}\left(U^{0}, d \psi^{0}\right) U^{1}+\mathbb{B}\left(U^{0}\right) d \psi^{1}\right)^{2}+\mathbb{Q}^{0,2} \\
& \equiv \mathbb{D}(t, y, z) \text {. }
\end{aligned}
$$

$\mathcal{U}^{0}$ clearly satisfies the boundary condition (5.16). Suppose for a moment that $\left[\mathcal{U}^{1,2}\right]=$ $\left[U^{1,2}+V^{1,2}\right]=0$. Then (5.23) shows that $\left[\mathcal{U}^{1,1}\right]=0$ if and only if

$$
\left[\widetilde{A}_{d}\left(U^{0}, d \psi^{0}\right) U^{1}+\mathbb{B}\left(U^{0}\right) d \psi^{1}\right]^{1}=\left[\mathbb{Q}^{0,1}\right] .
$$

We seek a condition on $\left[\widetilde{A}_{d}\left(U^{0}, d \psi^{0}\right) U^{1}+\mathbb{B}\left(U^{0}\right) d \psi^{1}\right]^{2}$ that will imply (5.17)(c) assuming that (5.23) and (5.17)(a)(b) hold. Using (5.23)(b) and

$$
\left[\mathcal{U}^{0}\right]=0, \quad\left[\partial_{z} V^{0}\right]=0, \quad\left[U^{1}+V^{1}\right]=0,
$$

$4^{\text {e }}$ SÉRIE - TOME $39-2006-\mathrm{N}^{\circ} 1$ 
we compute

$$
\left[B_{r} \partial_{z} V^{1,2}\right]=[\mathbb{D}]=-\left[\widetilde{A}_{d}\left(U^{0}, d \psi^{0}\right) U^{1}+\mathbb{B}\left(U^{0}\right) d \psi^{1}\right]^{2}+\left[\mathbb{Q}^{0}\right]^{2} .
$$

Thus, (5.17)(c) holds if and only if $\left[B_{r} \partial_{z} V^{1,2}\right]=-\left[B_{r} \partial_{x} U^{0,2}\right]$, that is, if and only if

$$
\left[\widetilde{A}_{d}\left(U^{0}, d \psi^{0}\right) U^{1}+\mathbb{B}\left(U^{0}\right) d \psi^{1}\right]^{2}=\left[\mathbb{Q}^{0}\right]^{2}+\left[B_{r} \partial_{N} U^{0,2}\right]
$$

Eqs. (5.24) and (5.27) give us the boundary conditions for the problem satisfied by $\left(U^{1}, d \psi^{1}\right)$.

For later reference note that we can use (5.23)(a) to express

$$
\begin{aligned}
& U^{1,1}+V^{1,1}=\mathcal{A}\left(U^{1,2}+V^{1,2}\right)+H(t, y, z), \text { where } \\
& H(t, y, z)=-\left(A_{\nu}^{11}\right)^{-1} K(t, y, z) \\
& \quad \text { with } K(t, y, z)=\left(\mathbb{B}\left(\mathcal{U}^{0}\right) d \psi^{1}\right)^{1}-\left(\widetilde{A}_{d}\left(U^{0}, d \psi^{0}\right) U^{1}+\mathbb{B}\left(U^{0}\right) d \psi^{1}\right)^{1}+\mathbb{Q}^{0,1} .
\end{aligned}
$$

4. Solve for $\left(U^{1}, d \psi^{1}\right)$. These are determined by solving

$$
\begin{aligned}
H\left(U^{0}\right) \partial U^{1} & =P^{0}(x), \\
{\left[\mathbb{B}\left(U^{0}\right) d \psi^{1}+A_{N}\left(U^{0}, d \psi^{0}\right) U^{1}\right]^{1} } & =\left[\mathbb{Q}^{0,1}\right], \\
{\left[\mathbb{B}\left(U^{0}\right) d \psi^{1}+A_{N}\left(U^{0}, d \psi^{0}\right) U^{1}\right]^{2} } & =\left[\mathbb{Q}^{0}\right]^{2}+\left[B_{\nu}^{21}\left(A_{\nu}^{11}\right)^{-1} Q^{0,1}\right]+\left[B_{r} \partial_{N} U^{0,2}\right] .
\end{aligned}
$$

The right sides in the boundary and interior equations of (5.30) are initially defined for $t \in$ $\left[-T_{0}, T_{0}\right]$. We can modify them to be zero in $t \leqslant-T_{0}+\delta$, say. We thereby obtain a problem for $\left(U^{1}, d \psi^{1}\right)$ that is forward well-posed in the sense of Majda [26], since $\left(U^{0}, d \psi^{0}\right)$ is uniformly stable and $\psi^{1}$ does not appear on the right side of the boundary equation (Remark 5.3). Thus, we obtain a solution to (5.30) on $\left[-\frac{T_{0}}{2}, T_{0}\right]$.

5. Stable and unstable manifolds. Let $W_{0}^{s}(t, y) \subset \mathbb{R}^{r}$ and $W_{0}^{u}(t, y) \subset \mathbb{R}^{r}$ denote the stable and unstable manifolds of the reduced profile equation (5.21) for the rest points $U_{ \pm}^{0,2}(t, y, 0)$. Our assumptions (Lax shock, Evans condition) imply they intersect transversally in a smooth curve containing $\mathcal{U}^{0,2}(t, y, 0,0)$. With obvious notation let us rewrite (5.21) as

$$
B_{r} \partial_{z} \mathcal{U}^{0,2}=f_{r}^{2}\left(\mathcal{U}^{0,2}, d \psi^{0}\right)-f_{r}^{2}\left(U^{0,2}, d \psi^{0}\right) .
$$

The tangent spaces to $W_{0}^{s}(t, y)$ and $W_{0}^{u}(t, y)$ at $\mathcal{U}^{0,2}(t, y, 0,0)$, denoted $\mathbb{W}_{0}^{s}(t, y)$ and $\mathbb{W}_{0}^{u}(t, y)$, are the stable and unstable subspaces for the equations

$$
B_{r} \partial_{z} V^{1,2}=A_{r}\left(\mathcal{U}^{0,2}, d \psi^{0}\right) V^{1,2}-d_{\mathcal{U}^{0,2}} B_{r}\left(V^{1,2}, \partial_{z} \mathcal{U}^{0,2}\right)
$$

where

$$
A_{r}\left(\mathcal{U}^{0,2}, d \psi^{0}\right)=\partial_{\mathcal{U}^{0,2}} f_{r}=\left(A_{\nu}^{22}-A_{\nu}^{21}\left(A_{\nu}^{11}\right)^{-1} A_{\nu}^{12}\right)\left(w\left(\mathcal{U}^{0,2}\right), \mathcal{U}^{0,2}, d \psi^{0}\right)
$$

and we use $\mathcal{U}^{0,1}=w\left(\mathcal{U}^{0,2}\right)$ to regard $B_{r}$ as a function of $\left(\mathcal{U}^{0,2}, d \psi^{0}\right)$ now.

6. Solve for $V^{1}$. We first obtain $V^{1,2}$ exponentially decaying to 0 as $z \rightarrow \pm \infty$ and then use (5.28) to solve for $V^{1,1}$. It is clear from (5.28) that $\partial_{z} V^{1,1}$ must decay exponentially to 0 , and a closer examination of (5.28) shows the same is true for $V^{1,1}$ itself. In fact, (5.29) implies

$$
H(t, y,+\infty)=U^{1,1}(t, y, 0)-\left(\left.\mathcal{A}\right|_{z=+\infty}\right) U^{1,2} .
$$

The equation for $V^{1,2}$ is (5.23)(b). Because of the compatibility conditions that have been arranged by the choice of $\left(U^{1}, \psi^{1}\right)$, in order to obtain $V^{1}$ satisfying (5.23) and the boundary conditions (5.17)(a),(b),(c), it suffices now to find an exponentially decaying solution to (5.23)(b) such that (5.17)(b) holds: $\left[U^{1,2}+V^{1,2}\right]=0$. 
The solution is based on the observation, which can be verified by direct calculation, that (5.23)(b) has the form

$$
B_{r} \partial_{z} V^{1,2}=A_{r}\left(\mathcal{U}^{0,2}, d \psi^{0}\right) V^{1,2}-d_{\mathcal{U}^{0,2}} B_{r}\left(V^{1,2}, \partial_{z} \mathcal{U}^{0,2}\right)+\mathcal{F}(t, y, z),
$$

where $\mathcal{F}$ is known (expressible in terms of already determined profiles) and exponentially decreasing to 0 as $z \rightarrow \pm \infty$. Now we can solve (5.35) with the correct initial conditions by an argument based on transversality.

Let $W_{1}^{s}(t, y)$ and $W_{1}^{u}(t, y)$ be the linear submanifolds of $\mathbb{R}^{s}$ consisting of initial data at $z=0$ of solutions to (5.35) that decay as $z \rightarrow \pm \infty$. Standard ODE facts [3] imply that $W_{1}^{s}(t, y)$ and $W_{1}^{u}(t, y)$ are translates of $\mathbb{W}_{0}^{s}(t, y)$ and $\mathbb{W}_{0}^{u}(t, y)$. The sum of the dimensions of $W_{1}^{s}(t, y)$ and $W_{1}^{u}(t, y)$ is $s+1$ and they intersect transversally, so their intersection is a line in $\mathbb{R}^{s}$ with direction $\partial_{z} \mathcal{U}^{0,2}(t, y, 0,0)$.

Thus, we should choose initial data

$$
\begin{aligned}
& \left(V_{+}^{1,2}(t, y, 0), V_{-}^{1,2}(t, y, 0)\right) \\
& \quad \in\left(W_{1}^{s}(t, y) \times W_{1}^{u}(t, y)\right) \cap\left\{\left(v_{1}, v_{2}\right) \in \mathbb{R}^{2 s}: v_{1}-v_{2}=U_{-}^{1,2}(t, y, 0)-U_{+}^{1,2}(t, y, 0)\right\} .
\end{aligned}
$$

The above paragraph implies this is a transversal intersection of linear submanifolds of $\mathbb{R}^{2 s}$ of dimensions $s+1$ and $s$ respectively. Call this intersection (which is necessarily nonempty)

$$
\mathcal{L}^{1}(t, y) \text {, the line of connection initial data for } V_{ \pm}^{1,2}(t, y, z) .
$$

For a given $(t, y)$, any point on this line gives a choice of initial data for (5.35) corresponding to a decaying solution that satisfies (5.17)(b). In view of the above discussion we now have $V^{1}$ satisfying (5.23) and (5.17)(a), (b), (c).

To arrange (5.17)(d) as well, note that $\mathcal{L}^{1}(t, y)$ has direction

$$
\mathbb{U}^{0,2}(t, y, 0) \equiv\left(\partial_{z} \mathcal{U}^{0,2}(t, y, 0,0), \partial_{z} \mathcal{U}^{0,2}(t, y, 0,0)\right) .
$$

So

$$
\mathcal{L}^{1}(t, y)=\left\{\mathbb{K}(t, y)+s \mathbb{U}^{0,2}(t, y, 0), s \in \mathbb{R}\right\},
$$

for some initial point $\mathbb{K}(t, y)$. The boundary condition (5.17)(d) holds provided

$$
\partial_{t} \psi^{0}(t, y)+\ell(t, y) \cdot\left(U_{+}^{1,2}(t, y, 0)+V_{+}^{1,2}(t, y, 0)\right)=0 .
$$

Since $\ell(t, y) \cdot \partial_{z} \mathcal{U}^{0,2}(t, y, 0,0) \neq 0$, there is a unique smooth choice of $s(t, y)$ that gives $V_{+}^{1,2}$ satisfying (5.39). We now have exponentially decaying $V_{ \pm}^{1}$ satisfying (5.23) and (5.17).

7. (Continue). The solution of the remaining profile equations follows the same pattern:

$$
\left(U^{1}, \psi^{1}\right) \rightarrow V^{1} \rightarrow\left(U^{2}, \psi^{2}\right) \rightarrow V^{2} \rightarrow \cdots .
$$

The boundary condition for the problem satisfied by $\left(U^{j}, \psi^{j}\right)$ is always the compatibility condition for $V^{j}$. In view of Remark 5.3 the boundary problems for the $\left(U^{j}, \psi^{j}\right)$ are all Majda well-posed, linearized shock problems. The line $\mathcal{L}^{j}(t, y)$ of connection initial data for $V_{ \pm}^{j}$ always has direction $\mathbb{U}^{0,2}(t, y, 0)$.

Provided the index of regularity $s_{0}$ in (5.19) is large enough, this process yields arbitrarily high order approximate solutions with the properties summarized below.

$4^{\text {e }}$ SÉRIE - TOME $39-2006-\mathrm{N}^{\circ} 1$ 


\subsubsection{Summary}

Let $\mathcal{E}(u, \psi)$ be the operator in the left side of (5.2). Our approximate solution $\left(u^{a}, \psi^{a}\right)$ as in (5.8), (5.9) satisfies

$$
\begin{gathered}
\mathcal{E}\left(u^{a}, \psi^{a}\right)=\varepsilon^{M} R^{\varepsilon, M}(t, y, x) \quad \text { on }\left[-\frac{T_{0}}{2}, T_{0}\right] \times \overline{\mathbb{R}}_{ \pm}^{d}, \\
{\left[u^{a}\right]=0 ; \quad\left[\partial_{x} u_{2}^{a}\right]=\varepsilon^{M} r^{M}(t, y) \quad \text { on } x=0,} \\
\partial_{t} \psi^{a}-\varepsilon \triangle_{y} \psi^{a}+\ell(t, y) \cdot u_{2}^{a} \\
=\partial_{t} \psi^{0}-\varepsilon \triangle_{y} \psi^{0}+\ell(t, y) \cdot \mathcal{U}^{0,2}(t, y, 0,0) \quad \text { on } x=0,
\end{gathered}
$$

with remainders $\varepsilon^{M} R^{M}$ and $\varepsilon^{M} r^{M}$ as described in the next step. We can make $\left[\partial_{x} u_{2}^{a}\right]=0$ without changing the other conditions in (5.41) by adding $-x \rho(x) \varepsilon^{M} r^{M}(t, y)$ to $u_{2,+}^{a}$, where $\rho$ is a smooth cutoff equal to one near $x=0$.

Remark 5.5. - (1) The construction does not require the full strength of the uniform stability assumption on the profile $W_{0}(z, p(t, y))$. We need only the properties that follow from this assumption by the Zumbrun-Serre theorem, Theorem A.2, in the low frequency limit; namely, transversality of the connection and uniform stability of the inviscid shock $\left(U^{0}, \psi^{0}\right)$.

(2) Observe that with the extra boundary condition, the higher profiles are uniquely determined by this construction once the leading profile $\mathcal{U}^{0}(t, y, 0, z)$ and inviscid shock $\left(U^{0}(t, y, x), \psi^{0}(t, y)\right)$ are fixed.

In the next proposition we use the following spaces:

DEFINITION 5.6.-

(1) Let $H^{s}$ be the set of functions $U(t, y, x)$ on $\left[-T_{0}, T_{0}\right] \times \mathbb{R}^{d}$ such that the restrictions $U_{ \pm}$ belong to $H^{s}\left(\left[-T_{0}, T_{0}\right] \times \overline{\mathbb{R}}_{ \pm}^{d}\right)$.

(2) Let $\widetilde{H}^{s}$ be the set of functions $V(t, y, z)$ on $\left[-T_{0}, T_{0}\right] \times \mathbb{R}^{d-1} \times \mathbb{R}$ such that the restrictions $V_{ \pm}$belong to $C^{\infty}\left(\overline{\mathbb{R}}_{ \pm}, H^{s}(t, y)\right)$ and satisfy

$$
\left|\partial_{z}^{k} V(t, y, z)\right|_{H^{s}(t, y)} \leqslant C_{k, s} e^{-\delta|z|} \quad \text { for all } k
$$

for some $\delta>0$.

PROPOSITION 5.7 (Approximate solutions). - For given integers $m \geqslant 0$ and $M \geqslant 1$ let

$$
s_{0}>m+\frac{7}{2}+2 M+\frac{d+1}{2} .
$$

Suppose the given inviscid shock $\left(U^{0}, \psi^{0}\right)$ is uniformly stable in the sense of Majda and satisfies $U^{0} \in H^{s_{0}}, U_{ \pm}^{0}(t, y, 0) \in H^{s_{0}}(t, y)$, and $\psi^{0}(t, y) \in H^{s_{0}+1}(t, y)$. Suppose also that the connection given by $W_{0}(z, p(t, y))$ is transversal. Then one can construct $\left(u^{a}, \psi^{a}\right)$ as above,

$$
\begin{gathered}
\psi^{a}=\psi^{0}(t, y)+\varepsilon \psi^{1}(t, y)+\cdots+\varepsilon^{M} \psi^{M}(t, y), \\
u^{a}=\left.\left(\mathcal{U}^{0}(t, y, x, z)+\varepsilon \mathcal{U}^{1}(t, y, x, z)+\cdots+\varepsilon^{M} \mathcal{U}^{M}(t, y, x, z)\right)\right|_{z=\frac{x}{\varepsilon}},
\end{gathered}
$$

where now $U_{+}^{M, 2}(t, y, x)$ is replaced by $U_{+}^{M, 2}(t, y, x)-x \rho(x) r^{M}(t, y)$ for $r^{M}$ as in (5.41). The approximate solution $\left(u^{a}, \psi^{a}\right)$ satisfies

$$
\begin{aligned}
& \mathcal{E}\left(u^{a}, \psi^{a}\right)=\varepsilon^{M} R^{M}(t, y, x) \quad \text { on }\left[-\frac{T_{0}}{2}, T_{0}\right] \times \overline{\mathbb{R}}_{ \pm}^{d}, \\
& {\left[u^{a}\right]=0 ; \quad\left[\partial_{x} u_{2}^{a}\right]=0 \quad \text { on } x=0}
\end{aligned}
$$




$$
\begin{aligned}
& \partial_{t} \psi^{a}-\varepsilon \triangle_{y} \psi^{a}+\ell(t, y) \cdot u_{2}^{a} \\
& \quad=\partial_{t} \psi^{0}-\varepsilon \triangle_{y} \psi^{0}+\ell(t, y) \cdot \mathcal{U}^{0,2}(t, y, 0,0) \quad \text { on } x=0 .
\end{aligned}
$$

We have

$$
\begin{aligned}
& U^{j}(t, y, x) \in H^{s_{0}-2 j}, \quad \psi^{j}(t, y) \in H^{s_{0}-2 j+1}(t, y), \\
& V^{j}(t, y, z) \in \widetilde{H}^{s_{0}-2 j} \\
& r^{M}(t, y) \in H^{s_{0}-2 M-\frac{3}{2}}(t, y)
\end{aligned}
$$

and $R^{M}(t, y, x)$ satisfies

$$
\begin{aligned}
& \text { (a) }\left|\left(\partial_{t}, \partial_{y}, \varepsilon \partial_{x}\right)^{\alpha} R^{M}\right|_{L^{2}(t, y, x)} \leqslant C_{\alpha} \quad \text { for }|\alpha| \leqslant m+\frac{d+1}{2}, \\
& \text { (b) }\left|\left(\partial_{t}, \partial_{y}, \varepsilon \partial_{x}\right)^{\alpha} R^{M}\right|_{L^{\infty}(t, y, x)} \leqslant C_{\alpha} \quad \text { for }|\alpha| \leqslant m .
\end{aligned}
$$

DEFINITION 5.8. - We will refer to $\left(u^{a}, \psi^{a}\right)$ as in Proposition 5.7 as an approximate solution of order $M$.

Proof of Proposition 5.7. - It just remains to check (5.47) and (5.48). $\left(U^{0}, \psi^{0}\right)$ has the given regularity by assumption and $V^{0}$ by construction since $U^{0}{ }_{\mid x=0}$ belongs to $H^{s_{0}}$.

In the linearized shock problem (5.30) satisfied by $\left(U^{1}, \psi^{1}\right)$, the interior forcing term $P^{0}(t, y, x)$ involves terms in which $U^{0}$ is differentiated twice, and so belongs to $H^{s_{0}-2}$. Similarly, the boundary data lies in $H^{s_{0}-2}(t, y)$. Thus, Majda's estimates for (5.30) imply $U^{1} \in H^{s_{0}-2}, U^{1}{ }_{\mid x=0} \in H^{s_{0}-2}$, and $\psi^{1} \in H^{s_{0}-1}$.

$V^{1,2}(t, y, z)$ satisfies an ODE in $z,(5.23)$ (b), in which the coefficients and boundary data at $z=0$ depend on $\left(U^{1}, \psi^{1}\right)$; so $V^{1} \in \widetilde{H}^{s_{0}-2}$. Following this pattern establishes the stated regularity of $\left(U^{j}, \psi^{j}\right)$ and $V^{j}$ for any $j$.

From the boundary profile equation (5.18) we obtain

$$
r^{M}(t, y)=\partial_{x} U_{+}^{M, 2}-\partial_{x} U_{-}^{M, 2} .
$$

Since $U^{M}(t, y, x) \in H^{s_{0}-2 M}$, we have $r^{M} \in H^{s_{0}-2 M-\frac{3}{2}}(t, y)$. This finishes (5.47).

Finally, since $x \rho(x) r^{M}(t, y) \in H^{s_{0}-2 M-\frac{3}{2}}$ and the least regular terms in $R^{M}$ involve two derivatives of $x \rho(x) r^{M}(t, y)$, we obtain (5.48). Observe that we do not deduce (5.48)(b) from (5.48)(a). (5.48)(b) is verified separately using (5.47) and the Sobolev embedding theorem.

Remark 5.9. - (1) Let $m$ and $M$ be given nonnegative integers, and set

$$
\begin{aligned}
u_{0}^{ \pm} & =U_{ \pm}^{0}(t, y, x), \\
u_{\varepsilon}^{\prime \pm} & =\left(U_{ \pm}^{0}(t, y, x)-U_{ \pm}^{0}(t, y, 0)\right)+\varepsilon \mathcal{U}_{ \pm}^{1}\left(t, y, x, \frac{x}{\varepsilon}\right)+\cdots+\varepsilon^{M} \mathcal{U}_{ \pm}^{M}\left(t, y, x, \frac{x}{\varepsilon}\right), \\
h_{\varepsilon}^{\prime} & =\varepsilon d \psi^{1}+\cdots+\varepsilon^{M} d \psi^{M},
\end{aligned}
$$

where the terms on the right in (5.50) are as in Proposition 5.7. It is now easy to check, using (5.47) and the Sobolev embedding theorem, that $u_{0}^{ \pm}, \psi^{0}, u_{\varepsilon}^{\prime \pm}$, and $h_{\varepsilon}^{\prime}$ have the regularity stated in Assumption 4.1 when $m$ in (5.43) is taken to be 0 . These functions are defined by our construction just on the time interval $\left[-T_{0}, T_{0}\right]$, but they can easily be extended to all time with the extensions satisfying Assumption 4.1.

$4^{e}$ SÉRIE - TOME $39-2006-\mathrm{N}^{\circ} 1$ 


\section{Nonlinear stability}

\subsection{Error problem, iteration scheme, and $L^{2}$ estimate}

We seek an exact solution of the transmission problem

$$
\begin{aligned}
& \text { (a) } \mathcal{E}(u, \psi):=\sum_{j=0}^{d-1} A_{j}(u) \partial_{j} u+\widetilde{A}_{d}(u, d \psi) \partial_{d} u-\varepsilon \sum_{j, k=1}^{d} D_{j}\left(B_{j, k}(u) D_{k} u\right)=0, \\
& \text { (b) }[u]=0, \quad\left[\partial_{x} u_{2}\right]=0 \quad \text { on } x=0, \\
& \partial_{t} \psi-\varepsilon \triangle_{y} \psi+\left.\ell(t, y) \cdot u_{2}\right|_{x=0} \\
& =\partial_{t} \psi^{0}-\varepsilon \triangle_{y} \psi^{0}+\ell(t, y) \cdot \mathcal{U}^{0,2}(t, y, 0,0) \quad \text { on } x=0
\end{aligned}
$$

that is close to an approximate solution $\left(u^{a}, \psi^{a}\right)$ of order $M$ as constructed earlier and satisfying (5.46). Here as in (1.10)

$$
D_{j}=\partial_{j}-\left(\partial_{j} \psi\right) \partial_{x} \quad \text { for } 1 \leqslant j<d, \quad D_{d}=\partial_{x} .
$$

We noted in Remark 2.8 that (6.1)(a) is the same as (2.20).

Looking for an exact solution of the form

$$
u=u^{a}+\varepsilon^{L} v, \quad \psi=\psi^{a}+\varepsilon^{L} \phi,
$$

we obtain by subtracting (5.46) from (6.1) the following error problem for $(v, \phi)$ :

$$
\begin{aligned}
& \text { (a) } \varepsilon^{-L}\left(\mathcal{E}\left(u^{a}+\varepsilon^{L} v, \psi^{a}+\varepsilon^{L} \phi\right)-\mathcal{E}\left(u^{a}, \psi^{a}\right)\right)=-\varepsilon^{M-L} R^{M}, \\
& \text { (b) }[v]=0, \quad\left[\partial_{x} v_{2}\right]=0, \quad \partial_{t} \phi-\varepsilon \triangle_{y} \phi+\left.\ell(t, y) \cdot v_{2}\right|_{x=0}=0 \quad \text { on } x=0 .
\end{aligned}
$$

The interior equation (6.4)(a) can be solved, at least formally, by the following iteration scheme, which we shall call scheme I:

$$
\text { (6.5) } \begin{aligned}
\sum_{j=0}^{d-1} & A_{j}\left(u^{a}+\varepsilon^{L} v_{n}\right) \partial_{j} v_{n+1}+\widetilde{A}_{d}\left(u^{a}+\varepsilon^{L} v_{n}, d\left(\psi^{a}+\varepsilon^{L} \phi_{n}\right)\right) \partial_{d} v_{n+1} \\
& +\sum_{j=0}^{d-1}\left(v_{n+1} \cdot \int_{0}^{1} d_{u} A_{j}\left(u^{a}+s \varepsilon^{L} v_{n}\right) d s\right) \partial_{j} u^{a} \\
& +\left(v_{n+1} \cdot \int_{0}^{1} d_{u} \widetilde{A}_{d}\left(u^{a}+s \varepsilon^{L} v_{n}, d\left(\psi^{a}+\varepsilon^{L} \phi_{n}\right)\right) d s\right) \partial_{d} u^{a} \\
& -\varepsilon \sum_{j, k=1}^{d}\left(\partial_{j}-\partial_{j}\left(\psi^{a}+\varepsilon^{L} \phi_{n}\right) \partial_{d}\right)\left(B_{j k}\left(u^{a}+\varepsilon^{L} v_{n}\right)\left(\partial_{k}-\partial_{k}\left(\psi^{a}+\varepsilon^{L} \phi_{n}\right) \partial_{d}\right) v_{n+1}\right) \\
& -\varepsilon \sum_{j, k=1}^{d}\left(\partial_{j}-\partial_{j} \psi^{a} \partial_{d}\right)\left(\left(v_{n+1} \cdot \int_{0}^{1} d_{u} B_{j k}\left(u^{a}+s \varepsilon^{L} v_{n}\right) d s\right)\right. \\
& \left.\times\left(\partial_{k}-\partial_{k}\left(\psi^{a}+\varepsilon^{L} \phi_{n}\right) \partial_{d}\right) u^{a}\right)-\sum_{j=0}^{d-1} \partial_{j} \phi_{n+1} A_{j}\left(u^{a}\right) \partial_{d} u^{a} \\
& +\varepsilon \sum_{j, k=1}^{d} \partial_{j} \phi_{n+1} \partial_{d}\left(B_{j k}\left(u^{a}+\varepsilon^{L} v_{n}\right)\left(\partial_{k}-\partial_{k}\left(\psi^{a}+\varepsilon^{L} \phi_{n}\right) \partial_{d}\right) u^{a}\right)
\end{aligned}
$$




$$
+\varepsilon \sum_{j, k=1}^{d}\left(\partial_{j}-\partial_{j} \psi^{a} \partial_{d}\right)\left(B_{j k}\left(u^{a}\right) \partial_{k} \phi_{n+1} \partial_{d} u^{a}\right)=-\varepsilon^{M-L} R^{M} .
$$

On the boundary we require

$$
\begin{gathered}
{\left[v_{n+1}\right]=0, \quad\left[\partial_{x} v_{n+1}^{2}\right]=0,} \\
\partial_{t} \phi_{n+1}-\varepsilon \triangle_{y} \phi_{n+1}+\left.\ell(t, y) \cdot v_{n+1}^{2}\right|_{x=0}=0 \quad \text { on } x=0 .
\end{gathered}
$$

Remark 6.1. - (1) If all the subscripts $n$ and $n+1$ are removed in (6.5), we have exactly (6.4)(a).

(2) If $\left(v_{n}, \phi_{n}\right)$ is set equal to zero in (6.5), the resulting linear operator appearing on the left in (6.5) and acting on $\left(v_{n+1}, \phi_{n+1}\right)$ is identical to the linearized operator used in the nonlinear stability argument of [12].

(3) Note that many other schemes are possible; for example, one could switch a certain $n$ with $n+1$ in some of the terms of (6.5). It turns out that scheme I leads to a difficulty with higher derivative estimates (see Remark 6.3), so we replace it in the next section by a new scheme.

We can rewrite (6.5)

$$
\begin{aligned}
& \mathcal{L}_{u}\left(u^{a}+\varepsilon^{L} v_{n}, \psi^{a}+\varepsilon^{L} \phi_{n}\right) v_{n+1} \\
& \quad+\mathcal{L}_{\psi}\left(u^{a}+\varepsilon^{L} v_{n}, \psi^{a}+\varepsilon^{L} \phi_{n}\right) \phi_{n+1}=-\varepsilon^{M-L} R^{M},
\end{aligned}
$$

thereby defining $\mathcal{L}_{u}$ and $\mathcal{L}_{\psi}$. The nonlinear error problem (6.4) can now be written

$$
\begin{gathered}
\mathcal{L}_{u}\left(u^{a}+\varepsilon^{L} v, \psi^{a}+\varepsilon^{L} \phi\right) v+\mathcal{L}_{\psi}\left(u^{a}+\varepsilon^{L} v, \psi^{a}+\varepsilon^{L} \phi\right) \phi \\
=-\varepsilon^{M-L} R^{M} \quad \text { on }\left[0, T_{0}\right] \times \mathbb{R}^{d}, \\
{[v]=0, \quad\left[\partial_{x} v_{2}\right]=0, \quad \partial_{t} \phi-\varepsilon \triangle_{y} \phi+\left.\ell(t, y) \cdot v_{2}\right|_{x=0}=0 \quad \text { on } x=0 .}
\end{gathered}
$$

The problem (6.8) needs some initial conditions in order to be well-posed. Choose a $C_{0}^{\infty}$ cutoff function $\theta(t)$ such that

$$
\theta(t)= \begin{cases}1 & \text { for } t \geqslant \frac{-T_{0}}{4} \\ 0 & \text { for } t \leqslant \frac{-T_{0}}{3}\end{cases}
$$

and for an arbitrarily large fixed $K>0$, let $\chi_{K}(x)$ be a $C_{0}^{\infty}$ cutoff such that $\chi_{K}=1$ on $|x| \leqslant K$. We will solve the following forward error problem:

$$
\begin{aligned}
& \text { (a) } \mathcal{L}_{u}\left(u^{a}+\varepsilon^{L} v, \psi^{a}+\varepsilon^{L} \phi\right) v+\chi_{K} \mathcal{L}_{\psi}\left(u^{a}+\varepsilon^{L} v, \psi^{a}+\varepsilon^{L} \phi\right) \phi \\
& =-\varepsilon^{M-L} \theta(t) R^{M} \quad \text { on }\left[-T_{0}, T_{0}\right] \times \mathbb{R}^{d}, \\
& \text { (b) } \quad[v]=0, \quad\left[\partial_{x} v_{2}\right]=0, \quad \partial_{t} \phi-\varepsilon \triangle_{y} \phi+\left.\ell(t, y) \cdot v_{2}\right|_{x=0}=0 \quad \text { on } x=0 \text {, } \\
& \text { (c) } v=0, \quad \phi=0 \quad \text { in } t<\frac{-T_{0}}{3} \text {. }
\end{aligned}
$$

A solution $(v, \phi)$ to (6.10) is then a solution to (6.8) on $\left[0, T_{0}\right] \times \mathbb{R}_{y}^{d-1} \times\{|x| \leqslant K\}$.

Remark 6.2. - The cutoff $\chi_{K}$ is introduced in order to allow us to treat front terms $\phi_{n}$ on an equal footing with interior terms in the Moser estimates below. With these cutoffs we can always assume that $\phi_{n}$ or $\phi_{n+1}$ has been replaced by $\chi(x) \phi_{n}$ or $\chi(x) \phi_{n+1}$, where $\chi(x)$ is any smooth cutoff such that $\chi \chi_{K}=\chi_{K}$. We will usually suppress the $\chi$ attached to fronts in writing the estimates. 


\subsubsection{New iteration scheme: scheme II}

Consider again scheme I (6.5), where now a cutoff $\chi_{K}$ is always inserted as in 6.10(a). We will describe scheme II by prescribing a simple rule for obtaining it from scheme I.

Corresponding to the splitting $\left(v^{1}, v^{2}\right)$, there is a "first equation" and a "second equation". Changes are made only in the second equation of (6.5). Let $\partial_{s}$ denote one of the spatial derivatives $\partial_{x}, \partial_{y}$. Consider the following products, which always occur with matrix coefficients and powers of $\varepsilon^{L}$ attached:

$$
\begin{array}{lll}
\partial_{s} v_{n}^{1} \partial_{s} v_{n+1}^{2}, & \partial_{s} v_{n}^{1} \partial_{s} \phi_{n+1}, & \partial_{s} v_{n}^{1} v_{n+1}, \\
\partial_{s}^{2} \phi_{n} \partial_{s} v_{n+1}^{2}, & \partial_{s}^{2} \phi_{n} \partial_{s} \phi_{n+1}, & \partial_{s}^{2} \phi_{n} v_{n+1} .
\end{array}
$$

The rule is: whenever such a product occurs in (6.5), switch the $n$ and $n+1$; keep the boundary scheme (6.6) as before. This rule defines a new iteration scheme which converges, formally at least, to the problem (6.10)(a)-(b).

Remark 6.3. - (1) When we refer to "products" $\partial_{s} v_{n}^{1} \partial_{s} v_{n+1}^{2}$, for example, we mean, of course, vectors whose entries are products of scalar components of the given factors. Such products appear in the fourth line of (6.5) after the differentiation $\partial_{d}\left(B_{j, k}\left(u^{a}+\varepsilon^{L} v_{n}\right)\right)$ is performed.

(2) The products in (6.11) do not present any difficulty in the proof of the $L^{2}$ estimate for the linearized problem corresponding to scheme I. This is because the size of the terms with subscript $n$ is controlled by Assumption 4.1. The difficulty occurs only in the higher derivative estimates.

Let $\partial=\left(\partial_{t}, \partial_{y}\right)$. To estimate $\partial^{k} v_{n+1}^{1}$, for example, we would apply the $L^{2}$ estimate to the problem obtained by differentiating scheme I $k$ times. A product like $\partial_{s} v_{n}^{1} \partial_{s} v_{n+1}^{2}$ in scheme I gives rise, after $\partial^{k}$ is applied, to a commutator term involving the product

$$
\left(\partial^{k} \partial_{s} v_{n}^{1}\right)\left(\partial_{s} v_{n+1}^{2}\right) \text {. }
$$

Since $k+1$ derivatives fall on $v_{n}^{1}$ and we are trying to estimate $\partial^{k} v_{n+1}^{1}$, such a commutator is an unacceptable forcing term in the induction argument. The $L^{2}$ estimate gives better control over $v_{n}^{2}$ than $v_{n}^{1}$, so the switch resolves this problem. In the case of positive viscosity the control over both components is equally good, so switches are not needed and scheme I is adequate.

We can write the new scheme more explicitly as follows. With $\mathcal{L}_{u}$ and $\mathcal{L}_{\psi}$ as in (6.10) define

$$
\begin{aligned}
\widetilde{\mathcal{L}}_{u} v_{n+1}= & \mathcal{L}_{u}\left(u^{a}+\varepsilon^{L} v_{n}, \psi^{a}+\varepsilon^{L} \phi_{n}\right) v_{n+1} \\
& +\varepsilon^{L} \mathcal{B}_{1} \partial_{s} v_{n+1}^{2}+\varepsilon^{L} \mathcal{B}_{2} v_{n+1}+\varepsilon^{L} \mathcal{A}_{1} \partial_{s} v_{n+1}^{1}, \\
\widetilde{\mathcal{L}}_{\psi} \phi_{n+1}= & \mathcal{L}_{\psi}\left(u^{a}+\varepsilon^{L} v_{n}, \psi^{a}+\varepsilon^{L} \phi_{n}\right) \phi_{n+1}+\varepsilon^{L} \mathcal{B}_{3} \partial_{s} \phi_{n+1}+\varepsilon^{L} \mathcal{A}_{2} \partial_{s}^{2} \phi_{n+1} .
\end{aligned}
$$

Here the matrices $\mathcal{B}_{j}$ are of varying sizes, have vanishing first row, and depend smoothly on their arguments

$$
\mathcal{B}_{j}=\mathcal{B}_{j}\left(u^{a}, d \psi^{a}, \varepsilon \partial_{x} u^{a}, \partial_{y} u^{a}, v_{n}, \partial_{s} \phi_{n}, \partial_{s} v_{n}^{1}, \partial_{s}^{2} \phi_{n}, \varepsilon\right)
$$

for $\varepsilon \in[0,1]$. The matrices $\mathcal{A}_{j}$ also have vanishing first row and depend smoothly on their arguments:

$$
\mathcal{A}_{j}=\mathcal{A}_{j}\left(u^{a}, d \psi^{a}, \varepsilon \partial_{x} u^{a}, \partial_{y} u^{a}, v_{n}, \partial_{s} \phi_{n}, \partial_{s} v_{n}^{2}, \varepsilon\right) .
$$

Remark 6.4. - Each switch precised by the above rule corresponds to making two changes in the original scheme. A bad term of one of the types listed in (6.11) is subtracted, and a corresponding switched term is added. The $\mathcal{B}_{j}$ terms in (6.13) are the subtracted bad terms, and the $\mathcal{A}_{j}$ terms are the added switched terms. 
We will obtain the solution to (6.10) as a limit of solutions $\left(v_{n+1}, \phi_{n+1}\right)$ to problems:

$$
\begin{array}{ll}
\text { (a) } \widetilde{\mathcal{L}}_{u} v_{n+1}+\chi_{K} \widetilde{\mathcal{L}}_{\psi} \phi_{n+1}=-\varepsilon^{M-L} \theta(t) R^{M} & \text { on }\left[-T_{0}, T_{0}\right] \times \mathbb{R}^{d}, \\
\text { (b) }\left[v_{n+1}\right]=0, \quad\left[\partial_{x} v_{n+1}^{2}\right]=0, & \\
& \partial_{t} \phi_{n+1}-\varepsilon \triangle_{y} \phi_{n+1}+\ell(t, y) \cdot v_{n+1}^{2}=0 \quad \text { on } x=0, \\
\text { (c) } v_{n+1}=0, \quad \phi_{n+1}=0 \quad \text { in } t<\frac{-T_{0}}{3}, &
\end{array}
$$

where the coefficients depend on $\left(v_{n}, \phi_{n}\right)$ and other known arguments as described in (6.13)(6.15). We take the first iterate $\left(v_{0}, \phi_{0}\right)=0$.

\subsection{2. $L^{2}$ estimate for scheme II}

It is not yet clear that scheme II (or even the simpler scheme I for that matter) satisfies the $L^{2}$ estimate (4.11) of Theorem 4.2:

$$
\begin{aligned}
& \left\|u_{1}\right\|_{\lambda_{\varepsilon}^{2}}+\left\|u_{2}\right\|_{\lambda_{\varepsilon}^{2} \Lambda_{\varepsilon}}+\sqrt{\varepsilon}\left\|\partial_{x} u_{2}\right\|_{\lambda_{\varepsilon}}+\left|u_{1}(0)\right|_{\lambda_{\varepsilon}} \\
& \quad+\left|u_{2}(0)\right|_{\lambda_{\varepsilon} \Lambda_{\varepsilon}^{1 / 2}}+\varepsilon\left|\partial_{x} u_{2}(0)\right|_{\lambda_{\varepsilon} \Lambda_{\varepsilon}^{-1 / 2}}+|\psi|_{\mu_{\varepsilon} \Lambda_{\varepsilon}} \\
& \leqslant \\
& \leqslant\left(\left\|f_{1}\right\|+\left\|f_{2}\right\|_{\Lambda_{\varepsilon}^{-1}}\right) .
\end{aligned}
$$

To see that it does, we need to relate the interior operator in (6.16) to the operator in the problem (4.2) for which we have proved (6.17).

For $\widetilde{\mathcal{L}}_{u}, \widetilde{\mathcal{L}}_{\psi}$ as in (6.16)(a) with coefficients depending on $\left(u^{a}, \psi^{a}, v_{n}, \phi_{n}, \varepsilon\right)$, define

$$
\begin{aligned}
\widetilde{\mathcal{L}}(u, \psi) & :=\widetilde{\mathcal{L}}_{u} u+\chi_{K} \widetilde{\mathcal{L}}_{\psi} \psi, \\
\widetilde{\mathcal{L}}^{\gamma} & =e^{-\gamma t} \widetilde{\mathcal{L}} e^{\gamma t}
\end{aligned}
$$

and consider the following transmission problem on $\mathbb{R}^{d+1}$ :

$$
\begin{aligned}
& \text { (a) } \widetilde{\mathcal{L}}^{\gamma}(u, \psi)=f, \\
& \text { (b) }[u]=0, \quad\left[\partial_{x} u^{2}\right]=0, \quad\left(\partial_{t}+\gamma\right) \psi-\varepsilon \triangle_{y} \psi+\ell(t, y) \cdot u^{2}=k_{4} \quad \text { on } x=0, \\
& \text { (c) } u=0, \quad \psi=0 \quad \text { in } t<-T_{0} / 3,
\end{aligned}
$$

where we suppose now that $\left(v_{n}, \phi_{n}\right)$ has been extended to $\mathbb{R}^{d+1}$. Observe that when

$$
(u, \psi):=e^{-\gamma t}\left(v_{n+1}, \phi_{n+1}\right), \quad f:=e^{-\gamma t}\left(-\varepsilon^{M-L} \theta(t) R^{M}\right),
$$

then (6.19) is equivalent to (6.16) on $\left[-T_{0}, T_{0}\right] \times \mathbb{R}^{d}$.

Notation 6.5. - (1) Let $D=\left(\partial_{t}, \partial_{y}, \partial_{x}\right), \partial_{s}=\left(\partial_{y}, \partial_{x}\right), \partial=\left(\partial_{t}, \partial_{y}\right)$. Sometimes, we use $D, \partial_{s}$, or $\partial$ to denote just one component of the corresponding vector operator.

(2) Let $\partial^{k}$ denote the collection of operators $\partial_{x_{0}}^{\alpha_{0}} \cdots \partial_{x_{d-1}}^{\alpha_{d-1}}$ such that $\alpha_{0}+\cdots+\alpha_{d-1}=k$. Sometimes $\partial^{k}$ is used to denote a particular member of this collection of operators. We treat $D^{k}$ and $\partial_{s}^{k}$ similarly.

Proposition 6.6. - Fix $M \geqslant L \geqslant 1$ and let $s_{0}$ be the index measuring Sobolev regularity of the inviscid shock (as in Proposition 5.7). Suppose $f \in L^{2}$,

$$
s_{0}>\frac{7}{2}+2 M+\frac{d+1}{2} .
$$

and that there exists $\varepsilon_{0}>0$ such that for $\varepsilon \in\left(0, \varepsilon_{0}\right]$ :

$4^{\text {e }}$ SÉRIE - TOME $39-2006-\mathrm{N}^{\circ} 1$ 


$$
\begin{aligned}
\left\|v_{n}, \phi_{n}\right\|^{* *}:= & \mid v_{n}, D v_{n}, D^{2} v_{n}^{2}, \partial \partial_{x} v_{n}^{1}, \varepsilon \partial_{x}^{2} v_{n}^{1}, \partial_{y}^{2} \partial_{x} v_{n}^{2}, \partial_{x}^{2} \partial_{y} v_{n}^{2}, \\
& \phi_{n}, \partial \phi_{n}, \partial^{2} \phi_{n},\left.\partial^{3} \phi_{n}\right|_{L^{\infty}} \leqslant 1 .
\end{aligned}
$$

There exist positive constants $\gamma_{0}$ and $\varepsilon_{0}$ such that for $\gamma \geqslant \gamma_{0}$ and $0<\varepsilon \leqslant \varepsilon_{0}$, the a priori estimates of Theorems 4.2 and 4.31 for smooth data supported, respectively, near and away from the front, hold for the transmission problem (6.19). In the estimate near the front (6.17), an extra term $C\left|k_{4}\right|_{\lambda_{\varepsilon} \Lambda_{\varepsilon}^{1 / 2}}$ should be included on the right.

Remark 6.7. - (1) With notation as in Proposition 5.7, observe that if we now take

$$
\begin{aligned}
u_{0}^{ \pm}= & U_{ \pm}^{0}(t, y, x) \\
u_{\varepsilon}^{\prime \pm}= & \left(U_{ \pm}^{0}(t, y, x)-U_{ \pm}^{0}(t, y, 0)\right)+\varepsilon \mathcal{U}_{ \pm}^{1}\left(t, y, x, \frac{x}{\varepsilon}\right)+\cdots \\
& +\varepsilon^{M} \mathcal{U}_{ \pm}^{M}\left(t, y, x, \frac{x}{\varepsilon}\right)+\varepsilon^{L} v_{n}^{ \pm}, \\
h_{\varepsilon}^{\prime}= & \varepsilon d \psi^{1}+\cdots+\varepsilon^{M} d \psi^{M}+\varepsilon^{L} d \phi_{n},
\end{aligned}
$$

then Proposition 5.7 and (6.21) imply that $\left(u_{0}, u_{\varepsilon}^{\prime}, h_{\varepsilon}^{\prime}\right)$ satisfies the requirements of Assumption 4.1. For $r_{\varepsilon}$ to be determined (6.47), let us now set

$$
\begin{aligned}
& q^{\varepsilon}(t, y, x)=\left(p(t, y), u_{\varepsilon}^{\prime}(t, y, x, \varepsilon), h_{\varepsilon}^{\prime}(t, y), r_{\varepsilon}(t, y, x)\right) \\
& \quad \text { with } p(t, y)=\left(u_{+}^{0}(t, y, 0), u_{-}^{0}(t, y, 0), d \psi^{0}(t, y)\right) \in \mathcal{C},
\end{aligned}
$$

for $\left(u_{ \pm}^{0}, u_{\varepsilon}^{\prime}, h_{\varepsilon}^{\prime}\right)$ as in (6.23).

(2) We need to allow $k_{4} \neq 0$ in order to carry out the higher derivative estimates below.

Proof of Proposition 6.6. - First we concentrate on the estimate near the front.

1. Principal parts. We shall write $\widetilde{\mathcal{L}}^{\gamma}(u, \psi)$ as the sum of a principal part and a negligible part

$$
\widetilde{\mathcal{L}}^{\gamma}(u, \psi)=\widetilde{\mathcal{L}}_{p}^{\gamma}(u, \psi)+\widetilde{\mathcal{L}}_{n}^{\gamma}(u, \psi),
$$

where $\widetilde{\mathcal{L}}_{n}^{\gamma}(u, \psi)=\left(h_{1}, h_{2}\right)$ is negligible in the sense that $\left\|h_{1}\right\|+\left\|h_{2}\right\|_{\Lambda_{\varepsilon}^{-1}}$ can be absorbed by the left side of the estimate (6.17) by taking $\gamma$ large and $\varepsilon$ small. It then suffices to prove the estimate for the operator $\widetilde{\mathcal{L}}_{p}^{\gamma}$ in place of $\widetilde{\mathcal{L}}^{\gamma}$. We will specify $\widetilde{\mathcal{L}}_{p}^{\gamma}$ by showing how it relates to the operator $\mathcal{L}_{p}^{\gamma}:=e^{-\gamma t} \mathcal{L}_{p} e^{\gamma t}$, where $\mathcal{L}_{p}$ gives the principal part of scheme I (6.5). Writing

$$
\begin{aligned}
& \widetilde{\mathcal{L}}_{p}^{\gamma}(u, \psi)=\widetilde{\mathcal{L}}_{u, p}^{\gamma} u+\chi_{K} \widetilde{\mathcal{L}}_{\psi, p}^{\gamma} \psi, \\
& \mathcal{L}_{p}^{\gamma}(u, \psi)=\mathcal{L}_{u, p}^{\gamma} u+\chi_{K} \mathcal{L}_{\psi, p}^{\gamma} \psi,
\end{aligned}
$$

we will derive explicit expressions for the four operators on the right in (6.26) after providing some notation.

Notation 6.8. - Given $(d+1)$-tuples $\nu^{a}, \nu^{b}$ defined in terms of fronts $\psi^{a}, \psi^{b}$ by $\nu^{a}=$ $\nu\left(d \psi^{a}\right)=\left(-\partial_{t} \psi^{a},-\partial_{1} \psi^{a}, \ldots,-\partial_{d-1} \psi^{a}, 1\right)$, we set:

(1) $\widetilde{B}_{j, k}\left(u, \nu^{a}, \nu^{b}\right)=B_{j, k}(u)$, if $j<d, k<d$.

(2) $\widetilde{B}_{j, d}\left(u, \nu^{a}, \nu^{b}\right)=\sum_{k=1}^{d} B_{j, k}(u) \nu_{k}^{b}$ if $j<d ; \widetilde{B}_{d, k}\left(u, \nu^{a}, \nu^{b}\right)=\sum_{j=1}^{d} B_{j, k}(u) \nu_{j}^{a}$ if $k<d$.

(3) $\widetilde{B}_{d, d}\left(u, \nu^{a}, \nu^{b}\right)=\sum_{j, k=1}^{d} B_{j, k}(u) \nu_{j}^{a} \nu_{k}^{b}$.

(4) $\widetilde{B}_{j, k}\left(u, \nu^{a}\right)=\widetilde{B}_{j, k}\left(u^{a}, \nu^{a}, \nu^{a}\right)$. 
(5) Let $\left(u^{a}, \psi^{a}\right)$ be the approximate solution and set

$$
\begin{aligned}
& u^{b}=u^{a}+\varepsilon^{L} v_{n}, \\
& \psi^{b}=\psi^{a}+\varepsilon^{L} \phi_{n}, \\
& u^{s}=u^{a}+s \varepsilon^{L} v_{n} \quad \text { for } s \in[0,1] .
\end{aligned}
$$

We first rewrite the total operator for scheme I,

$$
\mathcal{L}^{\gamma}(v, \phi)=\mathcal{L}_{u}^{\gamma} v+\chi_{K} \mathcal{L}_{\psi}^{\gamma} \phi
$$

in a form that makes it easier to compare with the operator in (4.2). Here

$$
\mathcal{L}_{u}^{\gamma} v:=\mathbb{A}_{0}^{t}\left(\partial_{t}+\gamma\right) v+\sum_{j=1}^{d} \mathbb{A}_{j}^{t} \partial_{j} v-\varepsilon \sum_{j, k=1}^{d} \widetilde{B}_{j, k}\left(u^{b}, \nu^{b}\right) \partial_{j k}^{2} v+\frac{1}{\varepsilon} E^{t} v,
$$

where, with superscript " $t$ " for total and $\widetilde{A}_{j}(u, \nu):=A_{j}(u)$ for $j<d$,

$$
\begin{aligned}
\mathbb{A}_{0}^{t}= & A_{0}\left(u^{b}\right), \\
\mathbb{A}_{j}^{t} \partial_{j} v= & \widetilde{A}_{j}\left(u^{b}, \nu^{b}\right) \partial_{j} v-\varepsilon \sum_{k=1}^{d} \partial_{k}\left(\widetilde{B}_{k j}\left(u^{b}, \nu^{b}\right)\right) \partial_{j} v \\
& -\varepsilon \sum_{k=1}^{d}\left(\partial_{j} v \cdot \int_{0}^{1} d_{u} \widetilde{B}_{j, k}\left(u^{s}, \nu^{a}, \nu^{b}\right) d s\right) \partial_{k} u^{a}, \quad j=1, \ldots, d, \\
E^{t} v= & \varepsilon \sum_{j=0}^{d}\left(v \cdot \int_{0}^{1} d_{u} \widetilde{A}_{j}\left(u^{s}, \nu^{b}\right) d s\right) \partial_{j} u^{a} \\
& -\varepsilon^{2} \sum_{j, k=1}^{d}\left(v \cdot \int_{0}^{1} d_{u} \widetilde{B}_{j, k}\left(u^{s}, \nu^{a}, \nu^{b}\right) d s\right) \partial_{j k}^{2} u^{a} \\
& -\varepsilon^{2} \sum_{j, k=1}^{d}\left(v \cdot \partial_{j} \int_{0}^{1} d_{u} \widetilde{B}_{j, k}\left(u^{s}, \nu^{a}, \nu^{b}\right) d s\right) \partial_{k} u^{a} .
\end{aligned}
$$

The front term in (6.28) is

$$
\begin{aligned}
\mathcal{L}_{\psi}^{\gamma} \phi= & -\partial_{x} f_{0}\left(u^{a}\right)\left(\partial_{t}+\gamma\right) \phi \\
& -\sum_{j=1}^{d-1}\left(\partial_{x} f_{j}\left(u^{a}\right)-\sum_{k=1}^{d} \varepsilon \partial_{x}\left(\widetilde{B}_{j, k}\left(u^{b}, \nu^{b}\right) \partial_{k} u^{a}\right)\right. \\
& \left.-\sum_{k=1}^{d} \varepsilon \partial_{k}\left(\widetilde{B}_{k, j}\left(u^{a}, \nu^{a}\right) \partial_{x} u^{a}\right)\right) \partial_{j} \phi+\varepsilon \sum_{j, k=1}^{d-1} B_{j, k}\left(u^{a}\right) \partial_{x} u^{a} \partial_{j k}^{2} \phi .
\end{aligned}
$$

To derive the principal parts, we note that

$$
u^{a}(t, y, x)=\left.W(z, \tilde{q}(t, y, x))\right|_{z=\frac{x}{\varepsilon}} \quad \text { for } \tilde{q}_{\varepsilon}(t, y, x)=\left(p(t, y), \tilde{u}_{\varepsilon}^{\prime}, \tilde{h}_{\varepsilon}^{\prime}, 0\right)
$$

with $\tilde{u}_{\varepsilon}^{\prime}, \tilde{h}_{\varepsilon}^{\prime}$ given by (5.50). For $r_{\varepsilon}$ to be determined and $u_{\varepsilon}^{\prime}, h_{\varepsilon}^{\prime}$ as in (6.23), set

$$
q_{\varepsilon}=\left(p(t, y), u_{\varepsilon}^{\prime}, h_{\varepsilon}^{\prime}, r_{\varepsilon}\right)
$$

$4^{\mathrm{e}}$ SÉRIE - TOME $39-2006-\mathrm{N}^{\circ} 1$ 
and, recalling $W(z, q):=W_{0}(z, p)+u^{\prime}$, observe

$$
\begin{aligned}
\partial_{x} u^{a} & =\left.\left(\frac{1}{\varepsilon} \partial_{z} W+\partial_{q} W \cdot \partial_{x} \tilde{q}_{\varepsilon}\right)\right|_{z=\frac{x}{\varepsilon}, q=\tilde{q}_{\varepsilon}} \\
& =\left.\left(\frac{1}{\varepsilon} \partial_{z} W+\partial_{q} W \cdot \partial_{x} \tilde{q}_{\varepsilon}\right)\right|_{z=\frac{x}{\varepsilon}, q=q_{\varepsilon}} .
\end{aligned}
$$

Thus, in the expressions below, it makes no difference whether derivatives of $W$ are evaluated at $\left(\frac{x}{\varepsilon}, \tilde{q}_{\varepsilon}\right)$ or $\left(\frac{x}{\varepsilon}, q_{\varepsilon}\right)$; we choose the latter option.

We also use the fact that differences like

$$
\left(\partial_{j} v \cdot d_{u} \widetilde{B}_{j, d}\left(u^{b}, \nu^{b}\right)\right) \partial_{z} W-\left(\partial_{j} v \cdot \int_{0}^{1} d_{u} \widetilde{B}_{j, d}\left(u^{s}, \nu^{a}, \nu^{b}\right) d s\right) \partial_{z} W, \quad j<d
$$

are negligible. To see this for terms involving $\partial_{j} v^{1}$, use the weight $\Lambda_{\varepsilon}^{-1}$ on the right in (6.17).

We have for the principal part of $\mathcal{L}_{u}^{\gamma}$ :

$$
\mathcal{L}_{u, p}^{\gamma} v=A_{0}\left(u^{b}\right)\left(\partial_{t}+\gamma\right) v+\sum_{j=1}^{d} \mathbb{A}_{j} \partial_{j} v-\varepsilon \sum_{j, k=1}^{d} \widetilde{B}_{j, k}\left(u^{b}, \nu^{b}\right) \partial_{j k}^{2} v+\frac{1}{\varepsilon} E v
$$

where for $1 \leqslant j<d$,

$$
\begin{aligned}
\mathbb{A}_{j} \partial_{j} v= & \widetilde{A}_{j}\left(u^{b}, \nu^{b}\right) \partial_{j} v-\left(\partial_{z} W \cdot d_{u} \widetilde{B}_{d, j}\left(u^{b}, \nu^{b}\right)\right) \partial_{j} v \\
& -\left(\partial_{j} v \cdot d_{u} \widetilde{B}_{j, d}\left(u^{b}, \nu^{b}\right)\right) \partial_{z} W \\
E v= & \left(v \cdot d_{u} \widetilde{A}_{d}\left(u^{b}, \nu^{b}\right)\right) \partial_{z} W-\left(v \cdot d_{u} \widetilde{B}_{d, d}\left(u^{b}, \nu^{b}\right)\right) \partial_{z}^{2} W \\
& -d_{u}^{2} \widetilde{B}_{d, d}\left(u^{b}, \nu^{b}\right)\left(v, \partial_{z} W\right) \partial_{z} W
\end{aligned}
$$

and

$$
\begin{aligned}
\mathbb{A}_{d} \partial_{d} v= & \widetilde{A}_{d}\left(u^{b}, \nu^{b}\right) \partial_{d} v-\left(\partial_{z} W \cdot \partial_{u} \widetilde{B}_{d, d}\left(u^{b}, \nu^{b}\right)\right) \partial_{d} v \\
& -\left(\partial_{d} v \cdot d_{u} \widetilde{B}_{d, d}\left(u^{b}, \nu^{b}\right)\right) \partial_{z} W+\varepsilon \mathcal{C} \partial_{d} v^{1}
\end{aligned}
$$

where

$$
\begin{aligned}
\varepsilon \mathcal{C} \partial_{d} v^{1}= & -\varepsilon\left(\partial_{d} v^{1} \cdot \int_{0}^{1} \partial_{u^{1}} \widetilde{B}_{d, d}\left(u^{s}, \nu^{a}, \nu^{b}\right) d s\right) \partial_{q} W \partial_{x} \tilde{q} \\
& -\varepsilon\left(\partial_{d} v^{1} \cdot \sum_{k=1}^{d-1} \int_{0}^{1} \partial_{u^{1}} \widetilde{B}_{d, k}\left(u^{s}, \nu^{a}, \nu^{b}\right) d s\right) \partial_{k} u^{a} \\
& +\left(\partial_{d} v^{1} \cdot\left(\partial_{u^{1}} \widetilde{B}_{d, d}\left(u^{b}, \nu^{b}\right)-\int_{0}^{1} \partial_{u^{1}} \widetilde{B}_{d, d}\left(u^{s}, \nu^{a}, \nu^{b}\right) d s\right)\right) \partial_{z} W
\end{aligned}
$$

Similarly, the principal part of $\mathcal{L}_{\psi}^{\gamma}$ is

$$
\mathcal{L}_{\psi, p}^{\gamma} \phi=-\frac{1}{\varepsilon} \partial_{z} f_{0}(W)\left(\partial_{t}+\gamma\right) \phi-\frac{1}{\varepsilon} \sum_{j=1}^{d-1} \partial_{z} f_{j}(W) \partial_{j} \phi
$$




$$
\begin{aligned}
& +\frac{1}{\varepsilon} \sum_{j=1}^{d-1} \partial_{z}\left(\left(\widetilde{B}_{j, d}\left(W, \nu^{b}\right)+\widetilde{B}_{d, j}\left(W, \nu^{b}\right)\right) \partial_{z} W\right) \partial_{j} \phi \\
& +\sum_{j, k=1}^{d-1} B_{j, k}(W) \partial_{z} W \partial_{j k}^{2} \phi,
\end{aligned}
$$

where $W(z, q)$ is evaluated at $z=\frac{x}{\varepsilon}, q=q^{\varepsilon}$.

Since

$$
u^{b}=W\left(\frac{x}{\varepsilon}, q_{\varepsilon}\right), \quad \nu^{b}=\nu\left(d \psi^{0}+h_{\varepsilon}^{\prime}\right),
$$

by inspection of (6.36) and (6.40) we may conclude

$$
\begin{aligned}
\mathcal{L}_{u, p}^{\gamma} v+\chi_{K} \mathcal{L}_{\psi, p}^{\gamma} \phi= & \frac{1}{\varepsilon} \mathcal{L}_{0}\left(\frac{x}{\varepsilon}, q^{\varepsilon}, \varepsilon D_{t}, \varepsilon \gamma, \varepsilon D_{y}, \varepsilon \partial_{x}\right) v \\
& -\frac{1}{\varepsilon^{2}} \chi_{K} \mathcal{K}_{0}\left(\frac{x}{\varepsilon}, q^{\varepsilon}, \varepsilon D_{t}, \varepsilon \gamma, \varepsilon D_{y}\right) \phi+\varepsilon \mathcal{C} \partial_{x} v^{1},
\end{aligned}
$$

where $\mathcal{L}_{0}$ and $\mathcal{K}_{0}$ coincide with the operators appearing in (4.2) when the entry $r_{\varepsilon}$ of $q^{\varepsilon}$ is set equal to zero, and

$$
\mathcal{C}=\left(\begin{array}{c}
0 \\
\mathcal{C}^{21}
\end{array}\right)
$$

is given by (6.39).

Returning to (6.13) we see that all the terms involving matrices $\mathcal{A}_{j}$ or $\mathcal{B}_{j}$ there are negligible (in the sense defined below (6.25)) except for $\varepsilon^{L} \mathcal{A}_{1} \partial_{x} v_{n+1}^{1}$. This implies for scheme II:

$$
\begin{aligned}
\widetilde{\mathcal{L}}_{u, p}^{\gamma} v+\chi_{K} \widetilde{\mathcal{L}}_{\psi, p}^{\gamma} \phi= & \frac{1}{\varepsilon} \mathcal{L}_{0}\left(\frac{x}{\varepsilon}, q^{\varepsilon}, \varepsilon D_{t}, \varepsilon \gamma, \varepsilon D_{y}, \varepsilon \partial_{x}\right) v \\
& -\frac{1}{\varepsilon^{2}} \chi_{K} \mathcal{K}_{0}\left(\frac{x}{\varepsilon}, q^{\varepsilon}, \varepsilon D_{t}, \varepsilon \gamma, \varepsilon D_{y}\right) \phi+\varepsilon \mathcal{A} \partial_{x} v^{1},
\end{aligned}
$$

where

$$
\varepsilon \mathcal{A} \partial_{x} v^{1}:=\varepsilon \mathcal{C} \partial_{x} v^{1}+\varepsilon^{L} \mathcal{A}_{1} \partial_{x} v^{1}=\varepsilon\left(\begin{array}{c}
0 \\
\mathcal{A}^{21}
\end{array}\right) \partial_{x} v^{1}
$$

and $\mathcal{A}^{21}$ is a smooth function of its arguments

$$
\mathcal{A}^{21}=\mathcal{A}^{21}\left(u^{a}, d \psi^{a}, \varepsilon \partial_{x} u^{a}, \partial_{y} u^{a}, v_{n}, \partial_{s} \phi_{n}, \partial_{s} v_{n}^{2}, \partial_{x} \tilde{u}_{\varepsilon}^{\prime}, \varepsilon\right) .
$$

2. Definition of $r_{\varepsilon}$. For $\mathcal{A}^{21}$ as in (6.46) set

$$
r_{\varepsilon}(t, y, x)=\varepsilon \mathcal{A}^{21}\left(u^{a}, d \psi^{a}, \varepsilon \partial_{x} u^{a}, \partial_{y} u^{a}, v_{n}, \partial_{s} \phi_{n}, \partial_{s} v_{n}^{2}, \partial_{x} \tilde{u}_{\varepsilon}^{\prime}, \varepsilon\right)
$$

and note that $r_{\varepsilon}$ satisfies the requirements of Assumption 4.1. With this definition of $r_{\varepsilon}$, the transmission problem defined by the operator on the right side of (6.44) together with transmission conditions (6.19) has exactly the form of the problem (4.2) for which the $L^{2}$ estimate of Theorem 4.2 holds. We may allow a nonhomogeneous transmission condition in view of Remark 4.5.

3. Estimate away from the front. A similar but much simpler argument shows that away from the front, the principal part of the operator $\widetilde{\mathcal{L}}^{\gamma}$ in (6.19) is precisely the operator 


$$
\frac{1}{\varepsilon} \mathcal{L}\left(\frac{x}{\varepsilon}, q^{\varepsilon}, \varepsilon D_{t}, \varepsilon \gamma, \varepsilon D_{y}, \varepsilon \partial_{x}\right) u
$$

in (4.159) for which the estimate of Theorem 4.31 was shown to hold.

\subsection{Induction step and higher derivative estimates}

\subsubsection{Preliminaries}

The fractional weights in the estimate (6.17) are not convenient for the nonlinear stability argument, because they lead to difficulties with Moser estimates. So we extract a weaker estimate involving only integral weights on interior norms that is still good enough for the nonlinear stability argument.

Consider again a linear transmission problem like (6.16) on $\Omega:=\left[-T_{0}, T_{0}\right] \times \mathbb{R}^{d}$

$$
\begin{aligned}
& \text { (a) } \widetilde{\mathcal{L}}_{u} u+\chi_{K} \widetilde{\mathcal{L}}_{\psi} \psi=f \quad \text { on } \Omega, \\
& \text { (b) }[u]=0, \quad\left[\partial_{x} u^{2}\right]=0, \quad \partial_{t} \psi-\varepsilon \triangle_{y} \psi+\ell(t, y) \cdot u^{2}=k_{4} \quad \text { on } x=0, \\
& \text { (c) } u=0, \quad \psi_{n+1}=0 \quad \text { in } t<-T_{0} / 3,
\end{aligned}
$$

where now we allow a nonhomogeneous transmission condition.

We continue to use Notation 6.5.

Proposition 6.9. - We make the same hypotheses as in Proposition 6.6, but take all norms on $\Omega$ now. There exist $\gamma_{0}, \varepsilon_{0}$ such that for $\gamma \geqslant \gamma_{0}$ and $\varepsilon \leqslant \varepsilon_{0}$ with $\varepsilon \gamma \leqslant 1$, the problem (6.49) has a unique solution $(u, \psi)$ satisfying:

$$
\begin{aligned}
& \gamma\left\|e^{-\gamma t} u\right\|+\sqrt{\gamma}\left\|e^{-\gamma t}\left(\sqrt{\varepsilon} \partial_{x, y} u_{2}\right)\right\|+\sqrt{\gamma}\left|e^{-\gamma t} u(0)\right|+\sqrt{\gamma}\left|e^{-\gamma t}(\gamma \phi, \partial \phi)\right| \\
& \quad \leqslant C\left(\left\|e^{-\gamma t} f\right\|+\left|e^{-\gamma t}\left(\frac{1}{\sqrt{\varepsilon}} k_{4}, \gamma k_{4}, \partial k_{4}\right)\right|\right) .
\end{aligned}
$$

Proof. - 1. Estimate. The weights in (6.50) are derived directly from those in the estimates (6.17) and (4.158) of Proposition 6.6 after recalling the definitions of $\Lambda_{\varepsilon}$ (4.5), $\lambda_{\varepsilon}$ (4.6), and $\mu_{\varepsilon}$ (4.7). For example, the weight $\mu_{\varepsilon} \Lambda_{\varepsilon}$ on $\psi$ in (6.17) satisfies:

$$
\mu_{\varepsilon} \Lambda_{\varepsilon} \gtrsim \sqrt{\gamma}|\zeta|
$$

This is immediate for $|\varepsilon \zeta| \leqslant 1$. For $|\varepsilon \zeta| \geqslant 1$, we have

$$
\mu_{\varepsilon} \Lambda_{\varepsilon} \sim \frac{\Lambda_{\varepsilon}^{5 / 2}}{\varepsilon^{3 / 2}} \sim \frac{1}{\varepsilon^{3 / 2}}+\frac{|\tau|^{5 / 4}}{\varepsilon^{1 / 4}}+\frac{|\gamma|^{5 / 4}}{\varepsilon^{1 / 4}}+\varepsilon|\eta|^{5 / 2} .
$$

The inequality $a^{2}+b^{2} \geqslant a^{2 / 5} b^{8 / 5}$ implies $\mu_{\varepsilon} \Lambda_{\varepsilon} \gtrsim \sqrt{\gamma}|\tau, \gamma|$, while $a^{2}+b^{2} \geqslant a^{6 / 5} b^{4 / 5}$ implies $\mu_{\varepsilon} \Lambda_{\varepsilon} \gtrsim \sqrt{\gamma}|\eta|$. Similarly,

$$
\left|k_{4}\right|_{\lambda_{\varepsilon} \Lambda_{\varepsilon}^{1 / 2}} \lesssim \frac{1}{\sqrt{\varepsilon}}+|\zeta|^{1 / 2} \lesssim \frac{1}{\sqrt{\varepsilon}}+|\tau, \eta|
$$

for $\gamma \leqslant \frac{1}{\varepsilon}$.

2. Existence, uniqueness, causality. A standard density argument [30, Section 5.1] using the a priori estimate (6.50) gives uniqueness. For existence, observe that for a fixed $\varepsilon$ the (nonstandard) linear transmission problem (6.19) can be solved by the following scheme:

$$
\begin{gathered}
\widetilde{\mathcal{L}}_{u, p}^{\gamma} u_{n+1}=f-\chi_{K} \widetilde{\mathcal{L}}_{\psi, p}^{\gamma} \psi_{n}, \\
{\left[u_{n+1}\right]=0, \quad\left[\partial_{x} u_{n+1}^{2}\right]=0 \quad \text { on } x=0,}
\end{gathered}
$$




$$
\begin{gathered}
u_{n+1}=0 \quad \text { in } t<-T_{0} / 3, \\
\partial_{t} \psi_{n+1}-\varepsilon \triangle_{y} \psi_{n+1}=-\ell \cdot u_{n}^{2}+k_{4} \quad \text { on } x=0, \\
\psi_{n+1}=0 \quad \text { in } t<-T_{0} / 3,
\end{gathered}
$$

with $\left(u_{0}, \psi_{0}\right)=0$. We then apply the uniform estimates (6.50) to the solutions of (6.19) so obtained.

A classical argument [30, Lemma 5.2] using the estimate (6.50) shows that causality holds: if the data $f$ and $k_{4}$ vanish in $t<T_{0}$ in (6.19), then so does $(u, \psi)$. This allows us to restrict the norms in (6.50) to $\Omega$.

DEFINITION 6.10. - (1) Let $\mu=\frac{\gamma}{\varepsilon}$. On $\Omega$ set

$$
\begin{gathered}
|u|_{p, \mu, \gamma}=\sum_{j=0}^{p} \mu^{p-j}\left|e^{-\gamma t} \partial^{j} u\right|_{L^{2}(\Omega)}, \\
|u(0)|_{p, \mu, \gamma}=\sum_{j=0}^{p} \mu^{p-j}\left|e^{-\gamma t} \partial^{j} u(0)\right|_{L^{2}(t, y)},
\end{gathered}
$$

where we always suppose $0<\varepsilon \leqslant 1 \leqslant \gamma$.

(2) Set $|u|_{*}=|u|_{L^{\infty}(\Omega)}$,

$$
\begin{aligned}
\left\|v_{n}, \phi_{n}\right\|^{* * *}:= & \mid v_{n}, D v_{n}, D^{2} v_{n}^{2}, \partial \partial_{x} v_{n}^{1}, \varepsilon \partial_{x}^{2} v_{n}^{1}, \partial_{y}^{2} \partial_{x} v_{n}^{2}, \partial_{x}^{2} \partial_{y} v_{n}^{2}, \phi_{n}, \partial \phi_{n}, \\
& \partial^{2} \phi_{n},\left.\partial^{3} \phi_{n}\right|_{*},
\end{aligned}
$$

and define $\left\|v_{n}, \phi_{n}\right\|^{* *}$ by the right side of (6.57) with $\varepsilon \partial_{x}^{2} v_{n}^{1}$ removed.

Observe that

$$
|u|_{p-1, \mu, \gamma} \leqslant \frac{1}{\mu}|u|_{p, \mu, \gamma} \leqslant \frac{\varepsilon}{\gamma}|u|_{p, \mu, \gamma} .
$$

The following version of the standard Sobolev estimate is easily proved using the Fourier transform after taking extensions from $\Omega_{ \pm}$to $\mathbb{R}_{ \pm}^{d+1}$ and observing that $e^{-\gamma t} \sim C(\gamma)$ on $\left[-T_{0}, T_{0}\right]$.

Proposition 6.11 (Sobolev estimate). - Let $0<\delta<p-\frac{d}{2}$. Then on $\Omega$ we have:

$$
|u|_{*} \leqslant \mu^{-\delta} C_{1}(\gamma)\left(|u|_{p, \mu \gamma}+\left|\partial_{x} u\right|_{p, \mu, \gamma}\right) .
$$

DEFINITION 6.12 (Norms for iteration). - For nonnegative integers $k$ define

$$
\begin{aligned}
\|v, \phi\|_{k, \mu, \gamma}= & \left|v, \sqrt{\varepsilon} \nabla_{x, y} v^{2}\right|_{k, \mu, \gamma}+\left|\partial_{x} v^{1}\right|_{k-1, \mu, \gamma}+\left|\partial_{x}^{2} v^{1}\right|_{k-2, \mu, \gamma} \\
& +\left|\partial_{x}^{2} v^{2}\right|_{k-2, \mu, \gamma}+\left|\partial_{x}^{3} v^{2}\right|_{k-3, \mu, \gamma}+|v(0), \phi, d \phi|_{k, \mu, \gamma}
\end{aligned}
$$

where a norm $|u|_{j, \mu, \gamma}$ is defined to be zero if $j<0$.

\subsubsection{Induction step}

The following proposition, proved below, is the main higher derivative estimate.

PROPOSITION 6.13. - Consider the linear transmission problem defining scheme II (6.16). Assume $L \geqslant 2, k \geqslant 0, M-L-k \geqslant 0$, and suppose that $s_{0}$, the index measuring Sobolev regularity of the inviscid shock as in Proposition 5.7, satisfies

$$
s_{0}>k+\frac{7}{2}+2 M+\frac{d+1}{2} .
$$

$4^{e}$ SÉRIE - TOME $39-2006-\mathrm{N}^{\circ} 1$ 
Suppose also that

$$
\left\|v_{n}, \phi_{n}\right\|^{* * *} \leqslant 1
$$

For $\gamma$ fixed large enough there exists $\varepsilon_{0}(\gamma)$ such that for $0<\varepsilon \leqslant \varepsilon_{0}(\gamma)$, the unique solution $\left(v_{n+1}, \phi_{n+1}\right)$ of (6.16) satisfies

$$
\left\|v_{n+1}, \phi_{n+1}\right\|_{k, \mu, \gamma} \leqslant C(k)\left(\frac{1}{\sqrt{\gamma}}+\left\|v_{n+1}, \phi_{n+1}\right\|^{* *}\right)\left\|v_{n}, \phi_{n}\right\|_{k, \mu, \gamma}+C(\gamma) \varepsilon^{M-L-k}
$$

Assuming the above proposition for the moment, we now prove

Proposition 6.14 (Induction step). - Assume $L \geqslant 2$,

$$
\frac{d}{2}+4<k<M-L
$$

and $s_{0}$ satisfies (6.61). For $C(\gamma)$ as in (6.63) assume

$$
\begin{aligned}
& \text { (a) }\left\|v_{n}, \phi_{n}\right\|^{* * *} \leqslant 1 \\
& \text { (b) }\left\|v_{n}, \phi_{n}\right\|_{k, \mu, \gamma} \leqslant 2 C(\gamma) \varepsilon^{M-L-k}
\end{aligned}
$$

for $\gamma$ large enough and $\varepsilon(\gamma)$ sufficiently small. There exists $\gamma_{0}$ such that for fixed $\gamma \geqslant \gamma_{0}$ and $0<\varepsilon \leqslant \varepsilon_{0}(\gamma)$ small enough, the same estimates hold for $\left(v_{n+1}, \phi_{n+1}\right)$. The choices of $\gamma_{0}$ and $\varepsilon_{0}(\gamma)$ are independent of $n$.

Proof. - Recall $\mu=\frac{\gamma}{\varepsilon}$ and note that by the Sobolev estimate

$$
\|v, \phi\|^{* *} \leqslant C_{1}(\gamma) \mu^{-\delta}\|v, \phi\|_{k, \mu, \gamma},
$$

where $0<\delta<k-4-\frac{d}{2}$.

Let $a_{n}=\left\|v_{n}, \phi_{n}\right\|_{k, \mu, \gamma} \leqslant 2 C(\gamma) \varepsilon^{M-L-k}$. The estimates (6.63) and (6.66) imply

$$
a_{n+1} \leqslant C(k)\left(\frac{1}{\sqrt{\gamma}}+C_{1}(\gamma) \mu^{-\delta} a_{n+1}\right) 2 C(\gamma) \varepsilon^{M-L-k}+\varepsilon^{M-L-k} C(\gamma) .
$$

Choose $\gamma_{0}$ and $\varepsilon_{0}(\gamma)$ such that for $\gamma \geqslant \gamma_{0}$ and $0<\varepsilon \leqslant \varepsilon_{0}(\gamma)$ we have

$$
\begin{aligned}
& 2 C(k) \frac{1}{\sqrt{\gamma}} \leqslant \frac{1}{3}, \\
& 2 C(k) C_{1}(\gamma) \mu^{-\delta} C(\gamma) \varepsilon^{M-L-k} \leqslant \frac{1}{3},
\end{aligned}
$$

Then

$$
a_{n+1} \leqslant 2 C(\gamma) \varepsilon^{M-L-k} .
$$

Shrinking $\varepsilon_{0}(\gamma)$ if necessary and using (6.66), (6.69) we have $\left\|v_{n+1}, \phi_{n+1}\right\|^{* *} \leqslant 1$. Solving for $\varepsilon \partial_{x}^{2} v_{n+1}^{1}$ using the first component of Eq. (6.16)(a) and shrinking $\varepsilon_{0}(\gamma)$ once more, we obtain $\left\|v_{n+1}, \phi_{n+1}\right\|^{* * *} \leqslant 1$.

Proof of Proposition 6.13. -

1. Preliminaries. Observe that the case $k=0$ follows immediately from Proposition 6.9.

The main extra tool we need for the higher derivative estimates is the following weighted version of the standard Moser estimate [9, Lemma 2.1.2]. 
Lemma 6.15 (Moser estimates). - For $k \in \mathbb{N}=\{0,1,2, \ldots\}$ let $\alpha_{1}+\cdots+\alpha_{r} \leqslant j \leqslant k$, $\alpha_{i} \in \mathbb{N}$. Then

$$
\mu^{k-j}\left|\left(\partial^{\alpha_{1}} w_{1}\right) \cdots\left(\partial^{\alpha_{r}} w_{r}\right)\right|_{0, \mu, \gamma} \leqslant C \sum_{i=1}^{r}\left|w_{i}\right|_{k, \mu, \gamma}\left(\prod_{j \neq i}\left|w_{j}\right|_{*}\right) .
$$

2. Tangential higher derivative estimates. The first step in proving (6.63) is to show

$$
\begin{aligned}
\left\|v_{n+1}, \phi_{n+1}\right\|_{k, \mu, \gamma}^{\prime} \leqslant & C\left(\frac{1}{\sqrt{\gamma}}+\left\|v_{n+1}, \phi_{n+1}\right\|^{* *}\right)\left\|v_{n}, \phi_{n}\right\|_{k, \mu, \gamma} \\
& +\frac{C}{\sqrt{\gamma}}\left\|v_{n+1}, \phi_{n+1}\right\|_{k, \mu, \gamma}+C(\gamma) \varepsilon^{M-L-k},
\end{aligned}
$$

where

$$
\|v, \phi\|_{k, \mu, \gamma}^{\prime}=\left|v, \sqrt{\varepsilon} \nabla_{x, y} v^{2}\right|_{k, \mu, \gamma}+|v(0), \phi, \partial \phi|_{k, \mu, \gamma} .
$$

To estimate $\left\|v_{n+1}, \phi_{n+1}\right\|_{k, \mu, \gamma}^{\prime}$ we apply the $L^{2}$ estimate (6.9) to the problem satisfied by $\mu^{k-j} \partial^{j}\left(v_{n+1}, \phi_{n+1}\right)$. Commuting $\mu^{k-j} \partial^{j}$ through (6.16)(a), we obtain forcing that is a sum of

$$
-\varepsilon^{M-L} \mu^{k-j} \partial^{j}\left(\theta(t) R^{M}\right)
$$

and commutator terms. In addition there is a nonzero commutator coming from the third transmission condition in (6.16)(b); this is why we allow $k_{4} \neq 0$ in (6.49).

Thus, the $L^{2}$ estimate gives

$$
\begin{aligned}
\mu^{k-j} & \left|\partial^{j} v_{n+1}, \sqrt{\varepsilon} \nabla_{x, y} \partial^{j} v_{n+1}^{2}\right|_{0, \mu, \gamma}+\mu^{k-j}\left|\partial^{j} v(0), \partial^{j} \phi, \partial^{j} \partial \phi\right|_{0, \mu, \gamma} \\
\leqslant & \frac{C}{\sqrt{\gamma}}\left(\mu^{k-j}\left|\varepsilon^{M-L} \partial^{j}\left(\theta(t) R^{M}\right)\right|_{0, \mu, \gamma}+\mu^{k-j} \mid \text { interior commutators }\left.\right|_{0, \mu, \gamma}\right) \\
& \quad+\frac{C}{\sqrt{\gamma}}\left(\mu^{k-j}\left|\frac{1}{\sqrt{\varepsilon}}\left[\partial^{j}, \ell\right] v_{n+1}^{2}(0), \gamma\left[\partial^{j}, \ell\right] v_{n+1}^{2}(0), \partial\left[\partial^{j}, \ell\right] v_{n+1}^{2}(0)\right|_{0, \mu, \gamma}\right) .
\end{aligned}
$$

We treat the interior commutators below. The $R^{M}$ term on the right in (6.74) is $\lesssim C(\gamma) \varepsilon^{M-L-k}$ and the last term is $\lesssim \frac{1}{\sqrt{\gamma}}|v(0)|_{k, \mu, \gamma}$; both estimates are compatible with (6.71).

NOTATION 6.16. - (1) Let $w_{n}=\left(v_{n}, \sqrt{\varepsilon} \partial_{x, y} v_{n}^{2}, \phi_{n}, d \phi_{n}\right)$.

(2) Let $U^{a, \varepsilon}=\left(u^{a}, d \psi^{a}, \partial_{y}\left(u^{a}, d \psi^{a}\right), \varepsilon \partial_{x} u^{a}, \sqrt{\varepsilon}\right)$ and let $\mathbb{A}$ denote a smooth function

$$
\mathbb{A}=\mathbb{A}\left(U^{a, \varepsilon}, \varepsilon^{L-1} w_{n}\right) .
$$

Denote by $\mathbb{B}_{n}$ a smooth function

$$
\mathbb{B}=\mathbb{B}\left(U^{a, \varepsilon}, \varepsilon \partial_{s}^{2} u^{a}, \partial_{x} u^{a}, \varepsilon^{L-1} w_{n}\right),
$$

where the second and third arguments only occur linearly in terms where they appear. $\mathbb{A}$ and $\mathbb{B}$ may change from term to term.

(3) For $s \in\{1,2,3, \ldots\}$ and a function $u$ with components $u_{i}$, denote by $\partial^{\langle s\rangle} u$ the set of products of the form $\left(\partial^{s_{1}} u_{i_{1}}\right) \ldots\left(\partial^{s_{r}} u_{i_{r}}\right)$ where $s_{1}+\cdots+s_{r}=s, s_{i} \geqslant 1$. If $s=0$, set $\partial^{\langle 0\rangle} u=1$.

For the purpose of tangential higher derivative estimates we can write (6.16)(a) in the much simpler form

$$
\mathbb{A} D w_{n+1}+\mathbb{B} w_{n+1}=-\varepsilon^{M-L} \theta(t) R^{M} .
$$

$4^{e}$ SÉRIE - TOME $39-2006-\mathrm{N}^{\circ} 1$ 
This is because the $L^{2}$ estimate gives the same interior control over all components of $w_{n+1}$ (recall Remark 6.2).

Consider the commutator term

$$
\mu^{k-j}\left|\left[\mathbb{A} D, \partial^{j}\right] w_{n+1}\right|_{0, \mu, \gamma}
$$

This leads to a sum of terms of the form

$$
\begin{aligned}
& \mu^{k-j}\left|\mathbb{A} \partial^{\langle r\rangle}\left(U^{a, \varepsilon}\right) \partial^{\langle s\rangle}\left(\varepsilon^{L-1} w_{n}\right) \partial^{t} D w_{n+1}\right|_{0, \mu, \gamma} \\
& \leqslant \mu^{k-j} C\left|\partial^{\langle s\rangle}\left(\varepsilon^{L-1} w_{n}\right) \partial^{t} D w_{n+1}\right|_{0, \mu, \gamma},
\end{aligned}
$$

where $r+s+t=j$ and $t<j$.

If $s \neq 0$ we estimate

$$
\mu^{k-j}\left|\partial^{(s-1)} \partial\left(\varepsilon^{L-1} w_{n}\right) \partial^{t} D w_{n+1}\right|_{0, \mu, \gamma}
$$

with $(s-1)+t \leqslant j-1$. By Moser this is $\leqslant$

$$
\begin{aligned}
& C\left(\left|\partial\left(\varepsilon^{L-1} w_{n}\right)\right|_{k-1, \mu, \gamma}\left|D w_{n+1}\right|_{*}+\left|D w_{n+1}\right|_{k-1, \mu, \gamma}\left|\partial\left(\varepsilon^{L-1} w_{n}\right)\right|_{*}\right) \\
& \quad \leqslant C\left(\left\|\left(v_{n}, \phi_{n}\right)\right\|_{k, \mu, \gamma}\left|v_{n+1}, \phi_{n+1}\right|^{* *}+\left\|v_{n+1}, \phi_{n+1}\right\|_{k, \mu, \gamma}\right) .
\end{aligned}
$$

If $s=0$ we obtain immediately $(6.79) \leqslant$

$$
C\left|D w_{n+1}\right|_{k-1, \mu, \gamma} \leqslant C\left\|v_{n+1}, \phi_{n+1}\right\|_{k, \mu, \gamma} .
$$

After dividing by $\sqrt{\gamma}$ as allowed by (6.74) we see that the commutator (6.78) gives terms on the right compatible with the intermediate estimate (6.71).

Next consider

$$
\mu^{k-j}\left|\left[\mathbb{B}, \partial^{j}\right] w_{n+1}\right|_{0, \mu, \gamma},
$$

which yields terms

$$
\mu^{k-j}\left|\partial^{\langle r\rangle}\left(\varepsilon \partial_{s}^{2} u^{a}, \partial_{x} u^{a}\right) \partial^{\langle s\rangle}\left(\varepsilon^{L-1} w_{n}\right) \partial^{t} w_{n+1}\right|_{0, \mu, \gamma},
$$

where $r+s+t \leqslant j, t \leqslant j-1$.

If $s \neq 0,(6.84)$ is $\leqslant$

$$
\begin{array}{rl}
\mu^{k-j} & C\left|\partial^{\langle r\rangle}\left(\varepsilon \partial_{s}^{2} u^{a}, \partial_{x} u^{a}\right) \partial^{(s-1)} \partial\left(\varepsilon^{L-1} w_{n}\right) \partial^{t} w_{n+1}\right|_{0, \mu, \gamma} \\
\leqslant & C\left|\left(\varepsilon^{2} \partial_{s}^{2} u^{a}, \varepsilon \partial_{x} u^{a}\right)\right|_{*}\left(\left|\partial\left(\varepsilon^{L-2} w_{n}\right)\right|_{k-1, \mu, \gamma}\left|w_{n+1}\right|_{*}\right. \\
\left.\quad+\left|\partial\left(\varepsilon^{L-2} w_{n}\right)\right|_{*}\left|w_{n+1}\right|_{k-1, \mu, \gamma}\right) .
\end{array}
$$

If $s=0,(6.84)$ is $\leqslant$

$$
\begin{aligned}
\mu^{k-j} C\left|\partial^{\langle r\rangle}\left(\varepsilon \partial_{s}^{2} u^{a}, \partial_{x} u^{a}\right) \partial^{t} w_{n+1}\right|_{0, \mu, \gamma} & \leqslant C\left|\partial^{\langle r\rangle}\left(\varepsilon \partial_{s}^{2} u^{a}, \partial_{x} u^{a}\right)\right|_{*}\left|w_{n+1}\right|_{k-1, \mu, \gamma} \\
& \leqslant \frac{C}{\gamma}\left|\partial^{\langle r\rangle}\left(\varepsilon^{2} \partial_{s}^{2} u^{a}, \varepsilon \partial_{x} u^{a}\right)\right|_{*}\left|w_{n+1}\right|_{k, \mu, \gamma} .
\end{aligned}
$$

In both cases the estimate is compatible with the intermediate estimate (6.71).

3. Normal derivative estimates. For the normal derivative estimates it is more convenient to write (6.16)(a) in the form

$$
\begin{aligned}
& \text { (a) } \partial_{x} v_{n+1}^{1}+\mathbb{A} \widetilde{D} w_{n+1}+\mathbb{B} w_{n+1}=\varepsilon^{M-L} \mathbb{A} R_{1}^{M}, \\
& \text { (b) } \varepsilon \partial_{x}^{2} v_{n+1}^{2}+\mathbb{A} D^{*} w_{n+1}+\mathbb{B} w_{n+1}=\varepsilon^{M-L} \mathbb{A} R_{2}^{M},
\end{aligned}
$$


where

$$
\begin{aligned}
\widetilde{D} w_{n+1} & =\left(\partial v_{n+1}^{1}, D v_{n+1}^{2}, D \phi_{n+1}\right), \\
D^{*} w_{n+1} & =\left(D v_{n+1}, \varepsilon \partial_{y} \partial_{x, y} v_{n+1}^{2}, D \phi_{n+1}, \partial_{s}^{2} \phi_{n+1}\right) .
\end{aligned}
$$

One first shows directly from (6.87)(a) that

$$
\left|\partial_{x} v_{n+1}^{1}\right|_{k-1, \mu, \gamma} \leqslant C\left(\left\|v_{n+1}, \phi_{n+1}\right\|_{k, \mu, \gamma}^{\prime}+(\operatorname{RHS} \text { of }(6.71))\right):=\mathcal{R} .
$$

Note that we already have

$$
\left|\partial_{x} v_{n+1}^{2}\right|_{k-1, \mu, \gamma} \leqslant \frac{1}{\mu}\left|\partial_{x} v_{n+1}^{2}\right|_{k, \mu, \gamma} \leqslant \frac{\sqrt{\varepsilon}}{\gamma}\left\|v_{n+1}, \phi_{n+1}\right\|_{k, \mu, \gamma}^{\prime} .
$$

Next show using (6.87)(b) that

$$
\left|\partial_{x}^{2} v_{n+1}^{2}\right|_{k-2, \mu, \gamma} \leqslant C\left(\mathcal{R}+\left|\partial_{x} v_{n+1}^{1}\right|_{k-1, \mu, \gamma}\right) .
$$

For example, let us check the term

$$
\frac{1}{\varepsilon}\left|\mathbb{A} D^{*} w_{n+1}\right|_{k-2, \mu, \gamma} \leqslant \frac{1}{\gamma}\left|\mathbb{A} D^{*} w_{n+1}\right|_{k-1, \mu, \gamma} .
$$

This leads to terms like

$$
\frac{1}{\gamma} \mu^{k-1-j}\left|\partial^{\langle s\rangle}\left(\varepsilon^{L-1} w_{n}\right) \partial^{t} D^{*} w_{n+1}\right|_{0, \mu, \gamma}
$$

with $s+t \leqslant j \leqslant k-1$. If $s=0(6.93)$ is $\leqslant$

$$
\frac{1}{\gamma}\left|D^{*} w_{n+1}\right|_{k-1, \mu, \gamma}
$$

If $s \neq 0(6.93)$ is

$$
\begin{aligned}
& \frac{1}{\gamma} \mu^{k-1-j}\left|\partial^{(s-1)} \partial\left(\varepsilon^{L-1} w_{n}\right) \partial^{t} D^{*} w_{n+1}\right|_{0, \mu, \gamma} \\
& \quad \leqslant \frac{1}{\gamma}\left(\left|\partial\left(\varepsilon^{L-1} w_{n}\right)\right|_{k-2, \mu, \gamma}\left|D^{*} w_{n+1}\right|_{*}+\left|\partial\left(\varepsilon^{L-1} w_{n}\right)\right|_{*}\left|D^{*} w_{n+1}\right|_{k-2, \mu, \gamma}\right) .
\end{aligned}
$$

In both cases the estimate is compatible with (6.91).

Next we differentiate $(6.87)$ (a) to show

$$
\left|\partial_{x}^{2} v_{n+1}^{1}\right|_{k-2, \mu, \gamma} \leqslant C\left(\mathcal{R}+\left|\partial_{x} v_{n+1}^{1}\right|_{k-1, \mu, \gamma}+\left|\partial_{x}^{2} v_{n+1}^{2}\right|_{k-2, \mu, \gamma}\right) .
$$

For example consider the term

$$
\left|\mathbb{A} \partial_{x} \partial v_{n+1}^{1}\right|_{k-2, \mu, \gamma}
$$

This leads to terms like

$$
\mu^{k-2-j}\left|\partial^{\langle s\rangle}\left(\varepsilon^{L-1} w_{n}\right) \partial^{t} \partial_{x} \partial v_{n+1}^{1}\right|_{0, \mu, \gamma},
$$

with $s+t \leqslant j \leqslant k-2$. If $s=0$ (6.98) is $\leqslant$

$$
C\left|\partial_{x} \partial v_{n+1}^{1}\right|_{k-2, \mu, \gamma} .
$$

If $s \neq 0$ (6.98) equals

$4^{\mathrm{e}}$ SÉRIE - TOME $39-2006-\mathrm{N}^{\circ} 1$ 
(6.100)

$$
\begin{aligned}
& \mu^{k-2-j}\left|\partial^{(s-1)} \partial\left(\varepsilon^{L-1} w_{n}\right) \partial^{t} \partial_{x} \partial v_{n+1}^{1}\right|_{0, \mu, \gamma} \\
& \quad \leqslant\left(\left|\partial\left(\varepsilon^{L-1} w_{n}\right)\right|_{k-3, \mu, \gamma}\left|\partial_{x} \partial v_{n+1}^{1}\right|_{*}+\left|\partial\left(\varepsilon^{L-1} w_{n}\right)\right|_{*}\left|\partial_{x} \partial v_{n+1}^{1}\right|_{k-3, \mu, \gamma}\right) .
\end{aligned}
$$

In both cases the estimate is compatible with (6.91).

Finally, we differentiate (6.87)(b) to show

$$
\begin{aligned}
& \left|\partial_{x}^{3} v_{n+1}^{2}\right|_{k-3, \mu, \gamma} \\
& \quad \leqslant C\left(\mathcal{R}+\left|\partial_{x} v_{n+1}^{1}\right|_{k-1, \mu, \gamma}+\left|\partial_{x}^{2} v_{n+1}^{2}\right|_{k-2, \mu, \gamma}+\left|\partial_{x}^{2} v_{n+1}^{1}\right|_{k-2, \mu, \gamma}\right) .
\end{aligned}
$$

This estimate seems to require a little more care.

Consider for example

$$
\frac{1}{\varepsilon}\left|\mathbb{A} \partial_{x}^{2} v_{n+1}^{1}\right|_{k-3, \mu, \gamma}
$$

which yields terms

$$
\frac{1}{\varepsilon} \mu^{k-3-j} C\left|\partial^{\langle s\rangle}\left(\varepsilon^{L-1} w_{n}\right) \partial^{t} \partial_{x}^{2} v_{n+1}^{1}\right|_{0, \mu, \gamma},
$$

where $s+t \leqslant j \leqslant k-3$.

If $s=0,(6.103)$ is $\leqslant$

$$
\frac{C}{\varepsilon}\left|\partial_{x}^{2} v_{n+1}^{1}\right|_{k-3, \mu, \gamma} \leqslant \frac{C}{\gamma}\left|\partial_{x}^{2} v_{n+1}^{1}\right|_{k-2, \mu, \gamma} .
$$

If $s \neq 0$ (6.103) equals

$$
\begin{aligned}
& \mu^{k-3-j} C\left|\partial^{(s-1)} \partial\left(\varepsilon^{L-2} w_{n}\right) \partial^{t} \partial_{x}^{2} v_{n+1}^{1}\right|_{0, \mu, \gamma} \\
& \quad \leqslant C\left(\left|\partial\left(\varepsilon^{L-2} w_{n}\right)\right|_{*}\left|\partial_{x}^{2} v_{n+1}^{1}\right|_{k-4, \mu, \gamma}+\left|\partial\left(\varepsilon^{L-2} w_{n}\right)\right|_{k-4, \mu, \gamma}\left|\partial_{x}^{2} v_{n+1}^{1}\right|_{*}\right) .
\end{aligned}
$$

Now taking the derivative of (6.87)(a) we get

$$
\begin{aligned}
\left|\partial_{x}^{2} v_{n+1}^{1}\right|_{*} \leqslant & C\left(\frac{1}{\varepsilon}\left|\widetilde{D} w_{n+1}\right|_{*}+\left|\partial_{x} \widetilde{D} w_{n+1}\right|_{*}\right. \\
& \left.+\frac{1}{\varepsilon^{2}}\left|w_{n+1}\right|_{*}+\frac{1}{\varepsilon}\left|\partial_{x} w_{n+1}\right|_{*}+\varepsilon^{M-L-1}\right) \\
\leqslant & C\left(\frac{1}{\varepsilon^{2}}\left\|v_{n+1}, \phi_{n+1}\right\|^{* *}+\varepsilon^{M-L-1}\right) .
\end{aligned}
$$

Since

$$
\left|\partial\left(\varepsilon^{L-2} w_{n}\right)\right|_{k-4, \mu, \gamma} \leqslant \frac{\varepsilon^{3}}{\gamma^{3}}\left|\varepsilon^{L-2} w_{n}\right|_{k, \mu, \gamma},
$$

we obtain also from (6.105) an estimate compatible with (6.101).

The other terms in the expression for $\partial_{x}^{3} v_{n+1}^{2}$ are similar but easier to handle, so this concludes the proof of Proposition 6.13.

\subsubsection{Contraction}

NOTATION 6.17. - (1) Recall $w_{n}=\left(v_{n}, \sqrt{\varepsilon} \partial_{x, y} v_{n}^{2}, \phi_{n}, d \phi_{n}\right)$ and set $\zeta_{n+1}=w_{n+1}-w_{n}$.

(2) As before let $U^{a, \varepsilon}=\left(u^{a}, d \psi^{a}, \partial_{y}\left(u^{a}, d \psi^{a}\right), \varepsilon \partial_{x} u^{a}, \sqrt{\varepsilon}\right)$ and let $\mathbb{A}_{n}$ denote a smooth function

$$
\mathbb{A}_{n}=\mathbb{A}_{n}\left(U^{a, \varepsilon}, \varepsilon^{L-1} w_{n}\right)
$$

ANNALES SCIENTIFIQUES DE L'ÉCOLE NORMALE SUPÉRIEURE 
Denote by $\mathbb{B}_{n}$ a smooth function

$$
\mathbb{B}_{n}=\mathbb{B}_{n}\left(U^{a, \varepsilon}, \varepsilon \partial_{s}^{2} u^{a}, \partial_{x} u^{a}, \varepsilon^{L-1} w_{n}\right),
$$

where the second and third arguments only occur linearly in terms where they appear.

(3) Let $\mathbb{D}_{n}$ and $\mathbb{E}_{n}$ be smooth functions

$$
\begin{aligned}
& \mathbb{D}_{n}=\mathbb{D}_{n}\left(U^{a, \varepsilon}, \varepsilon^{L-1}\left(w_{n}, w_{n-1}\right)\right), \\
& \mathbb{E}_{n}=\mathbb{E}_{n}\left(U^{a, \varepsilon}, \varepsilon \partial_{s}^{2} u^{a}, \partial_{x} u^{a}, \varepsilon^{L-1}\left(w_{n}, w_{n-1}\right)\right),
\end{aligned}
$$

where the second and third arguments of $\mathbb{E}_{n}$ only occur linearly in terms where they appear.

(4) Let

$$
\Gamma(t, y) w_{n+1}=\left(\left[v_{n+1}\right],\left[\partial_{x} v_{n+1}^{2}\right], \partial_{t} \phi_{n+1}-\varepsilon \triangle_{y} \phi_{n+1}+\ell(t, y) \cdot v_{n+1}\right) .
$$

The transmission problem satisfied by $w_{n+1},(6.16)$, can be written

$$
\begin{gathered}
\mathbb{P}_{n} w_{n+1}:=\mathbb{A}_{n} D w_{n+1}+\mathbb{B}_{n} w_{n+1}=-\varepsilon^{M-L} \theta(t) R^{M}, \\
\Gamma w_{n+1}=0 \quad \text { on } x=0, \\
w_{n+1}=0 \quad \text { in } t<-T_{0} / 3 .
\end{gathered}
$$

Thus, the problem satisfied by $\zeta_{n+1}=w_{n+1}-w_{n}$ is

$$
\begin{gathered}
\mathbb{P}_{n} \zeta_{n+1}=-\left(\mathbb{P}_{n}-\mathbb{P}_{n-1}\right) w_{n}, \\
\Gamma \zeta_{n+1}=0 \quad \text { on } x=0, \\
\zeta_{n+1}=0 \quad \text { in } t<-T_{0} / 3 .
\end{gathered}
$$

The interior equation is

$$
\begin{aligned}
\mathbb{P}_{n} \zeta_{n+1} & =-\left(\mathbb{A}_{n}-\mathbb{A}_{n-1}\right) D w_{n}-\left(\mathbb{B}_{n}-\mathbb{B}_{n-1}\right) w_{n} \\
& =\varepsilon^{L-1} \zeta_{n} \mathbb{D}_{n} D w_{n}+\varepsilon^{L-1} \zeta_{n} \mathbb{E}_{n} w_{n}:=\mathbb{F}_{n} .
\end{aligned}
$$

The iterates satisfy the uniform estimates (6.65) for a $k$ such that $\frac{d}{2}+4<k<M-L$. Let

$$
\left.\left\|\zeta_{n}\right\||=| \zeta_{n}\right|_{0, \mu, \gamma}+\left|v_{n}(0)-v_{n-1}(0)\right|_{0, \mu, \gamma} .
$$

The $L^{2}$ estimate gives

$$
\left\|\zeta_{n+1}\right\| \leqslant \frac{C}{\sqrt{\gamma}}\left|\mathbb{F}_{n}\right|_{0, \mu, \gamma} \leqslant \frac{C}{\sqrt{\gamma}} \varepsilon^{L-2}\left|\zeta_{n}\right|_{0, \mu, \gamma} \leqslant \frac{C}{\sqrt{\gamma}} \varepsilon^{L-2}\left\|\zeta_{n}\right\|,
$$

where $L \geqslant 2$. For $\gamma$ fixed large enough and $0<\varepsilon \leqslant \varepsilon_{0}(\gamma)$ small enough, the iterates converge in the $\|w\|$ norm to some $\left(v, \sqrt{\varepsilon} \partial_{x, y} v^{2}, \phi, d \phi\right)$. In view of the estimates (6.65), a standard argument using weak convergence and interpolation shows that $(v, \phi)$ solves the nonlinear error problem (6.10) and also satisfies the estimates (6.65).

We summarize the results of this section and the paper in the following theorem:

THEOREM 6.18. -

(1) Assume (H0)-(H9) and (H10)(a). Let $\left(U^{0}, \psi^{0}\right)$ be an inviscid shock and $\left(u^{a}, \psi^{a}\right)$ an approximate solution of order $M$ as described in Proposition 5.7. Suppose $L \geqslant 2$ and that the constants $k, M$ satisfy

$$
\frac{d}{2}+4<k<M-L
$$

$4^{e}$ SÉRIE - TOME $39-2006-\mathrm{N}^{\circ} 1$ 
Suppose $s_{0}$, the index measuring Sobolev regularity of the shock, satisfies

$$
s_{0}>k+\frac{7}{2}+2 M+\frac{d+1}{2} .
$$

Fix $K>0$ arbitrarily large. For $\gamma$ fixed large enough and $\varepsilon_{0}(\gamma)$ small enough, the nonlinear forward error problem (6.10) has a unique solution $(v, \phi)$ for $0<\varepsilon \leqslant \varepsilon_{0}(\gamma)$ satisfying the estimates

$$
\begin{aligned}
& \text { (a) }\|v, \phi\|^{* * *} \leqslant 1 \\
& \text { (b) }\|v, \phi\|_{k, \mu, \gamma} \leqslant 2 C(\gamma) \varepsilon^{M-L-k}
\end{aligned}
$$

with norms as in Definition 6.10.

(2) If we take

$$
u=u^{a}+\varepsilon^{L} v, \quad \psi=\psi^{a}+\varepsilon^{L} \phi,
$$

then $(u, \psi)$ is an exact solution of the nonlinear transmission problem (6.1) on $\left[0, T_{0}\right] \times$ $\mathbb{R}_{y}^{d-1} \times\{|x| \leqslant K\}$.

Remark 6.19. - (1) Recalling the form of $\left(u^{a}, \psi^{a}\right)(5.44)-(5.45)$ and using the estimates (6.119), we derive Theorem 1.1 of the Introduction as an immediate corollary.

(2) It may appear that because of the use of the cutoff $\theta(t)$ in the forward error problem (6.10), we have only proved convergence of parabolic solutions to the inviscid shock on a time interval that is strictly shorter than the given time of existence of the inviscid shock. However, a uniformly stable shock on a given time interval $\left[-T_{0}, T_{0}\right]$ can always be extended to a strictly larger time interval by $[26,27]$. If the given inviscid shock is so extended, then our result gives convergence of parabolic solutions to the shock on the original time interval $\left[-T_{0}, T_{0}\right]$. Our result does not give convergence near the boundary points of the maximal time interval of existence of the inviscid shock.

\section{Appendix A}

\section{A.1. Low frequency expansion of the Evans function}

In this section we derive a low frequency expansion of the Evans function $D(q, \zeta)(2.53)$, which implies Proposition 2.23 as an immediate corollary. Earlier versions and proofs of the result were given in [36,37,39].

We give an expansion that is uniformly valid for $\hat{\zeta} \in \bar{S}_{+}^{d}$ and $q=\left(p, u^{\prime}, h^{\prime}\right)$ in a neighborhood $\omega$ of $\underline{q}=(\underline{p}, 0,0)$, where $\underline{p}=\left(\underline{u}^{+}, \underline{u}^{-}, \underline{h}\right) \in \mathcal{C}$. To describe the expansion we need some preparation.

\section{A.1.1. The Majda uniform stability determinant}

The partially linearized, transformed, inviscid shock problem associated to $\underline{q}$ considered by Majda [26] takes the form

$$
\begin{gathered}
\partial_{z} v-H_{0}(\underline{q}, \zeta) v=g, \\
\psi\left(\lambda\left[f_{0}\right]+\sum_{j=1}^{d-1} i \eta_{j}\left[f_{j}\right]\right)-\left[\widetilde{A}_{d} v\right]=h \quad \text { on } z=0,
\end{gathered}
$$

where $\lambda=(i \tau+\gamma), H_{0}^{ \pm}$is given by (3.183), $\left[f_{j}\right]:=f_{j}\left(u^{+}\right)-f_{j}\left(u^{-}\right)$, and $\widetilde{A}_{d}$ is evaluated at $( \pm \infty, q)$. The transmission condition in (A.1) is the (full) linearization of the Rankine-Hugoniot condition. 
For $q \in \omega$ and $\hat{\zeta} \in S_{+}^{d}=\{\hat{\zeta}=(\hat{\tau}, \hat{\gamma}, \hat{\eta}):|\hat{\zeta}|=1, \hat{\gamma}>0\}$ let $F_{H_{0}^{ \pm}}(q, \hat{\zeta})$ denote, respectively, the generalized eigenspace of $H_{0}^{ \pm}(q, \hat{\zeta})$ associated to eigenvalues $\mu$ with $\mp \Re \mu>0$. By hyperbolicity (H4) these spaces have dimensions independent of $\hat{\zeta}$ with $\hat{\gamma}>0$, and are $C^{\infty}$ on $\omega \times S_{+}^{d}$. By an argument of [19] (or [4, Chapter 7, Theorem 3.5]) combined with the block structure result of [29], they extend continuously to $\omega \times \bar{S}_{+}^{d}$. Set

$$
m(\underline{q}, \hat{\zeta})=\hat{\lambda}\left[f_{0}\right]+\sum_{j=1}^{d-1} i \hat{\eta}_{j}\left[f_{j}\right] .
$$

By the proof of Corollary 3.45, $F_{H_{0}^{ \pm}}(\underline{q}, \hat{\zeta})$ have dimensions $N-N_{ \pm}$respectively, for a total dimension of $N-1$. We write $\widetilde{A}_{d}^{ \pm} F_{H_{0}^{ \pm}}(\underline{q}, \hat{\zeta})$ for the images of the spaces under $\widetilde{A}_{d}( \pm \infty, \underline{q})$.

Definition A.1. - $[26,27]$ The Majda determinant associated to $\underline{q}$ is the $N \times N$ determinant

$$
\triangle(\underline{q}, \hat{\zeta}):=\operatorname{det}\left(\widetilde{A}_{d}^{-} F_{H_{0}^{-}}(\underline{q}, \hat{\zeta}), \widetilde{A}_{d}^{+} F_{H_{0}^{+}}(\underline{q}, \hat{\zeta}), m(\underline{q}, \hat{\zeta})\right) .
$$

The shock $\underline{p}$ is uniformly stable in the sense of Majda if $\triangle(\underline{q}, \hat{\zeta}) \neq 0$ for $\hat{\zeta} \in \bar{S}_{+}^{d}$.

Majda showed, for example, that when the planar shock $p$ is uniformly stable, there exist nearby, piecewise $C^{1}$, curved inviscid shocks for a finite time.

\section{A.1.2. Main result}

The following theorem immediately implies Proposition 2.23. Recall

$$
D(q, \zeta)=\operatorname{det}\left(\mathbb{E}^{ \pm}(q, \zeta), \mathbb{E}^{-}(q, \zeta)\right)
$$

and set $D(q, \hat{\zeta}, \rho):=D(q, \rho \hat{\zeta})$ for $|\zeta|>0$.

Theorem A.2. - Assume (H0)-(H2) and (H4)-(H8). There exists a neighborhood $\omega$ of $\underline{q}$, $\rho_{0}>0$, and functions $c(q, \hat{\zeta}, \rho), D_{m}(q, \hat{\zeta}, \rho)$ both continuous on $\omega \times \bar{S}_{+}^{d} \times\left[0, \rho_{0}\right]$ and $C^{\infty}$ in $\rho>0$ such that

$$
D(q, \hat{\zeta}, \rho)=\rho c(q, \hat{\zeta}, \rho) D_{m}(q, \hat{\zeta}, \rho)
$$

Here $c(q, \hat{\zeta}, \rho)$ is nonvanishing on $\omega \times \bar{S}_{+}^{d} \times\left[0, \rho_{0}\right]$ and

$$
D_{m}(\underline{q}, \hat{\zeta}, 0)=\beta(\underline{q}) \triangle(\underline{q}, \hat{\zeta}),
$$

where $\triangle$ is the Majda determinant, and $\beta(q)$ is $C^{\infty}$ on $\omega$ and nonvanishing at $q$ if and only if the connection $W(z, \underline{q})$ is transversal in the sense of Definition 2.12 .

\section{A.1.3. Slow modes and fast modes}

To compute $D(q, \zeta)$ for $|\zeta|$ small, we make a special choice of basis. For $\zeta \in \overline{\mathbb{R}}^{d+1} \backslash 0$ let

$$
F^{ \pm}(q, \zeta)=F_{H_{ \pm}}(q, \zeta) \oplus F_{P_{ \pm}}(q, \zeta),
$$

where $F_{H_{ \pm}}$are the generalized eigenspaces of $H_{ \pm}$(3.182) associated to eigenvalues $\mu$ with $\mp \Re \mu>0$, and $F_{P_{ \pm}}$are defined similarly. The properties of the conjugators $\mathcal{W}_{ \pm}(z, q, \zeta)(3.171)$, $\mathcal{V}_{ \pm}(q, \zeta)(3.182)$ imply

$$
\mathbb{E}^{ \pm}(q, \zeta)=\mathcal{W}_{ \pm}(0, q, \zeta) \mathcal{V}_{ \pm}(q, \zeta) F^{ \pm}(q, \zeta)
$$

The spaces $F_{P_{ \pm}}(q, \zeta)$ are $C^{\infty}$ for $q \in \omega$ and $|\zeta| \leqslant \rho_{0}$ small. By the analysis of [31] the spaces $F_{H_{ \pm}}(q, \hat{\zeta}, \rho):=F_{H_{ \pm}}(q, \rho \hat{\zeta})$ are $C^{\infty}$ for $\rho \in\left(0, \rho_{0}\right]$ and extend continuously to $\left[0, \rho_{0}\right]$. Since 


$$
H_{ \pm}(q, \hat{\zeta}, \rho)=\rho H_{0}^{ \pm}(q, \hat{\zeta})+\mathrm{O}\left(\rho^{2}\right)
$$

we have in particular

$$
F_{H_{ \pm}}(q, \hat{\zeta}, 0)=F_{H_{0}^{ \pm}}(q, \hat{\zeta})
$$

We now choose bases $\left\{s_{ \pm}^{j}(q, \hat{\zeta}, \rho)\right\}_{j=1, \ldots, N-N_{ \pm}}$for $F_{H_{ \pm}}(q, \hat{\zeta}, \rho)$ with the same regularity as the spaces, and bases $\left\{c_{ \pm}^{j}\right\}_{j=1, \ldots, s_{ \pm}}$for $F_{P_{ \pm}}(\underline{q}, 0)$.

Remark A.3. - Here as in Remark 3.31, after taking $\omega$ to be a small ball, for example, we obtain global bases $\left\{s_{ \pm}^{j}(q, \hat{\zeta}, \rho)\right\}_{j=1, \ldots, N-N_{ \pm}}$with the stated regularity by applying the fact that contractible base spaces admit only trivial bundles [35, Corollary 11.6]. Indeed,

$$
\omega \times \bar{S}_{+}^{d} \times\left[0, \rho_{0}\right]
$$

is contractible.

Next, in $\pm z \geqslant 0$ we define $N-N_{ \pm}$slow modes

$$
\begin{aligned}
& U_{ \pm}^{j}(z, q, \hat{\zeta}, \rho):=\mathcal{W}_{ \pm}(z, q, \zeta) \mathcal{V}_{ \pm}(q, \zeta)\left(\begin{array}{c}
e^{z H_{ \pm}(q, \hat{\zeta}, \rho)} s_{ \pm}^{j}(q, \hat{\zeta}, \rho) \\
0
\end{array}\right) \\
& \quad j=1, \ldots, N-N_{ \pm},
\end{aligned}
$$

and $s_{ \pm}$fast modes

$$
\begin{aligned}
& \mathbb{U}_{ \pm}^{j}(z, q, \zeta)=\mathcal{W}_{ \pm}(z, q, \zeta) \mathcal{V}_{ \pm}(q, \zeta)\left(\begin{array}{c}
0 \\
e^{z P_{ \pm}(q, \zeta)} \pi_{ \pm}(q, \zeta) c_{ \pm}^{j}
\end{array}\right) \\
& \quad j=1, \ldots, s_{ \pm},
\end{aligned}
$$

where $\pi_{ \pm}(q, \zeta)$ are projections onto the $\mp$ generalized eigenspaces of $P_{ \pm}(q, \zeta)$. In addition we may choose $c_{ \pm}^{s_{ \pm}}$so that

$$
\mathbb{U}_{ \pm}^{s_{ \pm}}(z, \underline{q}, 0)=\left(\begin{array}{c}
\partial_{z} W(z, \underline{q}) \\
\partial_{z}^{2} W_{2}(z, \underline{q})
\end{array}\right) \quad \text { in } \pm z \geqslant 0
$$

where $W(z, \underline{q})$ is the shock profile associated to $\underline{p}$.

Since $\mathcal{W}_{ \pm} \rightarrow I$ as $z \rightarrow \pm \infty$ and $\mathcal{V}_{ \pm}(q, 0)$ has the structure (3.184), we obtain immediately from (A.12), (A.13):

$$
\begin{aligned}
& \lim _{z \rightarrow \pm \infty} U_{ \pm}^{j}(z, q, \hat{\zeta}, 0)=\left(\begin{array}{c}
s_{ \pm}^{j}(q, \hat{\zeta}, 0) \\
0
\end{array}\right), \quad j=1, \ldots, N-N_{ \pm}, \\
& \lim _{z \rightarrow \pm \infty} \mathbb{U}_{ \pm}^{j}(z, q, 0)=\left(\begin{array}{l}
0 \\
0
\end{array}\right), \quad j=1, \ldots, s_{ \pm} .
\end{aligned}
$$

Proof of Theorem A.2. - 1. We may write

$$
\begin{gathered}
D(q, \hat{\zeta}, \rho)=c(q, \hat{\zeta}, \rho) \operatorname{det}\left(\left\{U_{-}^{j}\right\}_{1 \leqslant j \leqslant N-N_{-}},\left\{\mathbb{U}_{-}^{j}\right\}_{1 \leqslant j \leqslant s_{-}},\right. \\
\left.\left\{U_{+}^{j}\right\}_{1 \leqslant j \leqslant N-N_{+}},\left\{\mathbb{U}_{+}^{j}\right\}_{1 \leqslant j \leqslant s_{+}}\right)\left.\right|_{z=0} \\
:=c(q, \hat{\zeta}, \rho) D_{1}(q, \hat{\zeta}, \rho),
\end{gathered}
$$

for a function $c(q, \hat{\zeta}, \rho)$ as described in the theorem.

2. For $\mathcal{L}\left(z, q, \zeta, \partial_{z}\right)$ as in (3.14) we first rewrite the linearized problem $\mathcal{L} u=0$ on $\pm z \geqslant 0$ in a form that will allow us to use the conservative structure to simplify the determinant. For $q=(p, 0,0)$ and with $\widetilde{A}_{j}, \widetilde{B}_{j, k}$ as in (3.14), (3.15) set 


$$
\mathcal{A}_{j}(W(z, q), \nu(h)) u:=\widetilde{A}_{j}(z, q) u-\left(u \cdot d_{u} \widetilde{B}_{j, d}\right) W_{z}, \quad j=1, \ldots, d .
$$

Then $\mathcal{L}\left(z, q, \zeta, \partial_{z}\right) u=0$ can be rewritten:

$$
\begin{aligned}
0= & -\left(\widetilde{B}_{d, d} u_{z}\right)_{z}+\lambda A_{0} u+\sum_{j=1}^{d-1} i \eta_{j} \mathcal{A}_{j} u+\left(\mathcal{A}_{d} u\right)_{z} \\
& -\sum_{j=1}^{d-1} i \eta_{j} \widetilde{B}_{j, d} u_{z}-\sum_{k=1}^{d-1} i \eta_{k}\left(\widetilde{B}_{d, k} u\right)_{z}+\sum_{j, k=1}^{d-1} \widetilde{B}_{j, k} \eta_{j} \eta_{k} u .
\end{aligned}
$$

3. Taking $\zeta=0$ in (A.18) and integrating $\int_{ \pm \infty}^{z}$ in $\pm z \geqslant 0$, we find

$$
-\widetilde{B}_{d, d} u_{z}+\mathcal{A}_{d} u \text { is constant on } \pm z \geqslant 0 .
$$

By (A.15) this constant is 0 for fast modes and $\widetilde{A}_{d}( \pm \infty, q) s_{ \pm}^{j}(q, \hat{\zeta}, 0)$ for slow modes. Taking components and rearranging slightly in (A.19), we find that the $j$ th fast or slow mode satisfies, on $\pm z \geqslant 0$ :

$$
\text { (fast) } \begin{aligned}
\widetilde{A}_{d}^{11} u_{1}+\widetilde{A}_{d}^{11} u_{2} & =0, \\
-\widetilde{B}_{d, d}^{22} u_{2 z}+\mathcal{A}_{r} u_{2} & =0, \quad \text { where } \mathcal{A}_{r}=\mathcal{A}_{d}^{22}-\mathcal{A}_{d}^{21}\left(\widetilde{A}_{d}^{11}\right)^{-1} \widetilde{A}_{d}^{12}
\end{aligned}
$$

and

$$
\text { (slow) } \begin{aligned}
\widetilde{A}_{d}^{11} u_{1}+\widetilde{A}_{d}^{11} u_{2} & =\left(\widetilde{A}_{d}( \pm \infty, q) s_{ \pm}^{j}(q, \hat{\zeta}, 0)\right)_{1}, \\
-\widetilde{B}_{d, d}^{22} u_{2 z}+\mathcal{A}_{r} u_{2} & =\left(\widetilde{A}_{d}( \pm \infty, q) s_{ \pm}^{j}(q, \hat{\zeta}, 0)\right)_{2} .
\end{aligned}
$$

4. The matrix coefficients on the left in (A.20), (A.21) are continuous across $z=0$, so we can use these row operations to simplify the first and third rows of the determinant in (A.16). Let the slow (respectively fast) columns of the new determinant so obtained be denoted by $V_{ \pm}^{j}$ (respectively $\mathbb{V}_{ \pm}^{j}$ ), where $\mathbb{V}_{ \pm}^{j}=\left(\mathrm{u}_{ \pm 1}^{j}, \mathrm{u}_{ \pm 2}^{j}, \mathrm{u}_{ \pm 3}^{j}\right)$. (A.20) implies that $\rho$ can be smoothly factored out of the entries of the fast $N \times(s+1)$ submatrix,

$$
\left(\left(\begin{array}{c}
u_{-1}^{j} \\
u_{-3}^{j}
\end{array}\right)_{1 \leqslant j \leqslant s_{-}}\left(\begin{array}{c}
u_{+1}^{j} \\
u_{+3}^{j}
\end{array}\right)_{1 \leqslant j \leqslant s_{+}}\right) .
$$

Since every term in the expanded full determinant contains at least one factor given by such an entry from (A.22), this implies (A.5) with $D_{m}$ continuous up to $\rho=0$.

5. To see how the jump column in $D_{m}(q, \hat{\zeta}, 0)$ arises, we examine the variation in $\mathbb{U}_{ \pm}^{s_{ \pm}}$with respect to $\rho$ near $\rho=0$. Suppose $u=u^{+}$in (A.18) gives the first two components of the fast mode $\mathbb{U}_{+}^{s_{+}}$, and on $z \geqslant 0$ set

$$
p^{+}(z, \underline{q}, \hat{\zeta}):=\partial_{\rho} u^{+}(z, \underline{q}, \hat{\zeta}, 0) .
$$

In (A.18) write $(\lambda, \eta)=\rho(\hat{\lambda}, \hat{\eta})$ and apply $\left.\partial_{\rho}\right|_{\rho=0}$ to get

$$
\begin{aligned}
0= & -\left(\widetilde{B}_{d, d} p_{z}^{+}\right)_{z}+\left(\mathcal{A}_{d} p^{+}\right)_{z}+\hat{\lambda} A_{0} u^{+}+\sum_{j=1}^{d-1} i \hat{\eta}_{j} \mathcal{A}_{j} u^{+} \\
& -\sum_{j=1}^{d-1} i \hat{\eta}_{j} \widetilde{B}_{j, d} u_{z}^{+}-\sum_{k=1}^{d-1} i \hat{\eta}_{k}\left(\widetilde{B}_{d, k} u^{+}\right)_{z} \cdot
\end{aligned}
$$

$4^{e}$ SÉRIE - TOME $39-2006-\mathrm{N}^{\circ} 1$ 
Now recall (A.14) and the definition of $\mathcal{A}_{j}$ (A.17) to see that the right side of (A.24) is a perfect derivative. Integrate $\int_{+\infty}^{z}$ on $z \geqslant 0$ to find

$$
\begin{aligned}
0= & -\widetilde{B}_{d, d} p_{z}^{+}+\mathcal{A}_{d} p^{+}+\hat{\lambda}\left(f_{0}(W(z, \underline{q}))-f_{0}\left(u^{+}\right)\right) \\
& +\sum_{j=1}^{d-1} i \hat{\eta}_{j}\left(f_{j}(W(z, \underline{q}))-f_{j}\left(u^{+}\right)-\widetilde{B}_{j, d} W_{z}\right)-\sum_{k=1}^{d-1} i \hat{\eta}_{k} \widetilde{B}_{d, k} W_{z} .
\end{aligned}
$$

With $u^{-}$being the first two components of $\mathbb{U}_{-}^{s_{-}}$, we can obtain a similar equation for $p^{-}=$ $\left.\partial_{\rho}\right|_{\rho=0} u^{-}$. Setting $\mathbf{p}:=p^{+}-p$ and subtracting the equation for $p^{-}$from (A.25) at $z=0$ we obtain

$$
-\widetilde{B}_{d, d} \mathbf{p}_{z}+\mathcal{A}_{d} \mathbf{p}=\hat{\lambda}\left[f_{0}\right]+\sum_{j=1}^{d-1} i \hat{\eta}_{j}\left[f_{j}\right]=m(\underline{q}, \hat{\zeta})
$$

or in components

$$
\begin{aligned}
\widetilde{A}_{d}^{11} \mathbf{p}_{1}+\widetilde{A}_{d}^{11} \mathbf{p}_{2} & =m_{1}(\underline{q}, \hat{\zeta}), \\
-\widetilde{B}_{d, d}^{22} \mathbf{p}_{2 z}+\mathcal{A}_{r} \mathbf{p}_{2} & =m_{2}(\underline{q}, \hat{\zeta}) .
\end{aligned}
$$

6. We proceed finally to compute the determinant $D_{1}(\underline{q}, \hat{\zeta}, 0)$ for $D_{1}$ as in (A.16). Subtract the $\mathbb{U}_{-}^{s_{-}}$column from the $\mathbb{U}_{+}^{s_{+}}$column, recall (A.14), and apply the row operations using (A.20), (A.21), and (A.27), to obtain

$$
\begin{aligned}
& D_{1}(\underline{q}, \hat{\zeta}, \rho) \\
& =\operatorname{det}\left(\widetilde{A}_{d}^{11}\right)^{-1} \operatorname{det}\left(-\widetilde{B}_{d, d}^{22}\right)^{-1}
\end{aligned}
$$

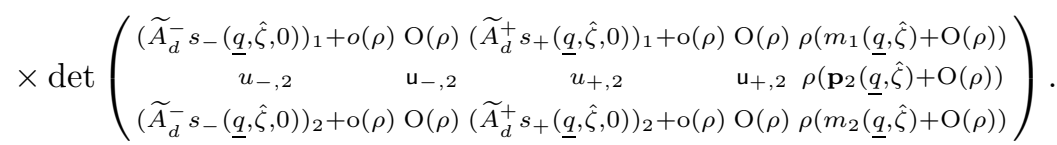

Here, for example, the five submatrices in the second row of the large matrix each contain $s$ rows and, respectively, $N-N_{-}, s_{-}, N-N_{+}, s_{+}-1$, and 1 column(s). Factoring $\rho$ out of the last column, we obtain (A.5) at $(\underline{q}, \hat{\zeta}, \rho)$ with $c(\underline{q}, \hat{\zeta}, \rho)$ the same as before. Recalling (A.10) and computing $D_{m}(\underline{q}, \hat{\zeta}, 0)$ using (A.28), we get (A.6) after a few switches of rows and columns, where

$$
\beta(q)=\operatorname{det}\left(\widetilde{A}_{d}^{11}\right)^{-1} \operatorname{det}\left(-\widetilde{B}_{d, d}^{22}\right)^{-1} \operatorname{det}\left(\mathbf{u}_{-, 2} \mathbf{u}_{+, 2}\right)
$$

up to a sign. The linearized equation (A.18) coincides with the linearized profile equation at $\rho=0$. Thus, recalling (A.14) we see that the last factor on the right in (A.29), an $s \times s$ determinant of fast modes, is nonvanishing at $\underline{q}$ exactly when the connection $W(z, \underline{q})$ is transversal.

\section{REFERENCES}

[1] Alinhac S., Existence d'ondes de raréfaction pour des sytèmes quasi-linéaires hyperboliques multidimensionnels, Comm. Partial Differential Equations 14 (1989) 173-230.

[2] Bony J.M., Calcul symbolique et propagation des singularités pour les équations aux dérivées partielles non linéaires, Ann. Sci. École Norm. Sup. Paris 14 (1981) 209-246.

[3] Coppel W.A., Stability and Asymptotic Behavior of Differential Equations, D.C. Heath, Boston, 1965. 
[4] Chazarain J., Piriou A., Introduction to the Theory of Linear Partial Differential Equations, NorthHolland, Amsterdam, 1982.

[5] Erpenbeck J.J., Stability of step shocks, Phys. Fluids 5 (1962) 1181-1187.

[6] FreistüHler H., Szmolyan P., Spectral stability of small shock waves, Arch. Rat. Mech. Anal. 164 (2002) 287-309.

[7] Gardner R., Zumbrun K., The gap lemma and geometric criteria instability of viscous shock profiles, CPAM 51 (1998) 797-855.

[8] Gilbarg D., The existence and limit behavior of the one-dimensional shock layer, Amer. J. Math. 73 (1951) 256-274.

[9] GuÈs O., Développements asymptotiques de solutions exactes de systèmes hyperboliques quasilinéaires, Asymp. Anal. 6 (1993) 241-270.

[10] GuÈs O., Métivier G., Williams M., Zumbrun K., Multidimensional viscous shocks I: degenerate symmetrizers and long time stability, J. Amer. Math. Soc. 18 (2005) 61-120.

[11] GuÈs O., MÉtivier G., Williams M., Zumbrun K., Multidimensional viscous shocks II: the small viscosity problem, Comm. Pure Appl. Math. 57 (2004) 141-218.

[12] Guès O., Métivier G., Williams M., Zumbrun K., Existence and stability of multidimensional shock fronts in the vanishing viscosity limit, Arch. Rat. Mech. Anal. 175 (2004) 151-244.

[13] GuÈs O., MÉTIVIER G., Williams M., ZuMBrun K., Stability of noncharacteristic boundary layers for the compressible Navier-Stokes and MHD equations, in preparation.

[14] GuÈs O., Métivier G., Williams M., Zumbrun K., Viscous boundary value problems for symmetric systems with variable multiplicities, in preparation.

[15] Goodman J., Nonlinear asymptotic stability of viscous shock profiles for conservation laws, Arch. Rat. Mech. Anal. 95 (1986) 325-344.

[16] Goodman J., XIN Z., Viscous limits for piecewise smooth solutions to systems of conservation laws, Arch. Rat. Mech. Anal. 121 (1992) 235-265.

[17] GuÈs O., Williams M., Curved shocks as viscous limits: a boundary problem approach, Indiana Univ. Math. J. 51 (2002) 421-450.

[18] Hörmander L., The Analysis of Linear Partial Differential Operators I, Springer, Berlin, 1983.

[19] Kreiss H.-O., Initial boundary value problems for hyperbolic systems, Comm. Pure Appl. Math. 23 (1970) 277-298.

[20] Kato T., Perturbation Theory for Linear Operators, Springer, Berlin, 1985.

[21] KawASHima S., Systems of hyperbolic-parabolic type with applications to the equations of magnetohydrodynamics, PhD thesis, Kyoto University, 1983.

[22] KaWAshima S., ShizUta Y., Systems of equations of hyperbolic-parabolic type with applications to the discrete Boltzmann equation, Hokkaido Math. J. 14 (1985) 249-275.

[23] KaWASHIMA S., SHIZUTA Y., On the normal form of the symmetric hyperbolic-parabolic systems associated with the conservation laws, Tohoku Math. J. (1988) 449-464.

[24] Mascia C., Zumbrun K., Pointwise Green function bounds for shock profiles with degenerate viscosity, Arch. Rat. Mech. Anal. 169 (2003) 177-263.

[25] Majda A., Pego R., Stable viscosity matrices for systems of conservation laws, J. Differential Equations 56 (1985) 229-262.

[26] Majda A., The Stability of Multidimensional Shock Fronts, Mem. Amer. Math. Soc., vol. 275, AMS, Providence, RI, 1983.

[27] Majda A., The Existence of Multidimensional Shock Fronts, Mem. Amer. Math. Soc., vol. 281, AMS, Providence, RI, 1983.

[28] MÉTIVIER G., Stability of Multidimensional Shocks, in: Advances in the Theory of Shock Waves, in: Progress in Nonlinear PDE, vol. 47, Birkhäuser, Boston, 2001.

[29] Métivier G., The block structure condition for symmetric hyperbolic systems, Bull. Lond. Math. Soc. 32 (2000) 689-702.

[30] Métivier G., ZuMbrun K., Large viscous boundary layers for noncharacteristic nonlinear hyperbolic problems, Mem. Amer. Math. Soc. 175 (826) (2005), vi+107 p.

[31] Métivier G., Zumbrun K., Symmetrizers and continuity of stable subspaces for parabolichyperbolic boundary value problems, Disc. Cont. Dyn. Syst. 11 (2004) 205-220.

$4^{\mathrm{e}}$ SÉRIE - TOME $39-2006-\mathrm{N}^{\circ} 1$ 
[32] Métivier G., Zumbrun K., Hyperbolic boundary value problems for symmetric systems with variable multiplicities, J. Differential Equations 211 (2005) 61-134.

[33] Pego R., Stable viscosities and shock profiles for systems of conservation laws, Trans. Amer. Math. Soc. 282 (1984) 749-763.

[34] Plaza R., Zumbrun K., An Evans function approach to spectral stability of small-amplitude shock profiles, J. Disc. Cont. Dyn. Syst. 10 (2004) 885-924.

[35] Steenrod N., The Topology of Fibre Bundles, Princeton University Press, Princeton, NJ, 1951.

[36] ZuMbrun K., Multidimensional stability of planar viscous shock waves, in: Advances in the Theory of Shock Waves, in: Progress in Nonlinear PDE, vol. 47, Birkhäuser, Boston, 2001, pp. 304-516.

[37] ZUMBRUN K., Stability of large-amplitude shock waves of compressible Navier-Stokes equations, with an appendix by H.K. Jenssen and G. Lyng, in: Handbook of Mathematical Fluid Dynamics, vol. 3, North-Holland, Amsterdam, 2004, pp. 311-533.

[38] ZuMbrun K., Planar stability criteria for viscous shock waves of systems with real viscosity, in: Hyperbolic Systems of Balance Laws, Springer Lecture Notes, 2006, in press.

[39] Zumbrun K., SERre D., Viscous and inviscid stability of multidimensional planar shock fronts, Indiana Univ. Math. J. 48 (1999) 937-992.

(Manuscrit reçu le 16 mars 2005 ; accepté, après révision, le 16 décembre 2005.)

C.M.I. Olivier GUÈs

Université de Provence,

39 , rue Joliot-Curie,

13453 Marseille cedex 13, France

E-mail: gues@cmi.univ-mrs.fr

Guy MÉTIVIER

MAB

Université de Bordeaux 1,

351, cours de la Libération,

33405 Talence cedex, France

E-mail: guy.metivier@math.u-bordeaux1.fr

Mark WiLLiams

Department of Mathematics, CB 3250,

University of North Carolina,

Chapel Hill, NC 27599, USA

E-mail: williams@email.unc.edu

Kevin ZUMBRUN

Indiana University,

Department of Mathematics, Rawles Hall,

Bloomington, IN 47405, USA

E-mail: kzumbrun@indiana.edu 